\title{
Rezente und subfossile Mikrobialithe westaustralischer Salzseen
}

\author{
Dissertation \\ zur Erlangung des Doktorgrades \\ der Mathematisch-Naturwissenschaftlichen Fakultäten \\ der Georg-August-Universität zu Göttingen
}

vorgelegt von

Meike Caselmann, geb. Pache

aus Braunschweig

Göttingen 2005 
D 7

Referent: Prof. Dr. Joachim Reitner

Korreferent: Prof. Dr. Volker Thiel

Tag der mündlichen Prüfung: 20.05.2005 


\section{Zusammenfassung}

Die Erforschung von Verkalkungsprozessen an organischen Matrizen und Biofilmen ist seit Jahren im Fokus karbonatsedimentologischer und biogeochemischer Untersuchungen. Cyanobakterien-reichen Biofilmen werden dabei Schlüsselfunktionen zugeschrieben. Die Bildungsmechanismen und der Umfang von cyanobakterieller Beteiligung an mineralisierenden und gefügebildenden Prozessen, besonders im fossilen Bericht, beruhen vielfach nur auf Vermutungen, sind oftmals umstritten und werden kontrovers diskutiert.

Untersuchungen an rezenten Mikrobialithen können zum besseren Verständnis der Bildungsprozesse und zur Rekonstruktion der Umweltbedingungen fossiler Mikrobialithe beitragen. So können eventuell Umweltbedingungen und Modelle über die an der Gesteinsbildung beteiligten Organismen innerhalb der verkalkenden Biofilme in den fossilen Bericht übertragen werden. Aktives Mikrobialith-Wachstum ist für wenige marine, aber für zahlreiche lakustrine Fallbeispiele belegt worden. Bekannte Vorkommen heutiger lakustriner Mikrobialithe sind unter anderem von den westaustralischen Salzseen beschrieben.

In der vorliegenden Arbeit wurden Stromatolith- und Thrombolith-Typen der westaustralischen Salzseen Lake Thetis und Lake Clifton sowie deren rezente Biofilme untersucht. Weitere westaustralische Seen und die marin-geprägte Shark Bay wurden zum Vergleich herangezogen.

Das hydrochemische Milieu aller fünf Salzseen und der Shark Bay wurde charakterisiert. Es zeigte sich, dass alle Seen eine Na-Cl-Dominanz aufweisen. Die evaporativ geprägten Seen entsprechen in den Ionenverhältnissen dem Meerwasser, enthalten jedoch weniger Kalzium. Die $\mathrm{Ca}^{2+}$-Konzentrationen in den Seen Lake Thetis und Lake Clifton hängen zum einen mit Wechselwirkungen mit sublakustrinem Grundwasser, zum anderen mit subfossiler wie auch rezenter Karbonatfällung zusammen.

Zur Charakterisierung der fossilen bis subfossilen Mikrobialithe wurde deren fossile Cyanobakterien-Vergesellschaftung und die geochemische Zusammensetzung $\left(\delta^{13} \mathrm{C}\right.$ und $\delta^{18} \mathrm{O}$, Haupt- und Spurenelemente, Kohlenstoff und Stickstoff, Biomarker) ermittelt.

Die mikrobiellen Karbonate des Lake Thetis enthalten neben mikrokristallinem, klumpigem Aragonit, terrigenen Komponenten und „Organomikrit“-Krusten sehr gut erhaltene Cyanobakterien (Phormidium sp., Scytonema sp., Entophysalis sp.). In jüngeren Stromatolithen des Lake Thetis sind Letztere in Form von Lamellen in Wechsellagerung überliefert. Stromatolith-Typen älterer Bioherme zeichnen sich durch geringere Anteile an Cyanobakterien und erhöhten terrigenen Eintrag aus. 
Eine amorphe Magnesium-Silizium-Phase ist in den subfossilen Stromatolithen des Lake Thetis in Form silifizierter Mikrofossilien (Scytonema-Scheiden) als unregelmäßige Lagen zwischen einschlussreichem Aragonit und feinlaminiertem „Organomikrit“ und als Rissfüllungen vertreten. Das Mg/Si-Molverhältnis der amorphen Phase beträgt $1: 1,1$. Rezente Biofilm-Karbonat-Oberflächen enthalten keine Mg-Si-Phase. Die Bildung der Mg-SiPhase in den fossilen Stromatolithen lässt sich eventuell auf geänderte Umweltbedingungen zurückführen.

Die subfossilen Thrombolithe des Lake Clifton wurden unter Beteiligung von Scytonema sp. gebildet. Rezent ist neben den Diatomeen vor allem Phormidium sp. in den Biofilmen dominierend. Eine amorphe $\mathrm{Mg}$-Si-Phase (Mg/Si-Molverhältnis von 1 : 1,66) stabilisiert die subfossilen Thrombolithe. Sie ist aus früheren Arbeiten nicht bekannt und spiegelt Entwicklungen des Sees wider (Zunahme der Eutrophierung und der Salinität, Stagnation des Thrombolith-Wachstums), die erst nach 1993 einsetzten.

Daten der stabilen C- und O-Isotope aus fossilen bis subfossilen Mikrobialith-Proben aller Seen ergeben keine für evaporative Systeme typische positive Kovarianz. Schwankungen innerhalb eines Lake Thetis-Stromatoliths mit ausgeprägter Wechsellagerung von „Entophysalis“- und „Scytonema-Phormidium“-Lamellen gehen nicht unmittelbar auf jahreszeitliche Schwankungen zurück. Die zum Teil negativen Kovarianzen gehen vermutlich auf eine Kombination von Evaporation, Überschichtung meteorischer Wässer und Schwankungen der Primärproduktion zurück.

Die Biomarker-Analysen der subfossilen Lake Thetis-Stromatolithe zeigen Diatomeen und Cyanobakterien als Primärproduzenten organischen Materials an. Das Schwefelpurpurbakterien-reiche Seebeckensediment des Lake Thetis liefert als Hauptkomponente Vaccensäure, welche ebenfalls in den subfossilen Stromatolithen des Seerands enthalten ist. Vaccensäure konnte erstmalig sowohl in einem durch MikrobialithWachstum dominierten lakustrinen Milieu als auch in subfossilen Stromatolithen nachgewiesen werden.

Diatomeen dominieren jahreszeitenunabhängig die Flora des Seewasserkörpers und die Oberflächen der Bioherme, sowohl im Lake Thetis als auch im Lake Clifton. Rezente Verkalkungsprozesse in Lake Thetis laufen während der Regenzeit an Scytonema-besiedelten Oberflächen mit Kontakt zum Seewasser ab. Während der Trockenzeit sind die Präzipitationsfronten an die Grenzbereiche der Gloeocapsa-EPS gekoppelt.

Gut definierte mikritische Cyanobakterien-Tuben, wie sie bei einer Photosynthese-gesteuerten Kalzifikation entstehen, kommen in den Seen nicht vor. Stattdessen finden im Lake Thetis 
Verkalkungen in Form mikrokristalliner, faseriger Aragonite an EPS-Randbereichen von Gloeocapsa-Kolonien, im Umfeld von Scytonema sp. und als zementierte Filamentspuren von Phormidium sp. statt.

Im Fall des Lake Clifton sind Scytonema sp. und Phormidium sp. in Form von Filamentspuren im mikrokristallinen Aragonit erkennbar und am Aufbau der subfossilen Thrombolithe beteiligt. Eine amorphe Mg-Si-Phase stabilisiert die Thrombolithe und wird als bisher letzte Bildungsphase interpretiert.

Um den Einfluss der Photosynthese auf eine Verschiebung des Karbonatgleichgewichtes abschätzen zu können, wurden Modellrechnungen (Programm PHREEQC) durchgeführt, welche einen photosynthetischen C-Entzug bei einem heutigen $\mathrm{CO}_{2}$-Partialdruck simulieren. Die Modellrechnungen zeigen, dass im Lake Clifton der Einfluss Photosynthese-gesteuerter $\mathrm{CaCO}_{3}$-Fällung im Seewasser vergleichsweise gering, in Mischwasserzonen allerdings höher ist. Das hier beobachtete Fehlen mikritischer Tuben geht auf die Pufferwirkung des Seewassers zurück, dessen zunehmende Eutrophierung vermutlich zu einer Dezimierung der Biofilmgemeinschaften (insbesondere Scytonema sp.) geführt hat. Im Fall des Lake Thetis gehen fehlende Cyanobakterien-Tuben vermutlich auf einen geringeren $\mathrm{Ca}^{2+}$-Eintrag und eine erhöhte pH-Pufferung zurück. Die Verkalkungen der Stromatolithe sind vermutlich auf Degradationsprozesse innerhalb der Biofilm-EPS zurückzuführen.

Als fossiles Analogon der Mikrobialithe des Lake Thetis und des Lake Clifton können die Grünalgen-Stromatolith-Bioherme des miozänen Riessees angesehen werden. Insbesondere die sogenannten „Sinterkrusten“ dieser miozänen Bioherme (Nördlinger Ries, Süddeutschland) gleichen den „Organomikrit“-Krusten des Lake Thetis. Zudem sind in jungen Ries-Ablagerungen Cyanobakterien gefunden worden, die morphologisch Scytonema sp. ähneln. Vom Riessee ist ein Wechsel vom „Soda“-See über Playa-Stadien hin zu einem Aussüßungsstadium bekannt. Lake Thetis wird als rezentes Gegenstück für den oberen Teil der im Profil „Hainsfarth“ aufgeschlossenen Grünalgen-Stromatolith-Bioherme angesehen. Entsprechend ist für dieses Stadium des Riessees eine hydrochemische Zusammensetzung anzunehmen, welche der des heutigen Lake Thetis ähnlich war. 


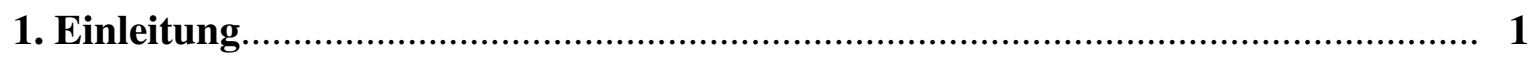

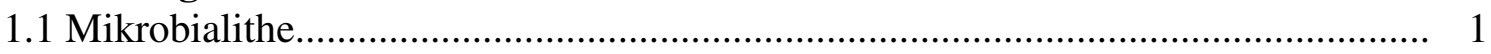

1.2 Faktoren der Verkalkungsprozesse................................................................. 4

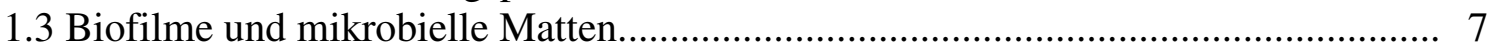

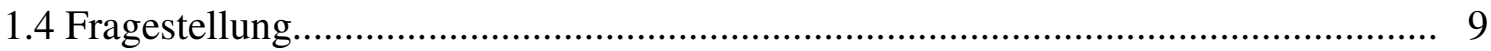

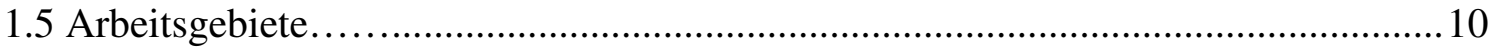

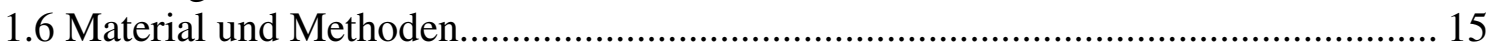

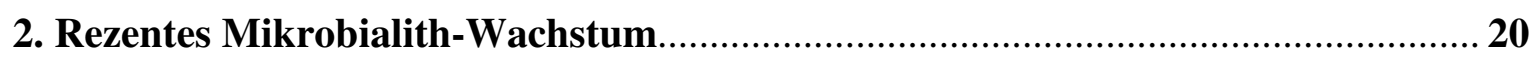

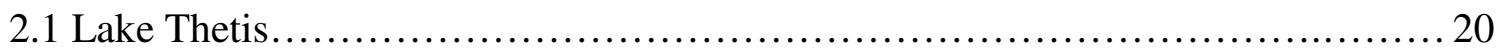

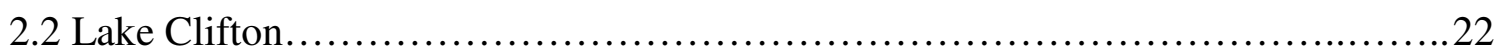

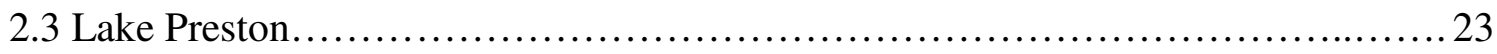

2.4 Lake Walyungup.......................................................... 24

2.5 Shark Bay................................................................. 24

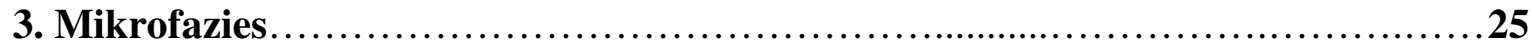

3.1 Mikrobialithe, Lake Thetis.............................................. 25

3.1.1 Beschreibung der Komponenten................................................................ 25

3.1.1.1 Biogene Komponenten................................................................... 25

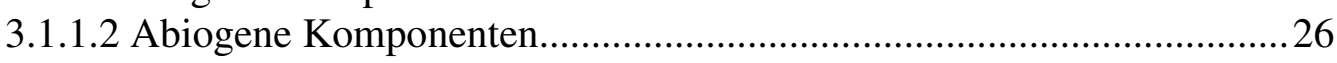

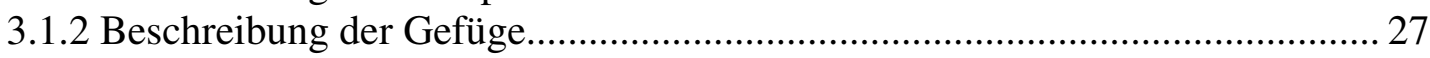

3.1.3 Beschreibung der Mikrobialith-Typen........................................................ 29

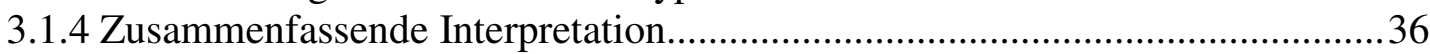

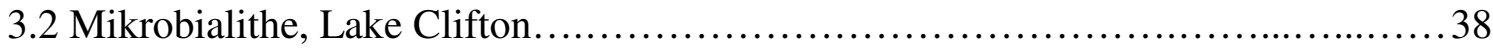

3.3 Mikrobialithe, Lake Pollard................................................ 39

3.4 Mikrobialithe, Lake Preston............................................... 39

3.5 Mikrobialithe, Lake Walyungup.......................................... 40

3.6 Mikrobialithe, Shark Bay................................................... 41

3.7 Vergleich von rezenten und fossilen Biofilmgemeinschaften..................................42

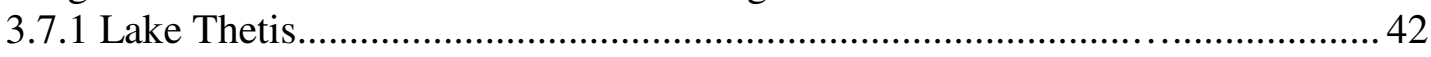

3.7.2 Lake Clifton.................................................... 42

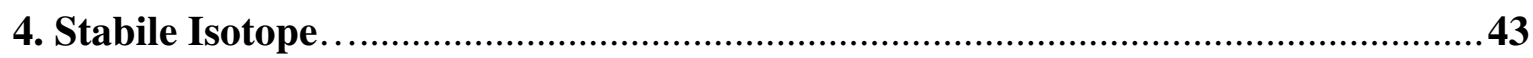

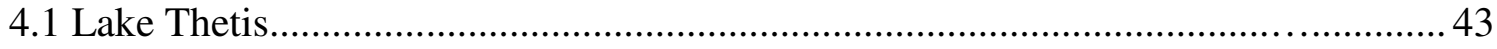

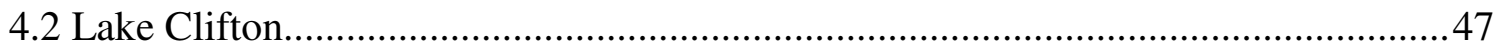

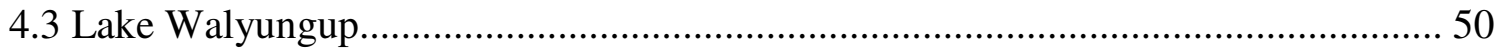

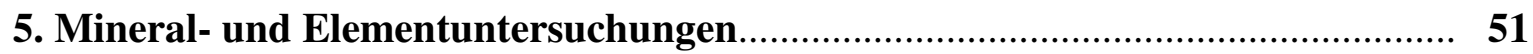

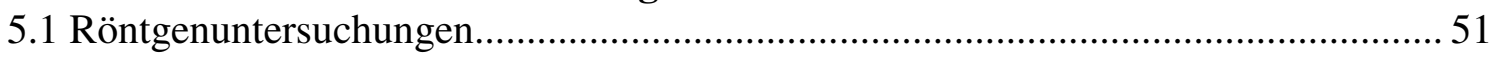

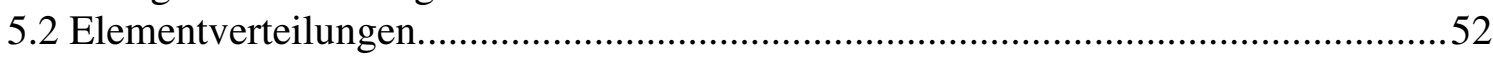

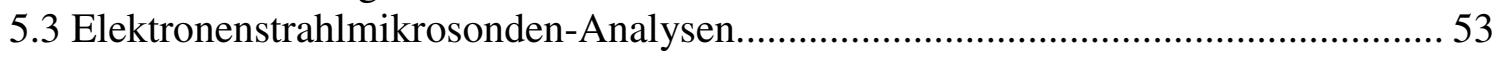

5.3.1 Subfossile Mikrobialithe, Lake Thetis................................ 53

5.3.2 Thrombolith, Lake Clifton............................................61

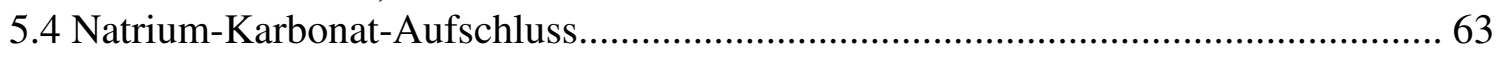




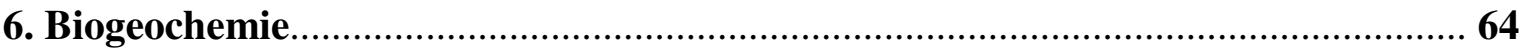

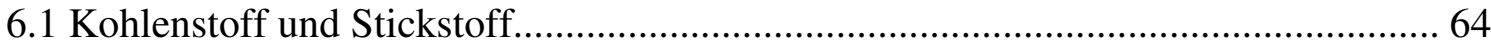

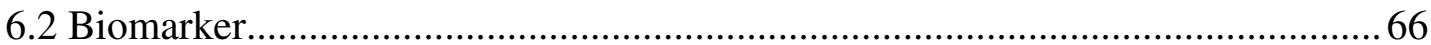

6.2.1 Subfossiler Stromatolith, Lake Thetis (LTh 98/14).........................................66 66

6.2.2 Subfossiler Stromatolith, „Organomikrit“-Lage, Lake Thetis (LTh 98/13).... 68

6.2.3 SPB-reiches Seebodensediment, Lake Thetis (LTh 02/20)............................68

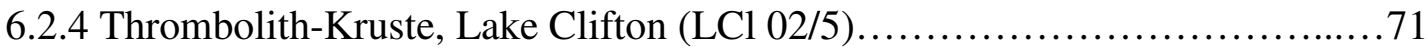

7. Hydrochemie

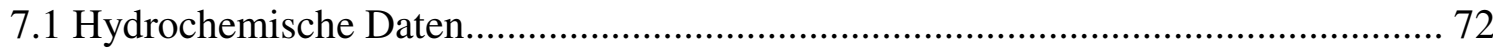

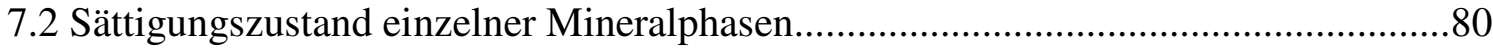

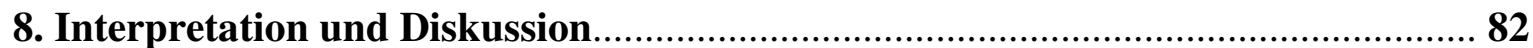

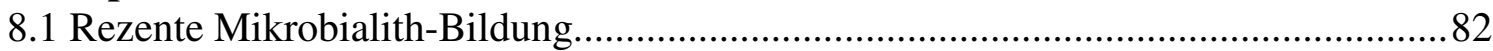

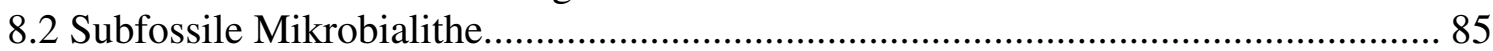

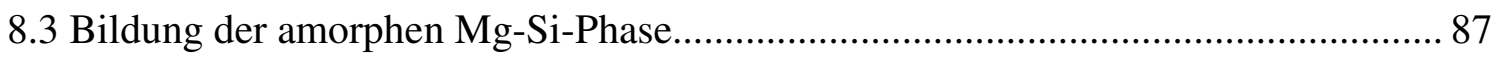

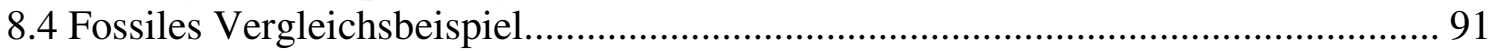

9. Resümee

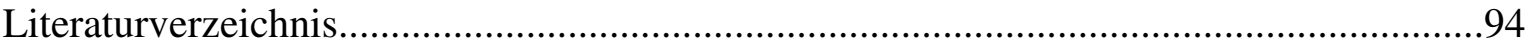

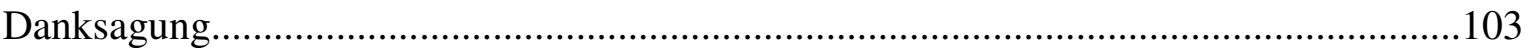

\section{Anhang}

Tafel 1: $\quad$ Subfossile Stromatolithe, Lake Thetis

Tafel 2: $\quad$ Subfossile Stromatolithe, Lake Thetis

Tafel 3: $\quad$ Rezente Biofilme, Lake Thetis

Tafel 4: $\quad$ Rezente Biofilme, Lake Thetis

Tafel 5: $\quad$ Rezente Biofilme, Lake Thetis

Tafel 6: $\quad$ Lake Thetis

Tafel 7: $\quad$ Lake Clifton

Tafel 8: $\quad$ Subfossile Thrombolithe und rezente Biofilme, Lake Clifton

Tafel 9: $\quad$ Subfossile Thrombolithe, Lake Clifton

Tafel 10: $\quad$ Lake Clifton

Tafel 11: $\quad$ Subfossile Mikrobialithe, Lake Preston und Lake Clifton

Tafel 12: $\quad$ Lake Walyungup

Tafel 13: $\quad$ Mikrobialithe, Lake Walyungup

Tafel 14: $\quad$ Shark Bay

Tafel 15: $\quad$ Rezente Biofilme, Shark Bay

Tafel 16: $\quad$ Stromatolithe, Shark Bay

Tafel 17: $\quad$ Mikrobialithe, Lake Preston und Lake Pollard

Tafel 18: $\quad$ Sedimentkerne, Spherulith-Proben

Anhang 1: $\quad$ Tabelle, Proben-Liste, Regenzeit 1998

Anhang 2: $\quad$ Tabelle, Proben-Liste, Trockenzeit 2002

Anhang 3: $\quad$ Abbildung, Probenpunkte der Isotopen-Analysen (LTh 98/13 -51-63)

Anhang 4: $\quad$ Abbildung, Probenpunkte der Isotopen-Analysen (LTh 98/13 -2-12)

Anhang 5: Tabelle, Ergebnisse der Isotopen-Analysen

Anhang 6a-d: Diagramme der Röntgendiffraktometrie

Anhang 7: Tabelle, Ergebnisse der hydrochemischen Analysen

Anhang 8: Kreisdiagramm-Darstellungen, prozentualer Anteil der

Hauptelementverteilungen 


\section{Liste der Abbildungen}

Abb. 1.1: Skizze Australiens und Markierung der Arbeitsgebiete...................................... 11

Abb. 1.2: Lokalität und Probenstandorte Lake Thetis............................................................11

Abb. 1.3: Lokalität und Beprobungsstandorte Lake Clifton, Lake Preston......................... 12

Abb. 1.4: Skizze Lake Walyungup und Beprobungsstandorte............................................. 14

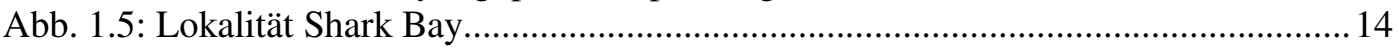

Abb. 3.1: Relativer Vergleich der Mikrobialith-Typen, Lake Thetis.....................................33

Abb. 3.2: Zonengliederung nach Mikrobialith-Typen, Lake Thetis.................................. 37

Abb. 4.1: Gesamtübersicht $\delta^{13} \mathrm{C}$ vers. $\delta^{18} \mathrm{O}$, Kovarianz-Plot der westaustralischen Seen.. 44

Abb. 4.2: Isotope $\delta^{13} \mathrm{C}$ vers. $\delta^{18} \mathrm{O}$, Lake Thetis..................................... 44

Abb. 4.3: Isotope $\delta^{13} \mathrm{C}$ vers. $\delta^{18} \mathrm{O}$,Wachstumsphasen, Stromatolith-Krusten Lake Thetis. 46

Abb. 4.4: Isotope $\delta^{13} \mathrm{C}$ vers. $\delta^{18} \mathrm{O}$, Stromatolith-Krusten, Lake Thetis.......................... 46

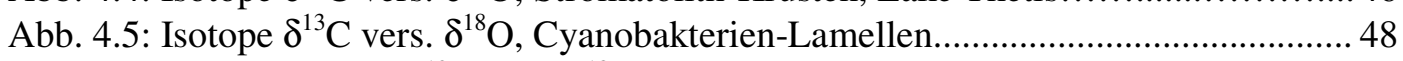

Abb. 4.6: Isotopen-Daten $\delta^{13} \mathrm{C}$ vers. $\delta^{18} \mathrm{O}$, Lake Clifton.............................. 48

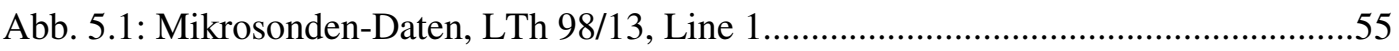

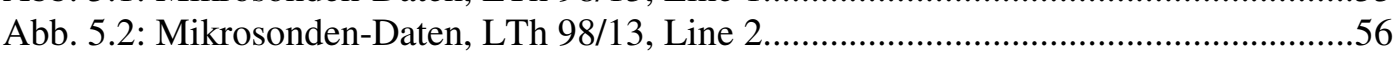

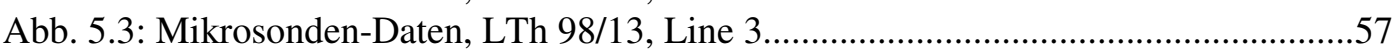

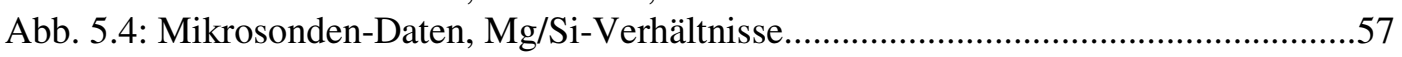

Abb. 5.5: Mikrosonden-Daten, ternäres Ca-Mg-Si-Diagramm..........................................57

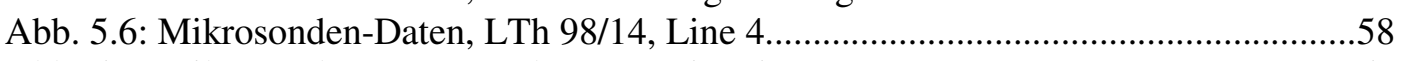

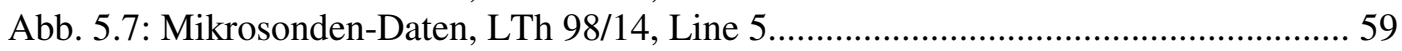

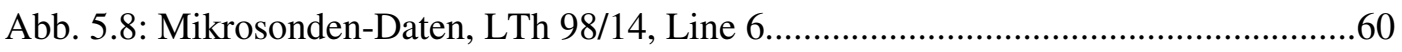

Abb. 5.9: Mikrosonden-Daten, LCl 02/7, Line 8........................................................... 62

Abb. 6.1: Organischer und anorganischer Kohlenstoff, Stromatolith, Lake Thetis............. 64

Abb. 6.2: Biomarker Lake Thetis, Stromatolith, BM 58...................................................67

Abb. 6.3: Biomarker Lake Thetis, Organomikrit-Lage, BM 92........................................69

Abb. 6.4: Biomarker des SPB-reichen Sedimentes, Lake Thetis, BM 99...........................70

Abb. 6.5: Biomarker, Lake Clifton, BM 125....................................... 71

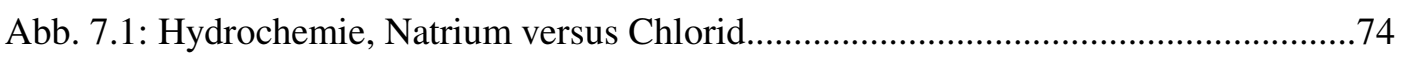

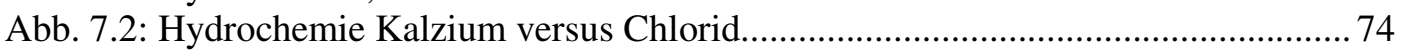

Abb. 7.3: Hydrochemie Kalzium versus Alkalinität..................................................... 78

Abb. 7.4: Hydrochemie Magnesium versus Alkalinität..................................................... 79

Abb. 7.5: Hydrochemie Sulfat versus Chlorid...................................................................... 79

Abb. 8.1: Darstellung der hydrochemischen Modellrechnungen........................................8 83

\section{Liste der Tabellen}

Tab. 5.1: Ergebnisse der röntgenographischen Untersuchungen........................................ 51

Tab. 5.2: Haupt- und Spurenelementverteilung in den Mikrobialithen...............................52

Tab. 5.3: Mittlere geochemische Zusammensetzung einzelner Mineralphasen....................54

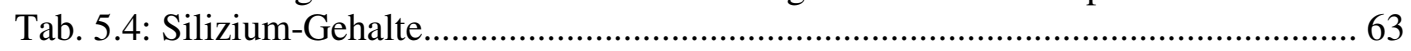

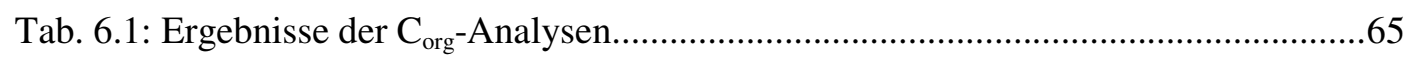

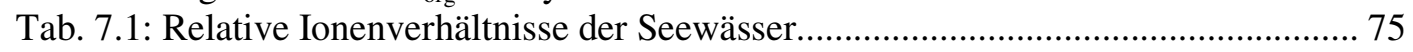

Tab. 7.2: Mg/Ca-Verhältnisse und Sättigungszustände einzelner Mineralphasen................ 81 


\section{Einleitung}

\subsection{Mikrobialithe}

Mikrobialithe sind organosedimentäre Strukturen, die nach BURNE \& MOORE (1987) durch „trapping and binding“ und/oder über Mineralfällungen durch benthische mikrobielle Gemeinschaften gebildet werden. Mikrobielle Karbonate sind seit dem Archaikum ( 3450 Ma) als Bestandteil karbonatischer Sedimente bekannt und waren in flach-marinen Gebieten weit verbreitet. Ihr frühes und periodisch häufiges Auftreten im Verlauf der Erdgeschichte bis in die heutige Zeit und ihr Vorkommen in unterschiedlichsten Milieus, wie marinen, lakustrinen (Hartwasser-, Salzwasserseen und Sodaseen), fluviatilen, speleothemen und thermalen Systemen, verweisen auf ihre besondere evolutionäre Stellung. Die vorliegende Arbeit konzentriert sich auf lakustrine, kalkige Mikrobialithe, die mehrere Meter große, riffähnliche Karbonatkörper (Bioherme) aufbauen können.

Mikrobielle Karbonate werden nach RIDING (1991) hauptsächlich anhand makroskopischer und mikroskopischer Merkmale klassifiziert und in Stromatolithe, Thrombolithe, Travertine und Dendrolithe eingeteilt, die verschiedene, auch ineinander übergehende Gefüge aufweisen. Stromatolithe sind nach KALKOWSKY (1908) laminierte, aufgewölbte Gebilde, die eine reguläre, mehr oder weniger planare, interne Lamination zeigen. Diverse Wuchsformen werden unterschieden: LLH ("laterally linked hemispheroids") und SH ("stacked hemispheroids"). Die Wuchsform der Stromatolithe hängt von der OrganismenVergesellschaftung, der Sedimentzufuhr, der Wassertiefe und -bewegung und der Häufigkeit der subaerischen Exposition ab. Stromatolithe entstehen neben in situ gefälltem Karbonat unter Beteiligung von cyanobakteriellen Matten, Diatomeen, Pilzen und Algen, die die Karbonatfällung direkt beeinflussen, die Sedimentoberfläche durch exopolymere Substanzen (EPS) stabilisieren und verkleben und durch endolithische Mikritisierung vorhandene Gefüge stabilisieren (REID et al., 2000). Neben cyanobakteriell dominierten Matten sind Stromatolithe bekannt, die von nicht-cyanobakteriellen und nicht-phototrophen Gemeinschaften produziert werden (KRETSCHMAR, 1982; KEUPP \& ARP, 1990; BÖHM \& BRACHERT, 1993).

Thrombolithe sind blumenkohlartige, nicht laminierte Gebilde, deren Internstruktur von sogenannten „thrombolite clots“ (unregelmäßige Mikritklumpen) aufgebaut wird. Der Begriff wurde für „,cryptalgal carbonates“ eingeführt (AITKEN, 1967). Thrombolithe sind bereits seit dem Präkambrium bekannt. Charakteristisch sind sie jedoch für das frühe Paläozoikum. 
Die ersten Stromatolithe treten im Archaikum (Warrawoone Group, Westaustralien, 3,5 Ga; Overwachtgroup, Südafrika, 3,4 Ga) auf und gehen vermutlich auf die biologische Aktivität (BUICK \& GROVES, 1995) nicht-cyanobakterieller Gemeinschaften zurück (WALTER, 1994). Für einige archaische Stromatolithe fehlen Belege für eine Bildung unter mikrobieller Aktivität (Lowe, 1994; Grotzinger \& RothmanN, 1996). Ein Maximum an Häufigkeit und Verbreitung bis hin zur Ausbildung von Karbonat-Plattformen ist für das Mittlere und Obere Proterozoikum dokumentiert (AWRAMIK, 1971; WALTER \& HEYES, 1985; GROTZINGER, 1994). Der Rückgang der Stromatolithe im Spät-Präkambrium sowie ihr sporadisches Wiederkehren in späteren Perioden ist noch immer ungeklärt. Ein Zusammenhang lässt sich nur zwischen Blüten marinen, kalkigen Planktons und einem Fehlen der Stromatolithe im späten Mesozoikum finden. Auch wurde das Auftreten Biofilm-abgrasender Metazoen für den Rückgang der Stromatolithe verantwortlich gemacht (AWRAMIK, 1971). Der episodische Rückgang mikrobieller Karbonate im Phanerozoikum wird mit einer Beeinflussung durch Metazoen im Zusammenhang mit fluktuierender, herabgesetzter $\mathrm{CaCO}_{3}$-Sättigung des Meerwassers in Verbindung gebracht (RIDING \& LIANG, 2005). Allerdings sind rezente Stromatolithe (z.B. Bahamas (REID at al., 1995; MACINTYRE et al., 2000; VISSCHER et al., 2000; Paerl et al., 2001), Shark Bay (MCNAMARA, 1992; REID et al., 2003) und Lake Clifton (Westaustralien)) auch in Gebieten mit hoher Metazoenaktivität existent (KONISHI et al., 2001, GARCIA-PICHEL et al., 2004). Lakustrine Stromatolithe sind bereits aus dem Präkambrium bekannt, jedoch erst ab dem Mesozoikum und Kenozoikum häufig (vgl. FLÜGEL, 1982a, S. 43). Es ist weiterhin ungeklärt, ob präkambrische Stromatolithe marinen oder lakustrinen Milieus entstammen.

Rezente Stromatolithe treten sowohl in flach-marinen (Lee Stocking Island, Andros Island, Bahamas; Shark Bay, Westaustralien) als auch in terrestrischen Gebieten auf. Hier konzentrieren sich die z.T. sehr unterschiedlichen Vorkommen auf lakustrine Systeme (Salz-, Soda- und Hartwasserseen). Eine Vielzahl von Sodaseen findet sich in Afrika (z.B. Lake Magadi, Lake Tanganyika), in Indonesien (Satonda Crater Lake, KEMPE \& KAZMIERCZAK, 1990b; ARP et al., 2003), in Europa (Van See, Türkei, KEMPE et al., 1991) und in Westaustralien (Lake Thetis, Lake Clifton, Lake Preston, GREY et al., 1990; BURNE \& MOORE, 1993; MoORE \& Burne, 1994).

Die frühe Photosynthese wurde von anoxygen phototrophen Bakterien (z.B. Purpurbakterien) betrieben, jedoch erst die oxygene Photosynthese durch Cyanobakterien hat zu einer Entwicklung der Sauerstoff-Atmosphäre geführt. Purpurbakterien sind auf reduzierte Wasserstoff-Donatoren $\left(\mathrm{H}_{2} \mathrm{~S}, \mathrm{H}_{2}\right.$ oder organische Verbindungen) angewiesen. Sie entwickeln 
während der Photosynthese keinen Sauerstoff. Cyanobakterien verwerten Wasserstoff als Donator und entwickeln über Pigmente durch Licht Sauerstoff, welcher sich in der Uratmosphäre anreicherte und Grundlage für die spätere Entwicklung des Lebens und der Artenentwicklung ab dem späten Präkambrium war. $\delta^{13} \mathrm{C}$-Untersuchungen an Mikrofossilien aus $2100 \mathrm{Ma}$ und $850 \mathrm{Ma}$ alten Cherts belegen eine Kohlenstofffixierung über den CalvinZyklus (HousE et al., 2000) und liefern somit einen Beleg für die photosynthetische Aktivität der vormals ausschließlich aufgrund ihrer Morphologie als Cyanobakterien interpretierte Mikrofossilien (u.a. SCHOPF, 1968). Anhand morphologischer Gemeinsamkeiten rezenter Strukturen werden Cyanobakterien einem altpräkambrischen Alter von 3,5 Ga zugeordnet (SCHOPF, 1993). Direkte Belege für Cyanobakterien liefern die aus 2,5 Ga alten sedimentären Gesteinen extrahierbaren Hopanoide (Summons et al., 1999). Der Bildungszeitpunkt der Sauerstoffsatmosphäre ist über die ersten cyanobakteriellen Stromatolithe und über die vor 2,1 Ma entstandenen gebänderten Eisenerze (BIFs, Michigan, USA) belegbar. Das vormals im Ozean gelöste Eisen wurde durch den Sauerstoff oxidiert und ausgefällt. Allerdings kann für die BIFs auch eine anaerobe Bildung durch anoxigene, methanotrophe und photosynthetisierende Bakterien angenommen werden, welche Hydrogensulphid und Eisen als Elektronendonatoren verwenden (CANFIELD, 1998) und sich durch C-Isotopen-An- und Abreicherungen in 2,7-2,8 Ga alten Gesteinen belegen lassen (HAYES, 1994).

Einerseits kann ein Wechsel in den $\mathrm{Ca} / \mathrm{Mg}$-Verhältnissen im Ozean das Auftreten und Verschwinden von Cyanobakterien reflektieren (RIDING, 1982), andererseits wird ein Wechsel von globalen Temperaturschwankungen verantwortlich gemacht (RIDING, 1992; 1993; 1994). Cyanobakterielle Kalzifikationsepisoden („CCE“: Cyanobacterial Calcification Episodes) reflektieren Phasen abiotischer Kalzifikation und cyanobakteriellen Wachstums (RIDING, 1992).

Alternativ existieren verschiedene Theorien über einen Wechsel der hydrochemischen Zusammensetzung der Paläo-Ozeane ( RIDING, 1982; KEMPE \& DEGENS, 1985; GROTZINGER, 1990; KeMPE, 1990; KNOLl et al., 1993; KeMPE \& KAZMIERCZAK, 1994; KNAUTH, 1998). Belegen lässt sich ein oszillatorischer Wechsel in der Zusammensetzung des Phanerozoischen Ozeans (HARDIE, 1996). Für das Präkambrium werden von KNAUTH (1998) Salinitäten angenommen, die den heutigen Wert um das 1,5- bis 2-fache übersteigen, wohingegen Holland (1992) Salinitäten an nimmt, die dem heutigen Ozean entsprechen. Basierend auf geochemischen Massenbilanzen, kinetischen und thermodynamischen Betrachtungen und Untersuchungen des Satonda „Soda“-Sees postulieren KEMPE \& DEGENS (1985) und KEMPE \& KAZMIERZCAK (1994) einen frühen, hoch-alkalinen Ozean („Soda-Ozean-Theorie“) mit 
einem hohen ph-Wert und geringen $\mathrm{Ca}^{2+}$ - und $\mathrm{Mg}^{2+}$-Konzentrationen. Im Rahmen der Kontinentbildung wurde das $\mathrm{Na}^{+}$durch Subduktion der Porenwässer und durch Einlagerung in kontinentale Kalksedimente dem Ozean sukzessiv entzogen.

ARP et al. (2001) gehen von einem höheren $\mathrm{Ca}^{2+}$-Gehalt im Phanerozoischen Ozean aus. Die Annahme basiert auf Modellrechnungen, welche durch Untersuchungen an Fluideinschlüssen mariner Halite bestätigt wurde (LOWENSTEIN et al., 2001). Zudem wird das Fehlen von kalzifizierten Cyanobakterien in präkambrischen Stromatolith-Sequenzen durch hohe Konzentrationen an DIC (dissolved inorganic carbon) erklärt (ARP et al., 2001). Das Modell basiert auf Paläo-pCO $\mathrm{PC}_{2}$-Kurven (EKART et al., 1999; BERNER, 1998) und Untersuchungen an Hartwasser-, Soda- und Salzseen.

\subsection{Faktoren der Verkalkungsprozesse}

Die Mechanismen der Karbonatfällung können in abiotische und biotische Faktoren unterschieden werden. Der biotische Einfluss auf das Karbonatgleichgewicht wird in enzymatisch-kontrolliert und biologisch-induziert unterteilt.

\section{Abiotische Faktoren}

Die Verkalkungsprozesse sind an ein übersättigtes Milieu gebunden. In diesem können Änderungen verschiedener physikochemischer Parameter (wie pH, $\mathrm{pCO}_{2}$, Druck- oder Temperaturänderung, Zufuhr externer Ionen $\left.\left(\mathrm{Ca}^{2+}, \mathrm{CO}_{3}{ }^{2-}\right)\right) \mathrm{zu}$ einer Verschiebung des Karbonatgleichgewichtes führen (UsDOwsKi et al., 1979; KELTS \& HsÜ, 1978):

$$
\mathrm{Ca}^{2+}+2 \mathrm{HCO}_{3}^{-} \rightarrow \mathrm{CaCO}_{3}+\mathrm{H}_{2} \mathrm{O}+\mathrm{CO}_{2}
$$

\section{Biotische Faktoren}

Einige Organismengruppen (z.B. Mollusken) fällen Karbonat über intrazelluläre Biomineralisation durch enzymatisch gesteuerte Bereitstellung von Protein-Matrizen. Eine entscheidende Rolle spielen dabei saure Makromoleküle (ADDADI \& WEINER, 1985; 1989). Die Grenzschicht zwischen Organismus und zu bildendem Kristall besteht aus sauren Proteinen und Polysacchariden, die eine sogenannte $\beta$-Faltblattstruktur bilden (ADDADI \& WEINER, 1989; LOWENSTAM \& WEINER, 1989; AdDADI et al., 1990; BosKey, 1996). Carboxyl- $\left(\mathrm{COO}^{-}\right)$und Sulfatgruppen $\left(\mathrm{SO}_{4}{ }^{2-}\right)$ befinden sich so in definierten Abständen innerhalb einer Ebene und bilden kristallographische Grundflächen (für Kalzit 4,99 A und für Aragonit 4,96 ̊). 


\section{Physiologisch induziert}

Eine Verschiebung des Karbonatgleichgewichtes kann durch die physiologische Aktivität von Mikroorganismen erfolgen. Besonders photosynthetische Aktivität führt durch $\mathrm{CO}_{2} / \mathrm{HCO}_{3}^{-}$Aufnahme zur $\mathrm{CaCO}_{3}$-Fällung an cyanobakteriellen Scheiden (MERZ, 1992). Es zeigt sich ein Zusammenhang zwischen der Aufnahme von $\mathrm{HCO}_{3}{ }^{-}$durch Cyanobakterien, der damit verbundenen $\mathrm{OH}^{-}$-Abgabe und der daraus resultierenden Karbonatfällung.

Bakterielle Sulfatreduktion kann zur Alkalinitätsproduktion in Sulfat-reichen Milieus führen (vgl. KEMPE, 1990). Anaerobe Methanoxidation, gekoppelt mit Sulfatreduktion, wird über die Produktion von Alkalinität an Methanquellen als Mechanismus zur Bildung von Karbonatzementen angenommen (PAULL et al., 1992). Der Abbau organischer Substanzen über Nitratreduktion und Ammonifikation resultiert ebenfalls in einer Erhöhung der Alkalinität (Modellreaktion bei KELTS, 1988).

Die Bereitstellung von heterogenen Nukleationsflächen erfolgt durch die CyanobakterienScheiden (PENTECOST \& RIDING, 1986). Neuere Untersuchungen und Modelle zeigen, dass Nukleation (Keimkristallbildung) und Wachstum von Karbonat-Mineralen (Kalzit/Aragonit) durch lösliche und unlösliche organische Matrizen kontrolliert wird, wobei photosynthetische und bakterielle Aktivitäten nur untergeordnet zur Fällung beitragen und die Biofilme lediglich als organische Matrizen dienen. Die EPS haben bei der Organomineralisation (an nicht lebenden, organischen Substanzen) einen biologisch-induzierten Einfluss auf die Verschiebungen des Karbonatgleichgewichtes. Die EPS reduzieren die Diffusionsraten, generieren ein Mikromilieu und komplexieren $\mathrm{Ca}^{2+}$-Ionen an ihren sauren, funktionellen Gruppen (Carboxyl- und Hydroxylgruppen), wodurch initial eine Fällung abgepuffert wird (ARP et al., 1999a). Im Laufe von Degradationsprozessen kommt es zur Freisetzung der Ionen und zu einem Nukleationsprozess auf ungeordneten, sauren Polymeren (ARP et al., 1998), die in kleinere Einheiten zerlegt werden und mit den absorbierten $\mathrm{Ca}^{2+}$-Ionen regelmäßig angeordnete kristallographische Grundflächen bilden können. Es handelt sich hierbei um zufällige Mineralisationsprodukte, also um Organomineralisation (TRICHET \& DÉFARGE, 1995; DÉFARGE \& TRICHET, 1995), die nicht wie bei der Biomineralisation auf organischen Matrizen in definierten Abständen erfolgt, sondern auf nicht lebenden, umstrukturierten makromolekularen Filmen und Aggregaten (REITNER, 1993; REITNER et al., 1995). In situ gebildete Mikrite und Mikritkrusten werden als „Automikrite“ und „Organomikrite“ bezeichent (TRICHET \& DÉFARGE, 1995; NEUWEILER et al., 1999). 


\section{Hydrochemische Parameter}

Grundvoraussetzung für Karbonatfällung in Mikrobialithen ist eine Übersättigung des Systems in Bezug auf das jeweilige Mineral. Die Übersättigung kann einerseits das Milieu des Umgebungswassers, andererseits das Mikromilieu innerhalb der Biofilm-EPS betreffen. Die Cyanobakterien-Kalzifikation ist von den jeweiligen Übersättigungen des Umgebungsmilieus abhängig (PENTECOST \& RIDING, 1986).

Um das Umgebungsmilieu zu charakterisieren, werden neben den Haupt-Anionen und HauptKationen die hydrochemischen Parameter wie die Alkalinität, der pH-Wert sowie der DIC bestimmt.

Die Alkalinität ist analytisch definiert als Menge einer starken Säure, die benötigt wird, um den Überschuss an Basen in einer Lösung zu neutralisieren. Die Karbonatalkalinität (KA) wird beschrieben als:

$$
\begin{aligned}
\mathrm{KA} & =\left[\mathrm{HCO}_{3}^{-}\right]+2\left[\mathrm{CO}_{3}{ }^{2-}\right] \\
{[\mathrm{Alk}] } & =\left[\mathrm{HCO}_{3}^{-}\right]+2\left[\mathrm{CO}_{3}{ }^{2-}\right]+\left[\mathrm{OH}^{-}\right]-\left[\mathrm{H}^{-}\right]
\end{aligned}
$$

Als alternative Definition wird die Alkalinität über den Ladungsausgleich zwischen der Summe der konservativen Kationen und der Summe der konservativen Anionen ausgedrückt (STUMM \& MORGAN, 1996):

$$
\begin{aligned}
{[\mathrm{ALK}] } & =\left[\mathrm{HCO}_{3}{ }^{-}\right]+2\left[\mathrm{CO}_{3}{ }^{2-}\right]+\left[\mathrm{OH}^{-}\right]-\left[\mathrm{H}^{-}\right] \\
& =\left[\mathrm{Na}^{+}\right]+\left[\mathrm{K}^{+}\right]+2\left[\mathrm{Ca}^{2+}\right]+2\left[\mathrm{Mg}^{2+}\right]-\left[\mathrm{Cl}^{-}\right]-2\left[\mathrm{SO}_{4}{ }^{2-}\right]-\left[\mathrm{NO}_{3}{ }^{-}\right]
\end{aligned}
$$

In hochalkalinen Systemen wird die Alkalinität (als Summe von $\mathrm{HCO}_{3}^{-}$- und $\mathrm{CO}_{3}{ }^{2-}$-Ionen) weitestgehend über das $\mathrm{Na}^{+}$bilanziert. Sie werden daher als „Soda-Lakes“ bezeichnet. „Soda“-Seen sind reich an $\mathrm{Na}^{+}$, haben bei hoher Alkalinität auch einen hohen $\mathrm{pH}-$ Wert und enthalten z.T. einen beträchtlichen Anteil an $\mathrm{NaCl}$. $\mathrm{Ca}^{2+}-$ und $\mathrm{Mg}^{2+}$-Ionen werden als Karbonat gefällt, sobald eine bestimmte $\mathrm{Na}^{+}$-ausgeglichene Alkalinität überschritten und der $\mathrm{pCO}_{2}$ gering genug ist (KEMPE \& DEGENS, 1985). Alkaline Salzseen sind daher im Vergleich zum Meerwasser verarmt an $\mathrm{Ca}^{2+}\left(5 \mathrm{mmol} \mathrm{l}^{-1}\right.$ bis $0,1 \mathrm{mmol} \mathrm{l}^{-1}$; Seewasser $\left.10 \mathrm{mmol} \mathrm{l}^{-1}\right)$. Um ein solches System an $\mathrm{CaCO}_{3}$ zu übersättigen, sind geringe $\mathrm{Ca}^{2+}$-Konzentrationen nötig, da das Ionenaktivitätsprodukt zur Präzipitation bereits durch die hohe Karbonat-Konzentration überschritten wird (vgl. EUGSTER \& HARDIE, 1978). Kritisch ist nicht die zur Verfügung stehende Menge an $\mathrm{Ca}^{2+}$, sondern deren konstanter Zufluss, z.B. über fluviatile und sublakustrine Zuflüsse oder meteorische Wässer.

Die chemische Zusammensetzung hypersalinarer Seen wird von $\mathrm{Na}^{+}$und $\mathrm{Cl}^{-}$dominiert. Jede Lösung equivalenter Konzentrationen von $\left(\mathrm{Na}^{+}+\mathrm{K}^{+}\right)>\left(\mathrm{Cl}^{-}+\mathrm{SO}_{4}^{2-}\right)$ und $\left(\mathrm{Ca}^{2+}+\mathrm{Mg}^{2+}\right)<$ 
$\left(\mathrm{HCO}_{3}{ }^{-}+\mathrm{CO}_{3}{ }^{2-}\right)$ wird durch Evaporation alkalin, wenn die Konzentration der Karbonationen $>10^{-2} \mathrm{meq} / \mathrm{L}$ erreicht hat (KEMPE \& KAZMIERCZAK, 1990a). Kalzit, Aragonit und Dolomit präzipitieren, und die Konzentration der Erdalkali-Ionen wird sehr gering.

\subsection{Biofilme und mikrobielle Matten}

Der Term „Biofilm“ bezeichnet benthische Mikrobengemeinschaften, die Wasser-SubstratGrenzflächen in einer Dicke von wenigen Millimetern überziehen. Es handelt sich dabei um monospezifische bis hochkomplexe Populationen, deren Spezies miteinander interagieren und voneinander abhängen (vgl. COSTERTON et al., 1995).

„Mikrobielle Matte“ bezeichnet vertikal laminierte, organosedimentäre Strukturen, welche Ausdehnungen von $1 \mathrm{~mm}$ bis $1 \mathrm{~m}$ erreichen, so dass eine vertikale Lamination bereits makroskopisch sichtbar ist (van GEMERDEN, 1993).

Zur Gruppe der am Aufbau von Biofilmen und mikrobiellen Matten beteiligten Mikroorganismen in dieser Arbeit gehören Cyanobakterien, heterotrophe Bakterien, Diatomeen und Schwefelpurpurbakterien.

Cyanobakterien sind aerobe, phototrophe Bakterien, die wie Algen und höhere Pflanzen photosynthetisch $\mathrm{CO}_{2}$ unter Sauerstoffentwicklung fixieren. Filamentöse Cyanobakterien bestehen aus einem Trichome, welches von einer Mucilage (Schleimschicht) aus Polysacchariden (Exopolysaccharide, EPS) umgeben wird. Die photosynthetisierenden Prokaryonten synthetisieren Chlorophyll a und benutzen meist $\mathrm{H}_{2} \mathrm{O}$ als Elektronendonator bei der Photosynthese (selten $\mathrm{H}_{2} \mathrm{~S}$ als Elektronendonator bei anoxygener Photosynthese). Aufgrund ihrer Zellstruktur, der Mureinzellwand und der 70S Ribosomen handelt es sich bei Cyanobakterien um gram-negative Prokaryonten. Unter den phototrophen Prokaryonten sind die Cyanobakterien die größte, formenreichste und am weitesten verbreitete Gruppe. Aufgrund ihrer Fähigkeit, Stickstoff zu fixieren, können sie auch nährstoffarme Standorte besiedeln. Metabolische Zersetzungsprodukte der Cyanobakterien dienen einer Reihe von Bakteriengruppen als Energie- und Kohlenstoffquelle. Die Gruppe der Cyanobakterien hat eine lange evolutionäre Geschichte, da sie sich bis in das Altpräkambrium zurückverfolgen lässt (KANDLER, 1981; SCHOPF, 1993). Im Zusammenhang mit den Veränderungen der chemischen Zusammensetzung der Erdatmosphäre werden Cyanobakterien als erste photosynthetische Sauerstoffproduzenten angesehen, die einen erheblichen Beitrag zur Entwicklung einer sauerstoffhaltigen Atmosphäre leisteten. 
Heterotrophe Bakterien sind Mikroorganismen, welche ihren Zellkohlenstoff aus organischen Verbindungen beziehen. Sie sind mit Cyanobakterien assoziiert und konsumieren deren metabolische Produkte.

Schwefelpurpurbakterien sind anoxygene, phototrophe Bakterien, die Schwefel als Elektronen-Donator verwenden. Schwefelpurpurbakterien sind an ihrer rot-braunen Färbung und den intrazellulären Schwefelablagerungen $\mathrm{zu}$ erkennen, welche während der Schwefelwasserstoff-Oxidation eingelagert werden.

Diatomeen (Kieselalgen) zählen neben Cyanobakterien, Dinoflagellaten und Algen zu den wichtigsten Primärproduzenten in aquatischen Sedimentationsbereichen. Diatomeen sind einzellige, eukaryotische und photoautotrophe Mikroorganismen mit oxygener Photosynthese. Sie leben solitär oder kommen in losen Zellaggregaten oder distinkten Zellketten, Zellhaufen oder Zellverbänden im Plankton und Benthos vor. Charakteristisch für Diatomeen ist ihre Zellwand, die neben einem Pektinanteil aus Silikat besteht. Die Zellwand verleiht den Diatomeen nicht nur ihre Zellform, sondern wird aufgrund von artspezifischen Ornamentierungen und Porenmustern als taxonomisches Unterscheidungsmerkmal für diese Algengruppe herangezogen. Generell unterscheidet man zwischen den radiärsymmetrischen, zentrischen Diatomeen (Centricae) und den bilateralsymmetrischen, pennaten Diatomeen (Pennatae). Letztere sind in intertidalen Wattsedimenten wie auch in lakustrinen Milieus häufig. Die Schale der Diatomeen besteht aus zwei ineinanderpassenden Hälften (Epitheca und Hypotheca). Bei der Vermehrung durch Teilung bildet jede dieser Hälften ein neues Gegenstück. 


\subsection{Fragestellung}

Anhand von Fallbeispielen soll die rezente Biofilmkalzifikation im Vergleich zu den subfossilen Mikrobialithen untersucht werden. Die Ergebnisse sollen zu einem besseren Verständnis fossiler Gegenstücke lakustriner Mikrobialithe beitragen.

Die in dieser Arbeit verfolgten Ziele und Fragestellungen sind:

- Gibt es rezente Karbonatfällungen und ein Mikrobialith-Wachstum in den Salzseen? Wenn ja: Wie unterscheidet sich das rezente Wachstum in den Seen Lake Thetis und Lake Clifton? Ist die Präzipitation primär durch das hydrochemische Milieu oder die Photosynthese gesteuert?

- Gibt es am Lake Thetis und Lake Clifton unterschiedliche Mikrobialith-Typen?

Wie unterscheiden sich diese in Bezug auf die Verteilung der Haupt- und Spurenelemente in einzelnen Phasen?

- Welche Biomarker kommen vor und sind diese mit vorherigen Arbeiten und anderen Fallbeispielen ähnlicher Milieus identisch? Wie unterscheidet sich das Verteilungsmuster der Biomarker aus den stromatolithischen Karbonaten im Lake Thetis von den Biomarker-Signalen aus dem Schwefelpurpurbakterien-reichen Sediment des Seebeckens?

- Können mit Hilfe der Biomarker-Analysen Rückschlüsse auf fossile Mikrobengemeinschaften gezogen werden? Fossile Proben können aufgrund des geringen Erhaltungspotenzials und der diagenetischen Überprägung wenig oder nicht eindeutig zu interpretierende Signale liefern. Die Untersuchungen des rezenten bis subrezenten Materials sollen helfen, Fehlinterpretationen im fossilen Bericht $\mathrm{zu}$ reduzieren.

- Gibt es Hinweise auf eine Änderung der stabilen Isotopen-Zusammensetzung in jungen Ablagerungen und in unterschiedlichen Cyanobakterien-Lagen? Wie unterscheiden sich die Mikrobialithe der verschiedenen Seen isotopengeochemisch?

- Die westaustralischen Salzseen fungieren im Modell von ARP et al. (2001) als marinevaporatives Endglied im Vergleich zu Frischwasser, Hartwasser- und „Soda“-Seen. Wie stimmen die Ergebnisse nach simuliertem photosynthetischen C-Entzug mit den Resultaten der histologischen Untersuchungen überein? 


\subsection{Arbeitsgebiete}

Als Fallbeispiele zur Untersuchung rezenter Mikrobialith-Bildung wurden fünf verschiedene westaustralische Seen und als marines Beispiel die Shark Bay ausgewählt. Die Seen unterscheiden sich sowohl hinsichtlich der Ausbildung der marginalen Karbonatkörper als auch in der Hydrochemie des Seewassers bzw. ihrer Zuflüsse. Gemeinsam sind allen Seen die klimatischen Bedingungen mit heißen, trockenen Sommern und niederschlagsreichen Wintern.

\section{Lake Thetis}

Der Lake Thetis liegt 3 km südöstlich des Küstenortes Cervantes, 237 km nördlich von Perth, $1,25 \mathrm{~km}$ landeinwärts vom Indischen Ozean (Abb. 1.1) und nördlich des Yanchep Nationalparks. Das Seebecken liegt in einem quartären Basement und besitzt vermutlich ein Alter von 3.000-4.500 Jahren (vgl. GREY et al., 1990). Untersuchungen des Herschell Limestone liefern Fossilienalter von 4.800-5.900 Jahren (PlAYFORD, 1988). Der Lake Thetis besitzt eine Ausdehnung von $300 \mathrm{~m}$ x $150 \mathrm{~m}$ (Abb. 1.2) mit einer maximalen Wassertiefe von $3 \mathrm{~m}$. Die Seespiegelschwankungen sind klimatischen Ursprungs und gehen auf evaporative Prozesse während der Sommermonate und den Eintrag meteorischer Wässer während der Regenzeit zurück. Charakteristischerweise ist die Sedimentoberfläche im Hypolimnion durch ausgedehnte Populationen phototropher Bakterien und Schwefelpurpurbakterien (SPB) bedeckt („flocculent mat“, GREY et al. 1990). Benthische Mikrobengemeinschaften bestehen aus Diatomeen, kokkalen und filamentösen Cyanobakterien und anoxischen Photobakterien (BAULD, 1986). Stromatolith-Bioherme treten entlang der südlichen Uferzone und innerhalb des marginalen Sees auf, erodierte Bioherme sind in Form von Karbonatterrassen besonders am N- und NE-Ufer zu finden. Erste Untersuchungen zur Hydrochemie, Biologie und Sedimentologie finden sich in GREY et al. (1990).

\section{Lake Clifton}

Der Lake Clifton ist ein $18,5 \mathrm{~km}$ langer und bis $\mathrm{zu} 2 \mathrm{~km}$ breiter Salzsee an der westaustralischen Küste (Swan Costal Plain), ca. 100 km südlich von Perth und 1,5 bis 4 km vom Indischen Ozean entfernt gelegen (Abb. 1.1, 1.3). Der See befindet sich innerhalb des Yalgorup Nationalpark, wobei ausschließlich das Seebecken und schmale Uferbereiche geschützt sind. Das E-Ufer ist durch einen Streifen erodierter Bioherme mit konzentrischem Aufbau charakterisiert. Unter permanter Wasserbedeckung liegen kugel- bis kegelförmige thrombolithische Bioherme, welche von BURNE \& MOORE (1993) als Mikrobialithe klassifiziert wurden. Die Form und Verteilung der Thrombolithe scheint an den Zutritt von 


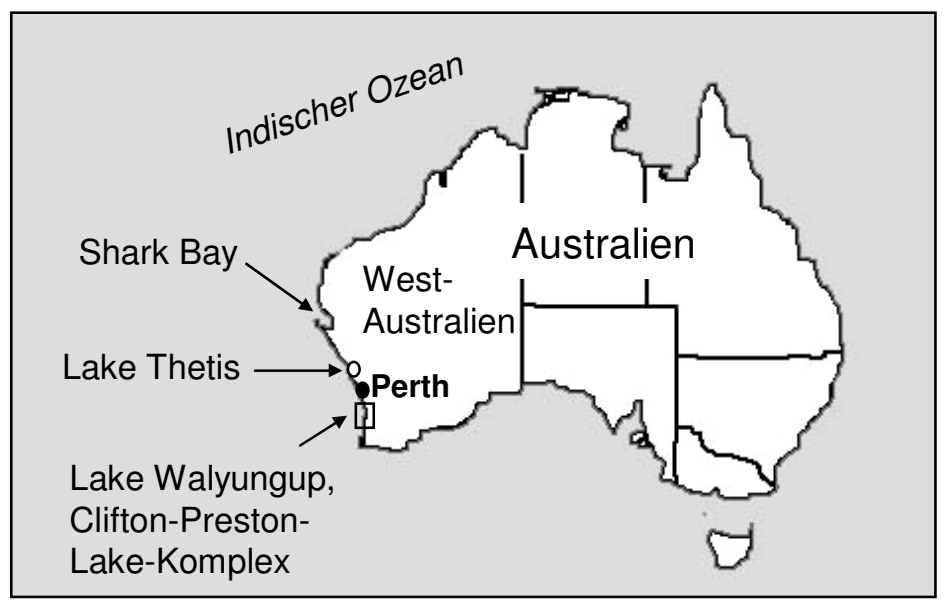

Abb. 1.1: Skizze des australischen Kontinentes. Markiert sind die geographischen Lagen der Arbeitsgebiete: Lake Thetis, Lake Walyungup, Clifton-Preston-LakeKomplex und die Shark Bay.

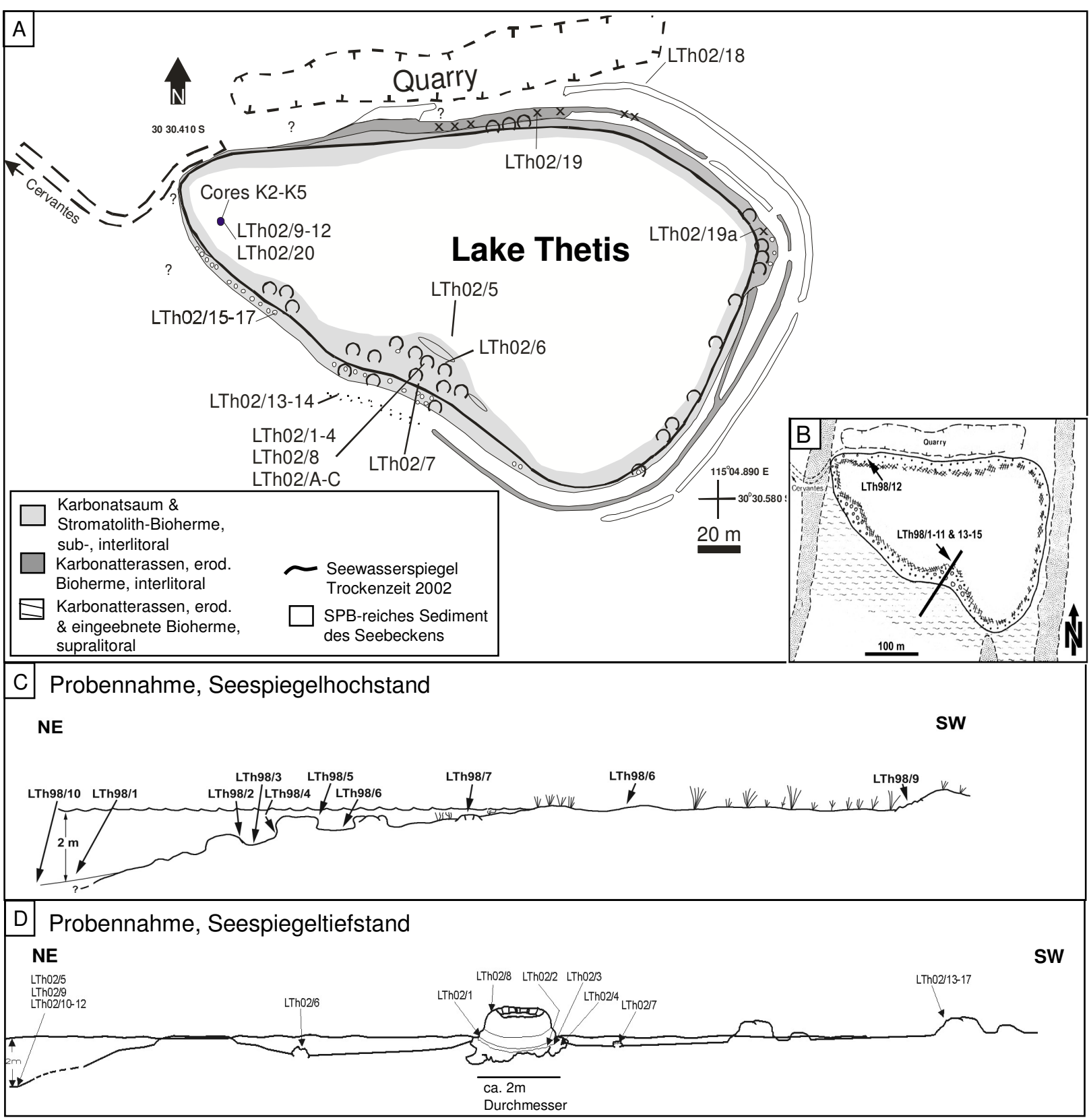

Abb. 1.2: Probenentnahme am Lake Thetis während des Seespiegelhochstandes 2002 (A) und während des Seespiegeltiefstandes 1998 (B; verändert nach Grey et al., 1990). Skizzen von Profillinien des S-Ufers mit Probenpunkten während der Regenzeit (C ) und während der Trockenzeit (D). 

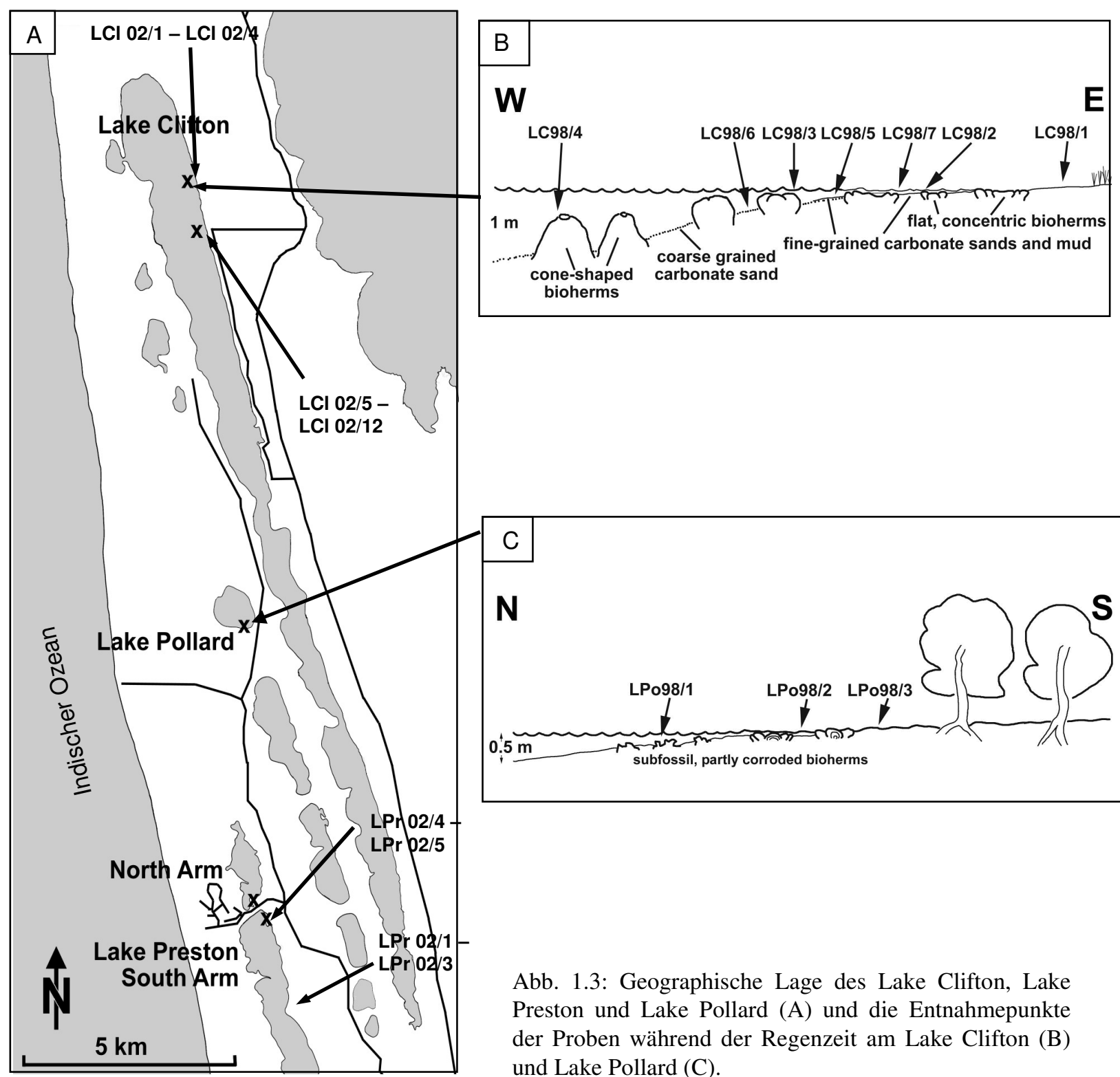

Abb. 1.3: Geographische Lage des Lake Clifton, Lake Preston und Lake Pollard (A) und die Entnahmepunkte der Proben während der Regenzeit am Lake Clifton (B) und Lake Pollard (C). 
Karbonat- und Bikarbonat-haltigem Grundwasser gekoppelt zu sein. Die Thrombolithe sind ein Produkt von Cyanobakterien der Gattung Scytonema sp. (MCNAMARA, 1992). Die Mikrobialithe werden als morphologische Analoga kambrischer Stromatolithe des Cryptozoon proliferum-Typ angesehen (BURNE \& MOORE, 1993). Biomarker-Analysen der fossilen, interlitoralen Bioherme charakterisieren Cyanobakterien und hauptsächlich Diatomeen als Primärproduzenten des organischen Materials (THIEL, 1997).

\section{Lake Pollard und Lake Preston}

Der Lake Preston und der Lake Pollard liegen zwischen dem Lake Clifton und dem Indischen Ozean (Abb. 1.3). Der Lake Pollard befindet sich auf mittlerer Höhe des Lake Clifton und hat einen Durchmesser von ca. $1 \mathrm{~km}$.

Der Lake Preston liegt ca. 4 km südlich vom Lake Pollard und hat eine Breite von ca. 1,5 km. Der See zeichnet sich durch einen $2 \mathrm{~km}$ langen Nordarm und einen $22 \mathrm{~km}$ langen Südarm aus, die in Regenzeiten eine Verbindung durch einen Überlauf zueinander haben und bei Trockenzeiten voneinander isolierte Seebecken darstellen. Proben wurden an der E-Seite des nördlichen Ufers am Lake Preston genommen.

\section{Lake Walyungup}

Der Lake Walyungup liegt 2,3 km östlich des Indischen Ozeans und ca. $40 \mathrm{~km}$ südlich von Perth. Der See erstreckt sich in einer Breite von 0,7 km und einer Länge von 3,6 km (Abb. 1.4). Bei Niedrigwasser ist der See dreigeteilt. Weite Teile des $\mathrm{N}$ - und S-Teils sind trockengefallen, nur der mittlere Teil ist wasserführend mit einem Ufersaum von ca. $300 \mathrm{~m}$. Neben aragonitischer und dolomitischer Mineralogie zeichnen sich die Thrombolithe durch einen hohen Anteil an Hydromagnesit aus (COSHELL et al., 1998).

\section{Shark Bay}

Die Shark Bay liegt 800 km nördlich von Perth an der Westküste Australiens (Abb. 1.5). Im Jahr 1998 wurden Proben von Carbla Point und Hamelin Pool, im Jahr 2002 ausschließlich von Hamelin Pool genommen. Im Intertidal bis Subtidal umsäumen zahlreiche Stromatolithe die Küstenlinie am südlichen Bereich der hypersalinaren, marin-geprägten Shark Bay. REID et al. (2003) beschreiben mikrobielles, „trapping and binding“ im Zusammenspiel mit mikrobieller Mikrit-Fällung als Kriterien für die Stromatolith-Bildung. 

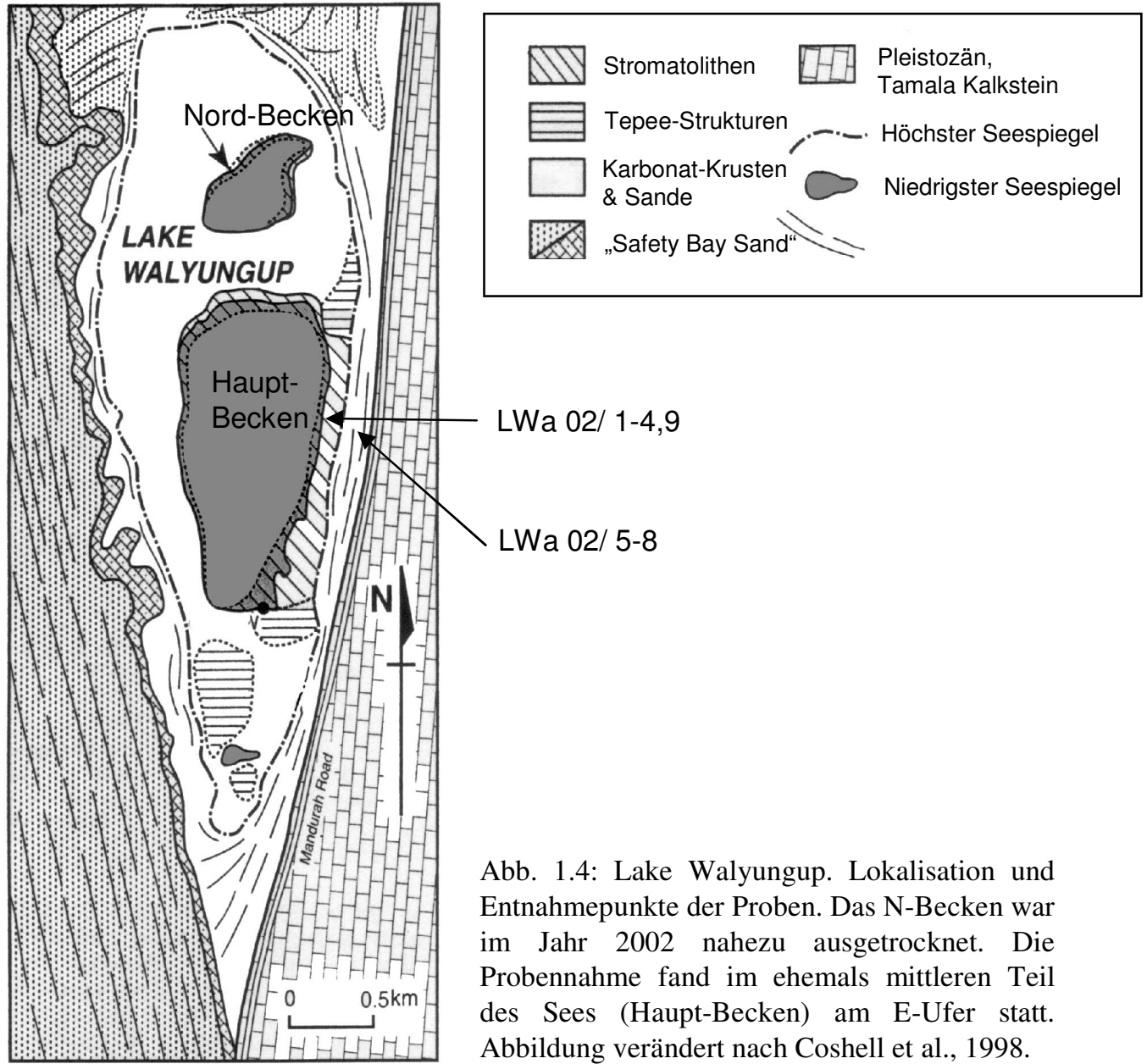

LWa 02/ 1-4,9

LWa 02/ 5-8

Abb. 1.4: Lake Walyungup. Lokalisation und Entnahmepunkte der Proben. Das N-Becken war im Jahr 2002 nahezu ausgetrocknet. Die Probennahme fand im ehemals mittleren Teil des Sees (Haupt-Becken) am E-Ufer statt. Abbildung verändert nach Coshell et al., 1998.

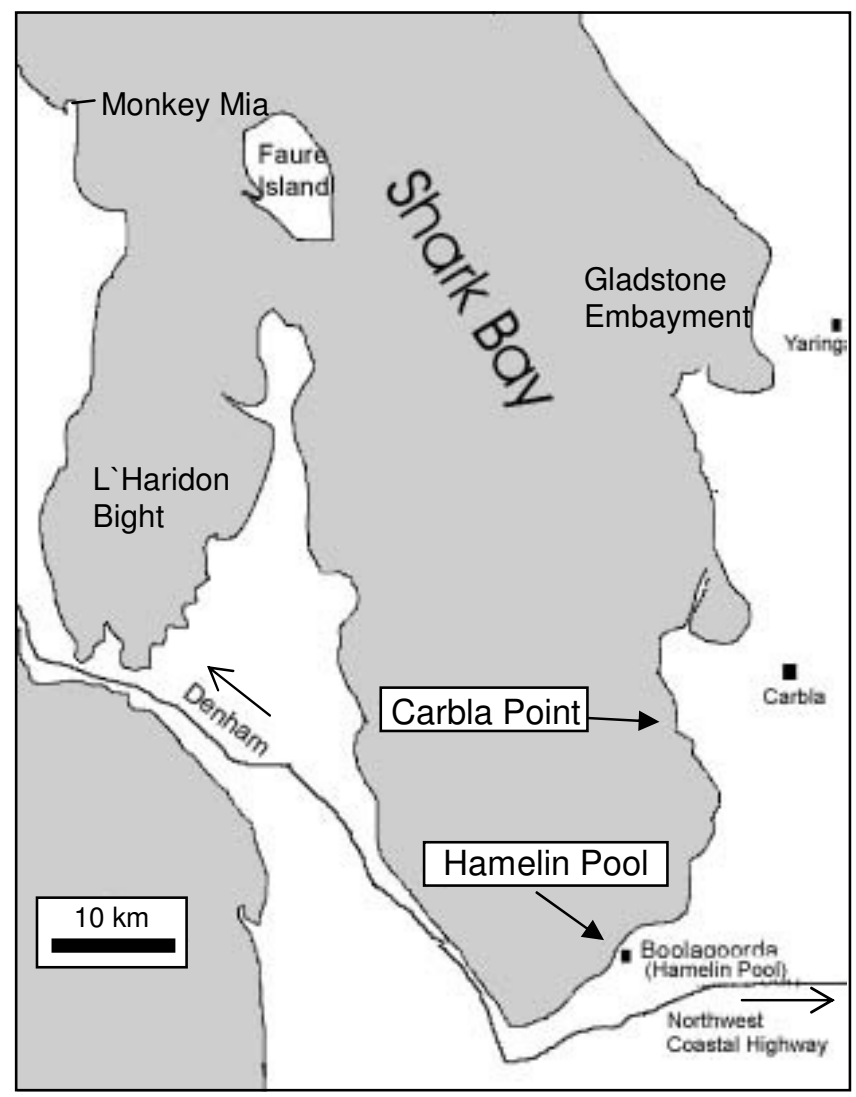

Abb. 1.5: Lokalisation der Proben-punkte an der Shark Bay. Die Proben der Telegraph Station - Hamelin Pool (1998 und 2002) wurden ca. 200 bis $400 \mathrm{~m}$ nord-östlich der Uferzone genommen. Proben von Carbla Point, L`Haridon und Gladstone wurden ausschließlich 1998 genommen. Zur geographischen Lage der Shark Bay siehe Abb. 1.1. 


\subsection{Material und Methoden}

Die fünf westaustralischen Salzseen (Lake Thetis, Lake Clifton, Lake Preston, Lake Pollard, Lake Walyungup) sowie die marin-geprägte Lagune der Shark Bay wurden während des australischen Spätsommers im April 2002 kurz vor der Regenzeit beprobt. Zusätzlich wurden Proben einer Kampagne im August 1998 zur Trockenzeit genommen (Anhang 1-2). Neben kalzifizierten Proben der fossilen und subfossilen Mikrobialithe und rezenten Biofilmen wurden Proben des Umgebungswassers genommen.

\section{Histologie}

Proben der Biofilm-bedeckten Oberflächen und Sedimente wurden in 4 Probenteile (ca. 4 x 4 $\mathrm{cm})$ geteilt. Ein Teil der Proben wurde in 4\%igem Formalin fixiert und in PBS-gepuffertem Standortwasser (filtriert mit $<0,25 \mu \mathrm{m}$ Cellulose-Acetatfilter) kühl gelagert. Ein weiterer Probenteil wurde vor der Formalinfixierung in Calcein getränkt und mehrmals gespült, um die Autofluoreszenz durch das Calcein zu vermindern. Ein weiterer Probenteil wurde mit Glutaraldehyd (4\%) fixiert. Der vierte Probenteil wurden mit Formalin fixiert und in 50\% EtOH und PBS-gepuffertem Wasser gelagert. Im Labor wurden die Formalin-fixierten Proben schrittweise entwässert (15-30-50-70-90-95-100\%EtOH), in Acrylharz LR-White (1:2, 2:1, EtOH : LR White) eingebettet, bei $60^{\circ}$ über Nacht ausgehärtet, mit Hardpart Microtome in 300-500 $\mu \mathrm{m}$ dicke Scheiben geschnitten und mit der Flächenschleifmaschine auf 30-50 $\mu \mathrm{m}$ heruntergeschliffen. Die Untersuchung der Schliffe und deren Dokumentation mittels digitaler Kamera (VISICAM-color CCD Kamera, PCO Computer Optics GmbH) erfolgte am Zeiss Axioplan Mikroskop mit Epifluoreszens-Einrichtung und Phaseninterferenz-Kontrast (DIC, differential interference contrast, Nomarski optics). Dafür wurden die Filter-Sätze Zeiss 01 (BP 365/12, LP 397), Omega XF 23 (BP 485/22, BP 535/35) sowie Omega XF 37 (BP 546/10, BP580/30) verwendet. Die digitale Verarbeitung der Bilder erfolgte über das Programm Metamorph ${ }^{\circledR}$-Imaging Software und Adobe ${ }^{\circledR}$ Photoshop ${ }^{\circledR}$ 6.0.

\section{Dünnschliffe}

Von den Gesteinsproben wurden Dünnschliffe in der Größe 10 x 15 cm $(7$ x Lake Thetis, 8 x restliche Seen) und 10 x 7,5 cm (8 x Lake Thetis, 7 x restliche Seen) hergestellt, die mittels Schleifkornfreier Flächenschleifmaschine auf eine Dicke von 50-100 $\mu \mathrm{m}$ heruntergeschliffen und unter Binokular und Mikroskop (Zeiss, Axioplan) untersucht wurden. 


\section{Röntgen-Untersuchungen}

Zur Bestimmung der mineralogischen Zusammensetzung der karbonatischen Gesteinsproben wurden röntgenographische Untersuchungen an pulverisiertem Probenmaterial vorgenommen. Die Messungen wurden am Geowissenschaftlichen Zentrum der Universität Göttingen (Abteilung Experimentelle und Angewandte Mineralogie) durchgeführt.

\section{Elementgehalts-Bestimmungen}

Von 53 karbonatischen Proben (identisch mit den Isotopen-Proben) wurden Säureaufschlüsse mit 2n $\mathrm{HCl}$ gemacht. Dazu wurden $50 \mathrm{mg}$ Probe in $4 \mathrm{ml} \mathrm{2n} \mathrm{HCl}$ aufgelöst und auf einer Heizplatte erhitzt. Über Blaubandfilter wurden die Proben in $100 \mathrm{ml}$ Messkolben mit Wasser $_{\text {demi }}$ überführt. Die Proben wurden mittels AAS und ICP-OES auf die Haupt- und Spurenelementgehalte untersucht. 27 Proben wurden mit 5\%iger Natrium-Karbonat-Lösung aufgeschlossen und der Si-Gehalt wurde photometrisch nach GRASSHOFF et al. (1983) bestimmt.

\section{ElektronenstrahImikrosonden-Analyse}

Um einzelne Mineralphasen zu charakterisieren, wurde die chemische Zusammensetzung (Elemente $\mathrm{Ca}, \mathrm{Mg}$, Si, Sr, Fe, Mn, Ba, Na, K, Al, Ti, C, H) zweier ausgesuchter subfossiler Stromatolithe des Lake Thetis und einer rezenten Thrombolith-Kruste des Lake Clifton über die Elektronenstrahlmikrosondenanalyse (EMS) ermittelt. Dazu wurden LR-Whiteeingebettete, polierte und mit Kohlenstoff bedampfte Proben in Größe von 48 x 24 mm verwendet. Sieben Einzelpunkte und sieben Lines mit insgesamt 419 Einzelmesspunkten wurden am Gerät JEOL JXA 8900 RL Elektronenstrahlmikrosonde (mit $15 \mathrm{kV}$ und $12 \mathrm{nA}$ ) am Geologischen Zentrum der Universität Göttingen (GZG), Abteilung Geochemie gemessen. In den karbonatischen und silikatischen Bereichen wurden die Elemente jeweils mit einem karbonat- bzw. silikatspezifischen Programm gemessen. Die Angaben erfolgen in Oxid-Form. Mittels Silikat-Programm wurden die Elemente der Oxide $\mathrm{SiO}_{2}, \mathrm{MgO}, \mathrm{K}_{2} \mathrm{O}, \mathrm{CaO}, \mathrm{FeO}$, $\mathrm{Al}_{2} \mathrm{O}_{3}, \mathrm{Na}_{2} \mathrm{O}, \mathrm{SrO}, \mathrm{TiO}_{2}, \mathrm{MnO}, \mathrm{H}_{2} \mathrm{O}$ ermittelt, während die Elemente der Oxide $\mathrm{SrO}, \mathrm{MgO}$, $\mathrm{SiO}_{2}, \mathrm{BaO}, \mathrm{CaO}, \mathrm{FeO}, \mathrm{MnO}, \mathrm{CO}_{2}$ mittels Karbonat-Programm ermittelt wurden. Die Nachweisgrenzen der Oxidformen in Gew.-\% sind für die Elemente $\mathrm{Sr}$, Mn und Fe in Bezug auf die beiden Messprogramme unterschiedlich. Im Silikatprogramm liegen die Nachweisgrenzen für die Oxide $\mathrm{SiO}_{2}, \mathrm{MgO}, \mathrm{SrO}, \mathrm{CaO}, \mathrm{FeO}, \mathrm{MnO}$ und $\mathrm{K}_{2} \mathrm{O}$ nicht über 0,06 Gew.-\% und für $\mathrm{Al}_{2} \mathrm{O}_{3}, \mathrm{Na}_{2} \mathrm{O}_{3}$ und $\mathrm{TiO}_{2}$ nicht über 0,09 Gew.-\%. Beim Karbonatprogramm liegt die Nachweisgrenze für $\mathrm{SiO}_{2}$ nicht über 0,08 Gew.-\%, für $\mathrm{SrO}$ und $\mathrm{CaO}$ nicht über 0,04 Gew.-\% und für $\mathrm{MgO}, \mathrm{FeO}$ und $\mathrm{MnO}$ nicht über 0,06 Gew.-\%. 
Für das Detektionslimit gilt

$$
\mathrm{I}_{\mathrm{dl}}=2 \sigma_{\mathrm{BG}}
$$

mit Idl $=$ Nettoimpulsrate am Detektionslimit und $\sigma_{\mathrm{bg}}=$ Standardabweichung der Untergrundimpulsrate (Mehrfachmessungen).

In den untersuchten Schliffen konnten mittels Epifluoreszenz-Mikroskopie (Metamorph®) die Messpunkte kontrolliert und optisch wiedergegeben werden. Die Bildbearbeitung erfolgte über Adobe ${ }^{\circledR}$ Photoshop®6.0. Die Durchmesser der durch den Elektronenstrahl entstandenen Messkrater betragen 10-15 $\mu \mathrm{m}$.

\section{Stabile Isotope}

Zur Bestimmung der stabilen Isotope wurden 53 Karbonat-Proben der subfossilen Stromatolithe und Sedimente (Tab. 4) mittels Diamantbohrer und Achatmörser pulverisiert. Als repräsentative Proben wurden verschiedene Stromatolith-Typen, Wechselfolgen von Cyanobakterien-Lagen, „Organomikrit“-Krusten, Thrombolithe sowie Gesamtgesteins-Proben untersucht. Zudem wurden Spherulith-Proben analysiert, die aus Kernen stammen, welche von GREY et al. (1990) gezogen und Prof. Reitner freundlicherweise zur Verfügung gestellt wurden.

Die Isotopen-Verhältnisse $(\mathrm{R}=18 \mathrm{O} / 16 \mathrm{O}$ bzw. $\mathrm{R}=13 \mathrm{C} / 12 \mathrm{C})$ werden als $\delta^{13} \mathrm{C}$ - und $\delta^{18} \mathrm{O}$ Wert angegeben und ergeben sich aus

$$
\delta^{13} \mathrm{C} \text { bzw. } \delta^{18} \mathrm{O}=\left[\left(\mathrm{R}_{\text {Probe }} \mathrm{x} \mathrm{R}_{\text {Standard }} / \mathrm{R}_{\text {Standard }}\right) \times 1000[\% \mathrm{\%}] .\right.
$$

Die Werte beziehen sich auf den PDB-Standard. Die Messungen wurden am Institut für Geologie und Mineralogie der Universität Erlangen-Nürnberg von Dr. M. Joachimski durchgeführt.

\section{Kohlenstoff- und Stickstoff-Bestimmungen}

Die Bestimmung des organischen Kohlenstoffs und des Gesamtkarbonat-Gehaltes erfolgte am Leco CHN-1000 Analyser (GZG, Abteilung Umwelt- und Sedimentgeologie). 50 mg der pulverisierten Probe wurden für den Gesamtkohlenstoff-Gehalt benötigt. 100 mg Probe wurde zur Bestimmung des organischen Kohlenstoffes mit 2n Phosphorsäure vorbehandelt. Anorganischer Kohlenstoff wurde aus der Differenz beider Gehalte ermittelt. Alle Proben wurden in Doppelbestimmung gemessen. Zur Kalibrierung wurden EDTA und Leco Kohlenstoff-Standard verwendet. 


\section{Biomarker}

Die karbonatischen Mikrobialith-Proben (2 vom Lake Thetis, 1 vom Lake Clifton) wurden mechanisch zerkleinert, mit Aceton gewaschen und durch Zugabe von 2n $\mathrm{HCl}$ sukzessiv aufgelöst und anschließend mit $\mathrm{H}_{2} \mathrm{O}_{\text {demi }}$ gewaschen und getrocknet.

Um estergebundene Lipidkomponenten freizusetzen, wurde die Probe einer alkalischen

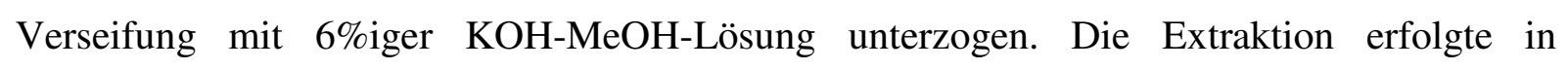
$\mathrm{CH}_{2} \mathrm{Cl}_{2} / \mathrm{CH}_{3} \mathrm{OH}(3: 1 ; \mathrm{v}: \mathrm{v})$ im Ultraschallbad in dreimaliger Wiederholung $\left(70^{\circ} \mathrm{C}, 2 \mathrm{~h}\right)$. Der Überstand wurde auf einen pH von 2 eingestellt. Durch Chromatographie (Silika-Gel 60, Merck) wurde nach unterschiedlichen Polaritäten in Kohlenwasserstoffe (mittels $n$-Hexan), Alkohole, Ketone (mittels $\mathrm{CH}_{2} \mathrm{Cl}_{2}$ ) und in die polare Fraktion der Karbonsäuren (mittels $\left.\mathrm{CH}_{2} \mathrm{Cl}_{2} / \mathrm{MeOH}\right)$ getrennt. In der Fraktion der Kohlenwasserstoffe wurde der Schwefel mit aktivem Kupfer quantitativ entfernt. Durch eine Derivatisierung wurden die Alkohole in ihre Acetate und die Karbonsäuren in ihre Methylester überführt. Vor der Messung im GC-MS wurden die Fraktionen in 0,5 ml des internen Standards Cholestan aufgenommen.

Zur Bestimmung der $\Delta$-Positionen wurde an der Me-Fraktion eine DMDS-Derivatisierung mit Dimethylsulfid vorgenommen. Die Proben wurden am GaschromatographMassenspektrometer (GC-MS) des GZG, Abteilung Geobiologie gemessen und von Prof. J. Peckmann (Forschungszentrum für Ozeanränder, Universität Bremen) ausgewertet.

\section{Hydrochemie}

Hydrochemische Proben wurden an den S-Ufern des Lake Thetis und des Lake Pollard (nur 1998) und an den E-Ufern des Lake Clifton und des Lake Preston genommen. Die Probenahme am Lake Walyungup fand zur Trockenzeit im mittleren Teil des Sees statt. An der Shark Bay wurden in der Regenzeit Proben von Carbla Point, L`Haridon, Monkey Mia und Hamelin Pool, zur Trockenzeit ausschließlich von Hamelin Pool genommen (Abb. 3). Es wurde jeweils bei niedriger Tide beprobt.

Die Bestimmung der Parameter Leitfähigkeit (electrical conductivity, EC), Temperatur, pH und Redox-Potential wurden im Gelände vorgenommen. Der $\mathrm{pH}$ wurden mit einem WTW 330i pH-Meter (Hanna Instr.), das Redoxpotential mit einem 91 pH-Meter (WTW Co.) und die Konduktivität mit einem WTW LF 323 gemessen. Die Eichung der pH-Elektroden erfolgte über NBS-Puffer. Die Bestimmung der Gesamtalkalinität wurde im Gelände mittels Handtitrator und 1,6 n Schwefelsäure-Kartuschen (Hach Co.) vorgenommen. Gelöster Sauerstoff wurde nach der Winkler-Methode mittels Glasflaschen (Winkler-Flaschen) und Hach-Titrator mit $0,2 \mathrm{n}$ Natrium-Thiosulfat-Kartuschen ebenfalls direkt nach der 
Probennahme bestimmt. Für die Kationen-Analysen wurden die Proben über Glasfaser-Filter (Whatman GF/F) mit nominaler Porengröße von $0,7 \mu \mathrm{m}$ gefiltert und mit $\mathrm{HNO}_{3}$ fixiert. Im Labor wurden die Kationen Kalzium, Magnesium, Natrium, Kalium und Strontium mit AtomAbsorptions-Spektrometrie (AAS; Philips Pu 9200X) und ICP-OES gemessen. Phosphat, Kieselsäure, Nitrit und Ammonium wurden im Gelände mittels Photometer (LASA 2-plus, Dr. Lange Co.) nach GRASSHOFF et al. (1983) analysiert. Chlorid wurde titrimetrisch mit $\mathrm{AgNO}_{3}$ bestimmt. Sulfat-Gehalte wurden über Gesamtschwefel-Bestimmung mit der ICPOES ermittelt. Parameter des Karbonatsystems $\left(\mathrm{pCO}_{2}\right.$ und Sättigung in Bezug auf Kalzit und Aragonit) wurden mit dem Programm PHREEQC (PARKHURST \& APPELO, 1999) kalkuliert.

Aus der hydrochemischen Zusammensetzung der Seewässer kann der Sättigungszustand berechnet werden: $\quad \Omega=\mathrm{IAP} / \mathrm{LP}$

Der Sättigungsindex ist gegeben durch: $\quad \mathrm{SI}=\log (\mathrm{IAP} / \mathrm{LP})$,

wenn IAP das Ionenaktivitätsprodukt und LP das Löslichkeitsprodukt der korrespondierenden festen Mineralphase beschreibt (STUMM \& MORGAN, 1996). Das Löslichkeitsprodukt LP ist charakterisiert über: $\quad \mathrm{LP}=\left[\mathrm{Ca}^{2+}\right] \times\left[\mathrm{CO}_{3}{ }^{2-}\right]$.

Die GPS-ermittelten Koordinaten zur Lokalisation der Entnahmepunkte der hydrochemischen Proben von 2002 sind:

Lake Thetis $\mathrm{H} 01$, Lake $1,10 \mathrm{~cm}$ Tiefe: $\quad 30^{\circ} 30^{`} 515 \mathrm{~S}, 115^{\circ} 04^{`} 750 \mathrm{E}$ H02, Lake 2, $70 \mathrm{~cm}$ Tiefe: $\quad 30^{\circ} 30^{\circ} 497 \mathrm{~S}, 115^{\circ} 04^{`} 695 \mathrm{E}$ H03, Lake 3, 1,5 m Tiefe: $\quad 30^{\circ} 30^{`} 492 \mathrm{~S}, 115^{\circ} 04^{`} 700 \mathrm{E}$ H04, Lake 4, 2 m Tiefe: $\quad 30^{\circ} 30^{\circ} 492 \mathrm{~S}, 115^{\circ} 04^{`} 700 \mathrm{E}$ H05, 2,3 m Tiefe: $\quad 30^{\circ} 30^{\circ} 455 \mathrm{~S}, 115^{\circ} 04^{`} 650 \mathrm{E}$ H06, 2, 2 m Tiefe: $\quad 30^{\circ} 30^{\circ} 455 \mathrm{~S}, 115^{\circ} 04 \div 650 \mathrm{E}$

Lake Clifton H01, Pond 1: $\quad 32^{\circ} 44^{`} 540 \mathrm{~S} ; 115^{\circ} 39^{`} 087 \mathrm{E}$ H02, Pond 2: $\quad 32^{\circ} 44^{\circ} 541 \mathrm{~S} ; 115^{\circ} 39^{\circ} 084 \mathrm{E}$ H03, Lake 1: $\quad 32^{\circ} 444^{`} 542 \mathrm{~S} ; 115^{\circ} 39^{\circ} 079 \mathrm{E}$ H04, Lake 2: $\quad 32^{\circ} 44^{`} 794 \mathrm{~S} ; 115^{\circ} 39^{`} 139 \mathrm{E}$

Aus dem Jahr 1998 existieren keine GPS-Daten. Die Probenpunkte sind allerdings nahezu identisch.

\section{Sedimentkerne}

Es wurden 3 Sedimentkerne des westlichen Seebeckens des Lake Thetis mit Hilfe von ca. $50 \mathrm{~cm}$ langen Plexiglasrohren mit einem Durchmesser von $10 \mathrm{~cm}$ gezogen. Die Wassertiefe betrug 1,5 bis $2 \mathrm{~m}$. Die Kerne wurden direkt nach der Entnahme fotografiert. Im Labor wurden die Kerne LTh K2 und LTh K5 geöffnet. In Abständen von ca. 2 cm (K5) und ca. $5 \mathrm{~cm}$ (K2) wurden Proben für histologische Untersuchungen, Element- und IsotopenUntersuchungen entnommen. 


\section{Rezentes Mikrobialith-Wachstum}

Im Hinblick auf rezente Verkalkungsmuster und damit zusammenhängendes MikrobialithWachstum wurden die Oberflächen der sub- bis interlitoralen Mikrobialithe in den Salzseen und die intertidalen Stromatolithe der Shark Bay bei Hamelin Pool untersucht. Dazu wurden in LR-White eingebettete und zum Teil mit Calcein eingefärbte Dünnschnitte im Hellfeld mittels Epifluoreszenz-Einrichtung sowie zwischen gekreuzten Polarisatoren mikroskopiert. Die Proben repräsentieren zum einen den Zustand während der Regenzeit 1998 (Seespiegelhochstand) und zum anderen während der Trockenzeit 2002 (Seespiegeltiefstand).

\subsection{Lake Thetis}

Die mikrobiellen Matten der Stromatolithe bestehen nach GREY et al. (1990) aus fünf Typen und zeigen eine Zonierung des Seeufers. Ein Vergleich dieser Typen ist aufgrund der damals vorgenommen Probenbehandlung (Dekalzifizierung mittels EDTA) nicht möglich. Die benthischen Mikrobenmatten der vorliegenden Arbeit wurden in situ eingebettet, um das Karbonatgerüst nicht zu zerstören und Zusammenhänge zu den Biofilmen dokumentieren zu können.

\section{Regenzeit}

Diatomeen haften an der Biofilm- und Karbonatoberfläche in Form von senkrecht aufgestellten Schalen. Die benthischen Mikrobenmatten der Stromatolith-Oberflächen sind zudem Scytonema-dominiert und bestehen auch aus Gloeocapsa-Kolonien und untergeordnet Phormidium sp.. Scytonema-Filamente haben einen Durchmesser von $20 \mu \mathrm{m}$, wobei das Trichome einen Durchmesser von $7 \mu \mathrm{m}$ aufweist. Je nach Anschnitt beträgt die Länge maximal $140 \mu \mathrm{m}$, meist jedoch $100 \mu \mathrm{m}$. Gloeocapsa sp. hat einen Durchmesser von durchschnittlich 2,0-2,5 $\mu \mathrm{m}$.

Intakte, erekte Scytonema-Filamente repräsentieren den äußeren Biofilmrand im Kontakt zum Seewasser. Partiell sind Scytonema-Lagen erkennbar, in denen die Filamente erekt orientiert sind und von Karbonatpräzipitaten eingeschlossen werden (Taf. 3/E-H). Charakteristisch ist die darunter liegende, intakte Scytonema-Lage, die - entsprechend den jüngsten Filamenten weder Verkalkungen der Filamente noch der Scheiden aufweist (Taf. 3/A-C). Entsprechende Verkalkungsmuster sind auf Biofilme der knotigen, schwarz-grünen Oberflächen (,nodular mat"“) des lateralen Bioherm-Saumes (Zone II, Taf. 1/B) beschränkt und treten nur partiell, aber oberflächenparallel auf. Sie werden aufgrund der intakten und nicht-kollabierten Filamente als Resultat rezent verlaufender Karbonatpräzipitation angesehen.

In subrezente, oberflächennahen Bereichen sind in Karbonat eingebettete Scytonema sp. 
häufig, welche ein charakteristisch braungefärbtes, mikrokristallines Zentrum zeigen (Taf. 3/D). Auf mikrokristallinen Aragonit-Lagen wachsen Scytonema-Filamente erekt auf. Diesen folgt eine Zone von Gloeocapsa-Kolonien, die von einer äußeren Lage senkrecht aufgestellter Diatomeen-Schalen abgeschlossen wird. (Taf. 4/A).

Äußere, mikrokristalline Aragonite, welche z.T ungleichmäßig laminiert sind, weisen Anbohrungen auf und gehen vermutlich auf endolithische Cyanobakterien (,HyellaMorphotyp“) zurück.

\section{Trockenzeit}

Rezente Biofilmgemeinschaften sind Gloeocapsa- und Entophysalis-dominiert (Taf. 4/B-C, 4/G, 5/B). Rezente Verkalkungen, welche als „Mikrostromatolith“ aus dunkelgrauem, mikrokristallinem Aragonit bestehen, bilden sich im direkten Kontakt zu den GloeocapsaKolonien (Taf. 4/D-E, 4/G). Heterotrophe Bakterien an den äußeren Polysaccharid-Hüllen des Entophysalis-Biofilms stellen die Startpunkte der Fällung dar (REITNER et al., 1996). In den Proben sind heterotrophe Bakterien als Zwickelfüllung innerhalb der Gloeocapsa-reichen Biofilme zu erkennen (Taf. 6/E-F), welche allerdings nur im Kontakt zur Karbonatbasis eine Fällung beeinflussen. Im intakten Biofilm selbst findet keine Fällung statt, auch nicht im Bereich der angesiedelten heterotrophen Bakterien.

Braune Hüllen, die rund bis oval erscheinen, finden sich häufig in Kolonien an der Karbonatoberfläche. In Zwickeln und an Grenzen intakter Gloeocapsa-Kolonien bilden diese Hüllen perlschnurartige Spuren (Taf. 5/A), welche im oberen Teil des Gloeocapsa-Biofilms zurückgehen. Die Größe einzelner Gloeocapsa-Zellen beträgt bis zu 4,5 x 5,5 $\mu \mathrm{m}$. Die Maße der braunen Strukturen betragen 2,4 x $6 \mu \mathrm{m}$. Eine genaue Zuordnung ist nicht möglich. Es ist allerdings zu vermuten, dass es sich bei den Hüllen um abgestorbene Gloeocapsa- oder Entophysalis-Zellen handelt.

Die weißen Oberflächen im Topbereich der Stromatolithe (Spritzwasserbereich, Zone I, Taf. 1/B) sind Phormidium-Diatomeen-dominiert (Taf. 6/A, 6/C). Im darunter gebildeten Karbonat (oberer Teil Mikrobialith-Typ 4) finden sich Scytonema und Entophysalis (vgl. Taf. 2/C-G). Die Phormidium-Filamente haben einen Durchmesser von $7 \mu \mathrm{m}$ (Trichome: 3-4 $\mu \mathrm{m}$; äußere Scheiden: 1,2-1,6 $\mu \mathrm{m}$ ) und bilden ein Netzwerk, in dem Diatomeen-Schalen und nicht näher bestimmbare kokkale Strukturen mit einem Durchmesser von 6-8 $\mu \mathrm{m}$ gebunden sind.

Im basalen Karbonat der grün-schwarzen Biofilm-Oberflächen (Zone II) sind Relikte kokkaler Cyanobakterien eingeschlossen, die einen hellen, fluoreszierenden Kern (2-3 $\mu \mathrm{m}$ Durchmesser) und eine äußere, dunkelbraune bis schwarze Hülle (0,9-2,4 $\mu \mathrm{m}$ Dicke) aufweisen. Diese Strukturen haben einen Gesamtdurchmesser von max. 5,4 $\mu \mathrm{m}$ und ähneln 
stark den kokkalen Relikten in den „Entophysalis-Lamellen“ der subfossilen Stromatolithe (Mikrobialith-Typ 4). Allerdings zeigen sich unter UV-Anregung häufig HalbmondStrukturen der Zentren, welche als Gloeocapsa-Teilungsmuster zu interpretieren sind (Taf. 6/B). Rezente Verkalkungsfronten zeichnen die Umrisse von Gloeocapsa-Zellen nach (Taf. 5/C-D).

Die orange-farbenen Oberflächen des Saums um die Bioherm-Basis (Zone III) sowie des Karbonatsaums entlang des gesamten Seeufers repräsentieren Diatomeen-Phormidium-reiche Biofilme. Am Kontakt zum basalen Karbonat (ungleichförmiger, krümeliger Aragonit) mit eingebetteten Cyanobakterien (Taf. 5/E-F) sind die sonst unverkalkten Phormidium-Filamente von einer unregelmäßigen Karbonatzementhülle (Durchmesser 5-9 $\mu \mathrm{m}$ ) umgeben (Taf. 6/D). Ähnlich den Verkalkungsprozessen innerhalb der grün-schwarz gefärbten Biofilmoberflächen sind rezente Präzipitate an Cyanobakterien-EPS gebunden, wodurch sich Abbilder der Kolonie-Umrisse ergeben (Taf. 5/G-H). Neben den elliptischen Diatomeen (Ordnung Pennales, ca. 60 x $18 \mu \mathrm{m}$ ) mit charakteristischen bilateralsymmetrischen Strukturen (Taf. 4/F) kommen Diatomeen der Ordnung Centrales mit einer max. Größe von 10 x $20 \mu \mathrm{m}$ vor (Taf. 6/C).

Schwefelpurpurbakterien stellen den Hauptbestandteil der „flocculent mat“ (GREY et al., 1990) aus dem Seebecken dar. Die Hauptmasse der Organik ist rosa-violett gefärbt. GREY et al. (1990) beschreiben Thyocystis/Thiocapsa als Schwefel enthaltende (Schwefelglobuli) Schwefelpurpurbakterien.

\subsection{Lake Clifton}

\section{Regenzeit}

Rezente Biofilmgemeinschaften werden von Diatomeen und Gloeocapsa sp. dominiert (Taf. 8/A-C) und bilden auf den Thrombolith-Oberflächen bis zu $1 \mathrm{~mm}$ dicke, schleimige Überzüge, die eine orangene Färbung aufweisen, welche in tieferen Bereichen in eine grünschwarze Färbung übergeht. In dichten Biofilmen sind die Cyanobakterien unverkalkt. An der Karbonatbasis und im Hohlraumgefüge der mikrokristallinen Aragonitpräzipitate kommen mit Karbonat umkrustete Phormidium-Filamentspuren vor, deren Durchmesser zwischen $8 \mu \mathrm{m}$ und $13 \mu \mathrm{m}$ betragen (Taf. 8/D). An Diatomeen dominieren elliptische Formen der Ordnung Pennales (Taf. 8/C). Formen der Ordnung Centrales sind selten (Durchmesser 14,5 $\mu \mathrm{m})$. 


\section{Trockenzeit}

Die Diatomeen-Dominanz gegenüber den Proben von 1998 ändert sich nicht, jedoch treten Formen von Centrales häufiger auf (Taf. 7/G, 10/D). Kolonien kokkaler Cyanobakterien (vielfach Gloeocapsa sp. und nicht näher bestimmbare Formen) kommen seltener vor und werden von Filamenten der Cyanobakterien Phormidium sp. und untergeordnet ScytonemaMorphotyp (mit deutlich dickerer Scheide, Taf. 10/C) eingeschlossen. Innerhalb der Biofilme zeigen sich Präzipitate in Form unregelmäßiger Aragonitklumpen (Taf. 10/G-H), welche nicht an exopolymerreiche Substanzen der Cyanobakterien-Scheiden gekoppelt sind. Vergleichbare „thrombolite clots“ sind ebenfalls in der amorphen Mg-Si-Phase eingebettet (Taf. 10/E-F).

Generell sind die rezenten Cyanobakterien unkalzifiziert (Taf. 7/H, 10/C, 10/G). Von unregelmäßigem, z. T. verdicktem Karbonat umgebene Phormidium-Filamente kommen jedoch am Kontakt zum basalen Karbonat (krümelige Aragonite und ,thrombolite clots“) und in dessen Hohlräumen vor (Taf. 10/A). Die zementierten Filamentspuren werden als Ausgangstrukturen der krümelig-klumpigen bis mikrokristallinen Aragonite angesehen. Sehr feine Filamentspuren, reliktische Trichome und kokkale Strukturen sind innerhalb der Mg-SiPhase zu erkennen (Taf. 10/B).

Junge, karbonatische Fällungsprodukte werden einem Stadium vor dem Einsetzen der Mg-SiFällungen zugeordnet. Die Bildung der amorphen Phase zur Zeit der Probennahme wird als (vorerst) letzte Fällungsphase angesehen.

Radialstrahlige Präzipitate sind Bildungen innerhalb des weißen Diatomeen-Biofilms an den Holzpfosten des Steges (Taf. 7/E). Grünalgen-Büschel des Supralitorals sind von KarbonatKrusten umhüllt (Taf. 7/F).

Ältere sowie eigene Untersuchungen an den fossilen Thrombolithen belegen eine Verbreitung von Scytonema sp. und Phormidium sp. (Taf. 6/D) (MOORE, 1983). Weitere Formen filamentöser Cyanobakterien wie Oscillatoria, Dichotrix, Chroococcus, Johannesbaptista, Spirulina und Gomphosphaeria (MOORE \& BURNE, 1994) konnten nicht nachgewiesen werden.

\subsection{Lake Preston}

Rezente Biofilme fossiler Caliche-Krusten sind endolithischer Natur (Taf. 11/A-C). Supralitorale Karbonatschlämme werden von ungleichkörnigen und unlithifizierten Karbonatpartikeln in organikreicher Matrix gebildet und liefern keine Hinweise auf cyanobakterielle Beteiligung (Taf. 11/G), wie sie von fossilen Mikrobialith-Proben belegt sind (Taf. 11/D, 11/F). 


\subsection{Lake Walyungup}

\section{Trockenzeit}

Senkrecht zur Oberfläche der weißen Krusten im Sub- bis Interlitorals sind DiatomeenSchalen angeordnet. Filamentöse Cyanobakterien (Scytonema sp.) sind in Form büschelartiger Strukturen innerhalb des Hydromagnesits vertreten (Taf. 11/A-F). In mikrokristallinem Aragonit eingeschlossene Scytonema-Filamente sind gut erhalten und haben eine gelbliche Färbung. Vollständig erhaltene, aber auch abgerissene Filamente finden sich im Hydromagnesit. Häufig sind unverkalkte Cyanobakterien-Filamente (Taf. 12/F), die teilweise deutliche Trichome $(4 \mu \mathrm{m})$ und dicke Scheiden (max. Gesamtdurchmesser $17 \mu \mathrm{m}$; Scytonema-Morphotyp, Taf. 12/G-H) zeigen. Aragonit-Tuben, wie in CosHELL et al. (1998) beschrieben, konnten nicht gefunden werden. Angeschwemmte Algen (Taf. 12/C) zeigen keine Verkalkungen.

\subsection{Shark Bay}

\section{Regenzeit}

Es wurden die knotigen, grünlichen Mikrobialith-Aufwüchse (Taf. 14/B-C) an den Seiten der subfossilen, agglutinierten Stromatolithe von Hamelin Pool auf rezente Verkalkungsmuster hin untersucht. Diese thrombolithischen Karbonate werden von unregelmäßigen KarbonatKlumpen in einer Matrix aus hauptsächlich Entophysalis-Kolonien und filamentösen Cyanobakterien aufgebaut (Taf. 14/D-F). An Oberflächen und in Hohlräumen sind Entophysalis-Kolonien häufig. Im Kontakt von Meerwasser und Entophysalis bilden sich dünne Karbonatsäume (Taf. 15/A, 15/E-F). Die unkalzifizierte Entophysalis sp. zeigt unter UV-Anregung eine hellblaue Fluoreszenz (Taf. 15/G-H).

Endolithische Aktivität und porenreiche Karbonate sind durch Proben von 1998 belegt (Taf. $16 / G)$, allerdings auf die fossilen und agglutinierten Stromatolithe beschränkt. Endolithen werden von REID et al. (2003) als Solentia sp. interpretiert, wie sie von BahamasStromatolithen bekannt sind (MACINTYRE et al., 2000).

\section{Trockenzeit}

Hamelin Pool-Proben vom April 2002 belegen Karbonatpräzipitate im Kontakt zu Cyanobakterien, welche eine Fluoreszenz bei UV-Anregung zeigen und bräunlich gefärbte Entophysalis-Zellen darstellen (Taf. 15/C-D). Die unregelmäßigen und oberflächenparallelen Präzipitate sind auf die Top-Bereiche der Biofilme beschränkt.

In den äußersten Bereichen der knotigen Anwachsungen von 2002 finden sich keine endolithischen Spuren. 


\section{Mikrofazies}

\subsection{Mikrobialithe, Lake Thetis}

Die Einteilung der Mikrobialithe in Stromatolithe und Thrombolithe erfolgte anhand der makroskopischen Gefüge der Mikrobialithe (RIDING, 1991). Aufgrund vielfältiger Überprägungsmechanismen, welche im lakustrinen Milieu wirksam werden, (vgl. ARP, 1995; FLÜGEL 2004, S. 10, 364) erfolgt - zusätzlich zur Benennung einzelner Strukturmerkmale in Anlehnung an die Klassifikation nach DunHAM (1962), erweitert nach EMBRY \& KLOVAN (1972) - die Einteilung in Mikrobialith-Typen mit interpretativen Zusätzen.

Im Folgenden werden Komponenten, Gefüge und Strukturen beschrieben, die sich in den subfossilen Mikrobialithen des Lake Thetis charakterisieren lassen. Es handelt sich dabei um verhältnismäßig wenige Strukturelemente, wie z.B. Cyanobakterien, Stromatolith-Lamellen, „Organomikrit“-Krusten, sedimentäre Taschenfüllungen, Peloide und eine amorphe Mg-SiPhase. In unterschiedlichen Anteilen sind diese Gefügemerkmale in allen Mikrobialith-Typen vertreten. Eine Abschätzung der Anteile an Gefügemerkmalen, wie z.B. „Organomikrit“Krusten und Cyanobakterien-Mikrofossilien, ist in Abb. 3.1 wiedergegeben.

\subsubsection{Beschreibung der Komponenten}

\subsubsection{Biogene Komponenten}

\section{Cyanobakterien}

\section{Morphotyp Scytonema sp.}

Die Filamente haben einen Durchmesser von 20-25 $\mu \mathrm{m}$ und sind durch Scheinverzweigungen prostrater und erekter Filamentabschnitte charakterisiert.

Die Cyanobakterien sind in Form von gelblich-braunen, nicht-fluoreszierenden Hüllen aus organischen Restsubstanzen der ehemaligen Polysaccharidscheiden, aber auch in Form fadenartiger Resteinschlüsse der eigentlichen Trichome im Zentrum der Hüllen überliefert (Taf. 2/A). Teilweise können Filamente erhalten sein, welche einen Durchmesser von nur $<20 \mu \mathrm{m}$ aufweisen, im Habitus allerdings mit Scytonema sp. identisch sind.

\section{Morphotyp Phormidium sp.}

Die Cyanobakterien-Filamente haben einen Durchmesser von 5-7 $\mu \mathrm{m}$. Sie zeichnen sich durch charakteristisch büscheliges Auffächern der Filamente aus. Im hellgrauen Aragonit sind diese Filamentspuren als hellgraue Strukturen zu erkennen. 


\section{Morphotyp Entophysalis sp.}

In den Stromatolithen kommen gelblich-braune bis braun-schwarze Hüllen organischer Restsubstanzen von 4-5 $\mu \mathrm{m}$ Durchmesser vor. Auffallend ist ein Wechsel von kolonienartigen Anhäufungen der kokkalen Zellrelikte in den tieferen Stromatolith-Bereichen hin zu lagenparallelen „Entophysalis-Lamellen“ in der oberen Stromatolith-Kruste. Durch die bräunlich gefärbten Zellrelikte treten Entophysalis-Lagen bereits makroskopisch deutlich hervor (Taf. 2/A-C). Unter UV-Anregung zeigen die Entophysalis-Lagen eine stark heterogene Autofluoreszenz. Während organische Hüllen nicht fluoreszieren, zeigen die Zentren der ehemaligen Kokken oft eine gelbe Autofluoreszenz. Der umgebende, mikrokristalline Aragonit fluoresziert gelblich-blau, während der feinfaserige Aragonit kaum fluoresziert.

\section{Ostrakoden}

Hauptsächlich sind Ostrakoden als Bestandteil der sedimentären Taschenfüllungen erhalten. Peloidale, mikritische Bereiche enthalten ebenfalls dislozierte, regellose Ostrakoden-Schalen und Schalenbruchstücke. Teilweise können auch gut erhaltene Schalen mit Zement gefüllt sein.

\section{Gastropoden}

Innerhalb der Stromatolithe ist Coxiella sp. in die Aragonite und peloidalen Bereiche eingebettet.

\section{Foraminiferen, Rotalgen und Mollusken}

Foraminiferen, Rotalgen- und Molluskenfragmente sind Bestandteil der Taschenfüllungen und peloidalen Bereiche innerhalb der Mikrobialithe. Sie stammen aus den holozänen Karbonatsanden der Hershell Formation.

\subsubsection{Abiogene Komponenten}

\section{Peloide}

Peloide sind nach FLÜGEL (1982b) mikritische, rundliche Partikel, gewöhnlich ohne Internstruktur, die elliptische bis runde Umrisse aufweisen. Die Bezeichnung ist ein Sammelbegriff für Komponenten unterschiedlicher Genese.

Die Peloide mit Durchmessern von ca. 80-200 $\mu \mathrm{m}$ kommen zum einen innerhalb sedimentärer Taschenfüllungen vor und sind von einem max. $37 \mu \mathrm{m}$ breiten Mikrit-Saum umgeben. Die Taschenfüllungen sind dabei reich an holozänem Schill. Mikrokristalliner, klumpiger Aragonit bildet die Matrix für die häufig dichtgelagerten Peloide. Zudem gibt es Bereiche, in denen die Peloide in Korn-Korn-Kontakt angeordnet sind und in denen eine Matrix fehlt. 
Aufgrund der Assoziation mit Intra- wie auch Extraklasten im gleichen Korngrößenspektrum, sowie der zum Teil geringen bis schlechten Rundung werden die Peloide im Wesentlichen als Erosionsprodukte mikrokristalliner, vermutlich schwach verfestigter Sedimente angesehen.

\section{Intra- und Extraklasten}

$\mathrm{Zu}$ den Intraklasten werden schlecht bis angerundete, mikrokristalline Komponenten gezählt, welche einen Durchmesser größer $220 \mu \mathrm{m}$ aufweisen. Extraklasten kommen in Form umgelagerter, gut bis schlecht gerundeter Siliziklasten, meist Quarz vor, und werden als holozäner Eintrag angesehen. Sie kommen, wie auch die Peloide, sowohl in den Taschenfüllungen als auch vereinzelt innerhalb der Aragonite vor.

\section{Amorphe Mg-Si-Phase}

Die amorphe Phase ist durch alle Mikrobialith-Typen des Lake Thetis verfolgbar. Sie ist hellgelb-orange bis gelb-braun gefärbt, meist klar, selten milchig trübe und zeigt zwischen gekreuzten Polarisatoren undulöse Auslöschung (Taf. 2/F, 2/G). Die Phase ersetzte vermutlich ehemalige Gallertscheiden der Cyanobakterien Scytonema sp., wodurch diese, besonders in oberen Mikrobialith-Typen, sehr gut erhalten sind (Taf. 2/A). Zwischen Aragonit und „Organomikrit“-Krusten tritt die amorphe Phase ebenfalls auf und verläuft lagenparallel, jedoch nicht durchgängig, zu den inneren Lagen der „Organomikrite“ (Taf. 2/E-F). Dabei verläuft der Phasenübergang von Aragonit zu Mg-Si-Phase häufig diffus (Taf. 2/D). Übergänge von Mg-Si-Phase und „Organomikriten“ verlaufen in Form klarer Grenzlinien. Zudem treten klumpenartige Bereiche mit z.T. ausgeprägten Kantenflächen ohne erkennbares kristallographisches Muster innerhalb der Aragonite und auch Taschenfüllungen auf.

\subsubsection{Beschreibung der Gefüge}

\section{Stromatolithische Lamination und Hohlraumgefüge}

Eine stromatolithische Lamination ist besonders in den oberen Bereichen der Stromatolithe des Interlitorals ausgeprägt. In der oberen Kruste findet sich der Wechsel von Cyanobakterien-Lamellen, der im mittleren Teil der Kruste charakteristische SHStromatolith-Säulen aufweist. Diese internen Stromatolithe bauen nach oben orientierte Strukturen mit Aufdomungen auf und enthalten in Vertiefungen sedimentäre Taschenfüllungen mit peloidalem Anteil, aber auch terrigenem Eintrag.

In tiefer liegenden Stromatolithen wird eine Lamination häufig ausschließlich durch horizontal verlaufende Hohlraumgefüge deutlich oder geht vollständig in ein thrombolithisches Makrogefüge über (Abb. 3.1). 


\section{„Organomikrit"-Krusten}

Ehemalige Lösungshohlräume auskleidende, laminierte Krusten werden von REITNER et al. (1996) als „Sinter-ähnliche“-Krusten beschrieben, deren Bildung mit der Nukleation an organischen Matrizen innerhalb der Organik-haltigen Krusten (Autofluoreszenz) in Zusammenhang gebracht wird. Eine rein abiotische Bildung ist auszuschließen (REITNER et al., 1996). Die an nicht lebende, organische Substanzen gekoppelte Mineralisierung führt zu einer autochthonen Mikrit-Produktion (“Automikrit“, NEUWEILER et al., 1999).

Der Begriff „Organomikrit“ wurde für die mikrokristallinen Aragonitanteile der Krusten gewählt - entgegen der ursprünglichen Definition für Mikrit als Abkürzung für „microcrystalline calcite“ durch FOLK (1959) - um die mikrokristalline, homogene Struktur der aragonitischen Krusten zu verdeutlichen.

„Organomikrit“-Krusten weisen eine scharfe Lamination auf. Einzelne Lagen werden teilweise durch Zementzwischenlagen getrennt. Die Krusten kommen unregelmäßig verteilt innerhalb der klumpigen Aragonite und peloidalen Bereiche vor. Teilweise trennen die „Organomikrite“ diese beiden Phasen auch voneinander. „Organomikrite“ enthalten keine Partikel. Ehemalige Lösungshohlräume sind mit „Organomikrit“-Krusten ausgekleidet, kleine ehemalige Hohlräume sind vollständig mit „Organomikrit“ gefüllt.

Feine Klüfte und Hohlräume, welche vertikal innerhalb einer Stromatolith-Kruste, aber auch horizontal als Trennung zweier Stromatolith-Typen auftreten, sind ebenfalls mit „Organomikrit“-Krusten häufig reliefausgleichend überzogen (Taf. 2/H).

Einige Bioherme mit knotiger Oberfläche zeigen eine weiße Top-Lage, welche von reliefausgleichenden „Organomikriten“ aufgebaut wird und eine maximale Dicke von 2 mm erreicht.

\section{Sedimentäre Taschenfüllungen}

Die sedimentären Taschenfüllungen werden häufig durch Säume von „Organomikrit“ von dem umliegenden Aragonit getrennt und stellen die Füllung ehemaliger konkaver Einbuchtungen dar. Die enthaltenen Komponenten sind Peloide, Intraklasten, Siliziklasten und holozäner Schill (Foraminiferen, Rotalgen- und Molluskenfragmente). Untergeordnet ist die amorphe $\mathrm{Mg}$-Si-Phase vertreten. 


\subsubsection{Beschreibung der Mikrobialith-Typen}

\section{Mikrobialith-Typ 1a: Peloidale Thrombolithe}

Beschreibung: Mit diesem Typ werden thrombolithische Karbonate zusammengefasst. Zum einen besteht deren Gefüge aus Aragonitzementen, welche durch dünne „Organomikrit“Krusten von sogenannten Taschenfüllungen abgetrennt sind. Die Taschenfüllungen nehmen ein Gesteinsvolumen von ca. 50\% ein und bestehen aus Peloid-Packstones mit dunkelbraunen Peloiden mit einer krümeligen bis mikrosparitischen, dunkelgrauen Matrix. Die Peloide und zusätzlich enthaltenen Intraklasten sind teilweise schlecht gerundet. Holozänes Material kommt nur sehr untergeordnet in den Taschenfüllungen vor. Cyanobakterien-Filamente kommen häufig, jedoch nur in Form reliktischer Spuren im Aragonit vor, Scytonema sp. in Form von silifizierten Filamenten, Phormidium sp. und unbestimmbare Cyanobakterienreste in Form von reliktischen Spuren im klumpigen Aragonit. Die „Organomikrit“-Krusten zeigen nur sehr wenige dünne Zementzwischenlagen.

Zudem kann dieser Mikrobialith-Typ aus ebenfalls klumpigen bis krümeligen Aragoniten bestehen und sehr viele silifizierte Scytonema-Filamente enthalten. Die Scytonema-Filamente treten sowohl im horizontalen wie vertikalen Anschnitt auf und sind selten orientiert.

Interpretation: Der Mikrobialith-Typ entstammt dem steileren Hang am N-Ufers, wo die Wellenaktivität vergleichsweise hoch ist (vgl. GREY et al., 1990). Die stark angewitterten Thrombolithe sind während des Seespiegeltiefstandes exponiert. Sie lassen keine aktive Karbonatfällung erkennen und haben unregelmäßige, weißlich gefärbte Oberflächen. Dieser Mikrobialith-Typ wird einer älteren Generation des Sub- bis Interlitorals zugeordnet. Die krümeligen Strukturen innerhalb der Aragonite sind teilweise zu Kolonien angeordnet und werden als Entophysalis-Relikte interpretiert. Die Taschenfüllungen deuten auf ein Bewegtwassermilieu im Sublitoral hin. Die Lagerungsverhältnisse der Cyanobakterien deuten ebenfalls auf Verhältnisse hin, die zu einer Umlagerung der wenig bis nicht lithifizierten Scytonema-dominierten Biofilme geführt haben.

\section{Mikrobialith-Typ 1b: Thrombolithe}

Beschreibung: Dieser Mikrobialith-Typ zeichnet sich durch lagig angeordnete Hohlraumgefüge aus, welche bis zu $4 \mathrm{~cm}$ breit und $5 \mathrm{~mm}$ hoch werden können. Diese Hohlräume werden von „Organomikrit“-Krusten ausgekleidet, deren Anteil an Zementzwischenlagen sehr gering ist. Cyanobakterielle Spuren sind in Form von undeutlichen kokkalen wie auch filamentösen Gefügen im klumpigen bis zementreichen Aragonit erhalten. 
Die fibrösen Zemente der Hohlräume gehen als letzte Phase in nadelige Zemente über. Eine einschlussreiche Mg-Si-Phase erscheint dunkelgelb, klare Mg-Si-Phasen erscheinen hellorange bis gelb. Vereinzelt gibt es zementgefüllte Ostrakoden-Schalen. Durch „Organomikrit“ abgegrenzte Bereiche bestehen aus Peloid-Packstones, deren Peloide mikritische Säume aufweisen und in mikritischer bis mikrosparitischer Matrix eingebettet sind. Auffallend ist, dass Scytonema-Filamente, wie auch Phormidium sp. und eindeutige Belege für Entophysalis sp., fehlen.

Interpretation: Dieser Typ stammt ebenfalls von den stark angewitterten Karbonat-Terrassen des N-Ufers und des E-Ufers. Uferparallele Karbonatsäume zeigen stark angewitterte, hellgraue Karbonatkörper (Taf. 1/F), die z.T. einen übergeordneten konzentrischen Aufbau erkennen lassen. Im Handstück findet sich allerdings ausschließlich ein thrombolithisches Gefüge, weshalb von einer Benennung als Stromatolith abgesehen wird. Entsprechend dem Mikrobialith-Typ 1a ist der Typ $1 \mathrm{~b}$ ebenfalls einer älteren Mikrobialith-Generation zuzuordnen.

\section{Mikrobialith-Typ 2a: Intra- und Extraklast-reiche Thrombolithe}

Beschreibung: Unterhalb von Mikrobialith-Typ 2b befindet sich ein Bereich aufgedomter „Karbonatkissen“, deren Oberflächen keine weißen oder knotigen Krusten bilden, sondern durch einen unverfestigten Karbonatsand aus Ostrakoden-Schalen und holozänem Material von den darüber liegenden „Kissen“ getrennt sind. Diese ca. 14 x 11 cm großen „Kissen“ werden maximal 7,5 cm mächtig.

Mikroskopisch ist für den Mikrobialith-Typ 2a ein krümeliger Aragonit charakteristisch, der allochtone, holozäne Komponenten (Pack-Grainstones) enthält und dessen Hohlräume von „Organomikrit“- und Zementlagen ausgekleidet werden. Offene Hohlräume sind zum Teil gefüllt mit matrixfreien Pack-Grainstones, die aus Ostrakoden-Schalenbruch, holozänen Komponenten und schlecht gerundeten Peloiden bestehen. Filamente des Cyanobakteriums Scytonema sp. kommen nicht vor. Schemenhaft sind häufig ehemalige Filamentspuren zu erkennen, die eventuell dem Morphotyp Phormidium zugeordnet werden können. Die amorphe Mg-Si-Phase ist vereinzelt, jedoch nicht lagenweise vertreten. „Organomikrit“Krusten mit reliefausgleichenden Zementlagen kleiden Hohlräume aus. Die das Gestein als äußere Krusten umschließenden, dunkelbraunen „Organomikrite“ weisen nur einen geringen Anteil an Zementzwischenlagen auf. Die letzte Zementphase ist ein nadeliger Aragonitzement, der die Hohlräume jedoch nicht immer vollständig füllt. 
Vorkommen: Dieser Mikrobialith-Typ entstammt der supra- bis interlitoralen Zone am SUfer und bildet den tieferen Teil der zweiphasig aufgebauten Bioherm-Dome. Zonen mit kolonial angeordneten Mikritklumpen werden als Hinweis auf Entophysalis sp. gewertet.

\section{Mikrobialith-Typ 2b: Meteorisch-geprägte, Intra- und Extraklast-reiche Thrombolithe}

Beschreibung: Die Bioherme bestehen aus dichtgepackten, polygonalen „Kissen“, die an der Oberfläche eine weiße, glatte Kruste bilden, welche zu den Rändern hin in eine knotige, dunkel-graue Kruste übergeht (Taf. 1/D). Die „Kissen“ werden maximal $13 \mathrm{~cm}$ hoch und haben Abmessungen von ca. 7 x $10 \mathrm{~cm}$. Im Querschnitt zeigen sich lentikuläre Hohlräume, die eine untergeordnet stromatolithische Struktur nachzeichnen.

Im Mikroskopischen wird ein krümeliger Aragonit deutlich, der durch „Organomikrit“Krusten und Zementzwischenlagen von Peloid-Packstones abgetrennt ist. Innerhalb der klumpigen, mikrokristallinen bis mikrosparitischen Aragonite bestehen allerdings auch Peloid-Packstone-Bereiche, welche nicht durch „Organomikrit“-Krusten abgetrennt sind. Die Peloid-Packstones enthalten einen hohen Anteil an holozänen Extraklasten. Die Peloide und der holozäne Schill zeigen mikritische Umrandungen und liegen in einer mikrokristallinen Matrix, welche in manchen Bereichen ausgewaschen, aber auch mikrosparitisch ersetzt sein kann. Cyanobakterielle Filamentspuren treten nicht auf. Einzig kolonienähnliche Peloide ähneln Entophysalis. Die „Organomikrit“-Krusten weisen ausgeprägtere und breitere Zementzwischenlagen als im Mikrobialith-Typ 2a auf. Die Zementzwischenlagen sind zudem häufig getrübt, radialfibrös und reliefausgleichend ausgebildet, wodurch sie in Vertiefungen breiter sind. Die oberste Lage wird von einer reliefausgleichenden, feinlaminierten „Organomikrit“-Kruste, im Wechsel mit Zementlagen, gebildet, wie sie auch die jungen, organikreichen Stromatolithe (Mikrobialith-Typ 4) zeigen. Darunter befindet sich ebenfalls eine „Organomikrit“-Kruste, welche allerdings kaum Zement als Zwischenlagen enthält. Zwischen diesen beiden „Organomikrit“-Krusten befindet sich eine Zone mit gelb-bräunlich gefärbten Cyanobakterien-Filamenten, welche Scytonema sp. ähneln, im Durchmesser allerdings etwas kleiner sind. Die Mg-Si-Phase füllt ehemalige Hohlräume im Kontakt zwischen „Organomikrit“ und klumpigem Aragonit und Peloid-Packstones.

Interpretation: Die exponierten Thrombolithe entstammen dem interlitoralen Bereich des SUfers und liegen bis $\mathrm{zu} 6 \mathrm{~m}$ vom saisonal überfluteten Ufersaum entfernt. Die „Organomikrite“ mit Zementzwischenlagen gehen auf einen kontinuierlichen Wechsel von Emersions- und Überflutungsphasen zurück. Eventuell geht die mit Karbonatsand gefüllte 
Trennungsfläche zwischen den Mikrobialith-Typen 2a und 2b auf ein Emersionsereignis zurück.

\section{Mikrobialith-Typ 3: Meteorisch-geprägte Stromatolithe}

Beschreibung: Die Bioherme kommen vereinzelt oder in Form seitlich zusammengewachsener Stromatolithe vor (Taf. 1/A). Sie sitzen dem zum Seebecken leicht absinkenden Karbonatsaum (,consolidated platform carbonates“, GREY et al., 1990) auf, wodurch marginal liegende Stromatolithe höher aus dem Wasserspiegel herausragen als proximale. Zwischen einem zentralen Bioherm-Bereich, welcher aus verfestigtem Karbonatsand besteht, und diesem Mikrobialith-Typ befindet sich ein verwitterungsanfälliger Hohlraum, der Reste von Karbonatsand enthält.

Die interlitoralen Bioherme werden von zwei Stromatolith-Krusten aufgebaut. Die untere Kruste (Mikrobialith-Typ 3) ist max. $6 \mathrm{~cm}$ hoch und weist an vertikalen Kluftflächen und am Top „Organomikrit“-Ü̈berzüge auf.

Mikroskopisch weist dieser Stromatolith-Typ, neben klumpigem Mikrit und feinen „Organomikrit“-Lagen, einen hohen Anteil an fibrösem Aragonitzement auf (Taf. 2/H). Durch horizontal verlaufende Hohlraumgefüge, die mit „Organomikrit“-Krusten ausgekleidet sind, entsteht ein makroskopisch deutlich stromatolithisches Gefüge. Mehrfach umkrustete Peloide und holozäner Schill bilden in einer mikritischen Matrix Zonen von PackstoneTaschenfüllungen, die unregelmäßig an „Organomikrit“-Krusten, Zemente oder Mikrit anschließen. Die Breiten der dunklen und hellen Lagen der „Organomikrit“-Krusten variieren sehr stark. Zum Zentrum der Lösungshohlräume werden die Zementlagen zunehmend dünner, die mikrokristallinen dunkelbraunen Lagen der „Organomikrite“ erscheinen dadurch dicker und werden bis $\mathrm{zu} 300 \mu \mathrm{m}$ breit. Lagenweise sind Spuren erekter, paralleler oder buschförmiger und silifizierter Scytonema-Scheiden im oberen Teil häufig. EntophysalisKolonien sind in Form klumpig-krümeligen, dunkelgrauen Aragonites enthalten. Bereiche innerhalb der Aragonite sind mit orangefarbener Mg-Si-Phase imprägniert. Phormidium sp. ist in Form büschelartiger Strukturen innerhalb des krümeligen Aragonites zu erkennen. Ehemalige Lösungshohlräume bilden etwa $60 \%$ des Gesteinvolumens. Sie sind von bis zu $2 \mathrm{~mm}$ dicken „Organomikrit“-Krusten ausgekleidet, welche auch die Außenflächen überziehen. Diese reliefausgleichenden Krusten setzen sich aus faserigen Aragonitzementlagen (nicht fluoreszierend) und bräunlichgrauen Mikritlagen mit starker Autofluoreszenz („Organomikrit“) zusammen. Nadelige Zemente treten sporadisch in offenen Hohlräumen auf. 


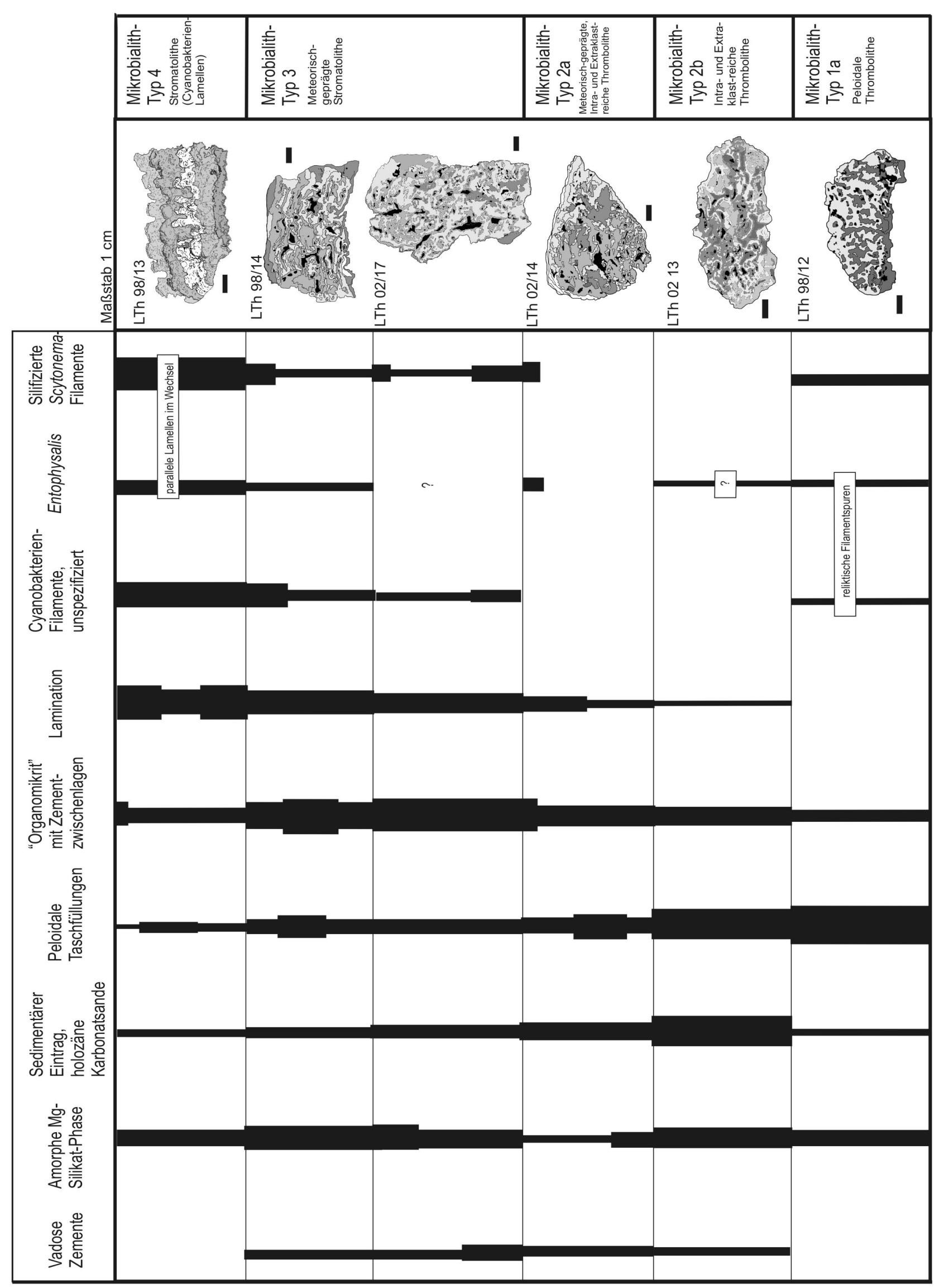

Abb. 3.1: Vergleichende Abschätzung der Anteile an Cyanobakterien-Filamenten, “Organomikrit"-Krusten, amorpher Mg-Si-Phase, terrigenen Taschenfüllungen in den Mikrobialith-Typen aus inter- bis supralitoralen Bereichen des Lake Thetis. 
Interpretation: Dieser Mikrobialith-Typ wird als mittlere Stromatolith-Kruste interpretiert, welche in den Biohermen am S-Ufers in direktem Kontakt zu der darüber liegenden Kruste (Mikrobialith-Typ 4) vertreten ist. Die faserigen, radialfibrösen Zemente (Zementzwischenlagen der „Organomikrit“-Krusten) und die z.T. nadeligen Zemente werden als Hinweis auf meteorisch-vadose Verhältnisse gewertet, welche aus episodischen Expositionen hervorgehen.

Trotz hohem Silikatanteil konnte aufgrund der röntgenamorphen Struktur keine Mg-Si-Phase durch RDA-Untersuchungen nachgewiesen werden. Allein die aragonitsche Phase (kein MgKalzit) ist röntgenographisch nachweisbar (vgl. Kap. 5.1).

Der die Bioherme unterlagernde Karbonatsaum umfasst einen 1-8 m breiten Bereich entlang des gesamten Seeufers (Sublitoral). Eine zeitliche Zuordnung war nicht möglich, zumal am $\mathrm{N}$ - und E-Ufer die thrombolithischen Terrassen direkt in den Karbonatsaum übergehen.

\section{Mikrobialith-Typ 4: Stromatolithe (Cyanobakterien-Lamellen)}

Beschreibung: Die knotigen Oberflächen der Stromatolithe sind im oberen Teil grau-weiß und gehen dann in eine grüne und orange Färbung über. Unterschiedliche Mikrobenmatten bedingen diesen farblichen Wechsel, der als makroskopisches Kriterium für die Einteilung in Zonen herangezogen wurde (Zonen I bis III). Die Bioherme weisen einen kranzförmigen Saum an der Bioherm-Basis auf, dessen Oberkante den Seespiegeltiefstand markiert (Taf. 1/B). Die Dächer der marginalen und nur teilweise vom Wasser umgebenen Bioherme sind erodiert und eingebrochen, wodurch sich ein mehrphasiger Aufbau zeigt (Taf. 1/D). Wasserbedeckte oder in einer Tiefe von bis zu $15 \mathrm{~cm}$ liegende Stromatolith-Köpfe weisen geschlossene Dächer auf. Marginale wie auch proximale Stromatolithe werden von der SPBreichen ,flocculent mat“" umspült (Taf. 1/C).

Die Stromatolithe werden von jeweils 2-5 mm dicken Lamellen aufgebaut (Taf. 1/G). Dabei können grundsätzlich drei Lamellentypen entsprechend ihrer dominierenden CyanobakterienMorphotypen unterschieden werden:

„Phormidium-Lamellen“ werden von feinfaserigem bis grobfaserigem Aragonit gebildet, welcher von erekten, pinselartig angeordneten Spuren dünner Cyanobakterien-Filamente (Typ Phormidium, Filamentdurchmesser 5-7 $\mu \mathrm{m}$ ) durchzogen wird.

„Scytonema-Lamellen“ werden ebenso von Aragonit aufgebaut (Taf. 2/A). Sie sind jedoch von deutlich dickeren Filamenten (Durchmesser 20-25 $\mu \mathrm{m}$ ) durchzogen, welche durch Scheinverzweigungen und einem Wechsel prostrater und erekter Filamentabschnitte charakterisiert sind (Taf. 2/A). Die Cyanobakterien sind dabei in Form von gelblich-braunen, 
nicht-fluoreszierenden Hüllen aus organischen Restsubstanzen der ehemaligen Polysaccharidscheiden überliefert. Vereinzelt sind auch fadenartige Resteinschlüsse der eigentlichen Trichome im Zentrum der Hüllen erkennbar (Taf. 2/A). Im mittleren Teil der Stromatolith-Kruste werden lagenparallele Lamellen von aufgedomten Stromatolith-Säulen abgelöst. Sedimentäre Taschenfüllungen füllen Vertiefungen oder sekundäre Lösungshohlräume in diesem Bereich aus.

Phormidium- und Scytonema-Morphotypen kommen vergesellschaftet in den Lamellen vor. In den älteren beiden Stromatolith-Phasen ist zudem oft ein hoher Anteil an allochthonen Karbonatpartikeln, umgelagert aus den umgebenden holozänen marinen Karbonaten, zu beobachten (Foraminiferen, Rotalgen- und Molluskenfragmente).

„Entophysalis-Lamellen“ bestehen aus mikrokristallinem Aragonit, welcher von zahlreichen kokkalen Zellrelikten durchsetzt ist (Taf. 2/A-C). Letztere sind gelblichbraune bis braunschwarze Hüllen organischer Restsubstanzen und von 4-5 $\mu \mathrm{m}$ Durchmesser. Durch die Zellrelikte sind Entophysalis-Lagen bereits makroskopisch an ihrer bräunlichen Färbung zu erkennen (Taf. 1/G, 2/A, 2/C). Unter UV-Anregung zeigen die Entophysalis-Lagen eine stark heterogene Autofluoreszenz. Während organische Hüllen nicht fluoreszieren, zeigen die Zentren der ehemaligen Kokken oft eine gelbe Autofluoreszenz. Der umgebende mikrokristalline Aragonit fluoresziert gelblich-blau, während der feinfaserige Aragonit kaum fluoresziert.

Lösungshohlräume bilden etwa 50\% des Gesteinvolumens. Sie sind von bis zu 1,5 mm dicken „Organomikrit“-Krusten ausgekleidet, welche auch die Außenflächen der Stromatolithe überziehen. Oberflächennah sind Stromatolith-Lamellen, insbesondere faserige Aragonitlagen, von endolithischen Filamenten mit 2-3 $\mu \mathrm{m}$ Durchmesser durchzogen. Dimensionen und Verzweigungsmuster deuten auf Pilze als Verursacher hin.

Interpretation: Bereits GREY et al. (1990) beschreiben hellbraune, amorphe Partikel in den SPB-reichen Sedimenten des Seebeckens. Bisher nicht bekannt waren Mg-reiche Silifizierungen, welche in den primären Stromatolith-Lamellen verbreitet sind. Die Silifizierung geht dabei meist von Lösungshohlräumen bzw. dem Kontakt zu „Organomikrit“Krusten aus. Zudem sind Scytonema-Filamente durch silifizierte Gallert-Scheiden sehr gut erhalten. Elektronenstrahlmikrosonden-Daten belegen ein $\mathrm{Mg} / \mathrm{Si}$-Molverhältnis von $1: 1,1$ und 1 : 1,25 (vgl. Kap. 5.3), welches nahezu dem Mg/Si-Verhältnis der amorphen Mg-SiPhase der Mikrobialithe im Satonda Kratersee entspricht (Mg/Si-Verhältnis $1: 1$; ARP et al., 2003; 2004). Wie diese zeigen die Silifizierungen in den Lake Thetis-Stromatolithen unter UV-Anregung eine deutliche, gelbe Autofluoreszenz. 
Die säulenartige Form der einzelnen Lagen, besonders im Bereich der ScytonemaPhormidium-Zone (mittlerer Teil des Handstücks) werden auch als modernes Analog für präkambrische Stromatolithe angesehen (GREY et al., 1990). Die Autoren beschreiben für diesen Typus eine Zone geringer Wellenaktivität sowie eine hohe Produktion an Mucilage des „,nodular“ Biofilms, wodurch der wachsende Stromatolith mit den Säulen geschützt wird.

Die tieferen Teile der Mikrobialithe haben ein Alter zwischen 1210 und 1080 Jahren, das Alter der äußeren Kruste wird mit $90( \pm 240)$ bis $5( \pm 0,4)$ Jahren angegeben (GREY et al., 1990). Eine exakte Zuordnung dieser äußeren Kruste zu den Mikrobialith-Typen 3 und 4 ist nicht möglich.

\section{Caliche}

Beschreibung: Die Karbonatkruste zeichnet sich makroskopisch durch eine dunkelbraune Färbung, eine hohe Porosität, durch bis zu 0,5 mm große, hellgraue, weiße Extraklasten (Schalenbruch von Mollusken, Rotalgen- und Echinodermen-Fragmente, Foraminiferen, terrigene Siliziklasten) sowie durch graue, mehrfach aufgearbeitete Peloide aus. Die Extraklasten, wie auch schlecht bis gut gerundete Peloide, sind von ungleichmäßig diffusen, braunen Mikritsäumen umgeben, welche bei dichtgelagerten Komponenten ineinander übergehen. Die hohe Porosität entsteht durch nicht zementierte Zwickel zwischen den Komponenten.

Interpretation: Die Karbonatkruste ist ein holozäner, mariner Karbonatsand, dessen Komponenten über vadose Mikritbrücken zementiert sind. Er wird als Caliche interpretiert. Die Probe stammt von der südlich gelegenen Überschwemmungsebene.

\subsubsection{Zusammenfassende Interpretation}

Die Mikrobialith-Generationen, welche allesamt in der photischen Zone des Sub- bis Interlitorals entstanden sind, zeigen eine Verlagerung des Seeufers in Richtung Seemitte. Eine Absenkung des Seespiegels im Laufe der Seegeschichte und ein Wechsel von marinen zu lagunären und letztlich lakustrinen Verhältnissen (vgl. GREY et al., 1990) spiegelt sich in den höheren Anteilen an siliziklastischem Material in älteren Mikrobialithen wider.

In Abhängigkeit zu ihrer lokalen Verbreitung werden die Mikrobialith-Typen 3 Zonen zugeordnet. 
- Zone A: Karbonat-Terrassen des N- und E-Ufers (Supra- bis Interlitoral). Die eingeebneten Mikrobialithe (Typen 1a und 1b) stellen ein altes Bioherm-Vorkommen dar, zu dem auch stark erodierte Bereiche des S-Ufers gezählt werden.

- Zone B: Thrombolithische Bioherme, S-Ufer (Supra- bis Interlitoral). Die Mikrobialith-Typen 2a und 2b werden einer mittleren Mikrobialith-Generation zugeordnet.

- Zone C: Stromatolith-Bioherme, S-Ufer (Interlitoral). Die Top-Bereiche der Mikrobialith-Typen 3 und 4, insbesondere die weißen „Organomikrit“-Krusten, werden als jüngste subfossile Mikrobialith-Bildungen angesehen. Rezente Fällungsprodukte bilden sich allerdings an den Flanken und umsäumen die Basis dieser Bioherme (Kap. 2).

Alle drei Zonen zeigen direkte Übergänge zu dem uferparallelen Karbonatsaum. Eine genau zeitliche Eingliederung nach rein morphologischen Kriterien ist schwer möglich. Einzig das Einsetzen des Bioherm-Wachstums in Zone C markiert eine Obergrenze, da diese Bioherme dem Karbonatsaum aufgewachsen sind.

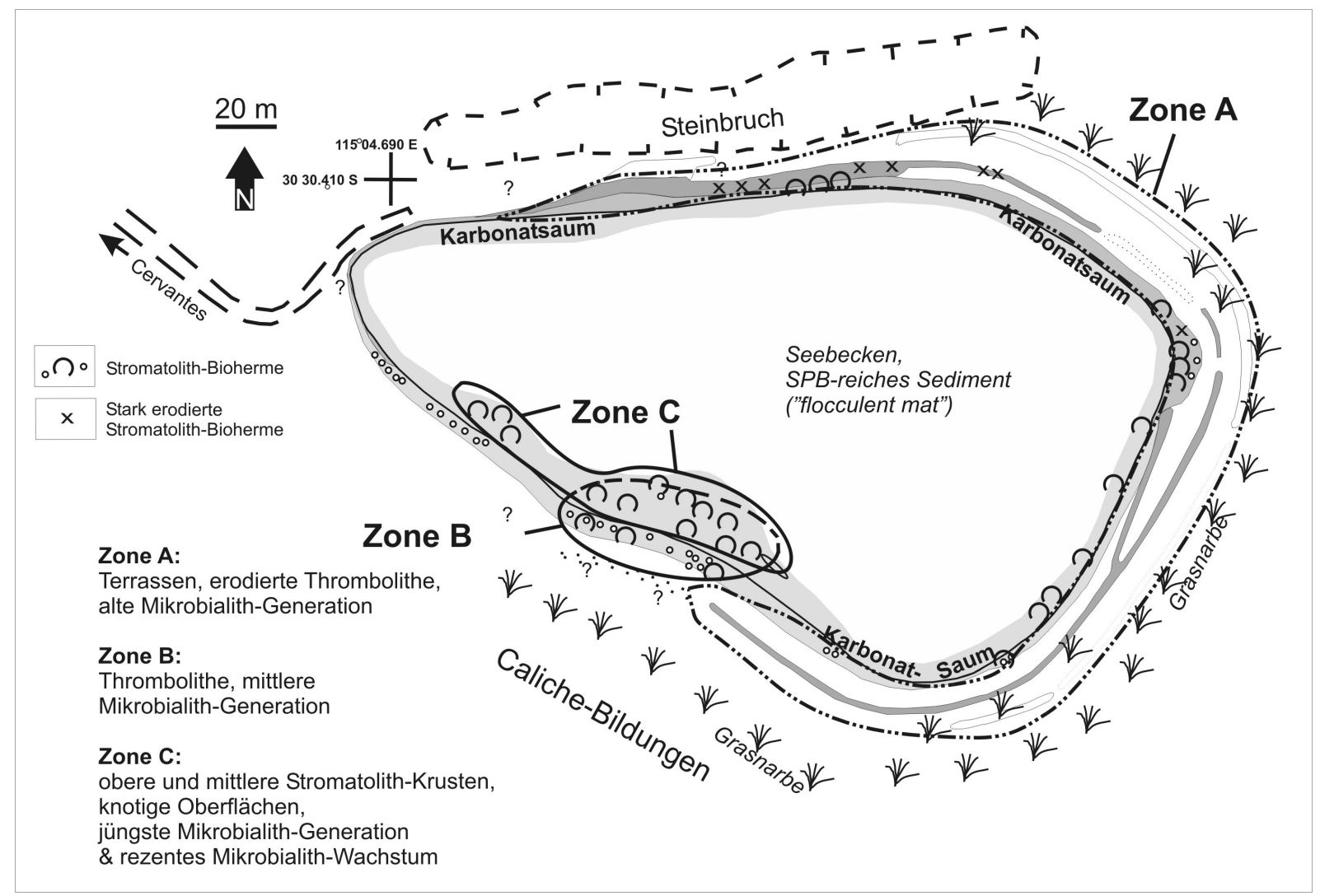

Abb. 3.2: Das Seeufer des Lake Thetis lässt sich anhand der Mikrobialith-Typen in drei Zonen gliedern 


\subsection{Mikrobialithe, Lake Clifton}

Beschreibung: Entlang des E-Ufers befindet sich ein ca. $100 \mathrm{~m}$ breiter Bioherm-Saum (Taf. 1/A). Erosiv stark angegriffene Stromatolithe zeigen einen strikt konzentrischen Aufbau (Taf. 7/B) und gehen im Bereich des Interlitorals in runde bis kegelförmige Stromatolithe über (Taf. 7/C). Letztere zeigen im Sublitoral „,vent“-artige Öffnungen. Mit Karbonatschlamm bedeckte, fossile Stromatolithe ergeben stellenweise Vertiefungen, in denen sich Pfützen (,,ponds“) bilden (Taf. 7/C). Hier finden sich mit Karbonat umkrustete Grünalgen-Büschel.

Subrezente Anteile der stromatolithisch-thrombolithischen Bioherme werden von einem Gerüst aus unregelmäßig-lappigen Karbonat-Klumpen (,thrombolite clots“; Taf. 9/A, 11/G) aufgebaut. Zwei Drittel des Gesteinsvolumens werden von Porenraum eingenommen. Durch das Verteilungsmuster der Porenräume entsteht makroskopisch ein grob stromatolithisches Gefüge im cm-Bereich. Tiefere Bereiche der Thrombolithe (ca. $30 \mathrm{~cm}$ unterhalb der Oberfläche) werden von Mikrit-Klumpen aufgebaut, welche allerdings weniger krümelig, dichter strukturiert und hellgrau gefärbt sind.

Die Thrombolith-Klumpen bestehen aus mikrokristallinem bis feinfaserigem Aragonit (dunkelgrau im Hellfeld), welcher erekte, z.T. radial-büschelförmig angeordnete Cyanobakterien-Filamente der Gattung Scytonema sp. und Phormidium sp. einschließt (Taf. 9/B-C). Die Filamente weisen einen Gesamtdurchmesser bis zu $50 \mu \mathrm{m}$ auf, wobei der Zellfaden bis zu $12 \mu \mathrm{m}$ Durchmesser erreicht. Diese subrezenten Filamente werden von einem faserigen, leicht gelblichen Aragonit zementiert, welcher zentripetal ins Innere der Hohlformen gesprosst ist. Feinkristalliner und dichter zementiert sind die Randzonen der Thrombolith-Klumpen, wo Scytonema sp.- und Phormidium sp.-ähnliche Strukturen in Form von 10-15 $\mu \mathrm{m}$ bzw. 6-9 $\mu \mathrm{m}$ dicken Filamentspuren auftreten. Taschenfüllungen des Thrombolith-Gerüstes zeigen lockeren Karbonatsand aus unregelmäßigen ThrombolithFragmenten (100-500 $\mu \mathrm{m}$ Größe), Gastropoden-Schalen (Coxiella sp.), vereinzelt CharaceenOogonien und kleine Quarzkörner (Taf. 9/D, 11/H). Das von unregelmäßigen Karbonatklumpen und filamentösen Strukturen aufgebaute Karbonatgerüst ist in einer amorphen Mg-Si-Phase eingebettet (Taf. 8/E-H, 9/E-H). Unter UV-Anregung fluoresziert die Si-Phase, wodurch Schrumpfungsrisse verstärkt deutlich werden. Stellenweise zeigen auch die mikritischen Karbonate eine schwächere Fluoreszenz (Taf. 8/F, 8/H, 9/A).

Interpretation: Die Mg-Si-Phase, welche die subrezenten Karbonatklumpen und filamentösen Strukturen umschließt, wird als rezentes, autochthones Präzipitat interpretiert. 
Die Filamentspuren der Cyanobakterien werden dementsprechend als subfossil eingestuft. Insbesondere im Fall von Scytonema sp. bestätigt sich diese Beobachtung (vgl. Kap. 2).

\subsection{Mikrobialithe, Lake Pollard}

Beschreibung: Untersucht wurden trockengefallene, teilweise erodierte und inaktive Bioherme des S-Ufers. Ein Stromatolith-Wachstum findet nicht mehr statt. Hauptbestandteil ist ein feinfaseriger bis mikrokristalliner Aragonit in Form von unregelmäßigen, mm- bis $1 \mathrm{~cm}$ großen Klumpen (,thrombolite clots“). Darin befinden sich erekte, z.T. etwas radialbüschelig angeordnete Filamentspuren von Scytonema (Filamentdurchmesser 20-30 $\mu \mathrm{m}$ ). Teilweise ist noch ein fadenartiger, kollabierter Rest des ehemaligen Trichoms innerhalb einer röhrenförmigen Organikhülle (ehemalige Polysaccharidscheiden) zu erkennen. Ein Wechsel der Hohlraumgrößen führt zu einem grob stromatolithartigen Lagenbau im cm-Bereich. Die Hohlräume zeigen, im Gegensatz zum Lake Thetis, keine „Organomikrit“-Krusten. Das Internsediment der Hohlräume besteht aus Karbonatsand, gebildet von 100-1000 $\mu \mathrm{m}$ großen, angularen bis unregelmäßigen Intraklasten (wohl überwiegend Bioherm-Material) und einigen Ostrakoden-Schalen.

Interpretation: Unregelmäßige Hohlräume bilden über 50\% des Gesteinsvolumens. Sie bilden die ehemals schwach oder nicht mineralisierten Exopolymer-Bereiche der mikrobiellen Gemeinschaften ab und sind infolge subaerischer Exposition etwas lösungserweitert.

\subsection{Mikrobialithe, Lake Preston}

Beschreibung: Marine, holozäne Karbonatsande sind im Uferbereich des stark eingedunsteten Sees über weite Strecken von einer cm-dicken mikrobialithischen Kruste zementiert. Der marine Karbonatsand (Korngrößen 200-1000 $\mu \mathrm{m}$ ) besteht aus Mollusken- und Echinodermen-Bruchstücken, Foraminiferen und Rotalgenfragmenten (Taf. 11/A). Weiterhin sind Grobquarze $(200-800 \mu \mathrm{m})$ eingestreut. Im Bereich des trockenen Ufers wurden auch schlierig-laminierte und quarzsandreiche Caliche-Krusten in den Karbonatsanden beobachtet, welche makroskopisch z.T. stromatolithisch wirken (Taf. 11/B-C).

Die 2-3 cm dicke Mikrobialith-Kruste besteht aus feinfaserigem bis mikrokristallinem Aragonit mit unregelmäßigen, horizontal ausgelenkten Hohlräumen. Die Hohlräume sind stellenweise von einer laminierten „Organomikrit“-Kruste ausgekleidet (Taf. 11/D). Kompaktere Bereiche zeigen durch den Wechsel von grauen zu hellen Zonen ein grob stromatolithisches Gefüge. Der Aragonit zeigt erekte, teilweise radial-büschelförmig 
angeordnete Filamentspuren von Cyanobakterien (Taf. 11/F). Im Vergleich zu den Scytonema-Filamenten des Lake Clifton und Lake Pollard sind sie von geringerem Durchmesser (5-7 $\mu \mathrm{m}$, ,,Phormidium“ sp.).

\subsection{Mikrobialithe, Lake Walyungup}

\section{Fossile Thrombolithe}

Beschreibung: Krümelig-mikritischer bis mikrokristalliner Aragonit enthält Ansammlungen von dicht aneinanderliegenden, meist erekten Scytonema-Relikten (20 $\mu \mathrm{m}$ Durchmesser) und horizontal orientierten Cyanobakterien mit dickerer Gallertscheide (50-70 $\mu \mathrm{m}$ Durchmesser, vermutlich ebenfalls Scytonema-Morphotyp). Diese äußere, ehemals Polysaccharid-reiche Gallertscheide ist in Form von Hydromagnesit erhalten, das Trichom ist als mikritisches Zentrum erkennbar.

„Organomikrit“-Krusten, welche die Hohlräume ausfüllen, weisen eine rot-braune Färbung infolge von Verwitterung auf. Bis $\mathrm{zu} 1,8 \mathrm{~cm}$ breite und weiträumig oberflächenparallele „Organomikrit“-Krusten bestehen aus grauem bis rotbraunem Mikrit im Wechsel mit sehr feinen Zementeinschaltungen und belegen ein Stromatolith-Wachstum (Taf. 12/E). Die Stromatolithe trennen zum einen jüngere von älteren Thrombolith-Phasen (krümelige Aragonite). Sie bilden jedoch auch die abschließende Thrombolith-Oberfläche.

Interpretation: Erodierte Thrombolithe kommen zahlreich am Ufersaum des E-Ufers vor. Neben schollenartig-gebrochenen Karbonatkrusten zeichnen sich die bis zu 2 x 0,5 m großen Thrombolithe durch eine knollige Struktur aus (Taf. 12/A-B). Die permanent exponierten Thrombolithe werden aufgrund der Verwitterungskrusten als fossil eingestuft.

\section{Weiße Karbonatkrusten}

Beschreibung: Weiße Karbonatkrusten bilden sich am Wasserkontakt im Interlitoral direkt am Seeufer (Taf. 12/C-D). Unter mikroskopischer Betrachtung findet man darin unregelmäßige Mikritklumpen, an deren äußeren Bereichen sich pallisadenartige Zemente (Taf. 13/G-H) und dunkelbraune, mikrokristalline Säume absetzen können. Untergeordnet sind runde Strukturen als Cyanobakterien-Relikte erkennbar (Scytonema-Morphotyp). Gut erhaltene Scytonema-Filamente werden von einer grobkristallinen Zementphase (Hydromagnesit) in äußeren Schliffbereichen eingeschlossen und zeigen eine deutlich buschförmige Anordnung. 
Interpretation: Die Krusten werden als subrezente bis rezente Bildungen interpretiert. Inwiefern die Hydromagnesit-Zemente die primären Aragonite ersetzten (CoSHELL et al., 1998) oder selbst primär präzipitiert werden, wie von Hydromagnesit-Stromatolithen in dem alkalinen See Salda Gölü (Türkei) bekannt (BRAITHWAITE \& ZEDEF, 1996), kann mit Hilfe der angewendeten Methoden nicht geklärt werden. Aufgrund der gut erhaltenen Filamentspuren innerhalb der Zemente wird jedoch eine primäre Fällung favorisiert.

\subsection{Mikrobialithe, Shark Bay}

Beschreibung: Untersucht wurden die jüngsten $10 \mathrm{~cm}$ der subrezenten Stromatolithe sowie hart zementierte, zentimetergroße Mikrobialith-Polster auf den subfossilen Stromatolithen des Intertidals (Taf. 14/A-C).

Hauptbestandteil der klassischen agglutinierten Stromatolithe (Taf. 16/A-D) sind Lamellen aus lockerem, marinem Karbonatsand im Wechsel mit mikrokristallin zementierten Karbonatsandlagen (vgl. REID et al., 2003). Die lockeren Karbonatsandlagen (500 $\mu \mathrm{m}$ - 3 $\mathrm{mm})$ bestehen hauptsächlich aus Ooiden (120-140 $\mu \mathrm{m})$, Molluskenbruch (bis mm-Größe) und Foraminiferen (250-1000 $\mu \mathrm{m})$. Nur stellenweise ist ein äußerst dünner, nadeliger Aragonitzement entwickelt. Der Porenraum ist offen und die Komponenten sind in ihrer Internstruktur unverändert.

Entscheidend für die Lithifizierung und den Aufbau der Stromatolithe sind dagegen die mikrokristallin zementierten Karbonatsandlamellen $(300-500 \mu \mathrm{m})$. Hier sind die marinen Karbonatsandkörner zunehmend mikritisiert, selten sind lichtoptisch Endolithen zu erkennen (Taf. 16/G). Die Sandkörner werden von einem dichten, mikrokristallinen Karbonat zementiert (Taf. 16/C). Das stromatolithische Gefüge in den locker zementierten Karbonatsandlagen wird durch laminaeparallele Hohlräume betont (Taf. 16/A-B).

Subfossile Stromatolithe des Intertidals weisen an den Seiten zusätzlich knotige, grünliche Mikrobialith-Aufwüchse auf (Taf. 14/B-C). Sie bestehen aus mikrokristallinem Karbonat mit klumpigem Mikrogefüge und feinen, unregelmäßigen Poren. Makroskopisch und mikroskopisch ist keine stromatolithische Lamination erkennbar. Mikroskopisch zeigt sich jedoch durch einen Farbwechsel und lagig angeordnete Hohlräume ein schwach stromatolithisches Gefüge (Taf. 16/F). Das Karbonat ist von gelbbraunen Flecken (50-100 $\mu \mathrm{m}$ Durchmesser) durchsetzt, welche möglicherweise auf Polysaccharidscheiden kokkaler Cyanobakterien bzw. ihrer Mikrokolonien zurückgehen könnten. Agglutiniertes Karbonatmaterial fehlt, so dass eine mikrobielle Kalzifikation für den Aufbau dieser Strukturen entscheidend sein muss. 


\subsection{Vergleich von rezenten und fossilen Biofilmgemeinschaften}

\subsubsection{Lake Thetis}

Rezente Biofilme werden von Diatomeen dominiert, welche sich in den fossilen Mikrobialithen ausschließlich in Form von Diatomeen-Sanden in den Taschenfüllungen finden. Es wird vermutet, dass die Lösung der Diatomeen-Schalen aufgrund des organischen Schalenanteils erst nach Absterben und Zersetzung der Kieselalge einsetzt.

In den subfossilen Karbonaten konnten keine Gloeocapsa-Kolonien nachgewiesen werden. Eine Fehlerquelle hierfür kann sein, dass bei schlecht erhaltenem Teilungsmuster der Gloeocapsa sp. diese den unbestimmbaren, kokkalen Cyanobakterien oder dem Morphotyp Entophysalis sp. zugeordnet wurden.

Die rezenten Biofilme werden von Entophysalis- und Gloeocapsa-Kolonien sowie Scytonema sp. dominiert, wobei sich ein saisonaler Wechsel zeigt. Ein solcher ist in den fossilen Stromatolithen zwischen „Scytonema-Lamellen“ und „Entophysalis-Lamellen“ erkennbar, welcher allerdings aufgrund der cm-breiten Lamellen auf stabilere Umweltbedingungen schließen lässt.

Das Erscheinungsbild und die Häufigkeit von Scytonema sp. in den Frühjahrsproben deckten sich mit der lagenparallelen, erekten Orientierung der Filamente in den oberen StromatolithKrusten (Mikrobialith-Typen 3 und 4). In älteren Mikrobialithen sind Scytonema-Filamente nur untergeordnet und weniger in erekter Position erhalten. Der erhöhte Anteil an holozänem Schill und peloidalem Mikrit sind Anzeichen für einen erhöhten terrigenen Eintrag in einem Bewegtwasser-Milieu.

SPB sind in den fossilen Karbonaten ausschließlich aufgrund der Biomarker-Untersuchungen nachgewiesen. Eine Co-Existenz der SPB des Seebeckens mit den marginalen Stromatolithen ist somit nicht nur rezent, sondern auch für den „,fossilen“ Lake Thetis belegt.

\subsubsection{Lake Clifton}

Rezente Biofilmgemeinschaften im Lake Clifton werden von Diatomeen, Gloeocapsa sp. und filamentösen Cyanobakterien (u.a. Phormidium sp.) dominiert. Ältere sowie eigene Untersuchungen an den fossilen Thrombolithen belegen dagegen eine Verbreitung von Scytonema sp. und einer Vielzahl weiterer Cyanobakterien (MOORE \& BURNE, 1994). Es ist demnach ein Wandel der Cyanobakterien-Spezies erfolgt. Gründe hierfür können in Änderungen der eutrophen Bedingungen des Sees liegen. 


\section{Stabile Isotope}

Es wurden Proben fossiler und subfossiler Mikrobialithe, karbonatischer Sedimente und spherulithischer Aragonitpräzipitate untersucht. Alle Isotopendaten liegen im Kovarianz-Plot (Abb. 4.1) entlang eines breiten Korridors von typisch meteorischen Werten (Caliche des Lake Preston) bis hin zu deutlich schweren $\delta^{13} \mathrm{C}$ - und $\delta^{18} \mathrm{O}-$ Werten [PDB], wie sie in evaporitischen Systemen geschlossener Seen zu erwarten sind (ROTHE et al., 1974). Sie unterscheiden sich damit deutlich von marinen Werten und anschließenden Diagenesepfaden. Stärkere Abweichungen von der Regressionsgeraden aller Daten zeigen die Mikrobialithe des Lake Clifton und Lake Thetis sowie die Aragonitpräzipitate organikreicher Seebodensedimente des Lake Thetis (Abb. 4.1-4.6).

\subsection{Lake Thetis}

Datenbeschreibung: Cyanobakterielle Stromatolith-Lamellen zeigen $\delta^{13} \mathrm{C}=+0.73$ bis +3.46 $\% o$ und $\delta^{18} \mathrm{O}=+1.66$ bis $+3.04 \%$. Zur Verdeutlichung sind in Anhang 3 und 4 die Probenpunkte aus den einzelnen Lagen dargestellt. „Organomikrit“-Überzüge der Stromatolith-Bioherme weisen Werte von $\delta^{13} \mathrm{C}=+1.37$ bis $+2.80 \%$ und $\delta^{18} \mathrm{O}=+1.22$ bis $+2.41 \%$ auf. Die stabilen Isotopenwerte von Karbonatpartikeln des SPB-reichen Seebodensedimentes reichen bezüglich $\delta^{13} \mathrm{C}$ von -0.23 bis $-1.68 \%$ und bezüglich $\delta^{18} \mathrm{O}$ von +2.19 bis $+2.54 \%$ o. Die Spherulithe des Seebodensedimentes (Taf. 18/A-C) erreichen Werte von $\delta^{13} \mathrm{C}=-6.76$ bis $+3.12 \%$ ound von $\delta^{18} \mathrm{O}=+1.86$ bis $+3.01 \%$ Eine zum Vergleich entnommene Probe eines marinen holozänen Karbonatsandes lieferte $\delta^{13} \mathrm{C}=-0.70 \%$ und $\delta^{18} \mathrm{O}=+0.59 \%$ (Abb. 4.2, 4.3, 4.5 A + B; Anhang 5).

Interpretation: Beim Lake Thetis handelt es sich um ein geschlossenes, leicht hypersalinares Seebecken mit Oberflächenzufluss. Ein unterirdischer Kontakt zum Grundwasserkörper in holozänen Karbonatsanden ist nicht nachgewiesen, kann allerdings auch nicht ausgeschlossen werden.

Die Isotopen-Messungen zeigen eine relativ breite Streuung der $\delta^{13} \mathrm{C}$ - und $\delta^{18} \mathrm{O}$-Werte ohne deutliche Trends oder Kovarianzen, wie sie in einem durch Evaporation geprägten, geschlossenen Seebecken eigentlich zu erwarten gewesen wären (Abb. 4.2). Bereits GREY et al. (1990) bemerkten eine erhebliche Variation der stabilen Isotopen-Werte ohne erkennbaren Trend in der Zeit. Die im Vergleich zu angrenzenden holozänen und marinen Karbonatsanden hohen $\delta^{13} \mathrm{C}$-Werte interpretierten sie als Hinweis auf eine Karbonatfällung aus einer durch 

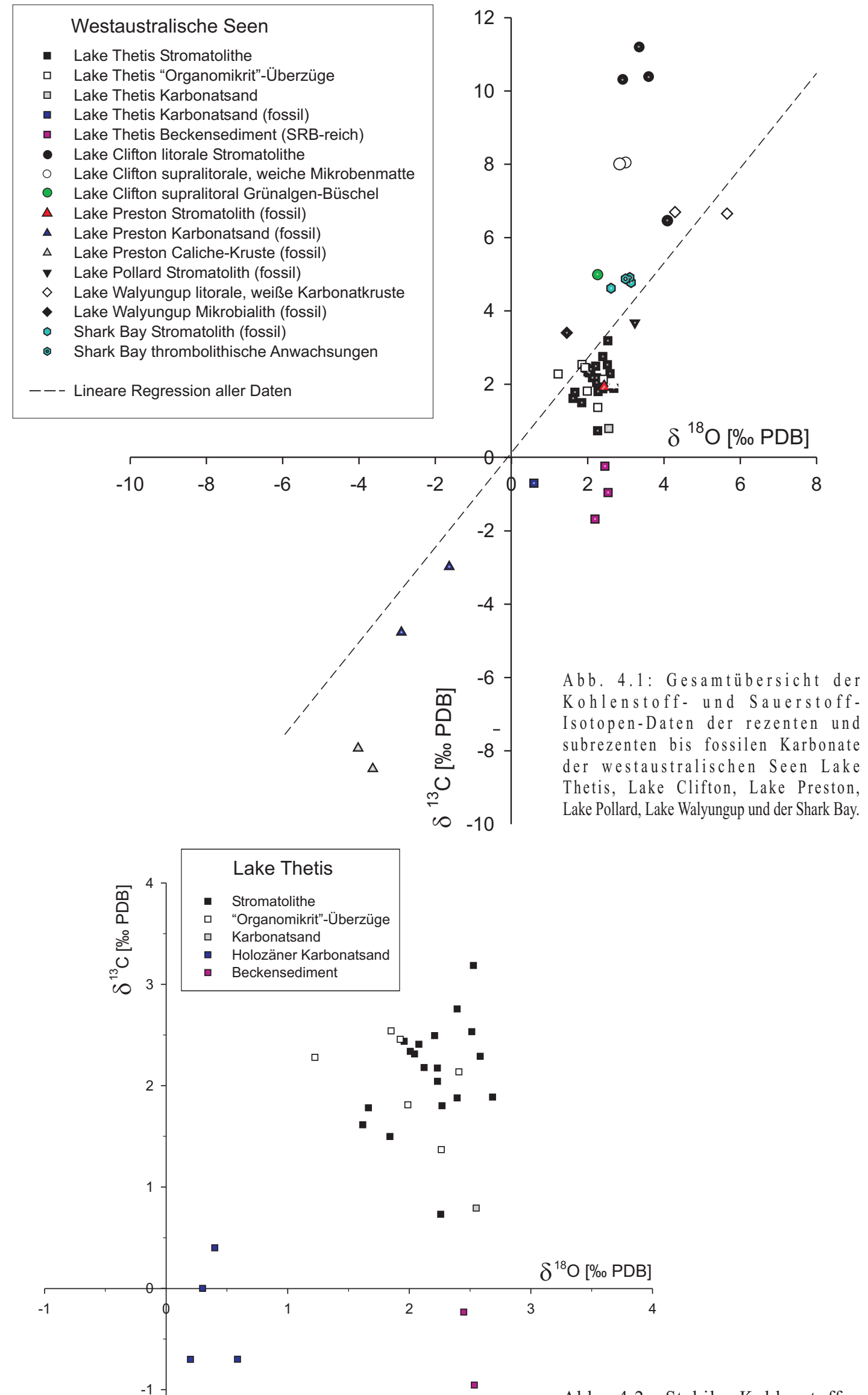

Abb. 4.2: Stabile Kohlenstoff- und Sauerstoff-Isotope der rezenten und subrezenten Karbonate des Lake Thetis. 
biologische Aktivität (Photosynthese) geprägten „Quelle“(d.h. DIC-Pool).

Im Gegensatz zum Kovarianz-Plot lassen die Stromatolith-Krusten im Profil allerdings eine negative Kovarianz erkennen (Abb. 4.3, 4.4). Im Vergleich $\mathrm{zu}$ den jeweils angrenzenden Cyanobakterien-Lamellen sind relativ schwere $\delta^{13} \mathrm{C}$-Werte mit leichteren $\delta^{18} \mathrm{O}$-Werten gekoppelt und umgekehrt. Es ergibt sich ein leichter Trend in der Zeit von gleichbleibenden bis schwerer werdenden hin zu leichteren $\delta^{18} \mathrm{O}-$ Werten (ab den Entophysalis- und ScytonemaLagen des mittleren Profilabschnittes der oberen Kruste). Die Isotopie des Kohlenstoffs weist z.T. starke Schwankungen mit einem übergeordneten Trend von schwerer werdenden Werten zu leichteren Werten auf, letztere sind in den oberen Lagen (Entophysalis, Scytonema und „Organomikrit“) ausgeprägt. Dabei sind oftmals - allerdings nur im tieferen Teil des Profils (Abb. 4.3) - „Entophysalis-Lamellen“ im Vergleich $\mathrm{zu}$ den jeweils vorhergehenden „Scytonema-Phormidium-Lamellen“ relativ schwerer bezüglich $\delta^{13} \mathrm{C}$ und leichter bezüglich $\delta^{18} \mathrm{O}$. Die ,Scytonema-Lamellen“ sind häufig relativ schwerer in $\delta^{13} \mathrm{C}$ und leichter in $\delta^{18} \mathrm{O}$. Diesem Muster ist phasenweise eine schwach positive Korrelation übergeordnet. Die Schwankungsbreite der Daten ist selbst in homogenen Lagen, wie der oberen „Organomikrit“Kruste, deutlich und zeigt sich verstärkt sowohl in den $\delta^{13} \mathrm{C}$-Werten, als auch in den $\delta^{18} \mathrm{O}$ Werten innerhalb der Scytonema-Lagen.

Eine Interpretation muss Effekte von Evaporation, Schwankungen der Primärproduktion, der Zufuhr meteorischer und sublakustriner Wässer und einer möglichen Wasserschichtung im See berücksichtigen. So erklären Woo et al. (2003) die negative Korrelation von $\delta^{13} \mathrm{C}$ und $\delta^{18} \mathrm{O}$ in lakustrinen Stromatolithen der Sinyangdong Formation (Mittel-Kreide, Korea) mit einer inversen Kopplung von Primärproduktion und Salinität.

Auf den Fall Lake Thetis kann dies allerdings nicht direkt übertragen werden. „EntophysalisLamellen“, welche während niedrigem Seespiegel (stärkere Evaporation) entstanden, zeigen oftmals niedrigere $\delta^{18} \mathrm{O}$-Werte (demnach niedrigere Evaporation) bei höheren $\delta^{13} \mathrm{C}$-Werten (höhere Primärproduktion im See). Bei „Scytonema-Phormidium-Lamellen“, die überwiegend während höherem Seespiegel entstanden, ist dies vielfach umgekehrt.

Es ist zu vermuten, dass im Flachstwasser eine Überschichtung mit meteorischem Wasser (niedrige $\delta^{18} \mathrm{O}$-Werte) $\mathrm{zu}$ einer Verdünnung, geringerer $\mathrm{pH}$-Pufferung und stärkerem Photosynthese-Effekt (höhere $\delta^{13} \mathrm{C}$-Werte) geführt hat. Schwere Werte lassen sich über eine Mischung mit evaporitisch geprägtem See- wie auch Grundwasser erklären. Die Streuungen der Sauerstoff-Isotopien im mittleren Teil (Lagen 4-6, Anhang 3 und 4) gehen vermutlich auf einen erhöhten Eintrag terrestrischen Materials (Taschenfüllungen) und geänderte Milieubedingungen zurück. 


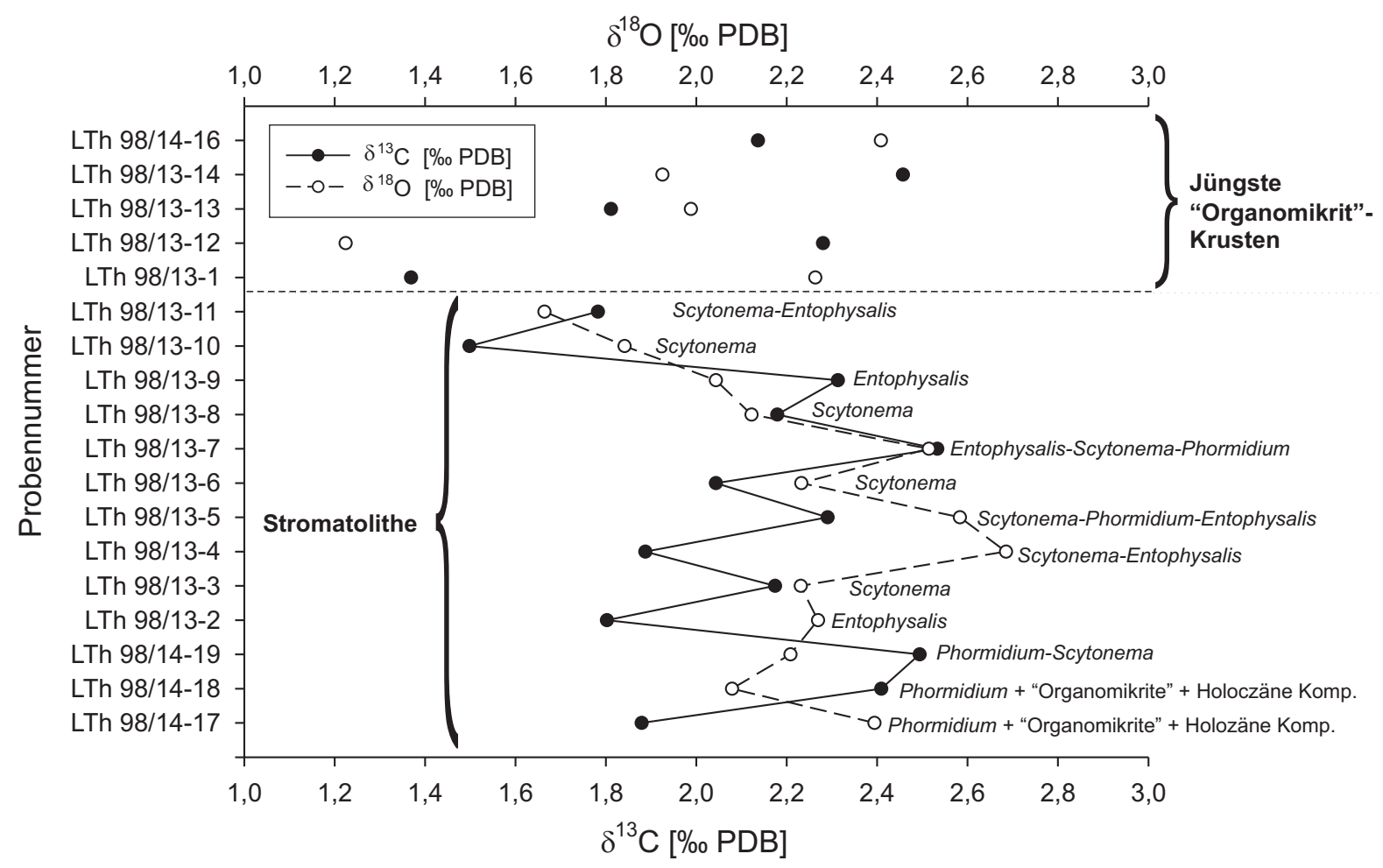

Abb. 4.3: Kohlenstoff- und Sauerstoff-Isotopen-Daten einzelner Wachstumsphasen der mittleren und äußeren Stromatolith-Krusten vom Lake Thetis.
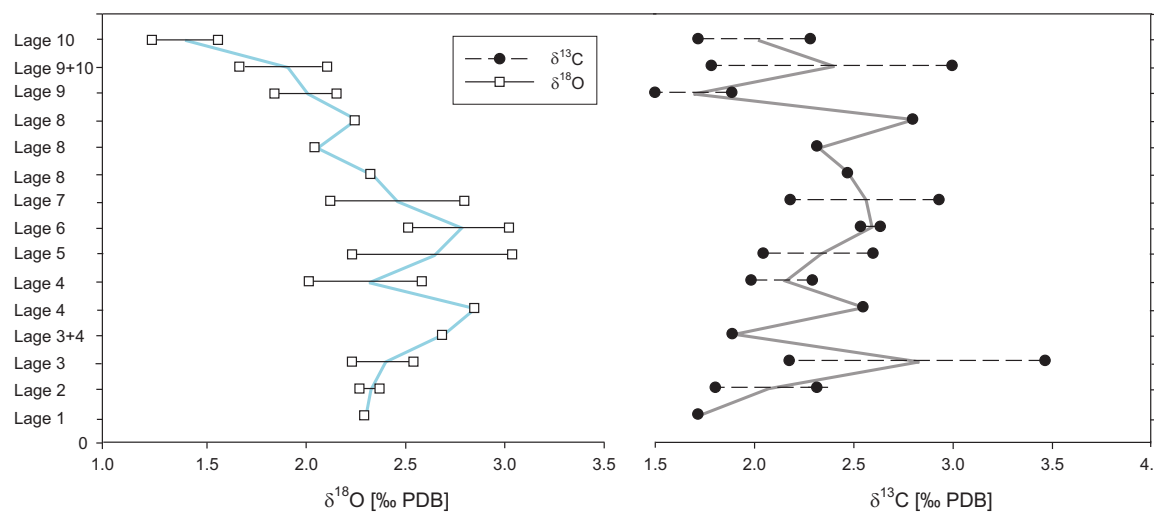

"Organomikrit"

Entophysalis-Lamelle (\& Scytonema) Scytonema-Lamelle Organomikrit Entophysalis \& "Organomikrit" Entophysalis-Lamelle Scytonema-Lamelle Scytonema-Lamelle Ecyonethame Scytonema-Lamelle "Organomikrit" (\& Entophysalis) Entophysalis-Lamelle Entophysalis-Lamelle (\& Scytonema) Scytonema-Lamelle Entophysalis-Lamelle Phormidium

Abb. 4.4: Darstellung der Kohlenstoff- und Sauerstoff-Isotopen-Daten (Proben LTh 98/13- 2 bis 12 und LTh 98/13-51 bis 63, vgl. Abb. 4.3 und Anhang 3, 4) der Wachstumsphasen der äußeren Stromatolith-Kruste vom Lake Thetis. Die Datenpunkte sind Einzelmesswerte, die horizontalenVerbindungslinien verbinden zwei Datenpunkte, deren Proben aus denselben Lagen stammen. Die vertikalen Verbindungslinien verdeutlichen den Trend in der Zeit zu leichter werdenden Werten im oberen Teil. 
Ein zusätzlicher Eintrag leichteren Boden- $\mathrm{CO}_{2}$ ist denkbar und würde speziell innerhalb der bei Seespiegelhochstand entstandenen „Scytonema-Lamellen“ zu einer Streuung führen. Zudem führt sicherlich die Probennahme innerhalb des inhomogenen Materials (insbesondere Mg-Si-reiche „Scytonema-Lamellen“) und den schlierig verlaufenden Lagengrenzen zur Produktion von Mischwerten.

Ein weiteres Kriterium sind innerhalb des Biofilms rasch wechselnde Mikrogradienten, welche durch Einflüsse der pH-Pufferung, Photosynthese, Wasserüberschichtung, der Primärproduktion organischen Materials und durch Diffusionslimitierung (vgl. JAHNKE et al., 2004) die Werte beeinflussen können. Bei herabgesetzter Diffusion findet kaum ein Isotopenausgleich mit dem Seewasser statt. Eine Fraktionierung ist dann von dem im Mikromilieu enthaltenen Kohlenstoff abhängig, dessen Kohlenstoff-Isotopie wiederum mit der Primärproduktion im Zusammenhang steht. Leider fehlen für einen Vergleich IsotopenDaten des Seewassers.

Ein weiterer auffallender Aspekt im Kovarianz-Plot der stabilen Isotopen-Werte vom Lake Thetis ist die im Vergleich zu den Mikrobialithen deutlich ins Negative verschobene Kohlenstoff-Isotopie der aragonitischen Fällungsprodukte (Spherulithe) des organikreichen Seebodensedimentes (Abb. 4.5A ). Allerdings bleiben die $\delta^{18} \mathrm{O}$-Werte von Mikrobialithen und Spherulithen im gleichen Bereich.

Dies kann mit einem photosynthestischen ${ }^{12} \mathrm{C}$-Entzug in den oberen Wasserschichten und dem Transfer primär produzierter Organik ins Seebodensediment interpretiert werden. Dadurch erscheinen Mikrobialithe des Flachwassers relativ ${ }^{13} \mathrm{C}$-angereichert. Hinzu kommt vermutlich

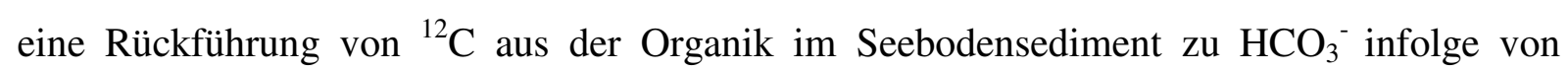
Sulfatreduktion, wodurch Spherulithe zusätzlich an ${ }^{12} \mathrm{C}$ angereichert werden.

\subsection{Lake Clifton}

Datenbeschreibung: Biohermkarbonate (aragonitische Thrombolithe des Eulitoral) zeigen $\delta^{13} \mathrm{C}=+6.47$ bis $+11.21 \%$ und $\delta^{18} \mathrm{O}=+2.91$ bis $+4.09 \%$ o. Karbonatpartikel weicher Mikrobenmatten (Supralitoral) weisen Werte von $\delta^{13} \mathrm{C}=+8.00$ bis $+8.07 \%$ o und $\delta^{18} \mathrm{O}=+2.86$ bis $+3.02 \%$ auf. Eine Probe von Karbonatüberzügen der Grünalgenbüscheln (Supralitoral) lieferte $\delta^{13} \mathrm{C}=+5.00 \%$ und $\delta^{18} \mathrm{O}=+2.25 \%$ (Abb. 4.6, Anhang 5).

Interpretation: Der Lake Clifton ist ein durch Grundwasser-Zutritte geprägtes evaporitisches System (MOORE et al., 1983, MoORE, 1987; 1993). Die gemessenen Biohermkarbonate sind auffallend schwer bezüglich $\delta^{13} \mathrm{C}$, während $\delta^{18} \mathrm{O}$-Werte in einem kleinen Feld zwischen +2 und $+4 \%$ o liegen (Abb. 4.6). 

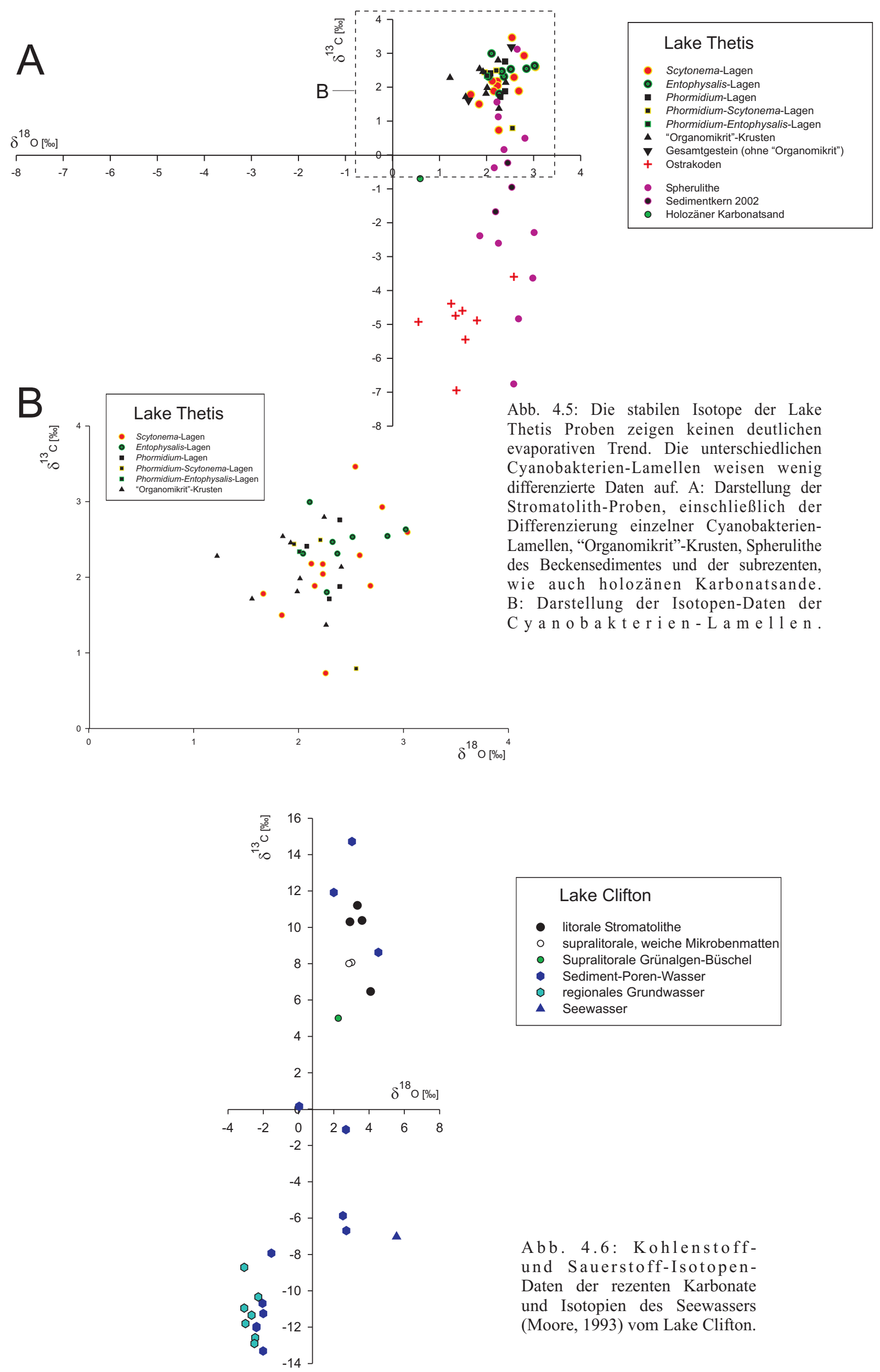

Abb. 4.6: Kohlenstoffund Sauerstoff-IsotopenDaten der rezenten Karbonate und Isotopien des Seewassers (Moore, 1993) vom Lake Clifton. 
Ähnlich schwere $\delta^{13} \mathrm{C}$-Werte wurden bereits von MOORE (1993) angegeben. Die Werte der weichen Mikrobenmatten des Supralitorals spiegeln eine Mischung aus Biohermdetritus, Bioklasten und allochthonen Karbonatpartikeln wider. Sie sind im Vergleich zu den Biohermen in Richtung meteorisches Grundwasser verschoben, wie es ebenfalls für die Karbonatüberzüge auf Grünalgenbüscheln des Supralitoral anzunehmen ist. Sie liegen im deutlichen Einflussbereich der oberflächennahen Süßwasserzufuhr am E-Ufer des Sees. Ihre Isotopie ist in Richtung meteorischer Werte verschoben, bleibt aber durch den gleichzeitigen Evaporationseffekt weit im positiven Bereich. Letzteres ist vermutlich durch die Zufuhr salinaren Wassers aus dem Ästuar verstärkt (vgl. Kap. 7.1).

Die enorm schweren $\delta^{13} \mathrm{C}$-Werte der Bioherme sind nur mit der Kenntnis der Isotopie von Seewasser, Sedimentporenwasser und Grundwasser zu interpretieren. Hierzu wird auf Daten von MOORE (1993) zurückgegriffen (Abb. 4.6). Allerdings ist das hydrologische System des Lake Clifton in den letzten Jahren starken, vorwiegend anthropogen verursachten Veränderungen (Erhöhung der Salinität und der Nährstoffzufuhr) ausgesetzt, so dass Zurückhaltung bei einer Verknüpfung aktueller Daten mit Daten aus der Literatur geboten ist. Das Seewasser wird von MoORE (1993) mit $\delta^{13} \mathrm{C}=-7.02 \%$ und $\delta^{18} \mathrm{O}=+5.56 \%$ angegeben. Meteorische Grundwässer der Umgebung liegen bei $\delta^{13} \mathrm{C}=-8.71$ bis $-12.58 \%$ und $\delta^{18} \mathrm{O}=-2.28$ bis $-3.08 \%$ o. Isotopen-Daten eines Querprofils durch den See (,transect T3“) wurden von MOORE (1993) mittels 2 m tiefer Sedimentkerne erstellt. Während am E-Ufer (mit Thrombolith-Biohermen) leichte, nahezu meteorische Werte im Porenwasser gemessen wurden, tritt am Westufer ein begrenzter Bereich mit schweren $\delta^{13} \mathrm{C}$-Werten in der Größenordnung der Biohermkarbonate auf $\left(\delta^{13} \mathrm{C}=+8.62\right.$ bis $+14.72 \%$ ). Dieser begrenzte Bereich ist an die Zufuhr salinarer Grundwässer (10-13 g/L) aus dem Westen gebunden, wobei die Wässer mit der höchsten Salinität (27-88 g/L) jedoch niedrigere $\delta^{13} \mathrm{C}$-Werte aufweisen, eventuell als Folge angrenzender Bodenbildung. Die Ursache dieser positiven $\delta^{13} \mathrm{C}$-Anomalie im Grundwasser ist unbekannt, könnte jedoch in den höher salinaren und

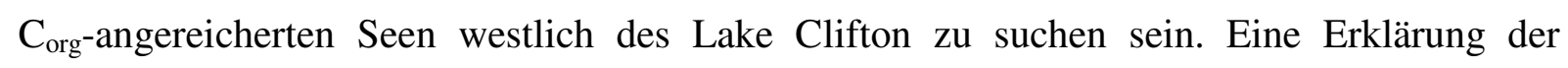
schweren Isotopenwerte der Bioherme durch eine photosynthesebedingte Fraktionierung von $\delta^{13} \mathrm{C}=-7 \%$ (Seewasser) auf $+10 \%$ (Biohermkarbonate), wie sie MooRE (1993) vorschlägt, erscheint unwahrscheinlich. Eine endgültige Klärung des Problems ist leider zur Zeit aufgrund der geringen Zahl eigener Messwerte und vor allem aufgrund fehlender IsotopenUntersuchungen der Seewasserproben nicht möglich. 


\subsection{Lake Walyungup}

Datenbeschreibung: Die fossile Mikrobialith-Probe mit $\delta^{13} \mathrm{C}=+3.40 \%$ und $\delta^{18} \mathrm{O}=+1.45 \%$ ist deutlich an ${ }^{13} \mathrm{C}$ und ${ }^{18} \mathrm{O}$ im Vergleich zu den ,frischen“ weißen KarbonatKrusten des Seeufers $\left(\delta^{13} \mathrm{C}=+6.66\right.$ und $+6.70 \%$; $\delta^{18} \mathrm{O}=+4.29$ und $+5.65 \%$ o $)$ abgereichert (Abb. 4.1).

Interpretation: Die Unterschiede zwischen fossilen und rezenten Mikrobialithen werden als Resultat einer zunehmenden Eindunstung des Sees interpretiert. Der vormals größere See ist seit mehreren Jahren in 3 Teilseen untergliedert (vgl. CosHELL et al., 1998), von denen der nördlichste trocken gefallen ist, und nur der mittlere und südliche Teil wasserführend sind. Als Grund hierfür wird eine Änderung des Grundwasserreservoirs angenommen. 


\section{Mineral- und Elementuntersuchungen}

\subsection{Röntgenuntersuchungen}

Zur mineralogischen Charakterisierung der karbonatischen Mikrobialithe wurden Röntgenbeugungsuntersuchungen durchgeführt. Die mineralogische Zusammensetzung der Mikrobialithe des Lake Thetis und des Lake Clifton ist im Wesentlichen aragonitisch (Tab. 5.1, Anhang 6a, 6b). Die Probe LTh 98/9 enthält zu dem noch Kalzit aus den holozänen Biound Extraklasten sowie Halit als Hintergrund des salinaren Seewassers. Die Mikrobialithe vom Lake Preston und vom Lake Pollard bestehen ebenfalls aus Aragonit mit untergeordneten Anteilen an Kalzit und Quarz. Fossile Stromatolithe der Shark Bay bestehen aus Aragonit und Quarz.

Die Proben vom Lake Walyungup weisen eine von den übrigen Mikrobialithen abweichende Mineralogie auf und bestehen aus Aragonit, Hydromagnesit und geringen Anteilen an Kalzit.

\begin{tabular}{|c|c|c|c|c|c|c|c|}
\hline \multirow[b]{2}{*}{$\begin{array}{l}\text { Lokalität, } \\
\text { Probennummer }\end{array}$} & \multirow{2}{*}{$\begin{array}{c}\text { Hauptgemengteile } \\
\text { Aragonit }\end{array}$} & \multicolumn{6}{|c|}{ Nebengemengteile \& Spuren } \\
\hline & & Kalzit & Halit & Quarz & $\begin{array}{c}\text { Ton- } \\
\text { minerale }\end{array}$ & Dolomit & $\begin{array}{c}\text { Hydro- } \\
\text { magnesit }\end{array}$ \\
\hline $\begin{array}{l}\text { Lake Thetis, } \\
\text { LTh } 98 / 13\end{array}$ & $\mathrm{X}$ & & & & & & \\
\hline $\begin{array}{l}\text { Lake Thetis, } \\
\text { LTh 98/14 }\end{array}$ & $\mathrm{X}$ & & & & & & \\
\hline $\begin{array}{l}\text { Lake Thetis, } \\
\text { LTh } 02 / 9\end{array}$ & $\mathrm{X}$ & $\mathrm{X}$ & $\mathrm{X}$ & & & & \\
\hline $\begin{array}{l}\text { Lake Clifton, } \\
\text { LCl 02/5 }\end{array}$ & $\mathrm{X}$ & & $\mathrm{X}$ & & $\mathrm{X}$ & & \\
\hline $\begin{array}{l}\text { Lake Clifton, } \\
\text { LCl 02/3 }\end{array}$ & $\mathrm{X}$ & & $\mathrm{X}$ & & $\mathrm{X}$ & & \\
\hline $\begin{array}{l}\text { Lake Preston, } \\
\text { LPr 02/3 }\end{array}$ & $X$ & & $\mathrm{X}$ & $\mathrm{X}$ & & & $X$ \\
\hline $\begin{array}{l}\text { Lake Pollard, } \\
\text { LPo 98/4 }\end{array}$ & $\mathrm{X}$ & $\mathrm{X}(1 \%)$ & & & & $\mathrm{X}(3 \%)$ & \\
\hline $\begin{array}{l}\text { Lake Walyungup, } \\
\text { LWa } 02 / 3\end{array}$ & $\mathrm{X}$ & & & & & & $\mathrm{X}$ \\
\hline $\begin{array}{l}\text { Lake Walyungup, } \\
\text { LWa 02/6 }\end{array}$ & $\mathrm{X}$ & $\mathrm{X}$ & & & & & \\
\hline $\begin{array}{l}\text { Shark Bay, } \\
\text { SB 98/29 }\end{array}$ & $\mathrm{X}$ & & $\mathrm{X}$ & $\mathrm{X}$ & & & \\
\hline
\end{tabular}

Tab. 5.1: Ergebnisse der röntgenographischen Untersuchungen (Anhang 6a-d). 


\subsection{Elementverteilungen}

Die Chemie der Karbonate wurde nach Säureaufschluss mittels ICP-OES ermittelt. Für eine nahezu rein aragonitische Zusammensetzung enthalten die Proben zu wenig Ca. Die Gehalte liegen beim Lake Thetis und Lake Clifton bei max. 35 Gew.-\% Ca (349 g/kg, Tab. 5.2), wodurch sich ein Gesamtkalziumkarbonat-Gehalt von nur 80-85 Gew.-\% ergibt (vgl. Kap. 6.1). Insbesondere der Thrombolith vom Lake Clifton enthält mit 6,5 Gew.-\% Mg für einen reinen Aragonit $\mathrm{zu}$ viel $\mathrm{Mg}$. Tonminerale als mögliche Träger des $\mathrm{Mg}$ wurden röntgenographisch ausschließlich beim Lake Clifton und hier auch nur untergeordnet nachgewiesen.

Die höchsten Mg-Gehalte (104 g/kg, Anhang 8) ergeben sich für die Proben vom Lake Walyungup durch den Hydromagnesit-Anteil.

\begin{tabular}{|c|c|c|c|c|c|c|}
\hline Probe & & $\begin{array}{c}\mathrm{Ca} \\
{[\mathrm{g} \mathrm{kg}-1]}\end{array}$ & $\begin{array}{c}\mathrm{Mg} \\
{[\mathrm{g} \mathrm{kg}-1]}\end{array}$ & $\begin{array}{c}\mathrm{Sr} \\
{[\mathrm{g} \mathrm{kg}-1]}\end{array}$ & $\begin{array}{c}\mathrm{Ba} \\
{[\mathrm{g} \mathrm{kg}-1]}\end{array}$ & $\begin{array}{c}\mathrm{Ca} / \mathrm{Sr}- \\
\text { Verhältnis }\end{array}$ \\
\hline LTh $98 / 13$ & $\begin{array}{l}\text { oberste Lage } \\
\text { "Organomikrit" }\end{array}$ & 329,30 & 14,74 & 5,69 & 0,05 & 57,88 \\
\hline LTh $98 / 13$ & Entophysalis-Lamelle & 334,80 & 12,57 & 5,45 & 0,05 & 61,44 \\
\hline & $\begin{array}{l}\text { Scytonema- } \\
\text { Entophysalis- }\end{array}$ & & & & & \\
\hline LTh $98 / 13$ & "Organomikrit" & 339,23 & 11,25 & 5,52 & 0,04 & 61,40 \\
\hline LTh $98 / 13$ & interner „Organomikrit“ & 339,08 & 11,13 & 5,27 & 0,04 & 64,31 \\
\hline LTh $98 / 14$ & $\begin{array}{l}\text { Gesamtgestein } \\
\text { (,Organomikrit"-reich) }\end{array}$ & 337,43 & 13,34 & 5,34 & 0,04 & 63,19 \\
\hline LTh 98/14 & Gesamtgestein & 349,65 & 10,58 & 5,36 & 0,04 & 65,28 \\
\hline LTh $98 / 14$ & $\begin{array}{l}\text { Gesamtgestein (oberer } \\
\text { Teil) }\end{array}$ & 338,03 & 15,54 & 5,34 & 0,05 & 63,35 \\
\hline LTh $98 / 12$ & Taschenfüllungen & 336,63 & 16,79 & 4,99 & 0,04 & 67,52 \\
\hline LTh 98/12 & „Organomikrit"-reich & 312,37 & 33,49 & 3,34 & 0,03 & 93,56 \\
\hline LCI 02/5 & Gesamtgestein & 154,21 & 65,54 & 1,00 & 0,09 & 154,70 \\
\hline LWa 02/5 & weiße Kruste & 204,88 & 99,42 & 3,11 & 0,05 & 65,90 \\
\hline LWa 02/3 & fossiler Mikrobialith & 185,23 & 104,78 & 2,95 & 0,05 & 62,83 \\
\hline SB 98/29 & $\begin{array}{l}\text { Thrombolithische } \\
\text { Anwachsungen }\end{array}$ & 351,00 & 1,55 & 9,52 & 0,01 & 36,88 \\
\hline
\end{tabular}

Tab. 5.2: Haupt- und Spurenelemente innerhalb einiger subfossiler Mikrobialith-Typen vom Lake Thetis, Lake Clifton, Lake Walyungup und der Shark Bay (Anhang 8). 


\subsection{ElektronenstrahImikrosonden-Analysen}

Obwohl die Röntgenbeugungsuntersuchungen meist Aragonit als Hauptmineral belegen und weitere Mineralphasen nur als Spuren nachweisbar waren, betrugen die aus der chemischen Analyse ermittelten Gesamtkarbonat-Gehalte lediglich 80-85 Gew.-\%. Zur höheren räumlichen Auflösung und zum eventuellen Nachweis röntgenamorpher Phasen wurden Mikrosonden-Analysen an zwei Proben der subfossilen Karbonate des Lake Thetis (LTh 98/13, Stromatolith-Typ 4 und LTh 98/14, Stromatolith-Typ 3) sowie an einer Probe der kegeligen Bioherme des Lake Clifton ( $\mathrm{LCl}$ 02/7) durchgeführt.

Die Ergebnisse sind als Oxide gegen den Messpunkt aufgetragen. Mit Hilfe der optischen Darstellung der Einschusspunkte, die bei der Messung entstehen, kann jeder einzelne Messpunkt den jeweiligen Analyse-Daten zugeordnet werden. So ergibt sich ein deutliches Verteilungsmuster der Elemente in Bezug auf einzelne Mineralphasen. Zusätzlich lässt sich eine offenbar röntgenamorphe $\mathrm{Mg}$-Si-Phase insbesondere in den untersuchten Mikrobialithen nachweisen.

\subsubsection{Subfossile Mikrobialithe, Lake Thetis}

Der Karbonatanteil der „Organomikrit“-Krusten und mikrokristallinen Aragonite beträgt zwischen 96 und 99 Gew.-\% $\mathrm{CaCO}_{3}$ und zeigt innerhalb einzelner Phasen nur geringe Schwankungen (Tab. 5.3, Abb. 5.1, 5.6-5.8). Innerhalb der Aragonit-Phase (,Scytonema- und Entophysalis-Lamellen“) zeigen sich durch die eingeschaltete $\mathrm{Mg}$-Si-Phase Schwankungen in den $\mathrm{CaO}-G e h a l t e n(A b b .5 .2)$.

Die Sr-Gehalte der „Organomikrite“ (Zemente und mikrokristalliner Aragonit) und klumpigen Aragonite weichen leicht voneinander ab. Tendenziell zeigen sich höhere Gehalte in den mikritischen „Organomikrit“-Lagen. Eine Ausnahme stellen die Gehalte von Line 5 dar. Die Sr-Gehalte sind ca. um 1000 ppm geringer als die der anderen Proben. Die höchsten SrGehalte liefern hier die Zementzwischenlagen mit 5800 ppm. Eine $375 \mu \mathrm{m}$ dicke Zementlage mit gleichbleibender Elementzusammensetzung spiegelt sehr konstante Bildungsbedingungen wider, z.B. stabile aquatische Bedingungen und Ionenzufuhr. Schwankungen im SrO-Gehalt einer weiteren Zementlage (Abb. 5.7) entstehen durch Mischwerte mit mikrokristallinen Einschaltungen oder Einschlüssen. Die „Organomikrite“ zeigen sowohl in den Zementphasen als auch in den dichten Lagen nahezu kein $\mathrm{MgO}$ und $\mathrm{SiO}_{2}$. Dass teilweise dennoch Zwischenräume innerhalb der „Organomikrite“ die Mg-Si-Phase enthalten, zeigt Abb. 5.2 (Messpunkte 92 und 95). 
Die positiven Korrelationen zwischen $\mathrm{CaO}-\mathrm{SrO}$ einerseits und $\mathrm{MgO}-\mathrm{SiO}_{2}$ andererseits sowie die negative Korrelation zwischen $\mathrm{CaO}-\mathrm{SrO}$ zu MgO-SiO${ }_{2}$ werden als Beleg für eine nahezu Mg-freie Karbonatphase gewertet (Abb. 5.5). Ein leichter Untergrund des Mg-Signals von max. 0,7 Gew.-\% $\mathrm{MgO}$ ist auch in Mg-Si-reinen Phasen zu beobachten. Dieser stammt vermutlich aus den Karbonaten. Tonminerale als Träger des $\mathrm{Mg}^{2+}$ sind über röntgenographische Untersuchungen nicht nachgewiesen.

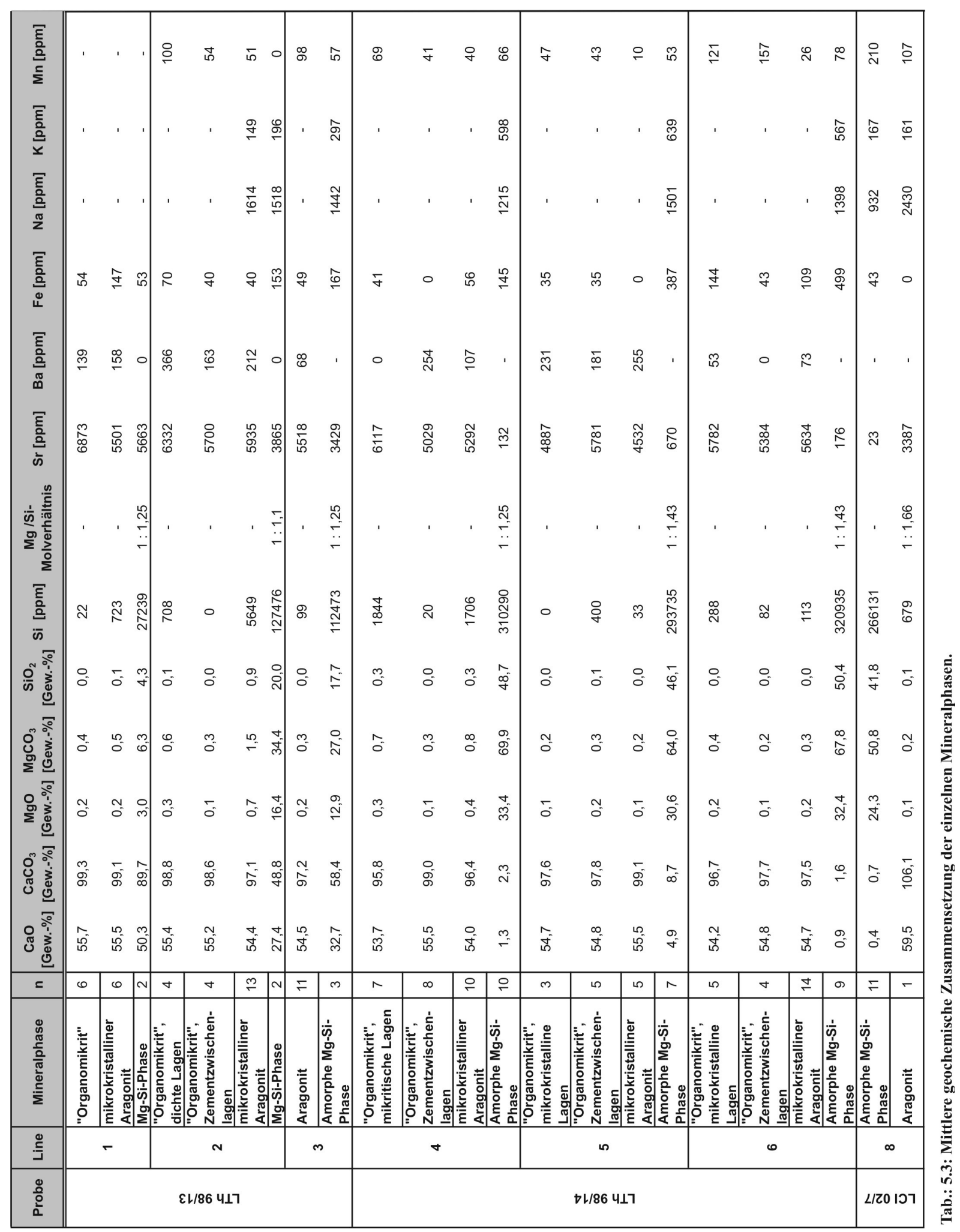




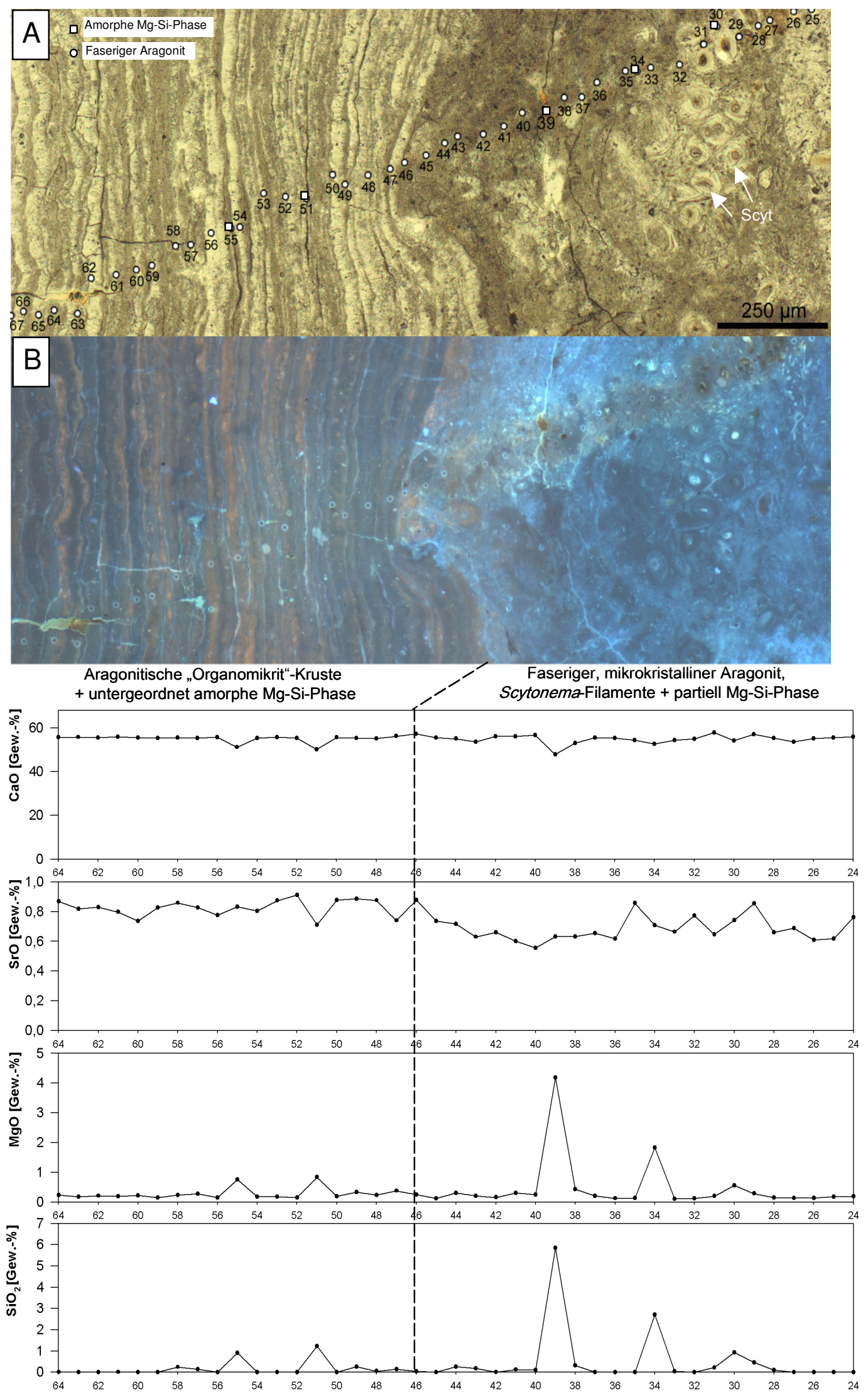

Abb. 5.1: Haupt- und Spurenelementverteilung. Probe Lake Thetis LTh 98/13, Line 1. A:

Hellfeld-Mikroskopaufnahme. B: UV-Filter-Mikroskopaufnahme. Scyt: Scytonema-Filamente. 


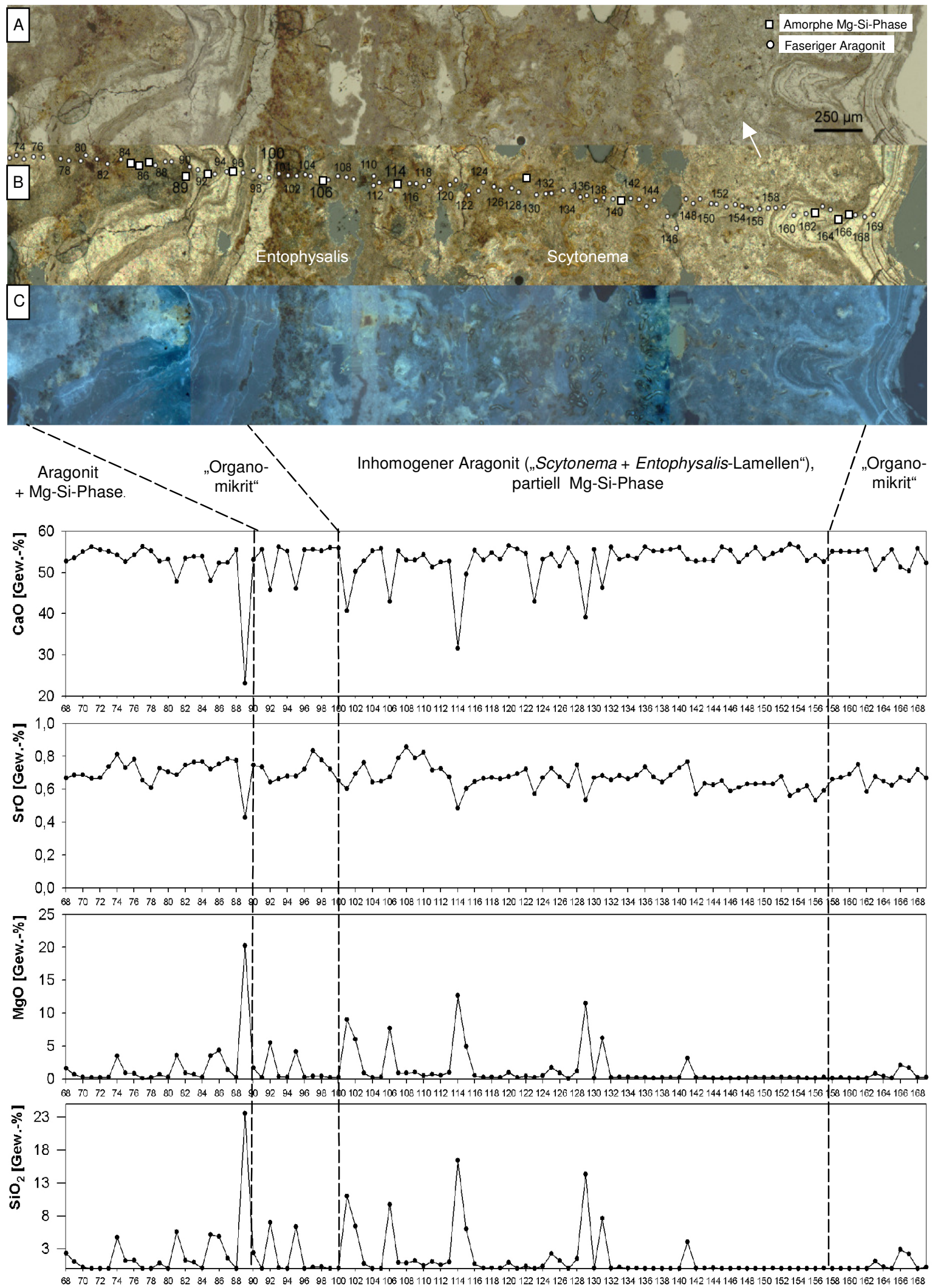

Abb. 5.2: Hauptelementverteilung. Probe Lake Thetis LTh 98/13, Line 2. A: HellfeldMikroskopaufnahme. B: Mikroskopaufnahme mit gekreuzten Polarisatoren. C: UV-FilterMikroskopaufnahme. Scyt: Scytonema-Filamente. 


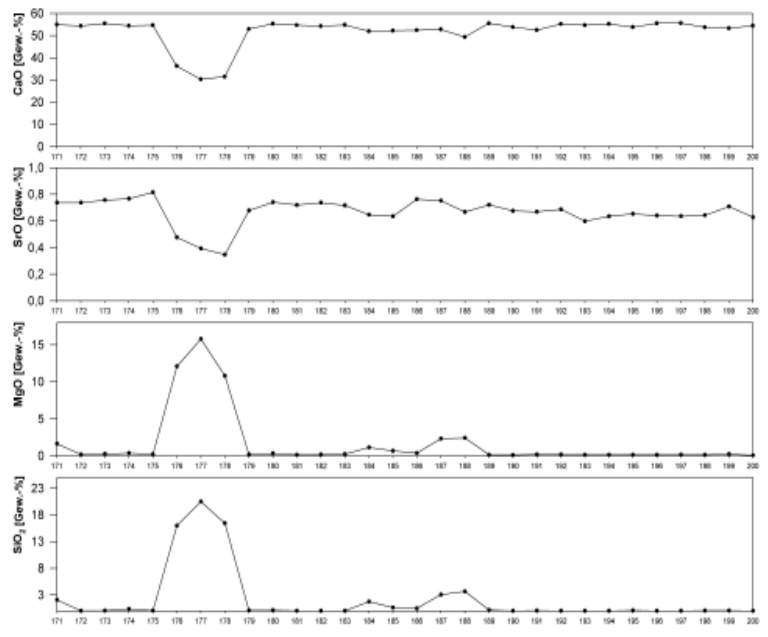

Abb. 5.3: Haupt- und Spurenelementverteilung. Probe Lake Thetis LTh 98/13, Line 3.
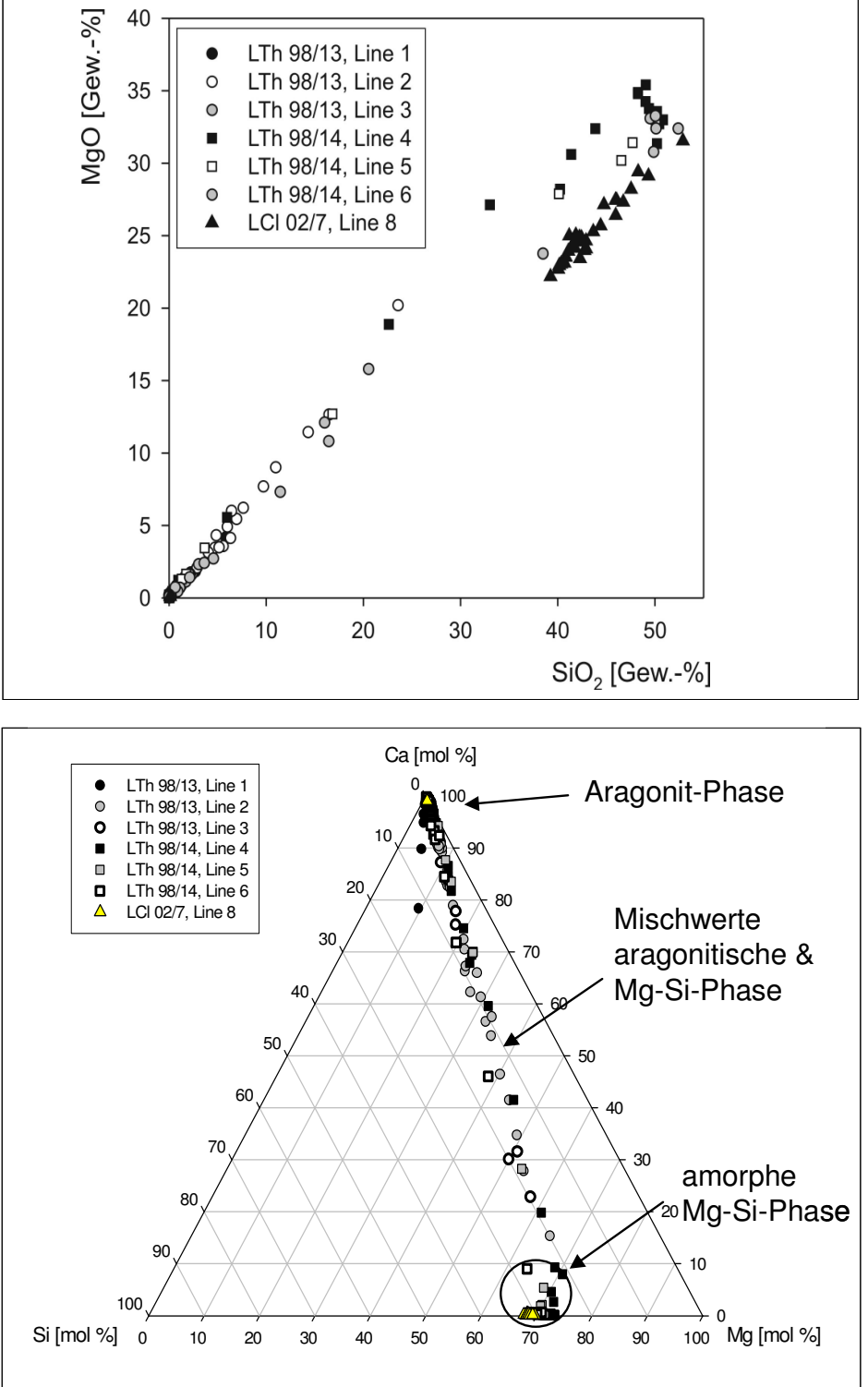

Abb. 5.4: Die Proben von Lake Thetis und Lake Clifton liefern Verteilungsmuster, welche die unterschiedlichen $\mathrm{Mg} / \mathrm{Si}$-Molverhältnisse von 1:1 und 1:1,25 (Lake Thetis) sowie 1:1,66 (Lake Clifton) repräsentieren.

Abb. 5.5: $\quad \mathrm{Ca}-\mathrm{Mg}-\mathrm{Si}-$ Verhältnisse aller untersuchten Probenpunkte. Die Abhängigkeit zwischen $\mathrm{Mg}$ und Si wird deutlich. Ca-reiche Proben enthalten nahezu kein $\mathrm{Mg}$ und Si. Mischwerte der Aragonit- und der $\mathrm{Mg}$-SiPhase bilden eine Linie zwischen den Endphasen. Eine Mg-Kalzit-Phase würde auf der Mg-Ca-Flanke des Diagramms plotten. 


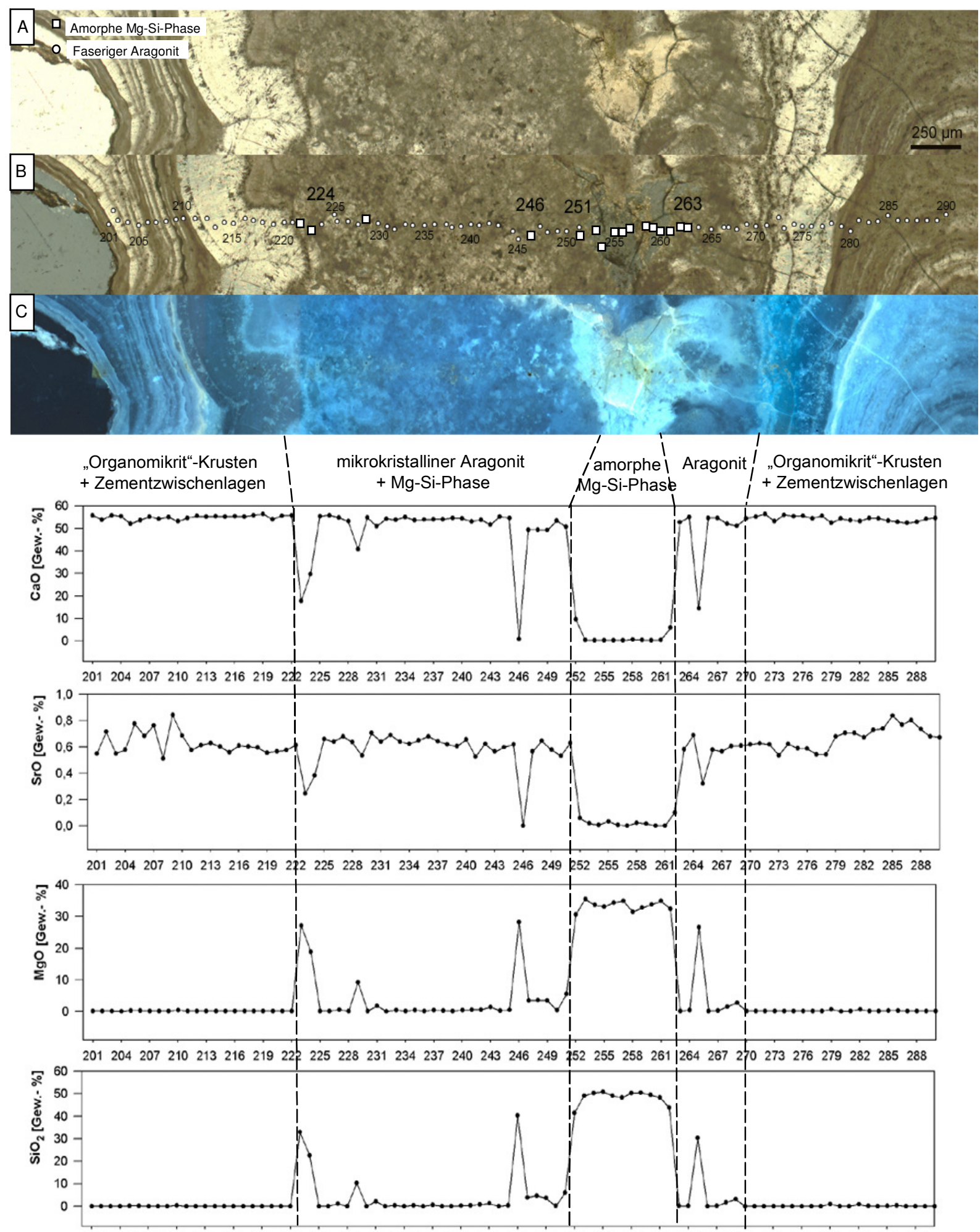

$201204207210213216219222225228231234237240243246249252255258261264267270273276279282 \quad 285288$

Abb. 5.6: Haupt- und Spurenelementverteilung. Probe Lake Thetis LTh 98/14, Line 4. A: Hellfeld-Mikroskopaufnahme. B: Mikroskopaufnahme mit gekreuzten Polarisatoren. C: unter UV-Anregung. 


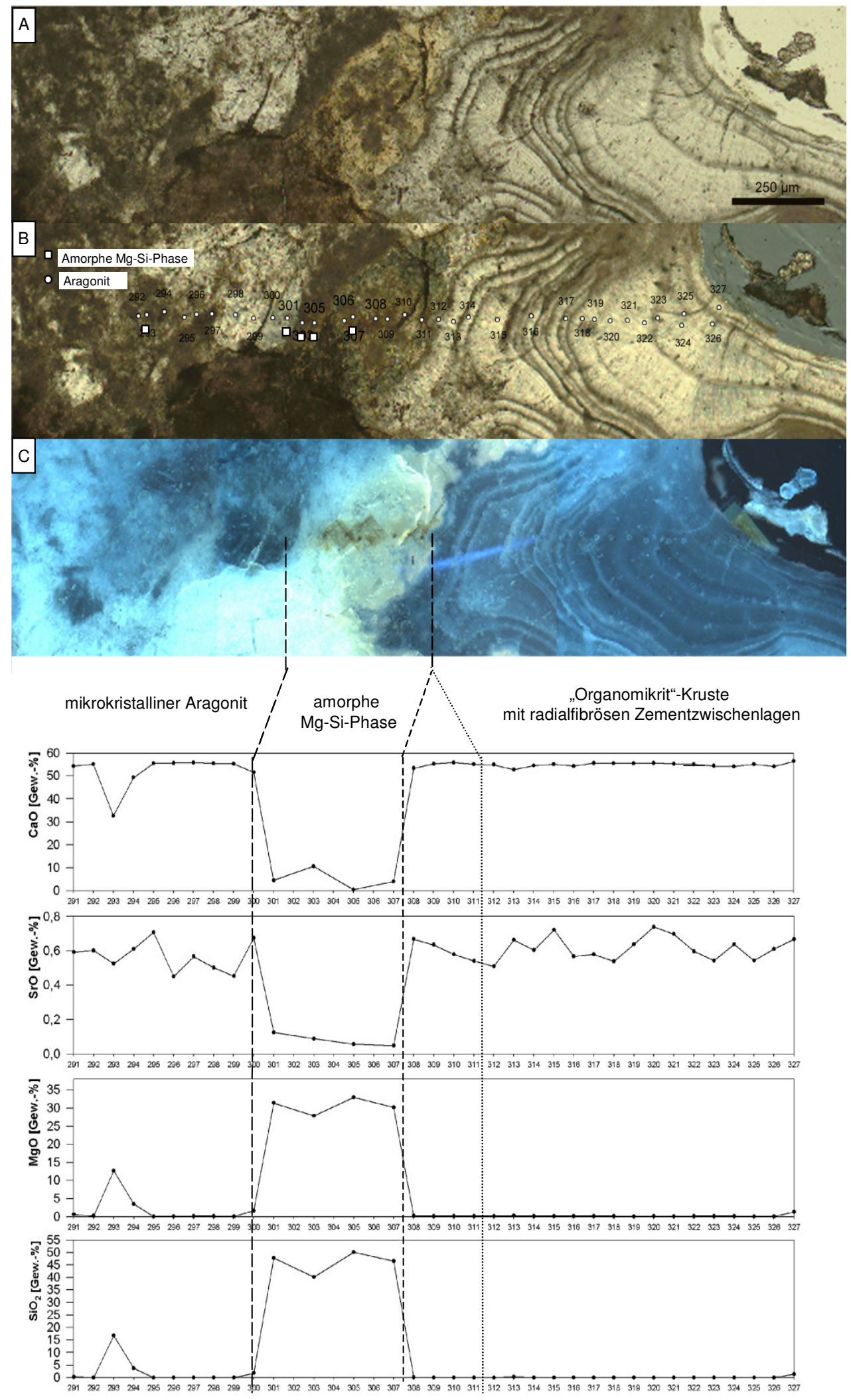

Abb. 5.7: Hauptelementverteilung. Probe Lake Thetis LTh 98/14, Line 5. A: HellfeldMikroskopaufnahme. B: Mikroskopaufnahme mit gekreuzten Polarisatoren. C: UV-FilterMikroskopaufnahme. Scyt: Scytonema-Filamente. Die Messpunkte 308-311 liegen optisch innerhalb der Mg-Si-Phase. Die Elementgehalte lassen allerdings auf eine im tieferen Bereich angeschossene Phase schließen, welche den „Organomikrit“-Lagen zugeordnet werden kann. 


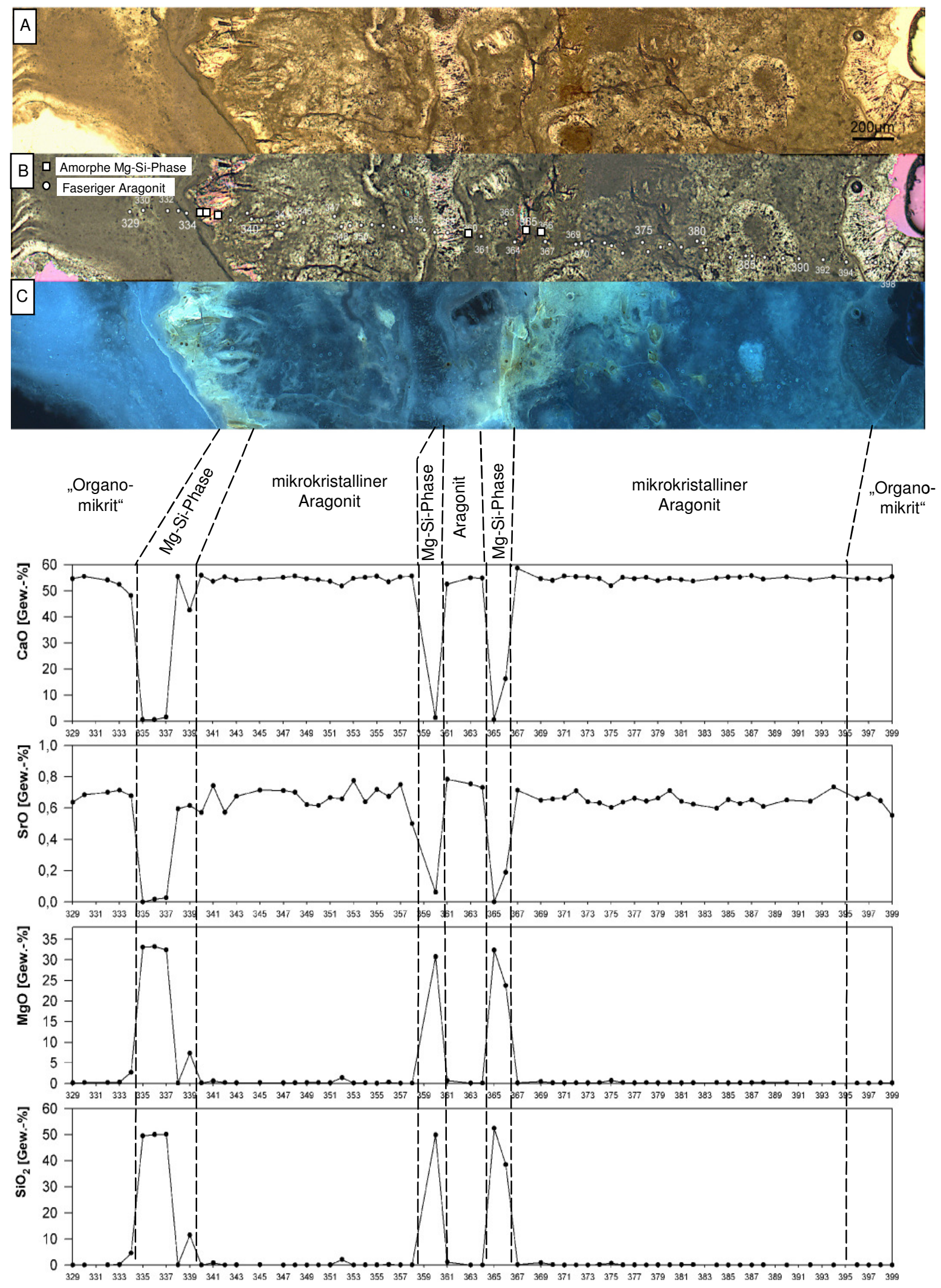

Abb. 5.8: Hauptelementverteilung. Probe Lake Thetis LTh 98/14, Line 6. A: HellfeldMikroskopaufnahme. B: Mikroskopaufnahme mit gekreuzten Polarisatoren. C: UV-FilterMikroskopaufnahme. 
Das $\mathrm{Ca} / \mathrm{Sr}$-Verhältnis (Gew.-\%) in den karbonatischen Probenanteilen beträgt - wie auch in den ICP-OES-Proben - meist zwischen 55 und 65. Hohe CaO-Gehalte von 50\% in einer MgSi-Phase (Line 1) zeigen an, dass es sich in diesem Fall um Mischsignale zweier Mineralphasen handelt. Das Mg/Si-Molverhältnis beträgt 1 : 1,25. Je deutlicher die Mg-SiPhase hervortritt und einzeln angeschossen werden kann, desto weniger verfälscht der $\mathrm{Mg}$ Anteil in den Karbonaten das Mg/Si-Molverhältnis (1:1,1 und $1: 1,25)$ (Abb. 5.4). In der reinen $\mathrm{Mg}$-Si-Phase verschiebt sich das Verhältnis zugunsten des Siliziums auf $1: 1,43$. Damit ist das $\mathrm{Mg} / \mathrm{Si}$-Molverhältnis im Vergleich zu dem $1: 1$-Verhältnis ähnlicher Mg-SiPhasen vom Satonda Crater Lake (ARP et al., 2003, 2004) und vom Great Salt Lake (Utah, USA, SPENCER et al., 1985) nur leicht verändert.

Die Mg-Si-Phase enthält neben einer leichten Anreicherung an $\mathrm{Na}$ (Tab. 5.1) auch Spuren von $\mathrm{Ca}, \mathrm{Sr}, \mathrm{Fe}$ und Mn und nach GREY et al. (1990) auch Spuren von Cl und S. Sie geht vermutlich auf die Lösung der Diatomeenschalen und einer Repräzipitation zurück (SPENCER et al., 1985; GREY et al., 1990).

SouZA-EGIPSY et al. (2005) beschreiben Mg-Si-Präzipitate vom Mono Lake (Kalifornien), deren Mg/Si-Verhältnis ca. 1 : 1,66 beträgt. Die Autoren konnten eine Zunahme im Si-Anteil von absterbenden Zellwänden zu fossilen Zellwänden beobachten. Die Mg-Si-Präzipitation ist an die Oberflächen der - hier allerdings - endolithischen Cyanobakterien und EPS gebunden und verläuft subsequent während der Zersetzung des Biofilms (,Post-mortem events“).

Die z.T. lagig ausgebildete Mg-Si-Phase tritt vorzugsweise im Übergang von den „Organomikrit“-Lagen zu den Aragoniten auf, wobei der Kontakt zu den Aragoniten unregelmäßig bis diffus ist. Der Kontakt zu den „Organomikriten“ dagegen ist von einer scharfen Grenzfläche geprägt. Ebenfalls aus der amorphen $\mathrm{Mg}$-Si-Phase bestehen die Scytonema-Filamente innerhalb der mikrokristallinen Aragonite. Rissfüllungen können ebenfalls diese Phase enthalten. Die hydrolysierte $\mathrm{Mg}$-Si-Phase weist charakteristische Schrumpfungsrisse auf.

\subsubsection{Thrombolith, Lake Clifton}

Der analysierte Bereich der thrombolithischen Probe vom Lake Clifton besteht aus einer nahezu reinen Mg-Si-Phase (Abb. 5.9). Nur ein Einzel-Messpunkt liefert Werte, wie sie für den Karbonatanteil charakteristisch sind. Die Gehalte an $\mathrm{CaO}$ liegen in der Mg-Si-Phase bei 0 Gew.-\% (Abb. 5.9), die SrO-Gehalte liegen ebenfalls an der Nachweisgrenze. Die MgO- und $\mathrm{SiO}_{2}$-Gehalte liegen bei 24 Gew.- $\% \mathrm{MgO}$ und $42 \mathrm{Gew.-} \% \mathrm{SiO}_{2}$. Das $\mathrm{Mg} / \mathrm{Si}-$ Verhältnis liegt mit $1: 1,66$ niedriger als das des Lake Thetis. 


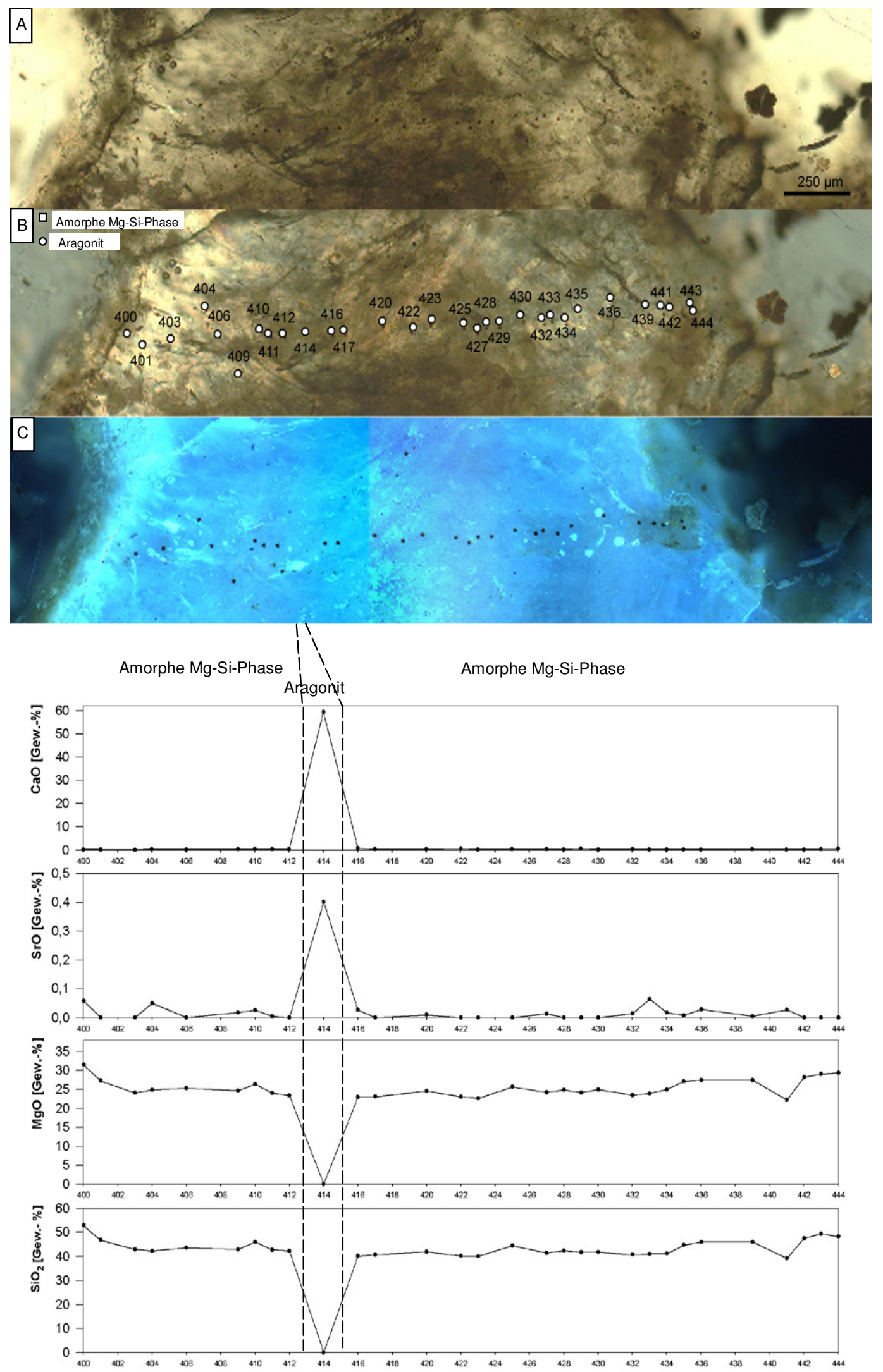

Abb. 5.9: Messung der Elektronenstrahlmikrosonde. Probe Lake Clifton LCl 02/7, Line 8. A: Hellfeld-Mikroskopaufnahme. B: Mikroskopaufnahme mit gekreuzten Polarisatoren. C: UVFilter-Mikroskopaufnahme. 


\subsection{Natrium-Karbonat-Aufschluss}

Um den Anteil der amorphen Mg-Si-Phase an der Massenbilanz zu ermitteln, wurden Natrium-Karbonat-Aufschlüsse zur Extraktion des Siliziums durchgeführt. An den untersuchten Proben wurde zudem der $\mathrm{C}_{\text {org }}$-Gehalt analysiert (Kap. 6.1).

Der Si-Anteil in den Mikrobialithen des Lake Thetis beträgt bis zu 1,23 Gew.-\%. Zusammen mit einem Mg-Anteil von ebenfalls 1 Gew.-\% und zusätzlichen Gehalten an Wasser ergeben sich für die Mg-Si-Phase zwischen 5-10 Gew.-\% (Tab. 5.4). Da der exakte Wasseranteil nicht bestimmt wurde, lässt sich keine genaue Stöchiometrie der Mg-Si-Phase ermitteln.

Sehr viel geringere Gehalte an Si liefern Proben, in denen die Mg-Si-Phase nicht vertreten ist. Die Stromatolithe der Shark Bay enthalten nur 0,07 und 0,25 Gew.-\% Si. Proben vom Lake Preston weisen einen Gehalt an Si von max. 0,25 Gew.-\% auf.

\begin{tabular}{ll|r}
\hline \multicolumn{2}{c|}{ Probe } & Si [Gew.-\%] \\
\hline \hline Lake Thetis & LTh 98/13 & $0,57-1,21$ \\
& LTh 98/14 & $0,39-1,57$ \\
& LTh 98/12 & $1,14-1,60$ \\
& LTh98/9 & 0,96 \\
& LTh 98/15 & $0,94-1,14$ \\
Lake Pollard & LPo 98/4 & 1,14 \\
Lake Preston & LPr 98/10 & 0,89 \\
& LPr 98/11 & 0,12 \\
& LPr 98/12 & 0,25 \\
Shark Bay & SB 98/18 & $0,14-0,17$ \\
& SB 98/29 & 0,25 \\
\hline
\end{tabular}

Tab. 5.4: Silizium-Gehalte der Mikrobialithe von Lake Thetis, Lake Preston, Lake Pollard und der Shark Bay. Der hohe Si-Anteil in den Stromatolithen vom Lake Thetis ist auf die autochthone, amorphe $\mathrm{Mg}$-Si-Phase zurückzuführen. In den Proben vom Lake Pollard geht der leicht erhöhte Gehalt auf die Siliziklasten zurück. 


\section{Biogeochemie}

\subsection{Kohlenstoff und Stickstoff}

Die Proben aus dem Lake Thetis zeigen ungewöhnlich hohe Anteile von bis zu 3,8 Gew.-\% $\mathrm{C}_{\text {org }}$ in der oberen Stromatolith-Kruste und bis zu 6,8 Gew.-\% $\mathrm{C}_{\text {org }}$ in den subfossilen Stromatolithen (Abb. 6.1; Tab. 6.1). Der aus dem Gesamt-Kohlenstoff berechnete KarbonatGehalt ist mit max. 82 Gew.-\% relativ gering, stimmt jedoch im Trend mit dem Ergebnis aus der Untersuchung der Elementverteilungen überein (vgl. Kap. 5.2). Zusätzlich zur Mg-SiPhase wird ein hoher Anteil an nicht näher bestimmbarer organischer Substanz für die nachweislich geringen Karbonat-Gehalte in einem röntgenographisch nahezu reinen Aragonit verantwortlich gemacht.

Bei detaillierter Beprobung einzelner Cyanobakterien-Lamellen (Probe LTh 98/13) zeigen sich kaum Schwankungen sowohl in den Kohlenstoff- als auch in den Stickstoff-Gehalten. Lediglich der Bereich unterhalb des „Organomikrit“-Überzuges am Top der Kruste, welcher hauptsächlich Filamentspuren von Scytonema sp. enthält (VIII in Abb. 6.1), weist einen minimal erhöhten $\mathrm{C}_{\text {org }}$-Gehalt und einen geringeren Gesamt-Karbonat-Gehalt bei gleichbleibendem N-Gehalt auf. Die basale Phormidium-Lamelle (I) und die oberste „Organomikrit“-Lage (IX) zeigen die geringsten N-Gehalte, wodurch sich in diesen Proben die höchsten C/N-Verhältnisse von 19,6 und 21,2 ergeben. Das höchste in den Lake ThetisProben ermittelte C/N-Verhältnis stammt aus der Caliche-Kruste und beträgt 38,8. Die Probe der subaquatischen Ebene des Lake Thetis enthält mit 0,4 Gew.-\% den höchsten Stickstoffgehalt. Alle weiteren Proben enthalten nicht mehr als 0,2 Gew.-\% N (Tab. 6.1).
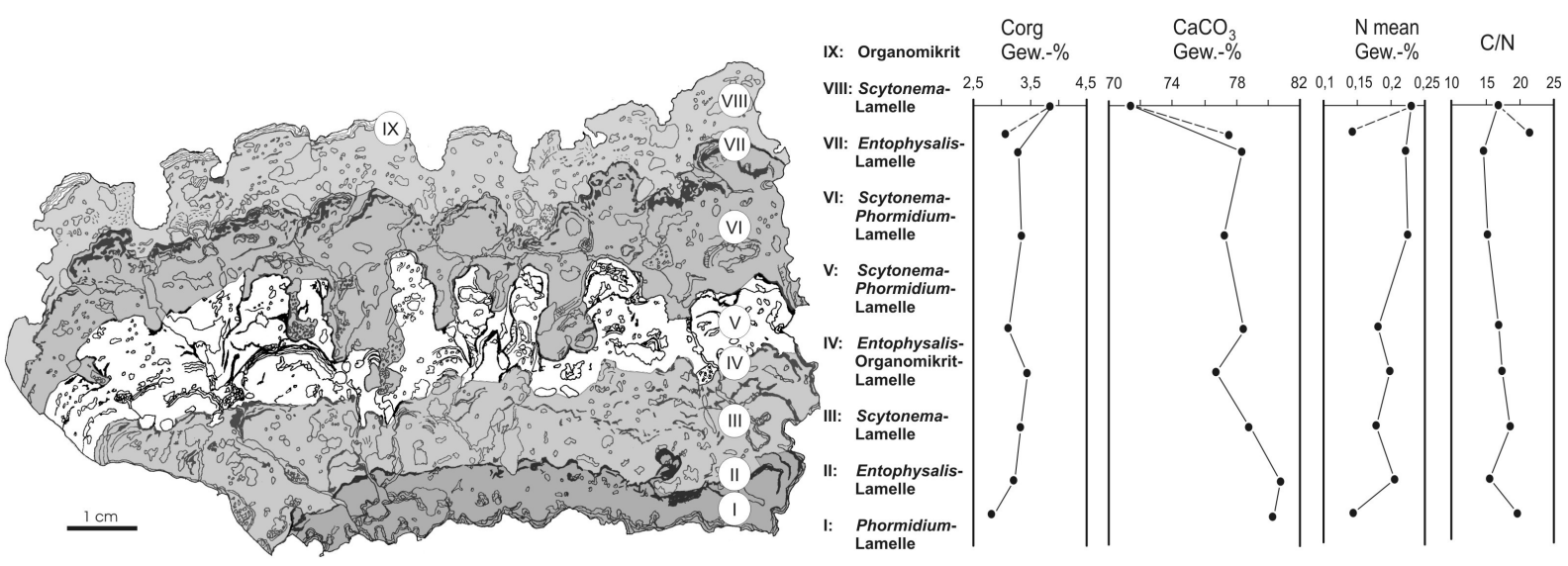

Abb. 6.1: Entnahmepunkte der Proben I bis IX einer laminierten Stromatolith-Kruste, Stromatolith-Typ 4 (Probe LTh 98/13). Die Gehalte an organischem Kohlenstoff und $\mathrm{CaCO}_{3}$ sowie die Stickstoff-Gehalte und die C/NVerhältnisse sind gegen die Lage im Profil aufgetragen (rechte Bildhälfte). 


\begin{tabular}{|c|c|c|c|c|c|c|c|}
\hline Probe & Material & $\begin{array}{c}\mathbf{C}_{\text {karb }} \\
{[\mathrm{Gew.- \% ]}}\end{array}$ & $\begin{array}{c}\mathrm{CaCO}_{3} \\
{[\mathrm{Gew} . \%]}\end{array}$ & $\begin{array}{c}\text { Corg }_{\text {org }} \\
\text { [Gew.-\%] }\end{array}$ & $\begin{array}{c}N_{\text {total }} \\
{[\text { Gew.-\%] }}\end{array}$ & $\begin{array}{c}\mathrm{C} / \mathrm{N}- \\
\text { Verhältnis }\end{array}$ & $\begin{array}{c}\mathbf{H}_{\text {total }} \\
{[\mathrm{Gew.- \% ]}}\end{array}$ \\
\hline \multicolumn{8}{|l|}{ Lake Thetis } \\
\hline LTh $98 / 12$ & subfossiler Stromatolith vom Uferbereich, äußere Kruste, Zemente & 8,9 & 74,0 & 3,2 & 0,11 & 30,5 & 0,4 \\
\hline LTh $98 / 12$ & subfossiler Stromatolith vom Uferbereich, äußere Kruste, Mikrit & 9,4 & 78,1 & 2,8 & 0,16 & 17,0 & 0,4 \\
\hline LTh $98 / 12.2$ & subfossiler Stromatolith vom Uferbereich,Gesamtgestein & 8,6 & 71,3 & 3,4 & 0,16 & 20,7 & 0,5 \\
\hline LTh 98/13 & $\begin{array}{l}\text { Stromatolith (äußere Kruste), zehnte Lage: abschließende weiße, } \\
\text { faserig-mikrokristalline Aragonitlamelle ("Organomikrit") }\end{array}$ & 9,3 & 77,4 & 3,0 & 0,14 & 21,2 & 0,5 \\
\hline LTh $98 / 13$ & $\begin{array}{l}\text { Stromatolith (äußere Kruste), zehnte Lage: dünne, braune bis grünlich- } \\
\text { schwarze, feinkristalline Aragonitlamelle mit Relikten kokkaler } \\
\text { Mikroorganismen (Entophysalis-Lage) }\end{array}$ & 8,6 & 71,3 & 3,9 & 0,23 & 16,8 & 0,5 \\
\hline LTh $98 / 13$ & $\begin{array}{l}\text { Stromatolith (äußere Kruste), sechste Lage: dünne, braune bis grünlich- } \\
\text { schwarze, feinkristalline Aragonitlamelle mit Relikten kokkaler } \\
\text { Mikroorganismen (Entophysalis-Lage+abschließende } \\
\text { Faseraragonitlage) }\end{array}$ & 9,4 & 78,2 & 3,3 & 0,22 & 14,6 & 0,6 \\
\hline LTh $98 / 13$ & $\begin{array}{l}\text { Stromatolith (äußere Kruste), achte Lage: braune bis grünlich- } \\
\text { schwarze, feinkristalline Aragonitlamelle mit Relikten kokkaler } \\
\text { Mikroorganismen (Entophysalis-Lage) }\end{array}$ & 9,3 & 77,3 & 3,4 & 0,22 & 15,1 & 0,5 \\
\hline LTh $98 / 13$ & $\begin{array}{l}\text { Stromatolith (äußere Kruste), vierte Lage: abschließende weiße, } \\
\text { faserige Aragonitlamelle ("Organomikrit") }\end{array}$ & 9,4 & 78,4 & 3,1 & 0,18 & 16,9 & 0,5 \\
\hline LTh $98 / 13$ & $\begin{array}{l}\text { Stromatolith (äußere Kruste), vierte Lage: braune bis grünlich-schwarze } \\
\text { feinkristalline Aragonitlamelle mit Relikten kokkaler Mikroorganismen } \\
\text { (Entophysalis-Lage) }\end{array}$ & 9,2 & 76,8 & 3,5 & 0,20 & 17,4 & 0,6 \\
\hline LTh $98 / 13$ & $\begin{array}{l}\text { Stromatolith (äußere Kruste), dritte Lage: weißgraue, feinfaserige } \\
\text { Aragonitlamelle mit erekten, dicken Filamenten (Scytonema-Lage) }\end{array}$ & 9,4 & 78,7 & 3,3 & 0,18 & 18,6 & 0,5 \\
\hline LTh $98 / 13$ & $\begin{array}{l}\text { Stromatolith (äußere Kruste), zehnte Lage: dünne braune bis grünlich- } \\
\text { schwarze, feinkristalline Aragonitlamelle mit Relikten kokkaler } \\
\text { Mikroorganismen (Entophysalis-Lage) }\end{array}$ & 9,7 & 80,8 & 3,2 & 0,21 & 15,6 & 0,6 \\
\hline LTh $98 / 13$ & $\begin{array}{l}\text { Stromatolith (äußere Kruste), erste Lage: weißgraue, feinfaserige } \\
\text { Aragonitlamelle mit wenigen dünnen erekten Filamentspuren } \\
\text { (Phormidium-Lage) }\end{array}$ & 9,6 & 80,3 & 2,8 & 0,14 & 19,6 & 0,4 \\
\hline LTh 98/14 & subfossiler Stromatolith, mittlere Kruste, $2 \mathrm{~cm}$ oberer Teil & 9,8 & 81,4 & 2,6 & 0,14 & 18,7 & 0,5 \\
\hline LTh 98/14 & subfossiler Stromatolith, mittlere Kruste, $2 \mathrm{~cm}$ mittlerer Teil & 9,9 & 82,2 & 2,3 & 0,12 & 19,1 & 0,3 \\
\hline LTh $98 / 14$ & subfossiler Stromatolith, mittlere Kruste, $2 \mathrm{~cm}$ unterer Teil & 9,0 & 75,0 & 2,6 & 0,14 & 19,0 & 0,5 \\
\hline LTh 98/15 & $\begin{array}{l}\text { subfossiler Stromatolith, subaquatische Ebene zwischen } \\
\text { Stromatolithstotzen, äußere grüne Kruste }\end{array}$ & 7,9 & 65,9 & 6,8 & 0,43 & 15,8 & 1,2 \\
\hline LTh $98 / 15$ & subfossiler Stromatolith, subaquatische Ebene, oberster $\mathrm{cm}$ & 9,5 & 78,8 & 3,2 & 0,21 & 15,4 & 0,6 \\
\hline LTh $98 / 15$ & subfossiler Stromatolith, subaquatische Ebene, unterer Teil & 9,2 & 77,1 & 3,1 & 0,14 & 21,5 & 0,5 \\
\hline LTh $98 / 16$ & subfossiler Stromatolith, mittlere Kruste, laminierter Top-Bereich & 8,9 & 73,8 & 4,6 & 0,20 & 23,1 & 0,7 \\
\hline LTh $98 / 16$ & subfossiler Stromatolith, mittlere Kruste, zementreicher Mittelteil & 8,8 & 73,6 & 3,1 & 0,16 & 19,4 & 0,5 \\
\hline LTh 98/09 & Karbonatische Bodenkruste (Caliche) & 7,5 & 62,3 & 3,9 & 0,10 & 38,8 & 0,4 \\
\hline \multicolumn{8}{|l|}{ Lake Pollard } \\
\hline LPo 98/04 & Stromatolith, Gesamtgestein & 8,4 & 70,3 & 3,8 & 0,16 & 23,3 & 0,5 \\
\hline \multicolumn{8}{|l|}{ Lake Preston } \\
\hline LPr 98/10 & fossiler Stromatolith, oberer Teil & 8,1 & 67,5 & 3,1 & 0,14 & 22,4 & 0,6 \\
\hline LPr $98 / 10$ & fossiler Stromatolith, unterer Teil & 8,2 & 68,6 & 1,8 & 0,03 & 68,2 & 0,1 \\
\hline LPr $98 / 11$ & Agglutinierter Stromatolith, Gesamtgestein & 8,6 & 71,3 & 1,4 & 0,05 & 25,5 & 0,2 \\
\hline LPr $98 / 12$ & fossiler Stromatolith, laminierter Teil & 8,3 & 69,0 & 1,7 & 0,05 & 38,3 & 0,2 \\
\hline LPr $98 / 12$ & fossiler Stromatolith, klastischer Teil & 9,0 & 75,3 & 1,6 & 0,03 & 64,2 & 0,1 \\
\hline \multicolumn{8}{|l|}{ Shark Bay } \\
\hline SB $98 / 18$ & fossiler Stromatolith & 8,9 & 74,0 & 2,2 & 0,04 & 60,8 & 0,2 \\
\hline SB $98 / 29$ & thrombolithische Aufwachsungen & 9,2 & 76,7 & 3,4 & 0,12 & 28,4 & 0,4 \\
\hline
\end{tabular}

Tab. 6.1: Ergebnisse der Kohlenstoff- und Stickstoffanalysen und der daraus errechneten $\mathrm{CaCO}_{3^{-}}$und $\mathrm{C}_{\mathrm{karb}}{ }^{-}$ Gehalte sowie das C/N-Verhältnis.

Die meteorisch überprägten Mikrobialithe des Lake Preston weisen mit 1,4 - 3,1 Gew.-\% $\mathrm{C}_{\text {org }}$ und 67,5 - 75,3 Gew.-\% Gesamtkarbonat geringere Gehalte als die Proben des Lake Thetis auf (Tab. 6.1). Fossile Proben der Hamelin-Pool-Stromatolithe enthalten 2,2 Gew.-\% $\mathrm{C}_{\text {org }}$ und 74 Gew.-\% $\mathrm{CaCO}_{3}$, rezente Anwachsungen enthalten 3,4 Gew.-\% $\mathrm{C}_{\text {org }}$ und 76,7 Gew.-\% $\mathrm{CaCO}_{3}$. Die $\mathrm{C}_{\text {org }}$-Gehalte aus Karbonaten des Lake Pollard betragen 3,8 Gew.-\% $\mathrm{C}_{\text {org }}$ und stimmen mit $\mathrm{C}_{\text {org }}$-Gehalten, wie sie auch die Lake Thetis-Proben zeigen, überein.

Die C/N-Verhältnisse in den Proben des Lake Preston und des Lake Pollard liegen zwischen 22,4 und 68,2. Ältere Probenbereiche der Lake Preston-Stromatolithe und fossile Stromatolith-Proben von der Shark Bay liefern die niedrigsten Stickstoffgehalte $(0,03$ - 0,04 Gew.-\%) und die größten C/N-Verhältnisse. 


\subsection{Biomarker}

\subsubsection{Subfossiler Stromatolith, Lake Thetis (LTh 98/14)}

Kohlenwasserstoffe: Als Hauptkomponenten sind $n$-Pentadecan und $n$-Heptadecan in der Probe enthalten (Abb. 6.2A). Die untersuchte Probe enthält zudem $n$-Alkene und mittkettig verzweigte Alkane.

Interpretation: $n$-Pentadecan $\left(\mathrm{C}_{15}\right), n$-Heptadecan $\left(\mathrm{C}_{17}\right)$ und $n$-Nonadecan $\left(\mathrm{C}_{19}\right)$ stammen aus Cyanobakterien (ZeNG et al. 1992b; HefTER, 1997; ThIEL et al., 1997) und Algen (GelPI et al. 1970; TiSsot \& Welte, 1984). Die mittkettig verzweigten Alkane gelten als spezifische Biomarker für Cyanobakterien (SHIEA et al., 1990).

Fettsäuren: In der Fettsäurefraktion ist eine Cyclopropylsäure die Hauptkomponente (Abb. 6.2B, C). Eine homologe Folge $n$-geradzahliger Fettsäuren zeigt eine bimodale Verteilung mit zwei Maxima bei $n$-Hexadecansäure $\left(\mathrm{C}_{16}\right)$ und n-Tetracosansäure $\left(\mathrm{C}_{24}\right)$. Weitere Komponenten sind iso-/anteiso-Alkansäuren, n-Alkensäuren sowie mittkettig verzweigte Alkansäuren. Darüber hinaus konnte Vaccensäure $(18: 1 \omega 7 \mathrm{c})$ identifiziert werden. Eine $17 \beta(\mathrm{H}), 21 \beta(\mathrm{H})$-Bishomohopansäure $\left(\beta \beta \mathrm{C}_{32}\right)$ ist ebenfalls in hoher Konzentration enthalten.

Interpretation: Die $\mathrm{C}_{19}$ Cyclopropylsäure ist häufig in anaeroben Bakterien vertreten (vgl. FANG \& BARCELONA, 1998). Ihre Gegenwart in der Stromatolith-Probe kann möglicherweise auf Einträge durch aus dem Seebecken eingeschwemmte SPB zurückgeführt werden. Auch

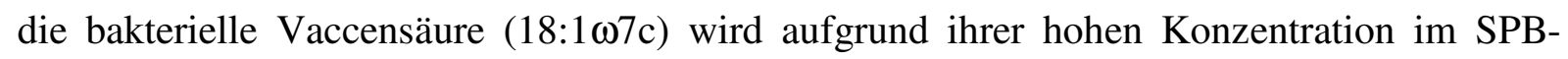
reichen Seebodensediment dieser Quelle zugeschrieben (vgl. Kap. 6.2.3, Abb. 6.4). Die 17ß(H),21ß(H)-Bishomohopansäure ist ein Degradationsprodukt der Bakteriohopanpolyole (DoBSON et al., 1988). Als Quelle für die iso-/anteiso-Alkansäuren werden anaerobe Bakterien, vor allem Sulfatreduzierer, vermutet. Iso-/anteiso-Alkansäuren sind u.a. aus dem weitverbreiteten, Sulfatreduzierender Desulfovibrio desulfuricans bekannt (Boon et al., 1977). 

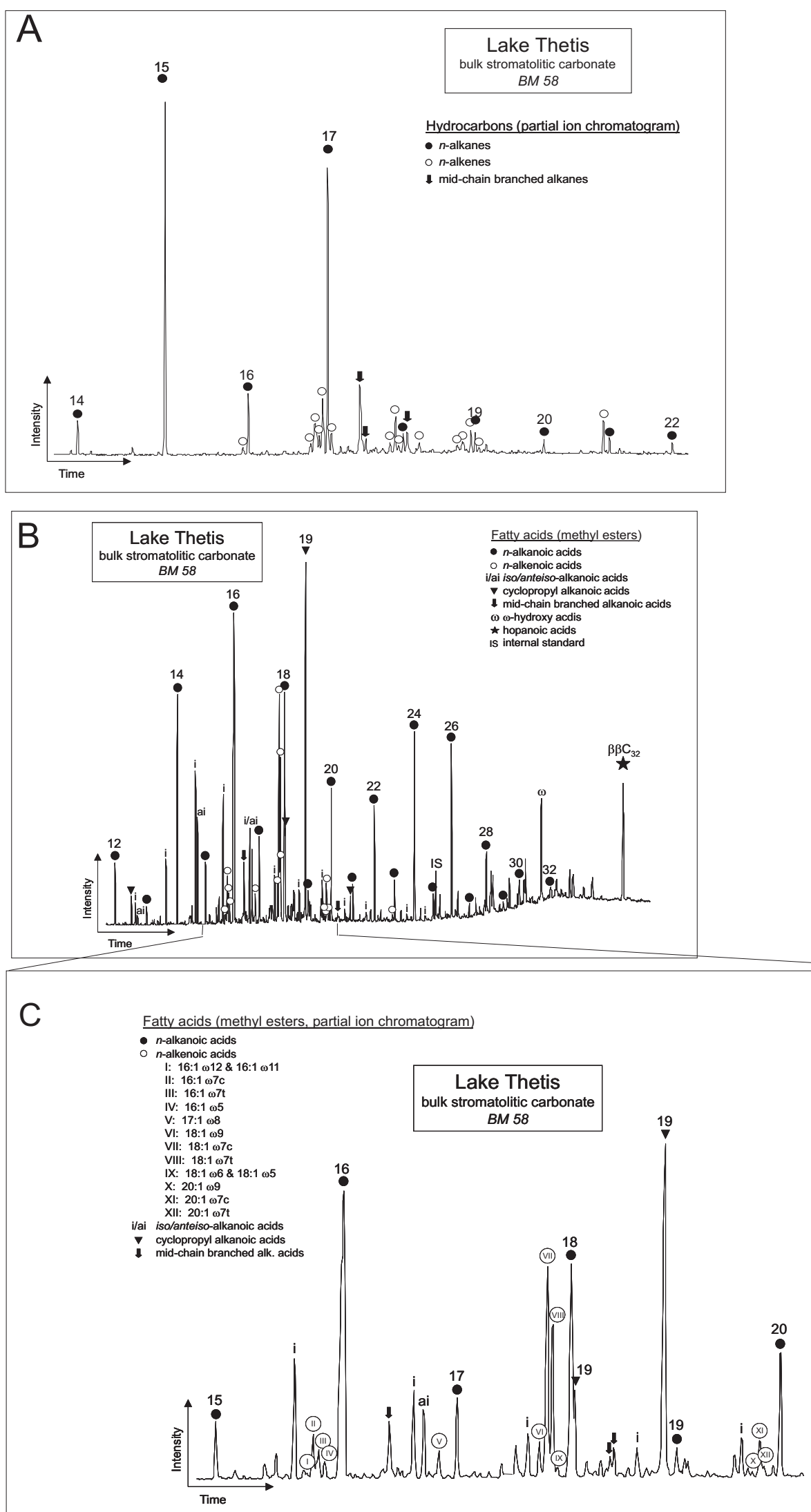

Abb. 6.2: Biomarker aus einer stromatolithischen Karbonatkruste vom Lake Thetis. A: Totalionenstromchromatogramm der Kohlenwasserstoff-Fraktion. B: Totalionenstromchromatogramm der Fettsäure-Fraktion (als Methylester). C: Teilausschnitt des Chromatogramms B. 


\subsubsection{Subfossiler Stromatolith, „Organomikrit“"-Lage, Lake Thetis (LTh 98/13)}

Fettsäuren: In der obersten „Organomikrit“-Lage ist die Hauptkomponente die nDocosansäure $\left(\mathrm{C}_{22}\right)$ (Abb. 6.3). Die Probe enthält zudem iso-/anteiso-Alkansäuren, eine $\mathrm{C}_{19^{-}}$

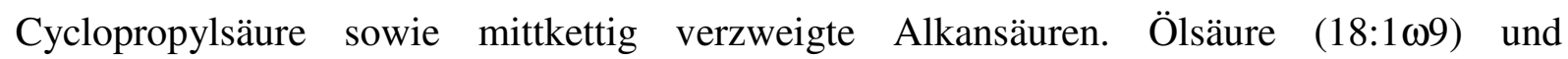

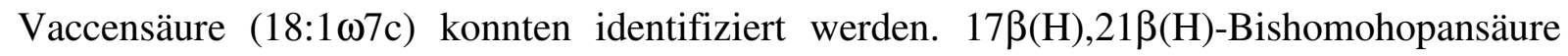
$\left(\beta \beta C_{32}\right)$ ist ebenfalls, wenn gleich in geringer Konzentration, vertreten.

Interpretation: Das Auftreten von $n$-Docosansäure $\left(\mathrm{C}_{22}\right)$ als Hauptkomponente in natürlichen Proben ist äußerst ungewöhnlich. Spezifische Quellen für diese Verbindungen wurden bislang nicht identifiziert. Die Herkunft dieser Verbindung in der Stromatolith-Probe aus dem Lake Thetis kann daher nicht näher eingegrenzt werden. Die Hopansäure entstammt einem bakteriellen bzw. cyanobakteriellen Biosynthese-Pfad (OuRISSON et al., 1997; DoBSON et al., 1988). Iso-/anteiso-Alkansäuren, die als Hinweis auf Sulfatreduzierer gewertet werden können (BOON et al., 1977; EDLUND et al., 1985; WANG et al., 1998), treten nur untergeordnet auf.

\subsubsection{SPB-reiches Seebodensediment, Lake Thetis (LTh 02/20)}

Fettsäuren: Die Hauptkomponente ist die Vaccensäure (18:1 107c, Abb. 6.4A, B). Zudem sind in der Probe weitere $n$-Alkensäuren, Cyclopropylsäuren, mittkettig verzweigte Alkansäuren und Hopansäuren enthalten.

Interpretation: Die Probe besteht aus Aragonit-Präzipitaten, Diatomeen, Ostrakoden-Schill und einem hohen Anteil an phototrophen, anoxygenen SPB. Die Vaccensäure wird über einen anaeroben Pfad synthetisiert (BoBBIE \& WHITE, 1980; WHITE et al., 1983) und ist vor allem aus anaeroben Bakterien bekannt (FANG \& BARCELONA, 1998). Die Cyclopropyl-Alkansäure wird als bakterielle Fettsäure einer anaerob lebenden Population interpretiert (FANG \& BARCELONA, 1998) und wird ebenfalls der Quelle der SPB zugeschrieben. Die Steroide entstammen überwiegend pflanzlichen Quellen und gehen vermutlich auf Einträge aus der Vegetation im Uferbereich des Sees zurück. Bakteriellen Ursprungs ist die $17 \beta(H), 21 \beta(H)$ Bishomohopansäure $\left(\beta \beta \mathrm{C}_{32}\right)$ (vgl. DoBSON et al. 1988), deren geologische Variante $\left(17 \alpha(\mathrm{H}), 21 \beta(\mathrm{H})\right.$-Bishomohopansäure $\left.\left(\alpha \beta \mathrm{C}_{32}\right)\right)$ in sehr geringer Konzentration ebenfalls in der Probe enthalten ist. 


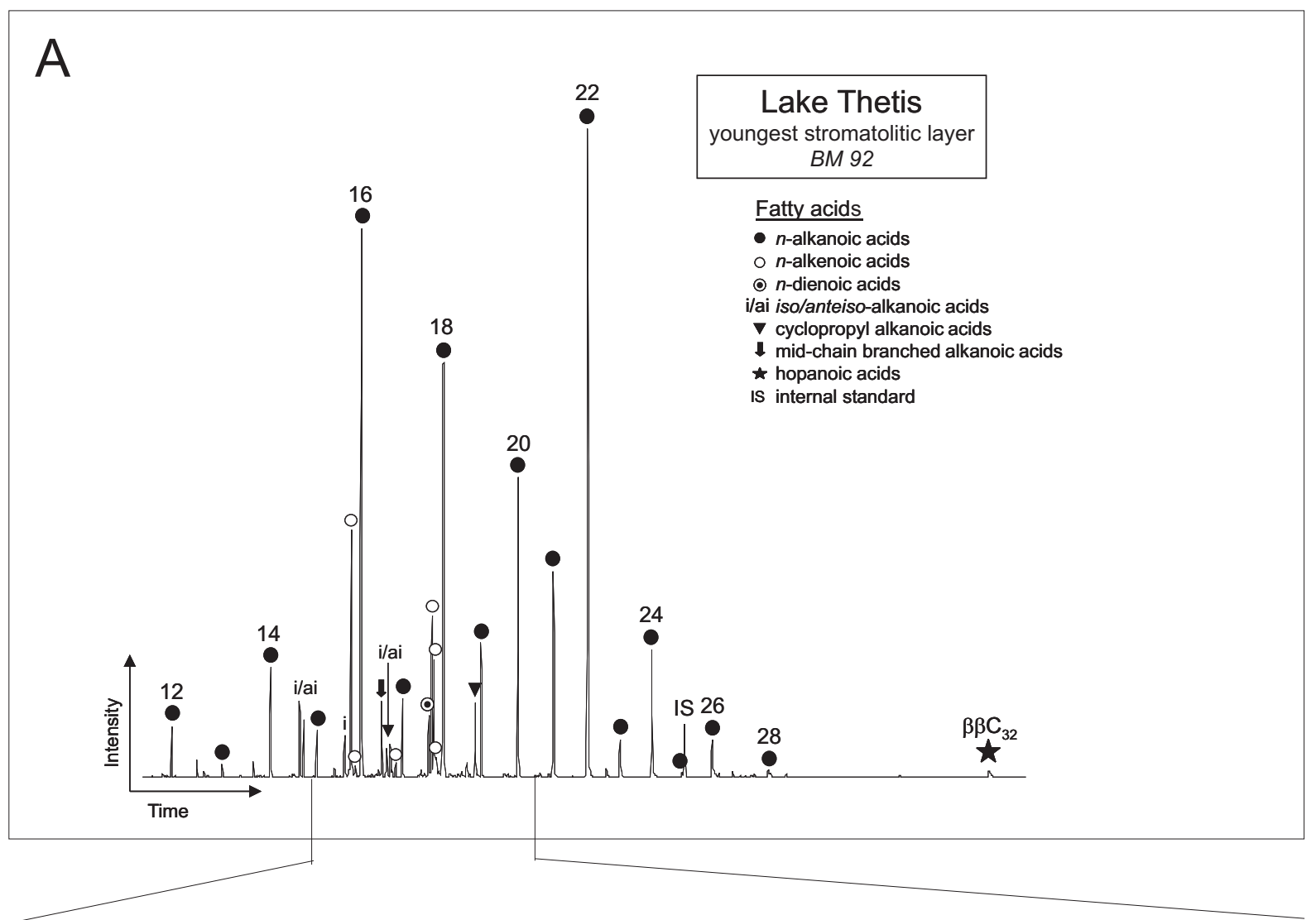

B

Fatty acids (partial ion chromatogram)

- $n$-alkanoic acids

O $n$-alkenoic acids
I: $16: 1 \omega 7 \mathrm{c}$
II: $16: 1 \omega 5$
III: $17: 1 \omega 8$
IV: $18: 1 \omega 9$
$\mathrm{V}: 18: 1 \omega 7 \mathrm{c}$
VI: $18: 1 \omega 7 \mathrm{t}$
$n$-alkadieonic acid
VII: $18: 2 \omega 6,9$

i/ai iso/anteiso-alkanoic acids

$\nabla$ cyclopropyl alkanoic acids

$\downarrow$ mid-chain branched alk. acids

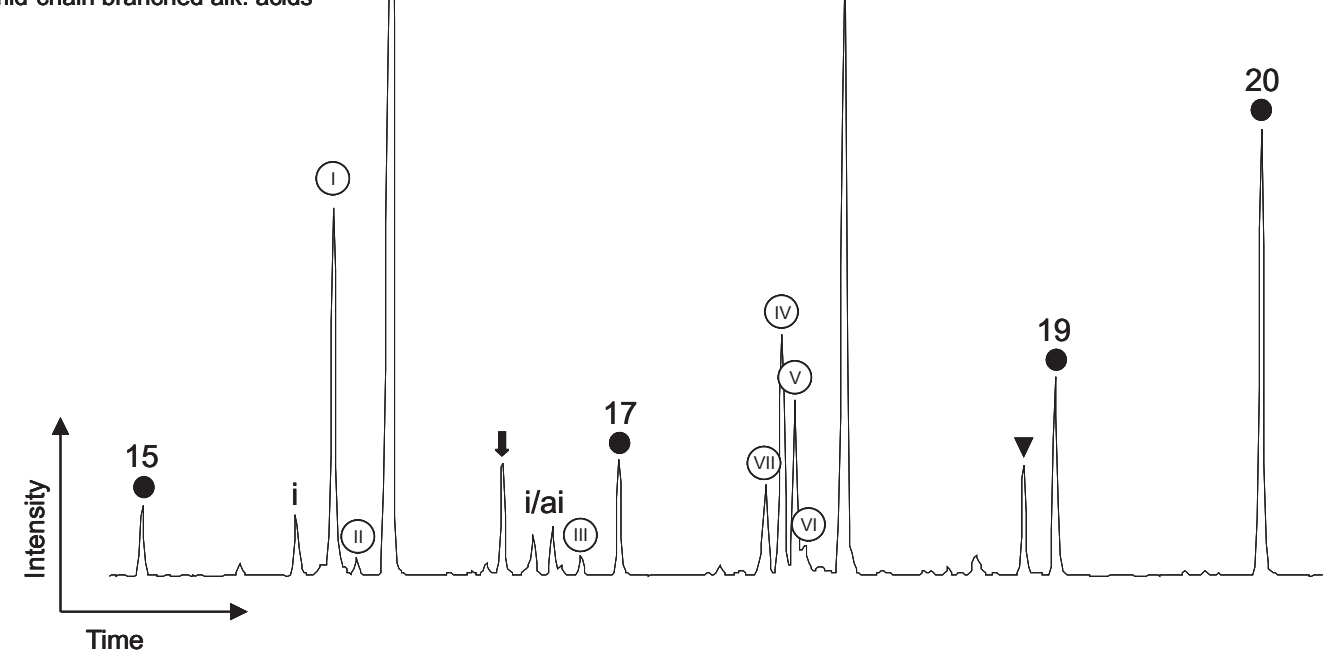

Abb. 6.3: Biomarker aus der obersten „Organomikrit“-Lage der stromatolithischen Karbonatkruste vom Lake Thetis. A: Totalionenstromchromatogramm der Fettsäuren (als Methylester). B: Teilausschnitt des Chromatogramms B. 


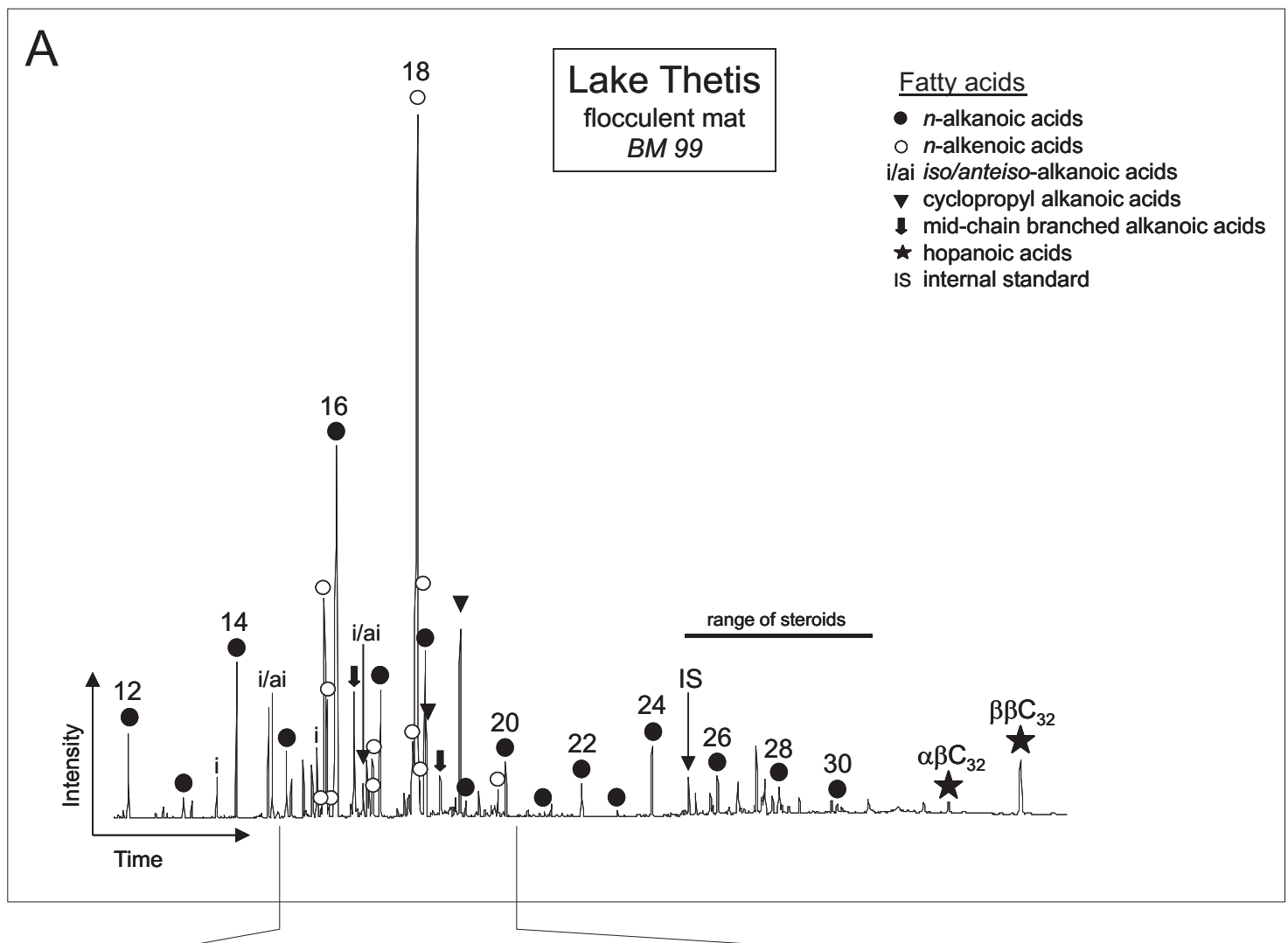

B

Fatty acids (partial ion chromatogram)

- $n$-alkanoic acids

- $n$-alkenoic acids

I: $16: 1 \omega 9$

II: $16: 1 \omega 7 \mathrm{c}$

III: $16: 1 \omega 7 \mathrm{t}$

IV: $16: 1 \omega 5$

V: $17: 1 \omega 8$

VI: $17: 1 \omega 6$

VII: $18: 1 \omega 9$

VIII: $18: 1 \omega 7 c$

IX: $18: 1 \omega 7 \mathrm{t}$

$\mathrm{X}: 18: 1 \omega 5$

XI: $20: 1 \omega 7$

$\mathrm{i} /$ ai iso/anteiso-alkanoic acids

$\nabla$ cyclopropyl alkanoic acids

1 mid-chain branched alk. acids

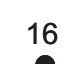

mid-chain branched alk. acids

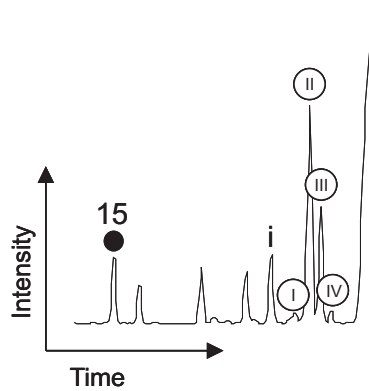

(1)

(III)

Lake Thetis

SPB-rich sediment

"flocculent mat" BM 99
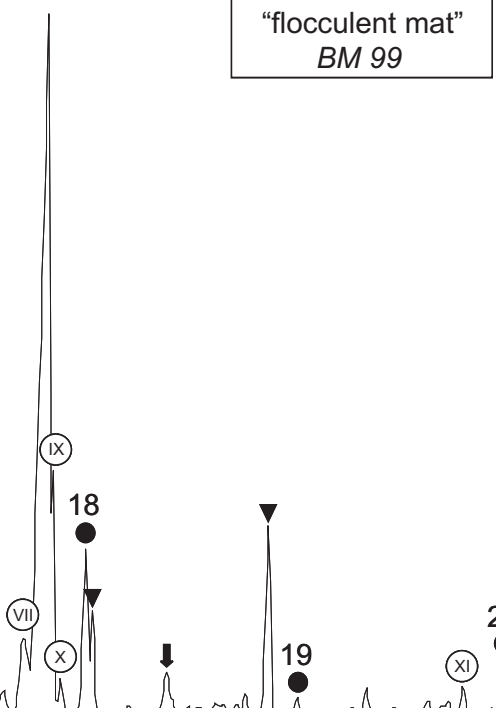

19

20

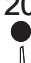

Abb. 6.4: Biomarker aus dem Schwefelpurpurbakterien-reichen Sediment des Lake Thetis. A: Totalionenstromchromatogramm der Fettsäure-Fraktion (als Methylester). B: Teilausschnitt des Chromatogramms A. 


\subsubsection{Thrombolith-Kruste, Lake Clifton (LCL 02/05)}

Kohlenwasserstoffe: Die Hauptbestandteile der Kohlenwasserstoff-Fraktion sind einfach und zweifach ungesättigte, hochverzweigte Isoprenoide (HBI 25/2 und HBI 25/1, Abb. 6.5A), $n$-Alkane (n-Pentadecan, $n$-Heptadecan), mittkettig verzweigte Methylheptadecane, Hopene sowie Diplopten.

Interpretation: HBI sind charakteristische Inhaltstoffe von Diatomeen (NICHOLS et al., 1988; Rowland \& RoBSON, 1990; VolKMAn et al., 1994). Ungesättigte C $_{25}$ HBI (HBI 25/2 und HBI 25/1) sind Hauptkomponenten der Kohlenwasserstoff-Fraktion fossiler, erosiv eingeebneter Mikrobialithe des Lake Clifton (THIEL, 1997). Ebenso wie bei den Stromatolithen des Lake Thetis werden $n$-Pentadecan und $n$-Heptadecan als Marker für Cyanobakterien und Algen interpretiert. Methylheptadecane markieren Einträge aus Cyanobakterien (vgl. SHIEA et al., 1990; ZENG et al., 1992b). Diplopten, ein Vorläufermolekül des ebenfalls in der Probe enthaltenen Hop-17(21)-en, wurde häufig in Cyanobakterien (ROHMER et al., 1984), aber auch in anderen Bakterien beobachtet (OURISSON et al., 1979; ROHMER, et al., 1984). Besonders die $2 \beta$-Methylhopanoide gelten als spezifische Biomarker für Cyanobakterien (SumMONS et al., 1999). In SPB wurden Hopanoide bislang nicht nachgewiesen (ROHMER, et al., 1984).

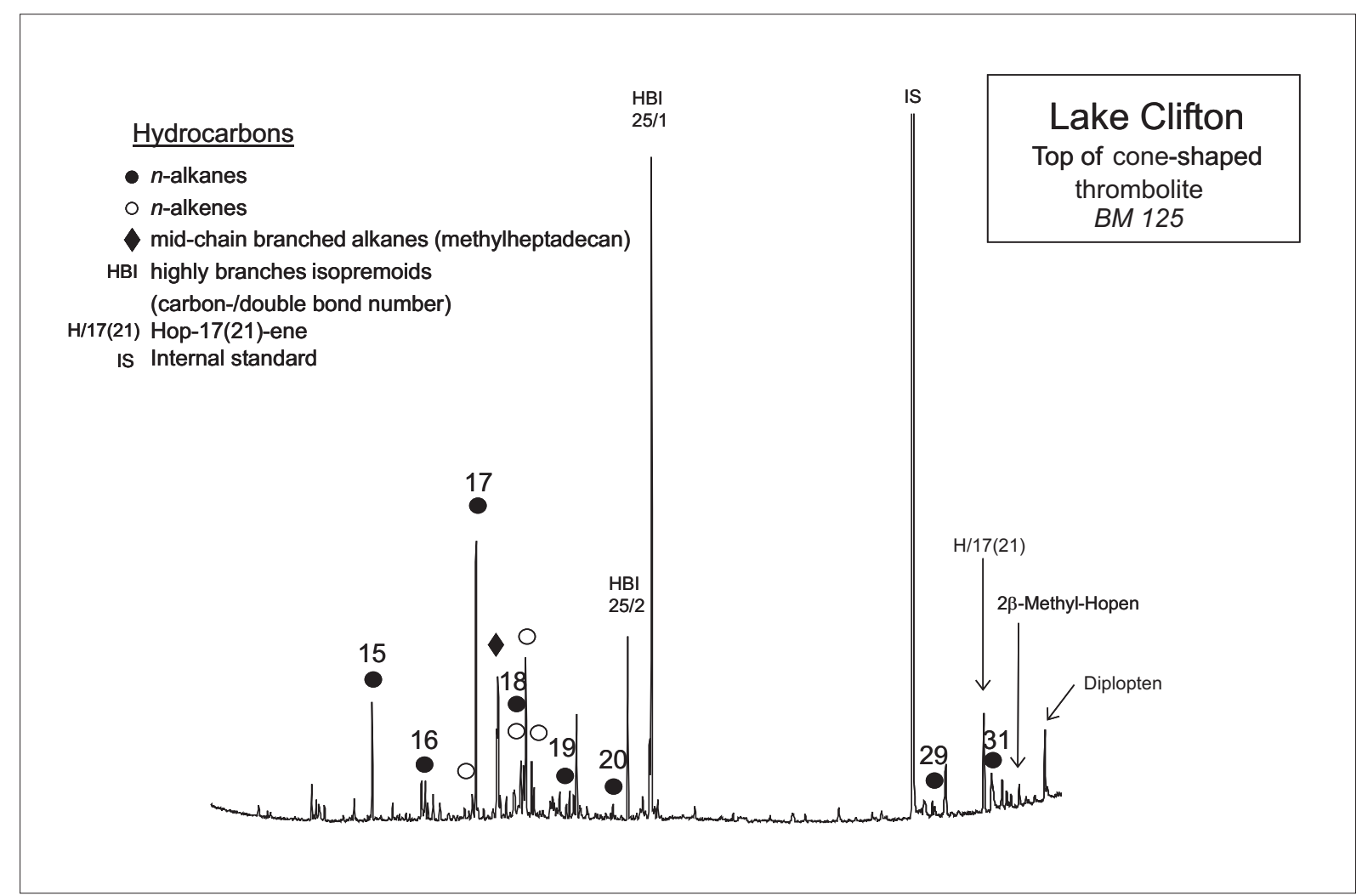

Abb. 6.5: Biomarker aus einem thrombolithischen Mikrobialith des Lake Clifton (LCl 02/5). Totalionenstromchromatogramm der Kohlenwasserstoffe. 


\section{Hydrochemie}

Die westaustralischen Salzseen und die Shark Bay wurden während der Feldkampagne 1998 in der Regenzeit (Seewasserhochstand) und während der Feldkampagne 2002 am Ende der Trockenzeit (Seewassertiefstand) beprobt.

Am Lake Thetis markiert ein ca. $15 \mathrm{~m}$ breiter Saum aus Seegräsern den Uferbereich bei Seespiegelhochstand. Die Seespiegelschwankung zwischen Winter- und Sommermonaten beträgt max. 0,5 m. Während des Seespiegeltiefstandes ist der Uferbereich dreigeteilt. In der äußersten Zone befinden sich erodierte, eingeebnete Stromatolithe, welche trocken liegen. Daran schließt sich eine Zone mit Biohermen an, die vom Wasser umgeben sind, allerdings trockene Stromatolith-Kappen aufweisen. In Richtung des flach abfallenden Seebeckens befindet sich eine dritte Zone mit vollständig vom Wasser bedeckten Biohermen.

Das Vorkommen der Bioherme am Lake Clifton konzentriert sich auf das E-Ufer. Ein $20 \mathrm{~m}$ breiter Streifen eingeebneter, erodierter Bioherme mit konzentrischem Aufbau liegt bei Seespiegeltiefstand frei, bei Seespiegelhochstand ist er dagegen von Wasser bedeckt. Unter permanenter Wasserbedeckung liegen die kugel- bis kegelförmigen thrombolithischen Bioherme. Für den Lake Clifton wird am E-Ufer der Zutritt eines Frischwasseraquifers angenommen (vgl. MOORE, 1987; MOORE \& BURNE, 1994).

Lake Preston zeigt ebenfalls starke Seespiegelschwankungen während der klimatischen Wechsel. Der N- und der höher gelegene S-Teil des Sees besitzen bei Seespiegelhochstand eine Verbindung zueinander. Bei Seespiegeltiefstand trocknet der N-Teil komplett aus, während die Ausdehnung des S-Teils so stark reduziert ist, dass ein ca. $500 \mathrm{~m}$ breiter, von Karbonatschlamm bedeckter Ufersaum trocken liegt.

Der Lake Walyungup konnte nur im Zustand des Seespiegeltiefstandes beobachtet werden. Entlang des ca. $300 \mathrm{~m}$ breiten Ufersaums ragen fossile Mikrobialithe in Form von max. 2 x 0,8 x 0,5 m großen Biohermen heraus. Direkt an der Wasserlinie befinden sich weiße, hochporöse Karbonatkrusten.

Aufgrund der Verbindung zum Indischen Ozean unterliegt die Shark Bay hinsichtlich des Wasserstandes nicht jahreszeitlichen Schwankungen, sondern dem tidalen Zyklus, welcher die Wasserbedeckung der intertidalen Stromatolithe steuert. 


\subsection{Hydrochemische Daten}

Infolge der starken trockenzeitlichen Evaporation und der Verdünnung der Wasserkörper während der Regenzeit unterliegen die Seen ausgeprägten Salinitätsschwankungen. Lake Thetis hatte gegenüber dem Meerwasser (35 g/Kg) sowohl in der Regenzeit (RZ) mit $37 \mathrm{~g} / \mathrm{Kg}$ als auch in der Trockenzeit (TZ) mit $47 \mathrm{~g} / \mathrm{Kg}$ höhere Salinitäten.

Die Salinität des Seewassers in Lake Clifton betrug bei Seespiegelhochstand $30 \mathrm{~g} / \mathrm{Kg}$ und bei niedrigem Seespiegel $68 \mathrm{~g} / \mathrm{Kg}$ und ist somit höher als von MOORE (1987) dokumentiert. Messungen während eines kompletten saisonalen Zyklus ergaben 17-30 g/Kg (MOORE, 1979 in Moore et al., 1983). Eine Steigerung der Sommer-Salinität im Lake Clifton ist bereits seit mehreren Jahren bekannt: von 29,8 g/Kg (MOORE et al., 1983) und $35 \mathrm{~g} / \mathrm{Kg}$ (MoORE \& BuRne, 1994) zu 48 g/Kg (KonISHI et al., 2001). Die zunehmende Salinität des Seewassers im Lake Clifton kann durch eine vermehrte Frischwasser-Entnahme hervorgerufen werden, wodurch das Grundwasser verstärkt aus dem östlich gelegenen, marinen Ästuar Peel Inlet gespeist wird.

Lake Prestons S-Arm zeigt die höchsten Salinitäten, sowohl zur Regen- (90 g/Kg) als auch zur Trockenzeit (199 g/Kg), durch evaporative Aufkonzentration. Im Vergleich dazu war die Salinität des N-Arms und des Überlaufs mit $9 \mathrm{~g} / \mathrm{Kg}$ weitaus geringer.

Lake Pollard (28 g/Kg) und Lake Walyungup (22 g/Kg) wiesen geringere Salinitäten als das Meerwasser auf.

In der Shark Bay waren die Salinitäten gegenüber dem Meerwasser erhöht, was durch eine Evaporations-Folge vom stark marin-beeinflussten Messpunkt bei Monkey Mia (45 g/Kg) hin zum lagunär-geprägten Beprobungsstandort bei Hamelin Pool (RZ: 64 g/Kg; TZ: 70 g/Kg) verdeutlicht wird (Abb. 7.1).

In Bezug auf ihre Ionenverhältnisse sind die Salzseen äquivalent zum Meerwasser (Nordstrom et al., 1979). Durch die klimatischen Bedingungen unterliegen sie allerdings stark evaporativen Prozessen. Durch eine Eindunstung und Wiederauffüllung durch meteorische Wässer und Zuflüsse, deren Ionenfracht sehr gering ist, bleiben die Ionenverhältnisse nahezu unverändert, wodurch sich beim Vergleich zweier sich konservativ verhaltenen Ionen eine Regressionsgerade ergibt (Abb. 7.1).

Es handelt sich bei den Seen um Na-Cl-dominierte Salzseen. Der Lake Clifton unterscheidet sich in der relativen Ionenhäufigkeit durch eine Prävalenz des $\mathrm{Ca}^{2+}$ gegenüber dem $\mathrm{K}^{+}$von den übrigen Seen, welche sich durch eine Ionenhäufigkeit von $\mathrm{Cl}^{-}>\mathrm{Na}^{+}>\mathrm{Mg}^{2+}>\mathrm{SO}_{4}{ }^{2-}>\mathrm{K}^{+}$ $>\mathrm{Ca}^{2+}$ auszeichnen (Tab. 7.1, Anhang 7). In der Shark Bay zeigen marine Milieus $\mathrm{Ca}^{2+} \geq \mathrm{K}^{+}$ und lagunäre Milieus $\mathrm{K}^{+} \geq \mathrm{Ca}^{2+}$. 


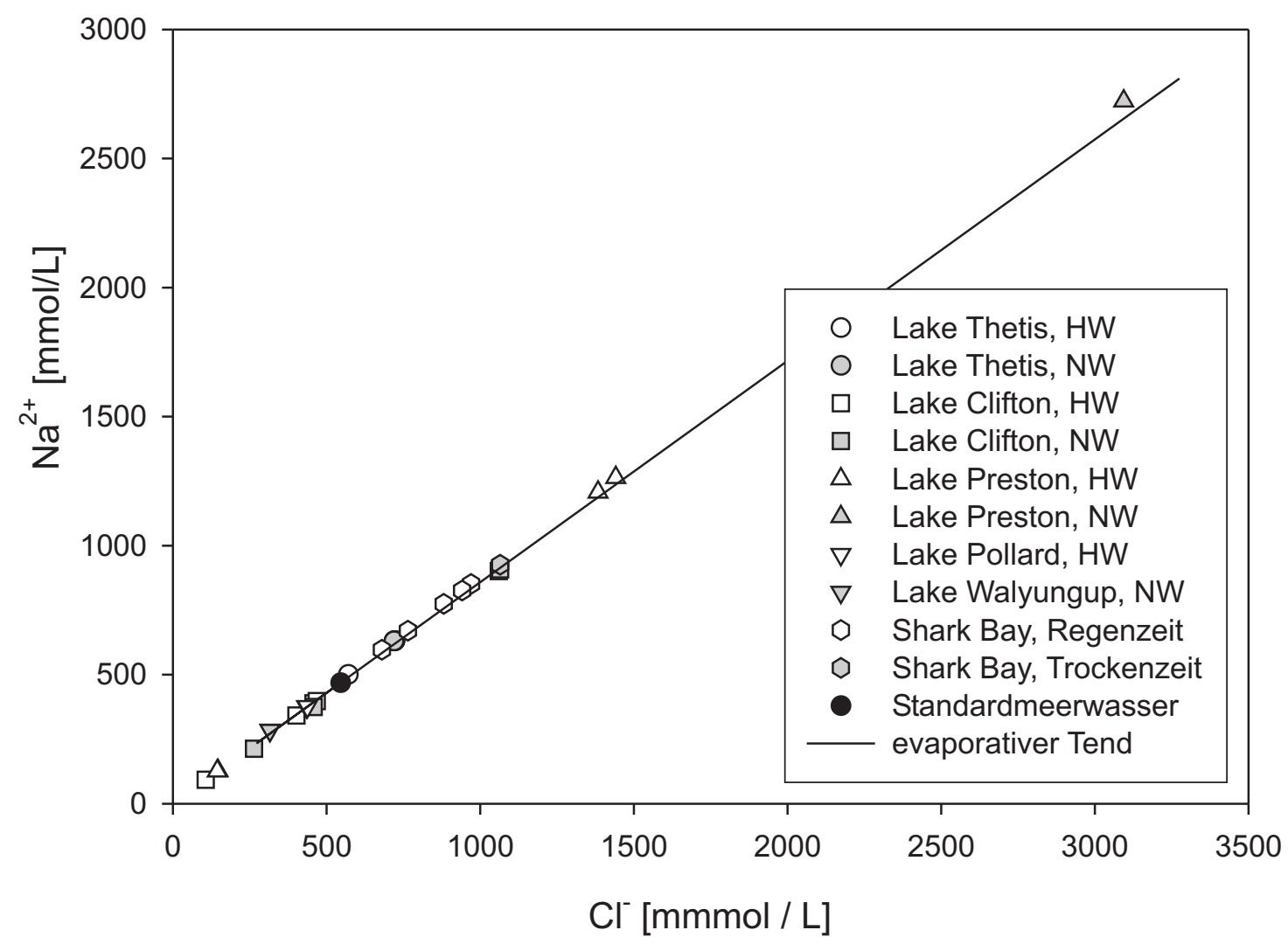

Abb. 7.1: Die fünf Seen und die Shark Bay zeigen einen deutlich evaporativen Trend bezüglich der sich konservativ verhaltenden Ionen.

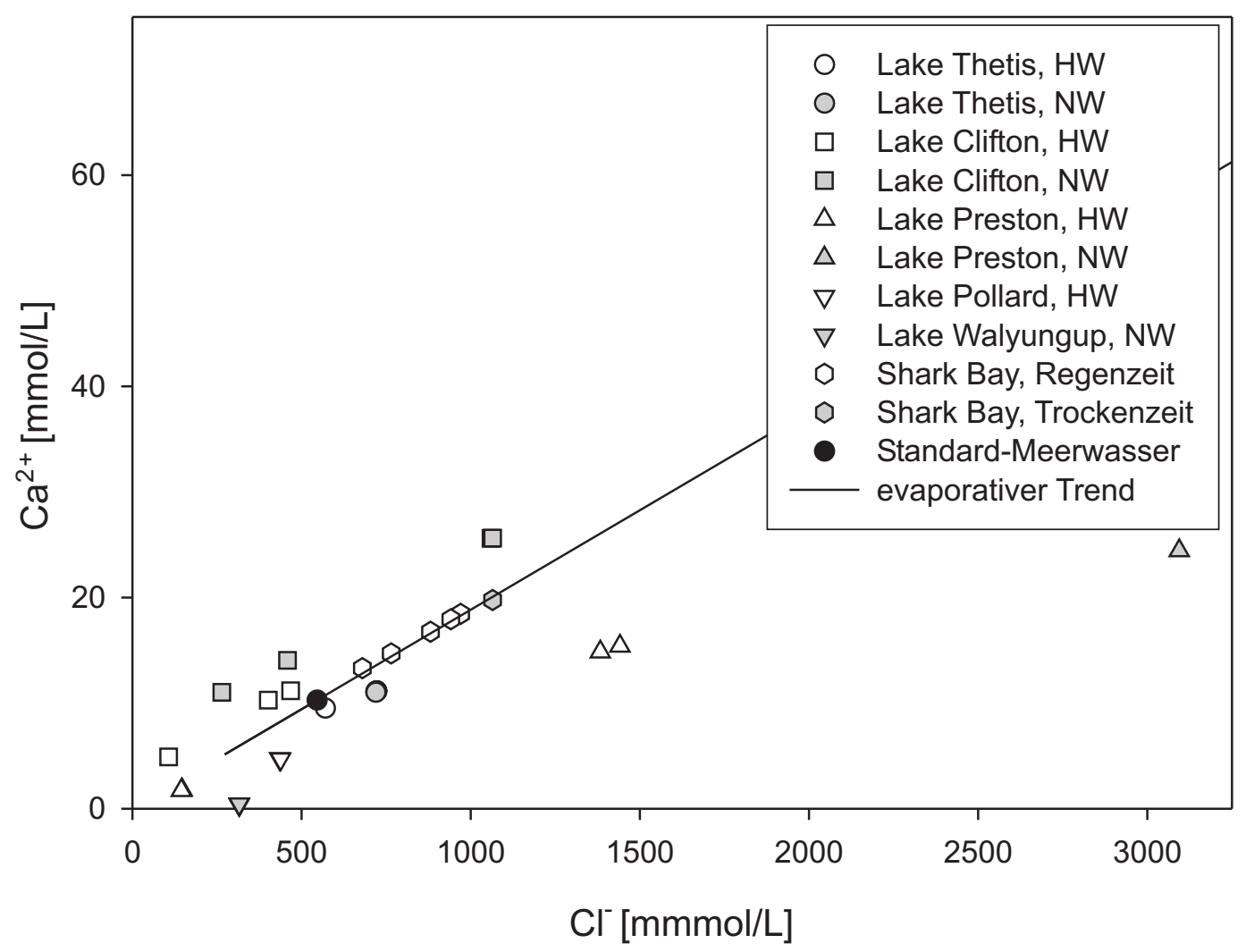

Abb. 7.2: Der evaporative Trend verdeutlicht durch Abweichungen zum einen erhöhte Kalzium-Gehalte (Lake Clifton) und zum anderen im Verhältnis zu niedrige Gehalte (Lake Thetis). In der Shark Bay unterliegt auch das Ca einer evaporativen Anreicherung. 


\begin{tabular}{|l|l|}
\hline Salzseen \& Shark Bay & relative lonenhäufigkeit (mol) \\
\hline Lake Thetis & $\mathrm{Cl}^{-}>\mathrm{Na}^{+}>\mathrm{Mg}^{2+}>\mathrm{SO}_{4}{ }^{2-}>\mathrm{K}^{+}>\mathrm{Ca}^{2+}$ \\
Lake Clifton & $\mathrm{Cl}^{-}>\mathrm{Na}^{+}>\mathrm{Mg}^{2+}>\mathrm{SO}_{4}{ }^{2-}>\mathrm{Ca}^{2+}>\mathrm{K}^{+}$ \\
Lake Preston & $\mathrm{Cl}^{-}>\mathrm{Na}^{+}>\mathrm{Mg}^{2+}>\mathrm{SO}_{4}{ }^{2-}>\mathrm{K}^{+}>\mathrm{Ca}^{2+}$ \\
Lake Pollard & $\mathrm{Cl}^{-}>\mathrm{Na}^{+}>\mathrm{Mg}^{2+}>\mathrm{SO}_{4}{ }^{2-}>\mathrm{K}^{+}>\mathrm{Ca}^{2+}$ \\
Lake Walyungup & $\mathrm{Cl}^{-}>\mathrm{Na}^{+}>\mathrm{Mg}^{2+}>\mathrm{SO}_{4}{ }^{2-}>\mathrm{K}^{+}>\mathrm{Ca}^{2+}$ \\
Shark Bay & $\mathrm{Cl}^{-}>\mathrm{Na}^{+}>\mathrm{Mg}^{2+}>\mathrm{SO}_{4}{ }^{2-}>\mathrm{Ca}^{2+} \geq \mathrm{K}^{+}$ \\
(bei zunehmender Evaporation) & $\mathrm{Cl}^{-}>\mathrm{Na}^{+}>\mathrm{Mg}^{2+}>\mathrm{SO}_{4}{ }^{2-}>\mathrm{K}^{+} \geq \mathrm{Ca}^{2+}$ \\
\hline
\end{tabular}

Tab. 7.1: Relative Ionenhäufigkeiten der Seewässer aus den fünf Salzseen und der Shark Bay.

Das nicht-konservative Verhalten des $\mathrm{Ca}^{2+}$-Ions wird durch einen Vergleich mit einem konservativen Ion $\left(\mathrm{Cl}^{-}\right)$deutlich. Die Ca-Ionen korrelieren nur im Bereich der Shark Bay mit den Cl-Ionen (Abb. 7.2). In der Shark Bay folgt das $\mathrm{Ca}^{2+}$ dem evaporativen Trend, im Lake Thetis ist es leicht, im Lake Preston und im Lake Walyungup stark erniedrigt. Dagegen ist sowohl im zentralen Lake Clifton als auch im Bereich der Grundwasserzutritte am E-Ufer das $\mathrm{Ca}^{2+}$ erhöht (max. 25,6 mmol/L). Eine Erklärung hierfür kann sein, dass $\mathrm{Ca}^{2+}$ durch die Grundwasserzutritte in den Lake Clifton eingebracht wird und dieser Eintrag die $\mathrm{Ca}^{2+}$ Verluste durch Karbonatfällungen überkompensiert.

Im Fall des Lake Thetis spricht der im Verhältnis zum Chlorid erniedrigte $\mathrm{Ca}^{2+}$-Gehalt $(11,1$ $\mathrm{mmol} / \mathrm{L}$ ) nicht gegen einen Ca-haltigen Grundwasserzutritt, da eine $\mathrm{Ca}^{2+}$-Zufuhr auch mit einem $\mathrm{Ca}^{2+}$-Entzug via Karbonatpräzipitation gekoppelt sein kann. GREY et al. (1990) beschreiben Lake Thetis als nährstoffarmen See, der durch ein sublakustrines und hochsalinares Grundwassersystem gespeist wird. Für diese These sprechen sowohl die Untersuchungen des Seewassers als auch die Untersuchungen einer Grundwasserlinse aus den Jahren 1985, 1987 und 1989 (GREY et al., 1990) im nördlich gelegenen Steinbruch, die höhere $\mathrm{Ca}^{2+}$ - und geringere $\mathrm{K}^{+}$-Konzentrationen im Grundwasserreservoir zeigten. Der Vergleich des Mg-Gehaltes mit der Alkalinität (Abb. 7.4) verdeutlicht, dass in Bezug auf den Mg-Gehalt Lake Thetis - mit Ausnahme des Seebodens in der Regenzeit - relativ angereichert und Lake Clifton relativ verarmt ist.

Die Untersuchungen an Lake Clifton belegen für die Seespiegelhochstandsphasen zusätzlich zum $\mathrm{Ca}^{2+}(11 \mathrm{mmol} / \mathrm{L})$ auch eine erhöhte Alkalinität (max. 3,5 meq/L) im Vergleich zu den Werten bei Seespiegeltiefstand (25,6 mmol/L Ca $\left.{ }^{2+} ; 2,6 \mathrm{meq} / \mathrm{L}\right)$ (Anhang 7). Die höchsten Alkalinitäten lassen sich im Bereich der Grundwasserzutritte $(6,9$ meq/L) nachweisen, woraus sich ein Mischungstrend in Richtung des Seezentrums ableiten lässt (Abb. 7.3). 
Die Werte der Alkalinität der Wasserproben aus der Regenphase vom Lake Thetis kommen ebenfalls auf dieser Linie zum Liegen, wodurch sich eventuell eine Mischung von See- und Grundwasser bestätigt.

Eine deutlich höhere Alkalinität als alle anderen NaCl-Seen zeigt Lake Walyungup, welcher mit $14 \mathrm{meq} / \mathrm{L}$ als hochalkalin bezeichnet werden kann (Abb. 7.3A).

Der pH-Wert des Lake Thetis war bei Seespiegelhochstand mit 8,25 leicht gegenüber dem $\mathrm{pH}-$ Wert bei Trockenzeit mit 8,6 erniedrigt und lag damit leicht über dem Bereich vom Standardmeerwassers $(\mathrm{pH}=8,2)$ und der Shark Bay $(8,1-8,3)$. Leicht erhöhte und geringfügig schwankende pH-Werte sind für Lake Thetis charakteristisch (GREY et al., 1990), wobei evaporitisch geprägte Randbuchten $\mathrm{zu}$ höheren $\mathrm{pH}$-Werten und leicht herabgesetzten Alkalinitäten tendieren (REITNER et al., 1996). Der pH-Wert des Lake Clifton lag in der Regenzeit knapp über 8,2 und war in der Trockenzeit mit 7,96 geringer. In der Nähe des Grundwasserzustroms lag der pH-Wert in der Trockenperiode bei 8,34. Die höchsten $\mathrm{pH}$ Werte weisen der Lake Preston-Überlauf mit 9,29, der N-Arm mit 9,1 und Lake Walyungup mit 9,14 auf (Anhang 7).

Anhand der Nährstoffgehalte lässt sich eine Einteilung in zwei Gruppen treffen. Zum einen weisen die Wässer der Shark Bay, des Lake Walyungup, des Lake Pollard und des Lake Preston sehr geringe, bzw. unterhalb des Detektionslimits liegende Gehalte an Silizium, Phosphat und Ammonium auf (Anhang 7). Zum anderen zeigen Lake Clifton und Lake Thetis besonders in Bezug auf das Silizium und das Ammonium hohe Gehalte bei ebenfalls höheren Phosphat-Gehalten. Die Ammonium-Gehalte liegen im Lake Clifton mit 37,56 $\mu \mathrm{mol} / \mathrm{L}$ in der Regenzeit und mit $60 \mu \mathrm{mol} / \mathrm{L}$ in der Trockenzeit am höchsten. In der Trockenperiode lässt sich in Lake Thetis eine Ammonium-Zunahme von ca. $1 \mu \mathrm{mol} / \mathrm{L}$ im Seewasser auf 8,8 $\mu \mathrm{mol} / \mathrm{L}$ an der Grenzschicht Sediment-Wasser und auf 70,7 $\mu \mathrm{mol} / \mathrm{L}$ im Seebodensediment beobachten. Im Sediment kommt es durch Oxidation von Schwefelverbindungen durch die SPB zur Abreicherung von Sauerstoff. Die Degradation organischer Substanzen hat durch Sulfatreduktion und Ammonifikation eine Erhöhung des $\mathrm{NH}_{4}$-Gehaltes, der Alkalinität und eine Verringerung des pH-Wertes zur Folge. Im Lake Thetis zeigen sich Schwankungen der Si-Gehalte am Seeboden während der Regenzeit mit $453 \mu \mathrm{mol} / \mathrm{L}$ und $263 \mu \mathrm{mol} / \mathrm{L}$ während der Trockenzeit. Im Lake Clifton ließ sich während der Regenzeit ein Si-Eintrag im Grundwasser-beeinflussten Bereich „Mudshore“ (943 $\mu \mathrm{mol} / \mathrm{L})$ nachweisen. In der Trockenzeit zeigt sich in der Nähe des Grundwasserzutritts kein erhöhter Si-Gehalt, während das Seewasser in dieser Zeit mit 928-942 $\mu \mathrm{mol} / \mathrm{L}$ den höchsten Si-Gehalt aufweist. 
Je nach Evaporationsgrad liegen die $\mathrm{SO}_{4}{ }^{2-}$-Gehalte knapp unter bis deutlich über den Gehalten normal marinen Wassers. Die Gehalte während der Trockenzeit von bis zu 176,5 mmol/L $\mathrm{SO}_{4}{ }^{2-}$ wurden für das Lake Preston-, 45,7 - 48,1 mmol/L für das Lake Thetis- und $57 \mathrm{mmol} / \mathrm{L}$ $\mathrm{SO}_{4}{ }^{2-}$ für das Lake Clifton-Seewasser ermittelt. Auch das Wasser der Shark Bay bei Hamelin Pool weist entsprechend hohe Gehalte auf $\left(72,7 \mathrm{mmol} / \mathrm{L} \mathrm{SO}_{4}{ }^{2-}\right)$. In der Regenperiode lagen die $\mathrm{SO}_{4}{ }^{2-}$-Gehalte generell niedriger: Lake Thetis (36,2 mmol/L), Lake Clifton (20,6 - 24,2 mmol/L), Lake Preston (max. 87,1 mmol/L) und Shark Bay (46,0-64,5 mmol/L). Für Lake Walyungup (26,6 mmol//L) und Lake Pollard (20,5 mmol/L) liegen nur Daten für jeweils eine Jahreszeit vor.

Da alle Wässer trotz teils starker evaporativer Aufkonzentration die Gipssättigung nicht erreichen, sollte ein im Vergleich zu dem sich konservativ verhaltenden Chlorid-Ion ein konstantes $\mathrm{Cl} / \mathrm{SO}_{4}{ }^{2-}$-Verhältnis ähnlich dem normal marinen Verhältnis vorliegen. Bis auf Lake Clifton und Lake Pollard zeigen viele Seen - insbesondere Lake Thetis - im Vergleich zu hohe $\mathrm{SO}_{4}{ }^{2-}-$ Gehalte auf (Abb. 7.5). Auch die Proben aus der Shark Bay liegen deutlich oberhalb der Linie des evaporativen Trends. Ältere Daten liefern für den Lake Thetis sogar noch höhere $\mathrm{SO}_{4}{ }^{2-}-$ Gehalte, die in der Regenzeit $1985109 \mathrm{meq} / \mathrm{L}$ (54,5 mmol/l) betrugen (GREY et al.,1990) und die hier vorgelegten Analysen bestätigen. Die Gehalte in Lake Walyungup lagen 1963 und 1965 bei 472-620 mg/L (COSHELL et al., 1998) (entspricht 4,90 $6,45 \mathrm{mmol} / \mathrm{L}$ ). Für die Shark Bay und Lake Clifton konnten keine Angaben zu den $\mathrm{SO}_{4}{ }^{2-}$ Gehalten in der Literatur gefunden werden.

$\mathrm{SO}_{4}{ }^{2-}$-Gehalte werden durch Sulfatreduktion zu Sulfid und $\mathrm{H}_{2} \mathrm{~S}$ oder durch Gipsfällung meist im Sediment festgelegt und sinken somit im Wasserkörper. Entsprechend müsste das Sulfat/Chlorid-Verhältnis niedriger werden. Stattdessen sind die Chlorid-Gehalte im Vergleich zu gering, insbesondere in der zum Ozean offenen Shark Bay müsste sich das Verhältnis durch die stete Nachführung und Durchmischung mit marinem Wasser angleichen. Für die Shark Bay kann eventuell von einer Eindunstung bis zur Gipsfällung ausgegangen werden, wobei Sulfat in Form von Gips im Sediment festgelegt wurde und durch Rücklösung dem System zugeführt wird. Im dem geschlossenen System des Lake Thetis muss ebenfalls eine zusätzliche Quelle für das Sulfat angenommen werden. So können z.B. salinare Grundwässer Gips aus den benachbarten Sedimenten gelöst haben. Im Lake Thetis kann das permanente „Schwefel-Recycling“ durch SPB zwar zu einer Rückführung des Sulfates aus dem Sulfid der Sedimente und Porenwässer führen, aber es kann das Sulfat/ChloridVerhältnis letztlich nur ausgleichen, nicht aber erhöhen. Trotz ähnlicher hydrochemischer Zusammensetzung der Wässer sind die SPB ausschließlich für Lake Thetis charakteristisch, 
was eventuell mit einem erhöhten Anteil an organischem Material und dadurch bedingter verstärkten $\mathrm{H}_{2} \mathrm{~S}$-Bildung via Sulfatreduktion zusammenhängen könnte.
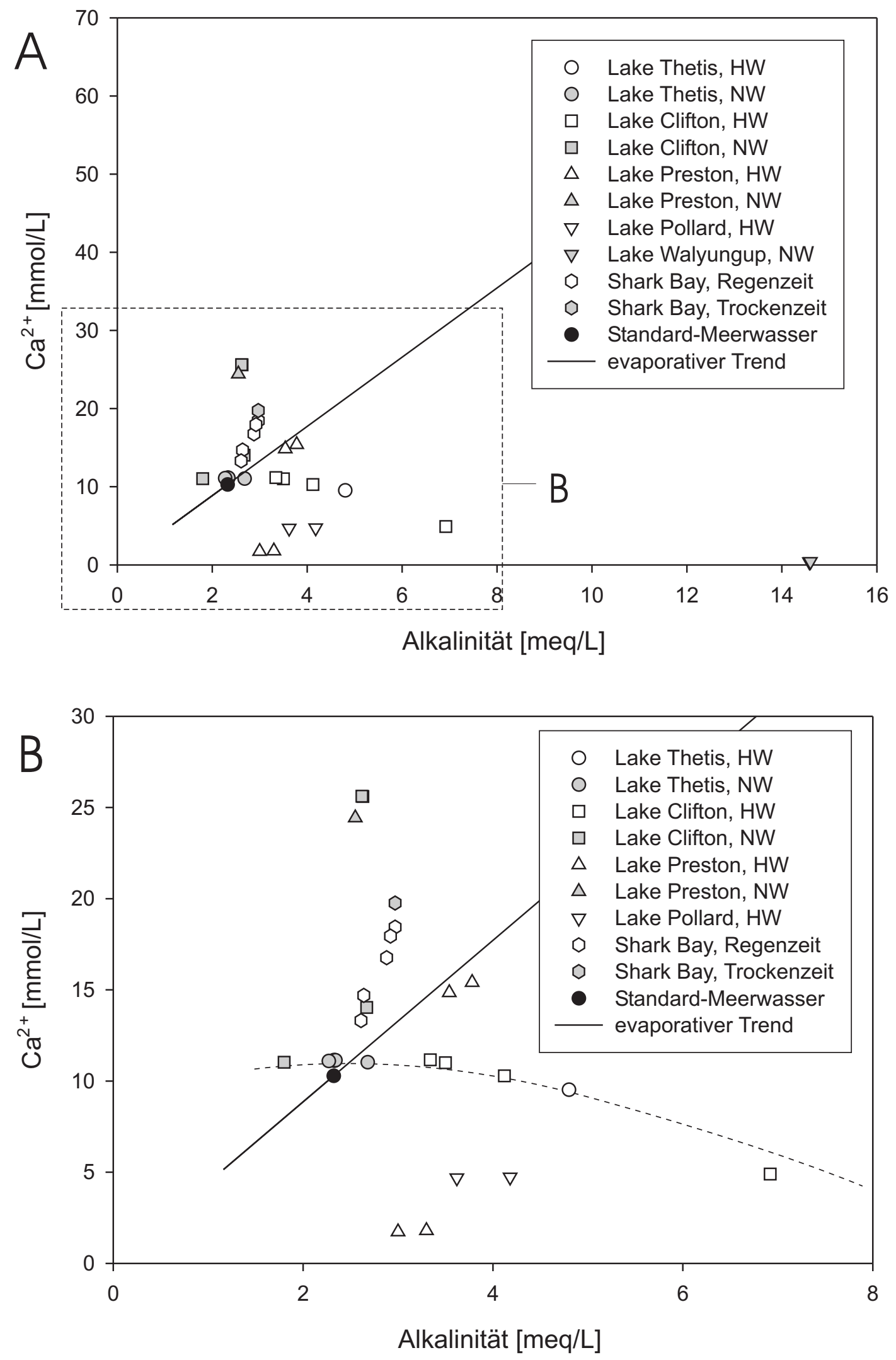

Abb. 7.3: Die $\mathrm{Ca}^{2+}$-Konzentrationen nahezu aller Seen weichen von dem evaporativen Trend ab. Lake Thetis, Lake Pollard, Lake Preston, Lake Walyungup sowie die Zuflüsse des Lake Clifton und das Seewasser in der Regenzeit enthalten zu wenig $\mathrm{Ca}^{2+}$. Die Shark Bay enthält vergleichsweise $\mathrm{zu}$ viel $\mathrm{Ca}^{2+}$. 


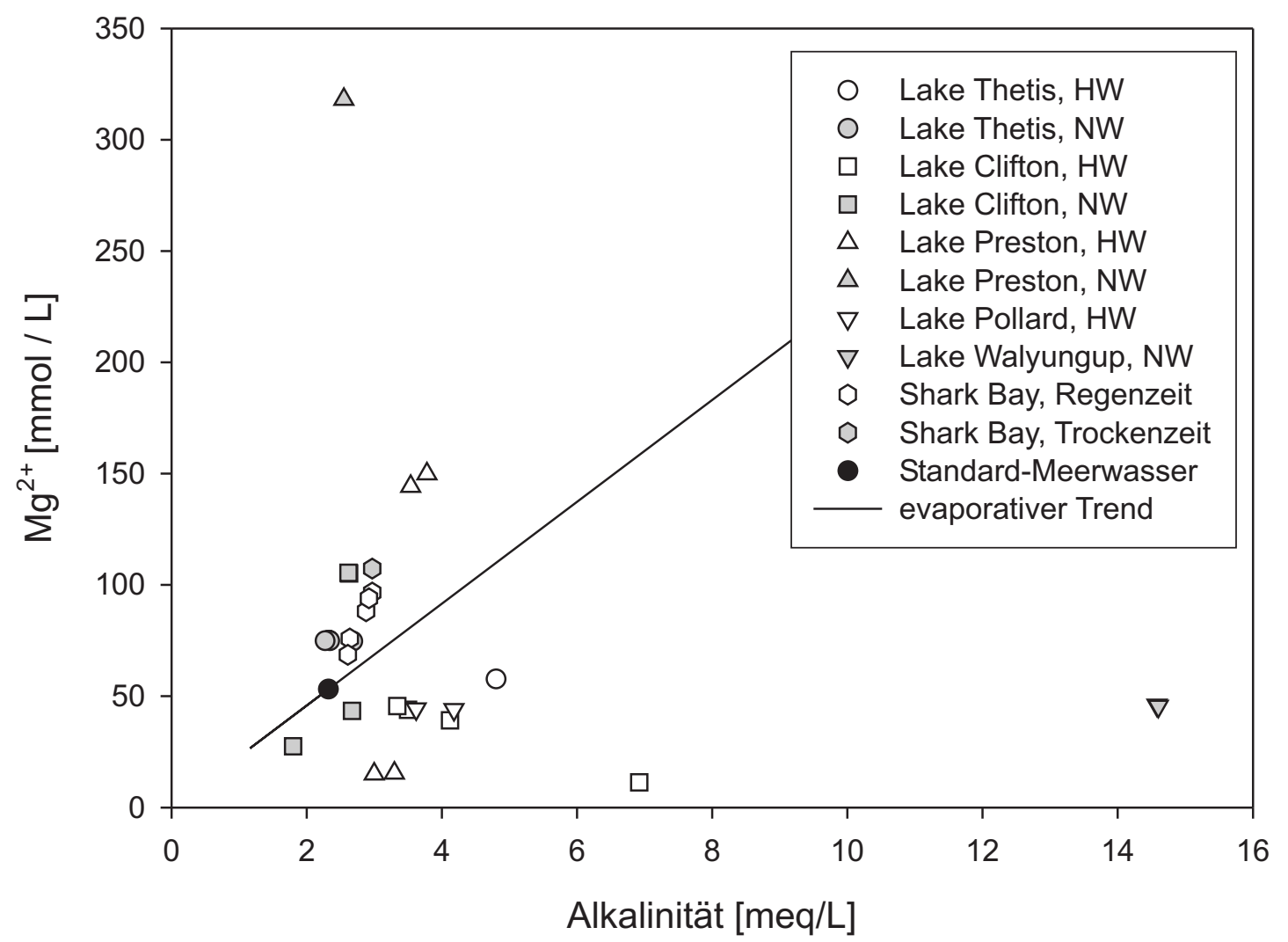

Abb. 7.4: Abweichend vom evaporativen Trend werden erhöhte, bzw. erniedrigte MgKonzentrationen deutlich.

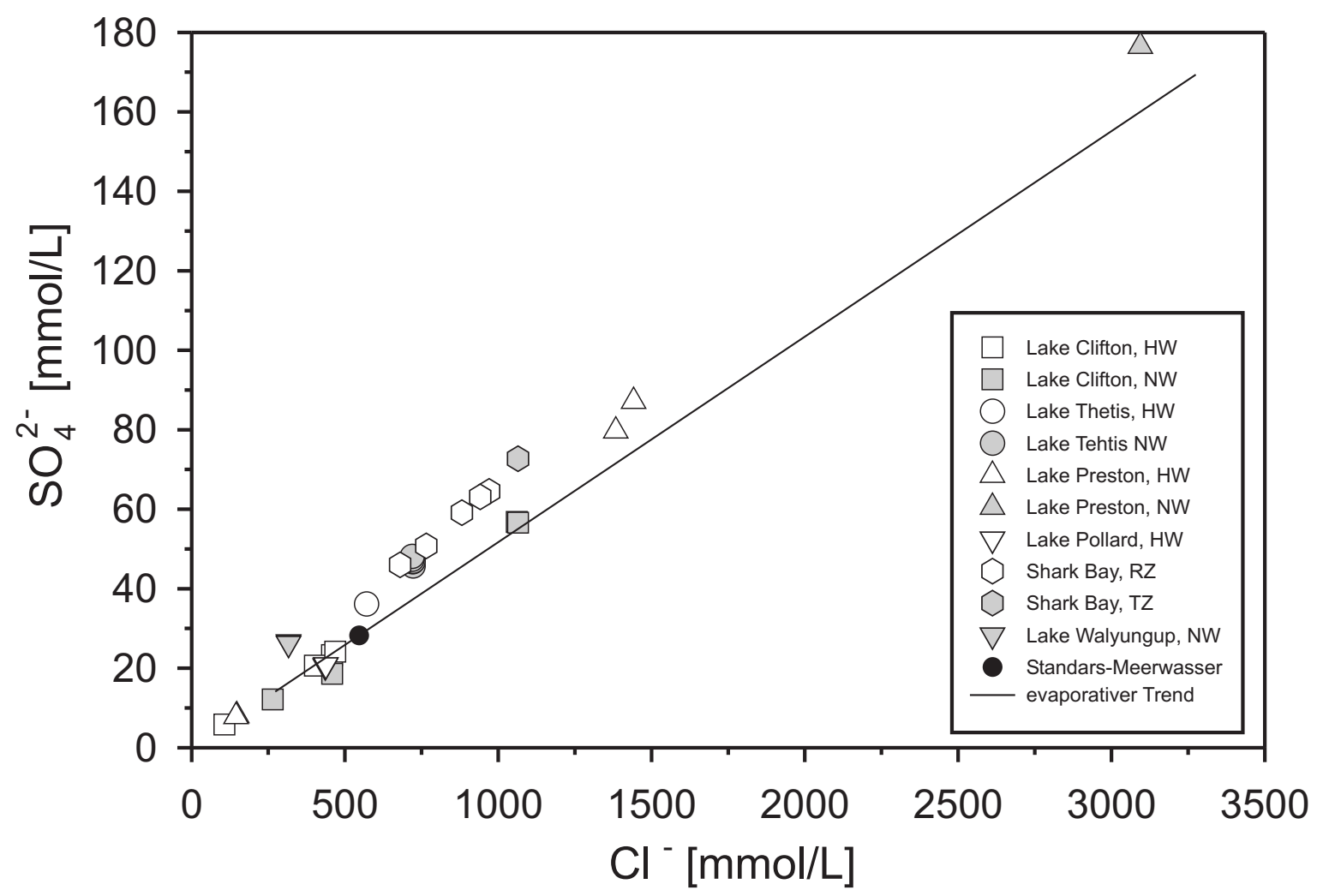

Abb. 7.5: Proben aus der Shark Bay, aus dem Lake Walyungup, Lake Preston sowie Lake Thetis weichen mit höheren Sulfat-Gehalten vom evaporativen Trend ab. 


\subsection{Sättigungszustand einzelner Mineralphasen}

Aus den hydrochemischen Daten wurden die Sättigungen bezüglich der Mineralphasen Aragonit, Kalzit und einer amorphen $\mathrm{SiO}_{2}$-Phase mit Hilfe des Computerprogramms PHREEQC (PARKHURST \& APPELlO, 1999) berechnet (Tab. 7.2). Die Fällung von Kalzit unterliegt einer kinetischen Barriere, welche erst bei einer 10fachen Übersättigung überschritten wird.

Für den Lake Thetis ergeben sich Übersättigungen bezüglich Kalzit und Aragonit von $\mathrm{SI}_{\text {Kalzit }}$ $=+0,95$ und $\mathrm{SI}_{\text {Aragonit }}=+0,81$. Das Wasser zeigt eine höhere Übersättigung als das Standardmeerwasser $\left(\mathrm{SI}_{\text {Kalzit }}=+0,76\right.$ und $\left.\mathrm{SI}_{\text {Aragonit }}=+0,61\right)$. Eine weniger starke Übersättigung zeigt die Probe aus dem obersten Seebodensediment mit $\mathrm{SI}_{\text {Kalzit }}=+0,61$ und $\mathrm{SI}_{\text {Aragonit }}=+0,46$, welche der Zone der massenhaft auftretenden SPB entspricht. In dieser Zone, die den Übergangsbereich der Sulfatreduktion/ $\mathrm{H}_{2} \mathrm{~S}-$ Oxidation darstellt, wird Sauerstoff abgereichert.

Die Übersättigungen bezüglich Aragonit und Kalzit liegen im Lake Clifton sowohl in der Regen- als auch in der Trockenzeit bei $\mathrm{SI}_{\text {Aragonit }}=0,7$ und $\mathrm{SI}_{\text {Kalzit }}=+0,8-0,9$. In der Regenzeit wird im Lake Clifton in der Mischungszone von See- und Grundwasser eine 10-fache Übersättigung in Bezug auf Kalzit (SI=1) erreicht, auf der „Mudshore“-Fläche sogar $\mathrm{SI}_{\text {Kalzit }}=$ +1,3. Der Lake Pollard weist ebenfalls eine 10-fache Kalzit-Übersättigung auf. Im Lake Preston ist die Übersättigung von Kalzit und Aragonit in der Regenzeit gegenüber der Trockenzeit leicht erhöht. Am Überlauf resultiert sogar eine 10-fache Übersättigung.

Die Seen zeigen bezüglich einer $\mathrm{SiO}_{2}$ (amorph)-Phase Untersättigungen von $-3,8$ bis $-0,1$ an, wobei für den Lake Clifton und den Lake Preston S-Arm die geringsten Untersättigungen errechnet wurden. Besonders untersättigt an $\mathrm{SiO}_{2 \text { (amorph) }}$ sind die Wässer der Shark Bay und des Lake Walyungup.

Im Vergleich zu den übrigen untersuchten Seen weist der Lake Walyungup ein starkes $\mathrm{Ca}^{2+}$ Defizit auf, wodurch ein hohes $\mathrm{Mg} / \mathrm{Ca}-$ Verhältnis von 107 und 122 resultiert (Tab. 7.3). Als Besonderheit sind in diesem See Hydromagnesit und Dolomit als primäre Fällungsprodukte vertreten (COSHELl et al., 1998). Daten von 1963 und 1965 (PASSMORE, 1970 in CoSHELl et al., 1998) weisen ein $\mathrm{Mg} / \mathrm{Ca}-$ Verhältnis von 71 in den Frühjahrsmonaten und 91 in den Herbstmonaten auf. Die Sättigungen liegen bei $\mathrm{SI}_{\text {Aragonit }}=+0,59$ bis 0,65 und bei $\mathrm{SI}_{\text {Kalzit }}=$ $+0,73$ bis $+0,79$ und sind deutlich niedriger als in den anderen untersuchten Seen.

In der Shark Bay wird bei Hamelin Pool eine jahreszeitenunabhängige, 10-fache Übersättigung in Bezug auf Kalzit erreicht. Die Aragonit-Übersättigung in der Regenzeit ist gegenüber der Trockenzeit leicht erniedrigt. Sie liegt aber dennoch mit SI Aragonit $=+0,7$ über 
der des Meerwassers $\left(\mathrm{SI}_{\text {Aragonit }}=+0,61\right)$. Im Vergleich zum Meerwasser mit einem SI Kalzit $=$ $+0,76$ sind alle Proben der Shark Bay mit SI Kalzit $=+0,9-1,0$ stärker übersättigt bei zunehmendem Trend in Bezug auf die Entfernung zum Ozeanzulauf.

\begin{tabular}{l|cccc}
\hline Westaustralische Seen \& Shark Bay & Mg/Ca & SI $_{\text {Aragonit }}$ & SI $_{\text {Kalzit }}$ & SI $_{\text {Sio2 amorph }}$ \\
\hline \hline Lake Thetis, HW & 6,1 & $+0,8$ & $+1,0$ & $-0,6$ \\
Lake Thetis, NW & $6,7-6,8$ & $+0,8$ & $+1,0$ & $-0,8--0,7$ \\
Lake Thetis Bodenwasser, NW & $6,7-6,8$ & $+0,5-+0,8$ & $+0,6-+0,9$ & $-0,8--0,7$ \\
Lake Clifton Mixing, HW & 3,8 & $+0,8$ & $+1,0$ & $-0,5$ \\
Lake Clifton Mudshore, HW & 2,3 & $+1,2$ & $+1,3$ & $-0,3$ \\
Lake Clifton, See, HW & 4,1 & $+0,7$ & $+0,9$ & $-0,5$ \\
Lake Clifton, See (nahe GW-Zufluss), NW & $2,5-3,1$ & $+0,7$ & $+0,8-+0,9$ & $-0,3--0,4$ \\
Lake Clifton, See, NW & 4,1 & $+0,7$ & $+0,8-+0,9$ & $-0,1$ \\
Lake Pollard, See, NW & $9,3-9,5$ & $+0,9$ & $+1,0$ & $-2,2$ \\
Lake Preston Überlauf, HW & 8,6 & $+0,9$ & $+1,1$ & $-1,4$ \\
Lake Preston S-Arm, HW & 9,7 & $+0,7$ & $+0,9$ & $-0,9$ \\
Lake Preston N-Arm, HW & 8,7 & $+0,8$ & $+0,9$ & $-1,3$ \\
Lake Preston S-Arm, NW & 13,0 & $+0,7$ & $+0,8$ & $-0,2$ \\
Lake Walyungup, NW & $108-122$ & $+0,6-+0,7$ & $+0,7-+0,8$ & $-2,2$ \\
SB Hamelin Pool, Regenzeit & 5,2 & $+0,8$ & $+1,0$ & $-3,0$ \\
SB L'Haridon, Regenzeit & 5,3 & $+0,7$ & $+0,9$ & $-3,0$ \\
SB Carbla Beach, Regenzeit & 5,2 & $+0,8$ & $+0,9$ & $-3,8$ \\
SB Gladstone, Regenzeit & 5,2 & $+0,7$ & $+0,9$ & $-3,6$ \\
SB Monkey Mia, Regenzeit & 5,2 & $+0,7$ & $+0,8$ & $-3,0$ \\
SB Hamelin Pool, Trockenzeit & 5,4 & $+0,9$ & $+1,0$ & $-2,0$ \\
\hline
\end{tabular}

Tab. 7.2: Mg/Ca-Verhältnisse und der Sättigungszustand bezüglich der Minerale Kalzit, Aragonit und einer amorphen $\mathrm{SiO}_{2}$-Phase in den Wässern der fünf westaustralischen Salzseen und der Shark Bay (HW: Hochwasser, NW: Niedrigwasser). 


\section{Interpretation und Diskussion}

\subsection{Rezente Mikrobialith-Bildung}

Heutige Mikrobialithe von Süßwasser-Milieus, Salz- und „Soda“-Seen werden als Modellsysteme für die Interpretation fossiler mariner und nicht-mariner Mikrobialithe in der Erdgeschichte angesehen, da unter heutigen normal-marinen Bedingungen kaum rezente Biofilmkalzifikation stattfindet. Dabei setzt das hydrochemische Makromilieu Rahmenbedingungen für kurzfristige mikrobielle Effekte. In erster Näherung können mikrobielle Stoffwechselprozesse auf das chemische Gleichgewicht des Karbonatsystems anhand von Modellrechnungen veranschaulicht und bezüglich ihrer wahrscheinlichen Auswirkungen im Mikromilieu des Biofilms diskutiert werden.

Dazu sind mehrere Annahmen nötig, um einzelne Effekte (wie z.B. C-Fixierung) in den verschiedenen Seen darzustellen. Die Temperatur ist konstant auf $20,0^{\circ} \mathrm{C}$, der Atmosphärendruck auf $p \mathrm{CO}_{2}=10^{-3,5}$ atm und die initiale Sättigung auf $\mathrm{SI}_{\text {Kalzit }}=1$ (10-fache Übersättigung) eingestellt. Für den C-Entzug durch Photosynthese wird analog zu ARP et al. (2001) eine konstante Größe von $200 \mu \mathrm{mol} / \mathrm{L}$ Kohlenstoff gerechnet. Die Differenz bezüglich der Kalzit-Übersättigung, welche sich aus der Sättigung vor und nach dem kalkulierten C-

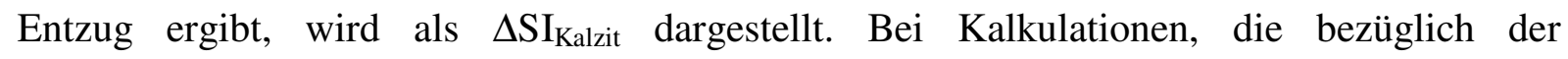
Photosynthese und Exopolymer-Degradation durchgeführt wurden, zeigte sich, dass kalzifizierte Tuben um Cyanobakterien-Filamente ( siehe z.B. RIDING, 1977; Aldabra Atoll, Seychellen) erst bei einer theoretischen Erhöhung des Sättigungsindexes für Kalzit um mehr als 0,2 Einheiten auftreten (ARP et al., 2001, 2003).

Analoge Rechnungen wurden für Lake Thetis, Lake Clifton, Lake Preston, Lake Pollard, Lake Walyungup und die Shark Bay mittels Computerprogramm PHREEQC (PARKHURST \& APPELO, 1999) durchgeführt, insbesondere, um das Ausmaß der cyanobakteriellen Beteiligung an der Karbonatfällung in den stark übersättigten Milieus abschätzen zu können (Abb. 8.1). Die Kalkulation der Sättigungen einzelner Mineralphasen ergibt ein starke Übersättigung, ohne dass spontane $\mathrm{CaCO}_{3}$-Fällungen in der Wassersäule beobachtet wurden.

Die Verkalkungen in Biofilmen sind zum einen an die Übersättigung des Umgebungsmilieus hinsichtlich karbonatischer Minerale gebunden (PENTECOST \& RIDING, 1986; RIDING, 1982). Zum anderen können in den hochhydratisierten Netzwerken aus exopolymeren Substanzen physikochemische und chemische Mikrogradienten aufgebaut und divalente Kationen aus dem Umgebungsmilieu gebunden werden. Die exopolymeren Substanzen haben die Wirkung eines Inhibitors, da die funktionellen Gruppen (Carboxyl- und Hydroxyl-Gruppen) divalente 
Kationen wie $\mathrm{Ca}^{2+}$-Ionen binden (ADDADI \& WEINER, 1989; LOWENSTAM \& WEINER, 1989; AdDADI et al., 1990; DECHO 1990, 2000ab), so dass die Fällung erst beim Überschreiten der $\mathrm{Ca}^{2+}$-Bindungskapazität und bei Zelldegradation durch freiwerdende Kationen erfolgt.

Von Karbonat eingebettete Cyanobakterien-Trichome, wie sie generell als Beleg für eine Photosynthese-induzierte Fällung angesehen werden (vgl. DUPRAZ et al., 2004), kommen sowohl im Lake Thetis als auch im Lake Clifton rezent nicht vor. Im Lake Thetis sind ausschließlich Filamentspuren der Gattung Phormidium sp. in Form unregelmäßiger ZementÜberzüge erhalten. Im Lake Clifton konnten ebenfalls von unregelmäßigem Zement überzogene Phormidium-Filamentspuren innerhalb der thrombolithischen Strukturen nachge-

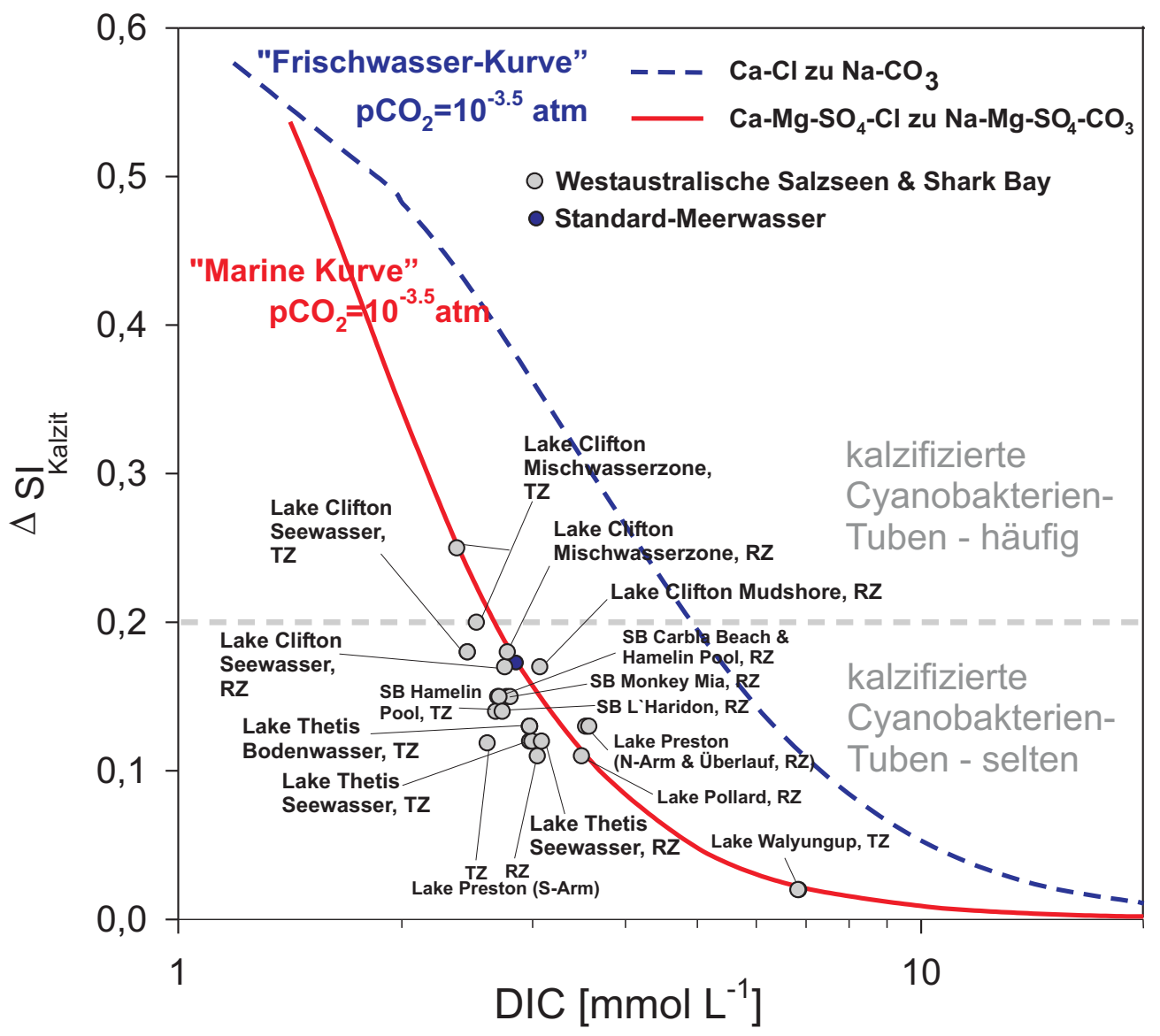

Abb. 8.1: Änderungen in der Kalzit-Übersättigung ( $\left.\Delta \mathrm{SI}_{\text {Kalzit }}\right)$ nach Einstellung der Seewässer auf eine 10-fache Übersättigung bezüglich Kalzit $\left(\mathrm{SI}_{\mathrm{Kalzit}}=1\right)$ und $p \mathrm{CO}_{2}$ $=10^{-3,5} \mathrm{~atm}$ mittels Computersimulation und anschließendem C-Entzug. Für die Shark Bay und alle Seen - mit der Ausnahme Lake Clifton - liegt der $\Delta$ SI unterhalb des Wertes für das Standardmeerwasser. Die Berechnungen für das Standardseewasser beziehen sich auf Angaben in NORDSTROM et al. (1979) (RZ: Regenzeit, TZ: Trockenzeit) (Abbildung verändert nach ARP et al., 2001). 
wiesen werden. Diese werden allerdings aufgrund der sie stabilisierenden $\mathrm{Mg}$-Si-Phase nicht als jüngste Bildungen interpretiert. Bei den Filamentspuren handelt es sich um Verkalkungszonen, welche die Filamente abbilden, ohne allerdings definierte mikritische Tubenwände aufzuweisen. Der Hauptanteil an Karbonat wird an EPS-Randbereichen von Gloeocapsa-Kolonien gebildet.

Im Fall des Lake Thetis unterstützt die Simulation des photosynthetischen C-Entzuges die histologischen Befunde. Durch die Pufferwirkung des Seewassers wird eine KalzitÜbersättigung durch eine cyanobakterielle Photosynthese nicht erhöht, der $\Delta$ SI liegt bei 0,120,13. Eine starke Pufferwirkung wirkt in „Soda“-Seen durch einen hohen DIC bei geringen $\mathrm{Ca}^{2+}$-Gehalten der Bildung Photosynthese-induzierter Cyanobakterien-Tuben entgegen; stattdessen findet eine $\mathrm{CaCO}_{3}$-Fällung erst bei sekundärer $\mathrm{Ca}^{2+}$-Freisetzung durch EPSDegradation statt (ARP et al., 1998, 2003).

In den gering alkalinen Seewässern des Lake Clifton und Lake Thetis findet eine Photosynthese-induzierte Karbonatfällung an Cyanobakterien-Trichomen, wie aus Frischwassermilieus bekannt (MERZ, 1992; MERZ-PREIß \& RIDING, 1999), nicht statt. Stattdessen liegen die Präzipitate in Lake Thetis und Lake Clifton in Form mikrokristalliner Argonitklumpen und faseriger Aragonite vor. Entsprechend liegen die Wasserproben von Lake Thetis unterhalb des Schwellenwertes von $\Delta \mathrm{SI}=0,2$. Da allerdings die Kalkulationen nur eine erste Annäherung an die natürlichen Bedingungen sein können und Filamentspuren von Phormidium sp. zementierte Umkrustungen zeigen, wird ein Photosynthese-Effekt, besonders bei herabgesetzter Diffusion innerhalb des Biofilms, nicht gänzlich ausgeschlossen. Für den Fall des Lake Clifton ist ebenfalls von einem positiven Einfluss auf die Verschiebung des Karbonatgleichgewichtes durch photosynthetische Aktivität auszugehen. In den kleinen Tümpeln (,,ponds“) auf der ebenen Fläche der erodierten Thrombolithe ist eine $\mathrm{CaCO}_{3}$ Fällung an die photosynthetische Aktivität, in diesem Fall allerdings von Grünalgen, gekoppelt. Entscheidend ist dabei eine Mischung mit meteorischen Wässern, welche die pHPufferung herabsetzten.

DEFARGE et al. (1994) beschreiben Mg-Kalzit-Fällungen an Scytonema-Filamenten in einem durch Regen-, Grund- und Meerwasser beeinflussten See (Rangiroa, Französisch Polynesien). Da neben den verkalkenden Scytonema-Filamenten unkalzifizierte Phormidium- und Microcoleus-Filamente vorkommen, wird eine verstärkte photosynthetische Aktivität von Scytonema sp. gegenüber der in den Atoll-Seen weit verbreiteten Gattung Phormidium vermutet und ein Zusammenhang zwischen Kalzifikation und Umweltbedingungen als untergeordnet bewertet (DÉFARGE et al., 1994). 
Unberücksichtigt bleiben allerdings Betrachtungen der Alkalinitätsverhältnisse und des Kohlenstoffreservoirs (DIC). Die Ergebnisse von DÉFARGE et al. (1994) lassen die Interpretation zu, dass der Eintrag meteorischer Wässer zu einer Erhöhung des $\Delta$ SI und damit zur möglichen Photosynthese-induzierten Karbonatfällung an Scytonema-Filamenten im oberflächennahen Wasser geführt hat.

Die Seen nehmen eine Position zwischen Süßwasser-Milieus auf der einen und „Soda“-Seen auf der anderen Seite ein. Eine sprunghafte pH-Wert-Erhöhung an Filamenten, wie sie für die Bildung cyanobakterieller Tuben erforderlich ist, wird durch die hydrochemische Zusammensetzung der Wässer abgepuffert. Der limitierende Faktor für die Kalzifikation der Cyanobakterien-Tuben in Lake Thetis ist dabei vermutlich weniger der DIC-Gehalt, sondern der $\mathrm{Ca}^{2+}$-Input, welcher an sublakustrine Zuflüsse gebunden ist.

In Bezug auf die Modellrechnungen für den Lake Clifton muss nochmals betont werden, dass die amorphe Mg-Si-Phase die Hauptmasse der rezenten Fällungsprodukte in den Mikrobialithen stellt. Das frühere karbonatische Mikrobialith-Wachstum steht vermutlich nicht mit den aktuellen hydrochemischen Bedingungen des Sees in Zusammenhang. Die Modellrechnungen belegen deshalb nur, dass eine Photosynthese-induzierte Fällung in Mischwasserzonen grundsätzlich möglich ist.

KONISHI et al. (2001) führen keinerlei Belege für ein rezentes Mikrobialith-Wachstum der Lake Clifton-Bioherme an, erkennen aber die Möglichkeit einer irreversiblen Schädigung des Ökosystems Lake Clifton durch stete Salinitätserhöhung. Frühere Untersuchungen bezüglich des Wasser- und Nährstoffeintrages über kultivierte Uferzonen am Lake Clifton verdeutlichen, dass die existenten Pufferzonen zu klein sind, um den Nährstoffeintrag und das Grünalgen-Wachstum (Cladophora vagabundum) zu reduzieren (DAVIES \& LANE, 1996). Durch den meso-eutrophen Status des Lake Clifton und den eutrophen bis hypereutrophen Stadien der Oberflächenzuflüsse (DAVIES \& LANE, 1996) ist der Anteil der KarbonatPräzipitate am rezenten Mikrobialith-Wachstum gering.

\subsection{Subfossile Mikrobialithe}

Die Scytonema-dominierten Biofilme in der Regenzeit und die Gloeocapsa/Entophysalisdominierten Biofilme (REITNER et al., 1996) in der Trockenzeit zeigen eine jahreszeitenabhängige Wechselfolge im Lake Thetis an. Diese findet sich in den fossilen Stromatolithen ebenfalls wieder, allerdings mit verstärkter Scytonema-Dominanz im oberen Teil. Nach GREY et al. (1990) wird durch die sedimentologischen Untersuchungen des Lake Thetis ein Übergang von marinen zu lagunären und letztlich lakustrinen Verhältnissen 
deutlich, wobei die höher gelegenen Terrassen einen höheren Seespiegel und subsequenten Rückzug des Ufers markieren. Der hohe Anteil an „Organomikrit“-Zementen im mittleren Teil der Stromatolithe belegt Phasen episodisch vadoser Verhältnisse.

Vergleichbare Regressionen spiegeln sich in den Seen Lake Clifton, Lake Preston und Lake Walyungup durch die fossilen und trockenliegenden Mikrobialithe der Ufersäume wider. Für alle Seen ist von einem klimatisch gesteuertem Seespiegelrückgang auszugehen, der auch durch Änderungen des Grundwasserreservoirs bedingt ist. Insbesondere die $\delta^{13} \mathrm{C}$ - und $\delta^{18} \mathrm{O}$ Werte in den subfossilen Mikrobialith-Proben lassen einen deutlichen evaporativen Trend in der Zeit vermissen, wie er in einem hydrologisch geschlossenen System zu erwarten wäre. Eine Mischung mit Oberflächenwasser und Wechselwirkungen mit dem Grundwasser werden dafür verantwortlich gemacht.

Die Hauptprimärproduzenten des organischen Materials der subfossilen Stromatolithe des Lake Thetis sind Cyanobakterien, Algen (Diatomeen) und Bakterien (SPB). Rezent dominieren Diatomeen und Cyanobakterien die Mikrobengemeinschaften der benthischen Biofilme, im Seebodensediment überwiegen SPB.

Die Vaccensäure ist als bakterielle Fettsäure anaerob lebender Bakterien (FANG \& BARCELONA, 1998) und aus marinen Milieus von heterotrophen Bakterien bekannt (GILAN \& SANDSTROM, 1985). Der höhere Anteil der Vaccensäure in der Gesamtgesteinsprobe gegenüber der „Organomikrit“-Probe verwundert angesichts der eingeschwemmten „flocculent mat" aus dem Seebecken. Es kann allerdings an unregelmäßigen Oberflächenstrukturen liegen, dass die eingeschwemmten SPB durch fortlaufende Karbonatfällung eingebettet werden. Eine Beteiligung der phototrophen, anoxygenen Bakterien an der Karbonatfällung wird nicht angenommen.

Die vorliegende Arbeit liefert den ersten Nachweis von Vaccensäure, welche von SPB in einem salinaren Milieu mit aktiven Mikrobialith-Wachstum gebildet wurde.

Von Cyanobakterien-Matten salinarer Seen ist bekannt, dass Biomarker der vorherrschenden Primärproduzenten Phormidium sp. und Microcoleus sp. nur geringfügig im Spektrum der extrahierbaren Lipide vertreten sind (GRIMALT et al., 1991). Aufgrund der Verteilungsmuster der Biomarker in tieferen Bereichen des Biofilms $(>3 \mathrm{~mm})$ wird postuliert, dass einige cyanobakterielle Biomarker, wie z.B. n-Heptadecan, erst nach einer Degradation der Cyanobakterien-Filamente gebildet werden (GRIMALT et al., 1991). $n$-Heptadecan ist sowohl in der subfossilen Karbonatkruste als auch in der obersten „Organomikrit“-Lage vertreten, hier allerdings nur untergeordnet. 
Von mikrobiellen Matten an heißen Quellen (Octopus Spring, Yellowstone National Park, USA (DOBSON et al. 1988; ZENG et al., 1992a; 1992b), Isländische Hot Springs (ROBINSON \& EGLiNTON, 1990), aber auch von kalzifizierten Cyanobakterien-Matten der Everglades (Schizotrix sp. und Scytonema sp.) (THIEL et al., 1997) und Scytonema-dominierten Biofilmen an Kalkstein-Fassaden (DEMBITSKY \& SREBNIK, 2002) ist $n$-Heptadecan ebenfalls bekannt. JAHNKE et al. (2004) konnten in mikrobiellen Matten und Phormidium-Kulturen aus dem Yellowstone National Park mittkettig-verzweigte Methylalkane nachweisen.

Der hohe Anteil an Diatomeen-Markern innerhalb der Stromatolithe verwundert, da Diatomeen-Schalen im Karbonat nicht enthalten sind. Während der Probenaufbereitung wurden die Schalen von der Probenoberfläche entfernt, so dass die Diatomeen-Marker aus karbonatisch eingebettetem, organischem Material stammen.

Diatomeen und Cyanobakterien lassen sich als Hauptprimärproduzenten der extrahierbaren Fettsäuren und Kohlenwasserstoffe in dem karbonatischen Material des Lake Clifton nachweisen. Die EMS-Analysen belegen allerdings einen hohen Anteil der amorphen $\mathrm{Mg}$-SiPhase in den Lake Clifton-Thrombolithen. Durch die Probenaufbereitung mittels $\mathrm{HCl}$ Aufschluss bleibt die in der Si-Phase gebundene Organik (vermutlich Diatomeen-Marker) unberücksichtigt, welche im stark hydratisierten Gerüst dieser Phase vermutet wird. Eine Aufbereitung der reinen Mg-Si-Phase mittels Flusssäure-Aufschluss wurde nicht vorgenommen, da die Phase mechanisch nicht zu trennen ist und über vorheriges Lösen des Karbonates ein nicht zuzuordnender Rest an organischem Material zurückbleiben würde.

\subsection{Bildung der amorphen Mg-Si-Phase}

Eine amorphe Mg-Si-Phase, wie sie in den Karbonaten des Lake Thetis und Lake Clifton vorkommt, ist aus dem marin-beeinflussten Satonda Crater Lake, Indonesien (ARP et al., 2003, 2004), sowie aus Salzseen wie dem alkalinen Mono Lake, Kalifornien (SouZA-EGIPSY et al., 2005), und dem Na-Cl-dominierten Great Salt Lake, Utah, USA (SPENCER et al., 1985), bekannt.

Charakteristisch für „Soda“-Seen sind allerdings Na-Silikate wie Magadiit und Kenianit (Lake Chad, N`Guigmi, Niger (SEBAG et al., 2001); Lake Magadi, Kenia (EugSTER, 1967; BEHR \& RÖHRICHT, 2000)). BEHR \& RÖHRICHT (2000) nehmen für die Silifizierung eines Silikagels über Opal-A zu Opal-C einen Prozess an, in dem biochemische Prozesse (Photosynthese-gesteuerte pH-Schwankungen) die Lösung und Fällung in Interaktion mit alkalinem Wasser und Vulkandetritus beeinflussen. 
Für Na- wie auch für Mg-Silikate bestehen Schwierigkeiten hinsichtlich ihrer Einstufung als synsedimentäre oder diagenetische Bildung und ihrer Zuordnung im fossilen Bericht durch die metastabile Kristallstruktur. Im älteren fossilen Bericht finden sich ausschließlich Cherts, da Na- und Mg-Silikate nach dem Verlust des Mg bzw. Na in reine Silikatphasen übergehen. Silifizierte Ablagerungen, wie z.B. Sinter, kommen in Heißwassermilieus vor und können als Analoge für einige präkambrische Silikat-Stromatolithe herangezogen werden (Krisuvik Hot Spring, Island (KONHAUSER et al., 2001)).

Marine karbonatische Stromatolithe des Präkambriums enthalten keine Mikrofossilien von Cyanobakterien (RIDING, 1991, 1994). Ausschließlich durch eine frühe Silifizierung der Cyanobakterien oder deren Reste sind cyanobakterielle Filamente bekannt (KNOLL \& GolubiC, 1992). Da der Silizium-Zyklus rezenter Salzseen an die Lösung vulkanischer Silikatgläser (KEMPE, 1990) und im Fall der Seen Lake Thetis und Lake Clifton an die biogene Silikatabscheidung, die Lösung von Diatomeen-Schalen und einer Repräzipitation gekoppelt ist (GREY et al., 1990), ist ein direkter Vergleich zu präkambrischen SilikatPräzipitaten, die auf anorganische Reaktionen (SIEVER, 1992) und die Dominanz vulkanoklastischer Sedimente zurückgehen, nicht möglich.

Vom Mono Lake gibt es Hinweise auf eine EPS-induzierte Silifizierung (SoUZA-EGIPSY et al., 2005). Vom Satonda Crater Lake (Meerwasser-Äquivalent mit erhöhter Alkalinität) ist ebenfalls eine amorphe Mg-Si-Phase beschrieben (ARP et al., 2003; 2004). Vom Great Salt Lake ist eine authigene $\mathrm{Mg}$-Si-Phase bekannt, die ein $\mathrm{Mg} / \mathrm{Si}$-Verhältnis von 1:1 aufweist. Vermutlich wurde die Phase nach Lösung der Diatomeen-Schalen im oberen Sediment gebildet (SPENCER et al., 1985). Die Mg-Si-Phase des Satonda Crater Lake weist ebenfalls ein Mg/Si-Molverhältnis von 1:1 auf (ARP et al., 2003). Die Mg-Si-Präzipitate vom Mono Lake haben ein $\mathrm{Mg} / \mathrm{Si}$-Verhältnis von ca. 1 : 1,66 (SOUZA-EGIPSY et al., 2005), welches dem der Mg-Si-Phase des Lake Clifton entspricht und dem der Phase vom Lake Thetis mit 1:1,25 bis $1: 1,43$ nahe kommt.

An das Vorhandensein von Mikroorganismen ist die Fällung von amorphen Spherulithen (,silica spheroids“, RENAUT et al., 1998) in hydrothermalen Wässern gebunden, welche epizellulär und/oder intrazellulär zytoplasmische Substanzen ersetzten (KONHAUSER \& FERRIS, 1996; RENAUT et al., 1998; WEE et al., 2001).

Die gut erhaltenen silifizierten Scytonema-Scheiden der subfossilen Stromatolithe weisen auf eine rasche Imprägnation hin. In situ-Mineralisierungen von Mikroorganismen und Biofilmen, wie z.B. in dem durch heiße Quellen gespeisten Lake Bogoria (Kenia) können noch zu Lebzeiten der Mikroben einsetzen und Mikrostromatolithe aufbauen (RENAUT et al., 1998). 
Die Affinität der Kieselsäure, sich an funktionelle Gruppen innerhalb der Biofilm-EPS zu binden, liefert die Vorraussetzung für eine rasche Silifizierung (RENAUT et al., 1998).

Durch die EPS werden divalente Kationen an den sauren Molekül-Gruppen komplexiert, so dass für die Fällung der Kieselsäure - wie auch bei der Karbonatpräzipitation - die Bindungskapazität überschritten sein muss und die Kationen (z.B. $\mathrm{Ca}^{2+}$ und $\mathrm{Mg}^{2+}$ ) freigesetzt werden. Dieser Prozess setzt vermutlich erst bei Zersetzung des organischen Materials ein.

Für die Cyanobakterien-Scheiden wird eine frühe Silifizierung durch die amorphe $\mathrm{Mg}-\mathrm{Si}$ Phase favorisiert, bei der die Polysaccharide über „cross links“ die Struktur der Scheiden beibehalten (DECHO, 1990). Dieser Vorgang kann sowohl rasch bei noch nicht vollständigem Zellzerfall als auch in strukturlosen Zerfallsprodukten erfolgen. SOUZA-EGIPSY et al. (2005) konnten dagegen eine Zunahme im Silizium-Anteil von absterbenden Zellwänden zu fossilen Zellwänden beobachten. Die Mg-Si-Präzipitation ist an die Oberflächen der - hier allerdings endolithischen - Cyanobakterien und EPS gebunden und verläuft subsequent während der Zersetzung des Biofilms („Post-mortem events“) (SouZA-EGIPSY et al., 2005).

Einer Remineralisation von vormals karbonatisch erhaltenen Scheiden widersprechen die fehlenden Lösungserscheinungen. Auch sind die Phasenübergänge zu den „Organomikrit“Lagen scharf, in Richtung der Aragonite dagegen häufig diffus. Die Schrumpfungsrisse der stark hydrolysierten Mg-Si-Phase gehen nicht in die Karbonat-Phase über. Stellenweise sind allerdings Risse und Klüfte innerhalb der Karbonate mit der amorphen Mg-Si-Phase gefüllt, so dass zum Teil auch von einer sekundären Bildung ausgegangen werden kann. Eine Fällung erfolgte vermutlich innerhalb leicht lithifizierter Karbonate. Zudem wird die z.T. nicht lagig entwickelte $\mathrm{Mg}$-Si-Phase als allochtoner Eintrag der im oder am Seeboden präzipitierten $\mathrm{Mg}$ Si-Phase, wie sie von GREY et al. (1990) nachgewiesen wurde, interpretiert.

Während der Präzipitation der Aragonite müssen Phasen erhöhter Lösung von DiatomeenSchalen und Repräzipitation existiert haben, die durch geänderte Umweltbedingungen (Eutrophierung, pH-Schwankungen, Salinitätsänderungen), oder/und durch biologischinduzierte Mikrogradienten im Bereich der Biofilme hervorgerufen wurden. Der pH-Wert des Lake Thetis beträgt 8,2-8,6, so dass für die Fällung entweder von einer $\mathrm{pH}$-Änderung des Seewassers oder des Mikromilieus ausgegangen werden muss. Zur Fällung von MgHydrosilikaten sind die entscheidenden Kriterien der $\mathrm{pH}-$ Wert, die Salz- und $\mathrm{Mg}^{2+}$ Konzentration sowie die Temperatur (KENT \& KASTNER, 1985). Die $\mathrm{Mg}^{2+}$-Adsorption und damit zusammenhängende $\mathrm{Mg}$-Si-Präzipitation (hier Hydrosilikat-Präzipitation) nimmt bei zunehmender NaCl-Konzentration ab. Eine Adsortion findet nur bei einer $\mathrm{NaCl}$ Konzentration von < 0,05 M statt (KENT \& KASTNER, 1985). 
Im Lake Clifton liegt zur Zeit der Probennahme der Hauptanteil an rezenten Mineralbildungen auf Seiten der amorphen $\mathrm{Mg}$-Si-Phase, die als primäre Phase angesehen wird. Die Mg-Si-Phase wird in der Arbeit von MoORE (1993) sowie in weiteren Arbeiten über den Lake Clifton (MoORE et al., 1983; MoOre, 1987; MoORE \& BurNE, 1994) nicht erwähnt, so dass davon auszugehen ist, dass sich die Phase vermutlich erst nach 1994 gebildet hat und mit der Eutrophierung des Sees zusammenhängt. Zum damaligen Zeitpunkt war der See noch intakt, aktives Thrombolith-Wachstum war an das Auftreten von Scytonema sp. gekoppelt. Heute befindet sich der See in einem Zustand zunehmender Eutrophierung. Die früheren Scytonema-dominierten Biofilme sind nicht mehr dominierend. Stattdessen sind Diatomeen und Grünalgen die vorherrschenden Primärproduzenten.

Zeitlich lässt sich der Zeitraum der Mg-Si-Bildungen aufgrund der ${ }^{14} \mathrm{C}$-Datierungen in GREY et al. (1990) einschränken. Die tieferen Teile der Stromatolithe haben Alter zwischen 1210 und 1080 Jahren, die äußere Kruste (äquivalent mit Stromatolith-Typ 3 \& 4) weist ein Alter von 90 - 5 Jahren auf. Setzt man eine synsedimentäre Bildung der Mg-Si-Phase voraus, so können jüngste Bildungen ein maximales Alter von 15 Jahren haben. Sedimentkerne, welche zwischen 1980 und 1989 genommen wurden, enthalten in rezenten Ablagerungen (,present lake deposits“, GREY et al., 1990) eine amorphe Mg-Si-Phase, so dass davon ausgegangen wird, dass zu dieser Zeit die Mg-Si-Phase noch rezent gebildet wurde. In den rezenten Biofilm-Proben kommt keine Mg-Si-Phase vor. Demnach wird eine Änderung der Umweltbedingungen nach 1990 im Lake Thetis angenommen, welche durch einen Rückgang der Eutrophierung und Änderungen des Grundwasserreservoirs (eventuell mit einer Verbindung zum Indischen Ozean) hervorgerufen sein könnte.

Aufgrund der amorphen Struktur wird die Mg-Si-Phase vielfach übersehen. GREY et al. (1990) bemerken zwar eine amorphe Phase, allerdings nur in den Beckensedimenten und nicht in den subfossilen Stromatolithen. So erkennen KEMPE \& KAZMIERCZAK (1993) zwar silifizierte Cyanobakterien-Lagen im Satonda Crater Lake, interpretieren eine amorphe MgSi-Phase (ARP et al., 2003, 2004) allerdings als primären Mg-Kalzit (vgl. KAZMIERCZAK \& KEMPE, 2004).

Die amorphen $\mathrm{Mg}$-Si-Mineralphasen, welche bis dato als Randerscheinungen eingestuft wurden, können in Diatomeen-reichen Salzseen, in denen $\mathrm{Mg}$ in ausreichender Menge bereitgestellt wird, als charakteristisches Fällungsprodukt interpretiert werden. Zum besseren Verständnis der Bildungsprozesse (z.B. Zusammenhang pH, Salinitäten, Diffusionsgradienten im Biofilm, Eutrophierung) und synsedimentärer oder diagenetischer Abfolgen sind weitere Untersuchungen an bekannten Fallbeispielen, wie auch äquivalenten, salinaren Milieus nötig. 


\subsection{Fossiles Vergleichsbeispiel}

Als fossiles Vergleichsbeispiel für den Lake Thetis kann der miozäne Ries-Krater-See (Süddeutschland) herangezogen werden. Ein Teil der Riessee-Karbonate zeigt deutliche Übereinstimmungen mit den Stromatolithen des Lake Thetis. Die laminaren „Sinterkrusten“ der Cladophorites-Bioherme des Riessees ähneln denen der in den Lake ThetisStromatolithen ausgebildeten „Organomikrit“-Krusten. Zudem sind ausschließlich in jungen Ries-See-Ablagerungen Cyanobakterien gefunden worden, die morphologisch der Scytonema sp. des Lake Thetis ähnlich sind („,?Cyanobakterien-Typ 62“: vgl. S. 128; Tafel 19, Fig. 2-4 in ARP (1994); Tafel 16, Fig. 8, in ARP (1995)). Entsprechend kann ein Teilstadium des RiesKrater-Sees, wie es im oberen Teil des Profils „Hainsfarth“ aufgeschlossen ist, als fossiles Beispiel für den Lake Thetis angesehen werden.

Zwar sind ältere Bereiche des „Soda“-See-Stadiums mit den typischen Spring-Mounds, wie sie an sublakustrinen Quellen im Ries-See gebildet werden (ARP, 1995; PACHE et al., 2001), nicht mit Lake Thetis vergleichbar. Allerdings wird für den oberen Teil der im Profil „Hainsfarth“ aufgeschlossenen Bioherme eine Entstehung in einem See mit einer Hydrochemie angenommen, wie sie dem des Lake Thetis ähnlich ist. Im alkalinen RiesKrater-See fand ein Wechsel vom „Soda“-See über Playa-Stadien hin zu einem Aussüßungsstadium statt (JANKOWSKI, 1981; ARP, 1995), der den generellen Trend zu mehr humidem Klima zum Ende des Miozäns widerspiegelt.

Allerdings wird für den Ries-See eine primäre Mg-Kalzit-Fällung diskutiert. Entsprechende Fällungsprodukte wurden im Zuge einer phreatischen Mischwasser-Dolomitisierung größtenteils umkristallisiert (ARP, 1995). Eine primär aragonitische Mineralogie charakterisiert die „Spring Mounds“ (ARP, 1995; PACHE et al., 2001), die aus dem „Soda“See-Stadium stammen. 


\section{Resümee}

- Erstmalig konnte eine amorphe Mg-Si-Phase in den subfossilen Stromatolithen des Lake Thetis und als rezente Mineralphase in den Lake Clifton-Thrombolithen nachgewiesen werden. In den Stromatolithen des Lake Thetis führte eine rasche Imprägnierung zu gut erhaltenen, schwach silifizierten CyanobakterienMikrofossilien.

- Das $\mathrm{Mg} / \mathrm{Si}$-Verhältnis ist dem Verhältnis anderer $\mathrm{Mg}$-Si-Phasen in mikrobiellen Karbonatbildungen in Salz- und „Soda“-Seen ähnlich. Die amorphe Mg-Si-Phase wird als charakteristisches Fällungsprodukt in Salzseen interpretiert, in denen Silizium und Magnesium in ausreichender Menge bereitgestellt werden.

- Die Biofilme des Lake Thetis werden während der Regenzeit von Scytonema sp. und Phormidium sp. dominiert, während der Trockenperiode haben kokkale Cyanobakterien wie Gloeocapsa sp. / Entophysalis sp. ihre Hauptwachstumsphase. Im Sublitoral des Lake Thetis ist ein rezentes Stromatolith-Wachstum während der Trockenzeit in EPS-Randbereichen kokkaler Cyanobakterien-Kolonien (Gloeocapsa sp.) und in Form Phormidium-Filament-Steinkerne an kranzartigen Säumen der Bioherm-Basis festzustellen. Während der Regenzeit findet eine Karbonatpräzipitation in der Umgebung von Scytonema-Filamenten statt.

- Die hydrochemischen Modellrechnungen belegen, dass ein photosynthetischer CEntzug in Mischwasserzonen des Lake Clifton $\mathrm{zu}$ einer Verschiebung des Karbonatgleichgewichtes führen kann. Im Lake Thetis wird eine photosynthetisch bedingte Verschiebung des Gleichgewichtes aufgrund der verkalkten EPSRandbereiche und zementierten Filamentspuren nicht ausgeschlossen. Mikritische Cyanobakterien-Tuben werden dennoch rezent in beiden Seen nicht gebildet.

- Biomarker-Analysen einer stromatolithischen Karbonatkruste vom Lake Thetis belegen den Eintrag hochdiverser Biomasse aus Cyanobakterien, aeroben und anaeroben Bakterien und Diatomeen, und zeigen an, dass sich die subfossile Mikrobengemeinschaft nicht von der Rezenten unterscheidet. Trotz fehlendem Einfluss der Schwefelpurpurbakterien auf die Karbonatfällung an der StromatolithOberfläche stammt das Biomarker-Hauptsignal innerhalb der subfossilen Stromatolithe von den Schwefelpurpurbakterien. 
- Ein Biomarker für Schwefelpurpurbakterien (Vaccensäure) konnte erstmalig in einem lakustrinen, durch aktives Mikrobialith-Wachstum geprägten Milieu und in den darin gebildeten, subfossilen Stromatolithen nachgewiesen werden.

- Die Mikrobialithe des Lake Thetis zeigen keinen eindeutigen evaporativen Trend. Die Cyanobakterien-Lamellen weisen eine negative Kovarianz auf, welche zum einen dem Einfluss von Überschichtungen im Flachstwasser mit meteorischem Wasser und damit zusammenhängende geringere pH-Pufferung, Photosynthese-Effekte sowie der Primärproduktion und zum anderen dem Einfluss evaporitisch geprägtem Seewasser und isotopisch schwererem Grundwasser zugeschrieben wird. Übergeordnet ergibt sich ein leichter Trend in der Zeit von gleichbleibenden bis schwerer werdenden hin zu leichteren $\delta^{18} \mathrm{O}$ - und $\delta^{13} \mathrm{C}-\mathrm{Werten}$. 


\section{Literaturverzeichnis}

Addadi, L. \& Weiner, S. (1985): Interactions between acidic proteins and crystals: stereochemical requirements in biomineralization.- Proceedings of the National Academy of Science USA, 82: 4110-4114.

Awramik, S.M \& Vanyo, J.P (1986): Heliotrophism in modern stromatolites.- Science, 231: 1279-1281.

Embry, A.F. \& Klovan, E.J. (1972): Absolute water depth limits of Late Devonian paleoecological zones.- Geologische Rundschau, 61: 672-686.

Addadi, L. \& Weiner, S. (1989): Stereochemical and structural relations between macromolecules and crystals on biomineralization.- [In]: Mann, S., Webb, J. \& Williams, R.J.P (Eds.): Biomineralization: 133-156, Weinheim (VCH Verlagsgesellschaft).

Addadi, L., Berman, A., Moradian-Oldak, J. \& Weiner, S. (1990): Tuning of crystal nucleation and growth by proteins: molecular interactions at solid-liquid interfaces in biomineralization.- Croatica Chemica Acta, 63: 539-544.

Aitken, J. D. (1967): Classification and environmental significance of cryptalgal limestones and dolomites, with illustrations from the Cambrian and Ordovician of southwestern Alberta. Journal of Sedimentary Petrology, 37: 1163-1178.

Arp, G. (1994): Algenflore und Fazies der miozänen Karbonate am Nordrand des Kratersees im Nördlinger Ries (Süddeutschland).- Unveröffentlichtes Manuskript, Diplomarbeit, Friedrich Alexander Universität Erlangen-Nürnberg.

Arp, G. (1995): Lacustrine bioherms, spring mounds, and marginal carbonates of the Riesimpact-crater (Miocene, Southern Germany).- Facies, 33: 35-90.

Arp, G., Hofmann, J. \& Reitner, J (1998): Microbial fabric formation in spring mounds ('Microbialites') of alkaline salt lakes in the Baran Jaran Sand Sea, PR China.- Palaios, 131: 581-592.

Arp, G., Thiel, V., Reimer, A., Michaelis, W. \& Reitner, J. (1999a): Biofilm exopolymers control microbialite formation at thermal springs discharging into the alkaline Pyramid Lake, Nevada, USA.- Sedimentary Geology, 126: 159-176.

Arp, G., Reimer, A. \& Reitner, J. (1999b): Calcification in cyanobacterial biofilms of alkaline salt lakes.- European Journal of Phycology, 34: 393-403.

Arp, G., Reimer, A. \& Reitner, J. (2001): Photosynthesis-induced biofilm calcification and calcium concentrations in Phanerozoic oceans.- Science, 292, 1701-1704.

Arp, G., Reimer, A. \& Reitner, J. (2003): Microbialite formation in seawater of increased alkalinity, Satonda Crater Lake, Indonesia. Journal of Sedimentary Research 73: 105-127.

Arp, G., Reimer, A. \& Reitner, J. (2004): Microbialite formation in seawater of increased alkalinity, Satonda Crater Lake, Indonesia - Reply. -Journal of Sedimentary Research, 74: 318-325.

Awramik, S.M. (1971): Precambrian columnar stromatolite diversity: reflection of metazoan appearance. Science, 174: 825-827.

Bauld, J. (1986): Benthic microbial communities of Australian saline lakes. [In:] De Dekker, P. \& Williams, W.D. (Eds.): Limnology in Australia: 95-111.

Behr, H.-J. \& Röhricht, C. (2000): Record of seismotectonic events in siliceous cyanobacterial sediments (Magadi cherts), Lake Magadi, Kenya.- International Journal of Earth Sciences, 89: 268-283. 
Berner, R.A. (1998): The carbon cycle and CO2 over Phanerozoic time: the role of land plant.Philosophical Transactions of the Royal Society of London, B, 353: 75-82.

Bobbie, R.J. \& White, D.C. (1980): Characterization of benthic microbial community structure by High-Resolution Gas Chromatography of fatty acid methyl esters.- Applied and Environmental Microbiology, 39, 6: 1212-1222.

Böhm, F. \& Brachert, T.C. (1993): Deep-water stromatolites and Frutexites MASLOV from the Early and Middle Jurassic of S-Germany and Austria.- Facies, 28: 145-168.

Boon, J.J., De Leeuw, J.W., v. d. Hoek, G.J., Vosjan, J.H. (1977): Significance and taxonomic value of iso and anteiso monoenoic fatty acids and branched $\beta$-hydroxy acids in Desulfovibrio desulfuricans.- Journal of Bacteriology, 129: 1183-1191.

Boskey, A.L. (1996): Matrix proteins and mineralization : an overview.- Connective Tissue Research, 35: 357-363.

Braithwaite, C.J.R. \& Zedef, V. (1996): Hydromagnesite stromatolites and sediments in an alkaline lake, Salda Gölü, Turkey.- Journal of Sedimentary Research, 66: 991-1002.

Buick, R. \& Groves, D.I. (1995): Abiological origin of described stromatolites older than 3,2 Ga: Comment and reply.- Geology, 23: 191-192.

Burne, R. V. \& Moore, L. S. (1993): Microatoll microbialites of Lake Clifton, Western Australia: morphological analogues of Crytozoön proliferum HALL, the first formallynamed stromatolite.- Facies, 29: 149-168.

Burne, R.V. \& Moore, L.S. (1987): Microbialites: organosedimentary depositis of benthic microbial communities.- Palaois, 2: 241-254.

Canfield, D.E. (1998): A new model for Proterozoic ocean chemistry.- Nature, 396: 450-453.

Coshell, L., Rosen, M.R. \& McNamara, K.J. (1998): Hydromagnesite replacement of biomineralized aragonite in a new location of Holocene stromatolites, Lake Walyungup, Western Australia.- Sedimentology, 45: 1005-1018.

Costerton, J.W., Lewandowski, Z., Caldwell, D.E., Korber, D.R. \& Lappin-Scott, H.M. (1995): Microbial biofilms.- Annual Review in Microbiology, 49: 711-746.

Davies, P.M. \& Lane, J.A.K. (1996): The impact of vegetated buffer zones on water and nutrient flow into Lake Clifton, Western Australia.- Journal of the Royal Society of Western Australia, 79: 155-160.

Decho, A.W. (1990): Microbial exopolymer secretions in ocean environments: Their role(s) in food webs and marine processes.- Oceanography and Marine Biology Annual Review, 28: 73-153.

Decho, A.W. (2000a): Exopolymer microdomains as a structuring agent for heterogeneity within microbial biofilms.- [In]: Riding, R. \& Awramik, A.M. (Eds.): Microbial Sediments: 9-15, Springer, Berlin.

Decho, A.W. (2000b): Microbial biofilms in Intertidal systems: an overview.- Continental Shelf Research, 20: 1257-1273.

Défarge, C., Trichet, J. \& Couté, A. (1994): On the appearance of cyanobacterial calcification in modern stromatolites.- Sedimentary Geology, 94: 11-19.

Défarge, C. \& Trichet, J. (1995): From biominerals to „organominerals“: The example of the modern lacustrine calcareous stromatolites from Polynesian atolls.- Bulletin de 1'Institut océanographique Monaco, no. spec., 14: 265-271. 
Dembitsky, V.M. \& Srebnik, M. (2002): Variability of hydrocarbon and fatty acid components in cultures of the filamentous cyanobacterium Scytonema sp. isolated from microbial community "Black Cover" of limestone walls in Jerusalem.- Biochemistry, 67: 12761282.

Dobson, G., Ward, D.M., Robinson, N. \& Eglinton, G. (1988): Biogeochemistry of hot spring environments: extractable lipids of a cyanobacterial mat.- Chemical Geology, 68: 155.179.

Dunham, R.J. (1962): Classification of carbonate rocks according to depositional texture.- [In:] Ham, E.W. (ed.): Classification of carbonate rocks.- American Association of Petroleum Geologists, Memoir, 1: 108-121.

Dupraz, C., Visscher, P.T., Baumgartner, L.K. \& Reid, R.P. (2004): Microbe-mineral interactions: early carbonate precipitation in a hypersaline lake (Eleuthera Island, Bahamas).- Sedimentology, 51, 4: 745-765.

Edlund, A., Nichols, P.D., Roffey, R. \& White, D.C. (1985): Extractable and lipopolysaccharide fatty acid and hydroxy acid profiles from Desulfovibrio species.Journal of Lipid Research, 26: 982-988.

Ekart, D.D, Cerling, E.T., Montanez, I.P. \& Tabor, N.J. (1999): 400 million year carbon isotope record of pedogenic carbonate; implications for paleoatmospheric carbon dioxide.- American Journal of Science, 299, 10: 805-827.

Eugster, H.P. (1967): Hydrous sodium silicates from Lake Magadi, Kenya; precursors of bedded chert.- Science, 157: 1177-1180.

Eugster, H.P. \& Hardie, L.A. (1978): Saline Lakes.- [In:] Lerman, A. (Ed.): Lakes. Chemistry. Geology. Physics: 237-293, Springer, New York.

Fang, J. \& Barcelona, M.J. (1998): Biogeochemical evidence for microbial community change in a jet fuel hydrocarbons-contaminated aquifer.- Organic Geochemistry, 29: 899-907.

Flügel, E. (1982a): Recent carbonate sedimentation.-[In:] Flügel, E.: Microfacies analysis of Limestones: 27-61, Springer, Berlin.

Flügel, E. (1982b): Microfacies characteristics.-[In:] Flügel, E.: Microfacies analysis of Limestones: 107-263, Springer, Berlin.

Flügel, E. (2004): Microfacies of Carbonate Limestone Rocks. Analysis, Interpretation and Application. 976 S. Springer-Verlag.

Folk, R.L. (1959): Practical petrographical classification of limestones.- American Association of Petroleum Geologists, Bulletin, 68: 1-38.

Garcia-Pichel, F., Al-Horani, F.A., Farmer, J., Ludwig, R. \& Wade, B.D. (2004): Balance between microbial calcification and metazoan bioerosion in modern stromatolitic oncolites.- Geobiology, 2: 49-57.

Gelpi, W., Schneider, H., Mann, J. \& Oró., J. (1970): Hydrocarbons of geochemical significance in microscopic algae. Phytochemistry, 9: 603-012.

Gillan, F.T. \& Sandstrom, M.W. (1985): Microbial lipids from a nearshore sediment from Bowling Green Bay, North Queensland: The fatty acid composition of intact lipid fractions.- Organic Geochemistry, 8: 321-328.

Grasshoff, K., Ehrhardt, M. \& Kremling, K. (Eds.) (1983): Methods of seawater analysis: Second, revised and extended edition. 419S., Verlag Chemie, Weinheim. 
Grey, K., Moore, L.S., Burne, R.V., Pierson, B.K. \& Bauld, J. (1990): Lake Thetis, Western Australia: an example of saline lake sedimentation dominated by benthic microbial processes.- Australian Journal of Marine Freshwater Research, 41: 275-300.

Grimalt, J.O., De Witt, R., Teixidor, P. \& Albaigés, J. (1991): Lipid biogeochemistry of Phormidium and Microcoleus mats.- Organic Geochemistry, 19: 50-530.

Grotzinger, J.P. \& Rothmann, D.H. (1996): An abiotic model for stromatolite morphogenesis.Nature, 383: 423-425.

Grotzinger, J.P. (1990): Geochemical model for Proterozoic stromatolite decline. - American Journal of Science, 290-A: 80-103.

Grotzinger, J.P. (1994): Trends in Precambrain carbonate sediments and their implication for understanding evolution.- [In:] Bengtson, S. (Ed.): Early Life on Earth.- Nobel Symposium, 84: 245-258, Columbia University Press, New York.

Hardie, L.A. (1996): Secular variantions in seawater chemistry: an explanation for the coupled secular variation in the mineralogies of marine limestones and potash evaporates over the past 600 m.y.- Geology, 24: 279-283.

Hayes, J.M. (1994): Global methanotrophy at the Archaen-Proterozoic transition.- [In:] Bengtson, S. (Ed.): Early life on Earth.- Nobel Symposium, 84: 220-236, Columbia University Press, New York.

Hefter, J. (1997): Biogeochemie löslicher und makromolekular gebundener organischer Verbindungen in Sedimenten, Erdölen und Kontaminierten Böden. - Dissertation, Universität Hamburg.

Holland, H.D. (1992): Chemistry and evolution of the Proterozoic ocean.- [In:] Schopf, J.W. \& Klein, C. (Eds.): The Proterozoic Biosphere: 169-172, University Press, Cambridge.

House, C.H., Schopf, J.W., McKeegan, K. D., Coath, C. D., Harrison, T.M. \& Stetter, K.O. (2000): carbon isotopic composition of individual Precambrian microfossils. - Geology, 28: 707-710.

Jahnke, L.L., Embaye, T., Hope, J., Turk, K.A., Van Zuilen, M., Des Marais, D.J., Farmer, J.D. \& Summons, R.E. (2004): Lipid biomarker and carbon isotopic signatures for stromatolite-forming, microbial mat communities and Phormidium cultures from Yellowstone National Park.- Geobiology, 2: 31-47.

Jankowski, B. (1981): die Geschichte der Sedimentation im Nördlinger Ries und Randecker Maar.- Bochumer geologische und geotechnische Arbeiten, 6, $15 \mathrm{~S}$.

Kalkowsky, E. (1908): Oolith und Stromatolith im norddeutschen Buntsandstein.- Zeitschrift der Deutschen Geologischen Gesellschaft, 60: 68-125.

Kandler, O. (1981): Archaebakterien und Phylogenie der Organismen.- Naturwissenschaften, 68: $183-192$.

Kazmierczak, J. \& Kempe, S. (2004): Microbialite formation in seawater of increased alkalinity, Satonda Crater Lake, Indonesia - Discussion.- Journal of Sedimentary Research, 74: 314-317.

Kelts, K. \& Hsü, K.J. 1978): Freshwater carbonate sedimentation.- [In:] Lerman, A. (Ed.): Lakes. Chemistry. Geology. Physics: 295-323, Springer, New York.

Kelts, K. (1988): Environments of depositions of lacustrine petroleum source rocks: an introduction.- [In]: Fleet, A.J., Kelts, K. \& Talbot, M.R. (Eds.): Lacustrine Petroleum Source Rocks.- Geological Society Special Publication, 40: 3-26. 
Kempe, S. (1990): Alkalinity: The link between anaerobic basins and shallow water carbonates? - Naturwissenschaften, 77: 426-427.

Kempe, S. \& Degens, E.T. (1985): An early soda ocean? - Chemical Geology, 53: 95-108.

Kempe, S. \& Kazmierczak, J. (1990a): Calcium carbonate supersaturation and the formation of in situ calcified stromatolites.- [In:] Ittekot, V.A., Kempe, S., Michaelis, W. \& Spitz, A. (Eds.): Facets of Modern Biogeochemistry: 255-278, Springer, Berlin.

Kempe, S. \& Kazmierczak, J. (1990b): Chemistry and stromatolites of the sea-limked Satonda Crater Lake, Indonesia: A recent model for the Precambrian sea? - Chemical Geology, 81: 299-310.

Kempe, S. \& Kazmierczak, J. (1993): Satonda Crater Lake, Indonesia: Hydrogeochemistry and Bicarbonates.- Facies, 28: 1-32.

Kempe, S. \& Kazmierczak, J. (1994): The role of alkalinity in the evolution of the ocean chemistry, organization of living systems, and biocalcification processes.- Bulletin de l'Institut océanographique Monaco, no. spec., 13: 61-117.

Kempe, S., Kazmierczak, J., Landmann, G., Konuk, T., Reimer, A. \& Lipp, A. (1991): Largest known microbialites discovered in Lake Van, Turkey.- Nature, 349: 605-608.

Kent, D. B. \& Kastner, M. (1985): $\mathrm{Mg}^{2+}$ removal in the system $\mathrm{Mg}^{2+}$-amourphous $\mathrm{SiO}_{2}-\mathrm{H}_{2} \mathrm{O}$ by adsorption and Mg-hydroxysilicate precipitation. Geochimica et Cosmochimica Acta, vol. 49, 1123-1136, Pergamon Press, Oxford.

Keupp, H. \& Arp, G. (1990): Aphotische Stromatolithe aus dem süddeutschen Jura (Lias, Dogger).- Berliner Geowissenschaftliche Abhandlungen, A, 124: 3-33.

Knauth, L.P. (1998): Salinity history of the earth`s early ocean. -Nature, 395, 6702: 554.

Knoll, A.H. \& Golubic, S. (1992): [In:] Schidlowski, M.; Aharon \& Golubic, S. (Eds.): Early Organic Evolution: 450-462, Springer, Heidelberg.

Knoll, A.H., Fairchild, I.J. \& Swett, K. (1993): Calcified microbes in Neoproterozoic carbonates: Implications for our understanding of the Proterozoic/Cambrian Transition.Palaios, 8: 512-525.

Konhauser, K.O. \& Ferris, F.G. (1996): Diversity of iron and silica precipitation by microbial mats in hydrothermal waters, Iceland: Implications for Precambrian iron formations.Geology, 24: 323-326.

Konhauser, K.O., Phoenix, V.R., Bottrell, S.H., Adams, D.G. \& Head, I.M. (2001): Microbialsilica interactions in Iceland hot spring sinter: possible analogues for some Precambrian siliceous stromatolites.- Sedimentology, 48: 415-433.

Konishi, Y., Prince, J. \& Knott, B. (2001): The fauna of thrombolitc microbialites, Lake Clifton, Western Australia.- Hydrobiologia, 457: 39-47.

Kretschmar, M. (1982): Fossile Pilze in Eisen-Stromatolithen von Warstein (Rheinisches Schiefergebirge).- Facies, 7: 237-260.

Lowe, D.R. (1994): Abiological origin of described stromatolites older than 3,2 Ga. Geology, 22: $387-390$.

Lowenstam, H.M. \& Weiner, S. (1989): On biomineralization.- 324 p., Oxford University Press, Oxford.

Lowenstein, T.K., Timofeeff, M.N., Brennan, S.T., Hardie, L.A. \& Demicco, R.V. (2001): Oscillations in Phanerozoic seawater chemistry; evidence from fluid inclusions.- Science, 294 (5544): 1086-1088. 
Macintyre, IG., Prufert-Bebout, L. \& Reid, R. P. (2000): The role of endolithic cyanobacteria in the formation of lithified laminae in Bahamian stromatolites.- Sedimentology, 47: 915921.

McNamara, K. (1992): Stromatolites.- Western Australian Museum, 27 S., Perth.

Merz, M.U.E. (1992): The biology of carbonate precipitation by cyanobacteria.- Facies, 26: 81102.

Merz-Preiß, M.U.E. \& Riding, R. (1999): Cyanobacterial tufa calcification in two freshwater streams: ambient environment, chemical thresholds and biological processes. Sedimentary Geology, 126: 103-124.

Moore, L.S. (1987): Water chemistry of the coastal saline lakes of the Clifton-Preston lakeland system, South-western Australia, and its influence on stromatolite formation.- Australian Journal of Marine and Freshwater Research 38: 647-660.

Moore, L.S. (1993): The modern microbialites of Lake Clifton, south-western Australia. - PhD Thesis Department of Microbiology University of Western Australia.

Moore, L.S., Knott, B. \& Stanley, N. (1983): The stromatolites of Lake Clifton, Western Australia. - Search 14: 309-314.

Moore, L.S. \& Burne, R.V. (1994): The modern thrombolites of Lake Clifton, Western Australia.- [In:] Bertrand-Sarfati, J. \& Monty, C. (Eds.): Phanerozoic stromatolites II, 329, Kluwer Academic Publications.

Neuweiler, F., Gautret, P., Thiel, V., Lange, R., Michaelis, W \& Reitner, J. (1999): Petrology of Lower Cretaceous carbonate mud mounds: insights into organomineralic deposits of the geological record.- Sedimentology, 46: 837-859.

Nichols, P.D., Volkman, J.K., Palmisane, A.C., Smith, G.A. \& White, D.C. (1988): Occurrence of an isoprenoid $\mathrm{C}_{25}$ diunsaturated alkene and high neutral lipid content in antarctic seaice diatom communities.- Journal of Phycology, 24: 90-96.

Nordstrom, D.K.; Plummer, L.N.; Wigley, T.M.L, Wolery, T.J., Ball, J.W., Jenne, E.A., Bassett, R.L., Crerar, D.A., Florence, T.M., Fritz, B., Hoffmann, M., Holdren, G.R., Jr., Lafon, G.M., Mattigod, S.V., McDuff, R.E., Morel, F., Reddy, M.M., Sposito, G. \& Thrailkill, J. (1979): A comparison of computerized chemical models for equilibrium calculations in aqueous systems. In: Jenne, E.A.(ed.): Chemical Modeling in Aqueous Systems, Specification, Sorption, Solubility, and Kinetics.- American Chemical Society, Symposium Series, 93: 857-892.

Ourisson, G., Albrecht, P. \& Rohmer, M. (1979): The hopanoids.- Pure \& Appl. Chemistry, 51: 709-729.

Pache, M., Reitner, J. \& Arp, G. (2001): Geochemical evidence for the formation of a large Miocene „Travertine“ mound at a sublacustrine spring in a soda lake (Wallerstein Castle Rock, Nördlinger Ries, Germany.- Facies, 45: 211-230.

Paerl, H.W., Steppe, T.F. \& Reid, R.P. (2001): Bacterially mediated precipitation in marine stromatolites.- Environmental Microbiology, 3: 123-130.

Parkhurst, D.L. \& Appelo, C.A.J. (1999): User`s guide to PHREEQC (version 2)- a computer program for specification, one-dimensional transport, and inverse geochemical calculations. U.S. Geological Survey, Water-Resources Investigations, Report 99-4259, $312 \mathrm{~S}$. 
Paull, C.K., Chanton, J.P., Neumann, A.C., Coston, J.A. \& Martens, C.S. (1992): Indicators of methane-derived carbonates and chemosynthetic organic carbon deposits: examples from the Florida Escarpment.- Palaios, 7: 361-375.

Pentecost, A. \& Riding, R. (1986): Calcification in cyanobacteria. [In]: Leadbeater, B.S.C. \& Riding, R. (Eds.): Biomineralization in lower plants and animals: 73-90, Clarendon Press, Oxford.

Playford, P. E. (1988): Guidebook to the geology of Rottnest Island.- Geological Society of Australia. Western Australian Division Excursion Guidebook no. 2, 67 S.

Reid, P.R., MacIntyre, I.G., Browne, K.M., Steneck, R.S. \& Miller, T. (1995): Modern marine stromatolites in the Exuma Cays, Bahamas: uncommonly common.- Facies, 33: 1-17.

Reid, R.P., Visscher, P.T., Decho, A.W., Stolz, J.F., Bebout, B.M., Dupraz, C., Macintyre, I.G., Paerl, H.W., Pinkey, J.L., Prufert-Bebout, L., Steppe, T.F., DesMarais, D.J. (2000): The role of microbes in accretion, lamination and early lithification of modern marine stromatolites.- Nature, 406,6799: 989-992.

Reid, R.P., James, N.P., Macintyre, I.G., Dupraz, C.P. \& Burne, R.V. (2003): Shark Bay stromatolites: Microfabrics and reinterpretation of origins. - Facies, 49: 299-324.

Reitner, J. (1993): Modern cryptic microbialite/metazoan facies from Lizard Island (Great Barriere Reef, Australia) formation and concepts.- Facies, 29: 2-14.

Reitner, J. Neuweiler, F., Flajs, G., Vigener, M., Keupp, H., Meischner, D., Paul, J., Warnke, K., Weller, H., Dingle, P., Hensen., C., Schäfer, P., Gautret, P., Leinfelder, R.R., Hüssner, H \& Kaufmann, B. (1995): Mud Mounds: a polygenetic spectrum of fine-grained carbonate build-ups.- Facies, 32: 1-70.

Reitner, J., Paul, J, Arp, G. \& Hause-Reitner, D. (1996): Lake Thetis domal microbialites - a complex framework of calcified biofilms and organomicrites (Cervantes, Western Australia). In: Reitner, J., Neuweiler, F. \& Gunkel, F, (Eds.): Global and Regional Controls on Biogenic Sedimentation. I. Reef Evolution. Research Reports.- Göttinger Arbeiten Geologie und Paläontologie, Sb2, 85.89.

Renaut, R.W., Jones, B. \& Tiercelin, J.-J. (1998): Rapid in situ silification of microbes at Loburu hot springs, Lake Bogoria, Kenya Rift Valley.- Sedimentology, 45, 6: 1083-1103.

Riding, R. (1977): Calcified Plectonema (blue-green algae), a recent example of Girvanella from Aldabra Atoll.- Palaeontology, 20: 33-46.

Riding, R. (1982): Cyanophyte calcification and changes in ocean chemistry.- Nature, 299: 814-815.

Riding, R. (1991): Classification of microbial carbonates. [In:] Riding, R. (Ed.): Calcareous Algae and Stromatolites: 21-51, Springer, Berlin.

Riding, R. (1992): Palaeoclimatology; the algal breath of life.- Nature, 359: 13-14.

Riding, R. (1993): Phanerozoic patterns of marine $\mathrm{CaCO}_{3}$ precipitation.- Naturwissenschaften, 80: 513-516.

Riding, R. (1994): Evolution of algal and cyanobacterial calcification .- [In:] Bengtson, S. (Ed.): Early life on Earth. -Nobel Symposium, 84: 426-438, Columbia University Press, New York.

Riding, R. \& Liang, L. (2005): Geobiology of microbial carbonates : metazoan and seawater saturation state influence on secular trends dirung the Phanerozoic.- Palaeogeography, Palaeoclimatology, Palaeoecology, 219: 101-115. 
Robinson, N. \& Eglinton, G. (1990): Lipid chemistry of Iceland hot spring microbial mats.Organic Geochemistry, 15: 291-298.

Rohmer, M, Bouvier-Nave, P., Ourisson, G. (1984): Distribution of hopanoid Triterpenes in Prokaryotes.- Journal of General Microbiology, 130: 1137-1150.

Rothe, P., Hoefs, J. \& Sonne, V. (1974): The isotopic composition of Tertiary carbonates from the Mainz Basin: an example of isotopic fractionation in 'closed basins`.- Sedimentology, 21: 373-395.

Rowland, S.J. \& Robson, J.N. (1990): The widespread occurrence of highly branches acyclic $\mathrm{C}_{20}, \mathrm{C}_{25}$ and $\mathrm{C}_{30}$ hydrocarbons in recent sediments and biota-a review.- Marine Environmental Research, 30: 191-216.

Schopf, J.W. (1968): Microflora of the Bitter Springs Formation. -Journal of Paleontology, 42: 651-688.

Schopf, J.W. (1993): Microfossils of the early Archaen Apex Chert; new evidence of the antiquity of life.- Science 260, 5108: 640-646.

Sebag, D., Verrechia, E.P., Seong-Joo Lee \& Durand, A. (2001): The natural hydrous sodium silicates from the nothern bank of Lake Chad: occurence, petrology and genesis.Sedimentary Geology, 139: 15-31.

Shiea, J., Brassell, S.C. \& Ward, D. M. (1990): Mid-chain branched mono- and dimethyl alkanes in hot spring cyanobakterial mats: A direct biogenic source for branched alkanes in ancient sediments?- Organic Geochemistry, 15, 3: 223-231.

Siever, R. (1992): The silica cycle in the Precambrian.- Geochimica et Cosmochimica Acta, 56, 8: 3265-3272.

Souza-Egipsy, V., Wierzchos, J., Ascaso, C. \& Nealson, K. H. (2005): Mg-Silica precipitation in fossilization mechanisms of sand tufe endolithic microbial community, Mono Lake (California).- Chemical Geology, 217: 77-87.

Spencer, R.J., Eugster, H.P. \& Jones, B.F. (1985): Geochemistry of Great Salt Lake, Utah II: Pleistocene-Holocene evolution.- Geochimica et Cosmochimica Acta, 49: 739-747.

Stumm, W. \& Morgan, J.J. (1996): Aquatic Chemistry. Chemical equilibria and rates in natural waters. 3rd Ed., 1022 S., Wiley, New York.

Summons, R.E., Jahnke, L.L, Hope, J. M. \& Logan, G.A. (1999): 2-Methylhopanoids as biomarkers for cyanobacterial oxygenic photosynthesis.- Nature, 400: 554-557.

Thiel, V. (1997): Organische Verbindungen in Porifera und biogenen Carbonaten: Fazies, Chemotaxonomie und molekulare Fossilien.- Dissertation, Universität Hamburg.

Thiel, V., Merz-Preiß, M., Reitner, J. \& Michaelis, W. (1997): Biomarker studies on microbial carbonates: extractable lipids of a calcifying cyanobacterial mat (Everglades, USA).Facies, 36: 163-172.

Thiel, V., Jenisch, A, \& Wörheide, G., Löwenberg, A., Reitner, J. \& Michaelis, W. (1999): Mid-chain branched alkanoic acids from „living fossil“ desmosponges: a link to ancient sedimentary lipids?- Organic Geochemistry, 30: 1-14.

Tissot, B.P. \& Welte, D.H. (1984): Petroleum formation and occurrence. 699 S., Springer, New York.

Trichet, J. \& Défarge, C. (1995): Non-biologically supported organomineralization.- Bulletin de l'Institut océanographique Monaco, no. spéc., 14: 203-236. 
Trichet, J., Défarge, C., Tribble, J., Tribble, G. \& Sansone, F. (2001): Christmas Island lagoonal lakes, models for the deposition of carbonate-evaporite-organic-laminated sediments.- Sedimentary Geology, 140, 1-2: 177-189.

Usdowski E., Hoefs, J. \& Menschel, G. (1979): Relationship between ${ }^{13} \mathrm{C}$ and ${ }^{18} \mathrm{O}$ fractonation and changes in major element composition in a recent calcite-depositing spring - A model of chemical variations with inorganic $\mathrm{CaCO}_{3}$ precipitation.- earth and Planetary Science Letters, 42: 267-276.

van Gemerden, H. (1993): Microbial mats: a joint venture.- Marine Geology, 113: 3-25.

Visscher, P.T., Reid, R.P. \& Bebout, B.M. (2000): Microscale observations of sulfate reduction: Correlation of microbial activity with lithified micritic laminae in modern marine stromatolites.- Geology, 28: 919-922.

Volkman, J.K., Barrett, S.M. \& Dunstan, G.A. (1994): $\mathrm{C}_{25}$ and $\mathrm{C}_{30}$ highly branches isoprenoid alkenes in laboratory cultures of two marine diatoms.- Organic Geochemistry, 21: 407413.

Walter, M.R. \& Heys, G.R. (1985): Links between the rise of the metazoa and the decline of stromatolites.- Precambrian Research, 29: 149-174.

Walter, M.R. (1994): Stromatolites: The main geological source of information on the evolution of the early benthos.- [In:] Bengtson, S. (Ed.): Early Life on Earth.- Nobel Symposium, 84: 270-286, Columbia University Press, New York.

Wang, R., Brassell, S.C., Fu, J. \& Sheng, G. (1998): Molecular indicators of microbial contributions to recent and Tertiary hypersaline lacustrine sediments in China.- [In]: Zheng Mianping, Hurlbert, S.H. \& Williams, W.D. (Eds.): Saline Lakes VI. Hydrobiologia, 381: 77-103, Kluwer Academic Publishers.

Wee, N., Phoenix, V.R., Konhauser, K.O., Benning, L.G. \& Ferris, F.G. (2003): The effect of cyanobacteria on silica presipitation at neutral $\mathrm{pH}$; implications for bacterial silification in geothermal hot springs.- Chemical Geology, 199: 83-90.

White, D.C., Smith, G.A., Gehron, M..J., Parker, J.H., Findley, R.H., Martz, R.F. \& Fredrickson, H.L. (1983): he groundwater aquifer microbiota: biomass, community structure, and nutritional status.- Developments in Industrial Microbiology, 24: 201-211.

Woo, K.S., Lee, K.C., Khim, B.K. \& Yoon, H.S. (2003): Paleosalinity and paleoproductivity fluctuations recorded in the Cretaceous lacustrine stromatolites, Gyeongsang Basin, Korea (Abstract). - Geological Society of America Annual Meeting, paper No. 207-1.

Zeng, Y.B., Ward, D.M., Brassell, S.C. \& Eglinton, G. (1992a): Biogeochemistry of hot spring environments 2. Lipid compositions of Yellowstone (Wyoming, U.S.A.) cyanobacterial and Chloroflexus mats.- Chemical Geology, 95: 327-345.

Zeng, B.Y., Ward, D.M., Brassell, S.C. \& Eglinton, G. (1992b): Biogeochemistry of hot spring environments 3. Apolar and polar lipids in the biologically active layers of a cyanobacterial mat.- Chemical Geology, 95: 347-360. 


\section{Danksagung}

Herrn Prof. Joachim Reitner danke ich für die Vergabe des Promotionsthemas und die Betreuung dieser Arbeit. Prof. Volker Thiel danke ich für das Korreferat. Beiden gilt mein Dank für ihr Interesse, ihre hilfreichen Tipps und Kommentare.

Dr. Gernot Arp danke ich für sein Interesse an der Arbeit, sein offenes Ohr, für sein umfangreiches Wissen, seine Gegenfragen und Erklärungen, für die Einweisungen in Adobe Photoshop und PHREEQC, seine Hilfestellung beim Mikroskopieren und bei den EMS-Messungen sowie für die Sichtung des Manuskriptes und seine hilfreichen Kommentare.

Dr. Andreas Reimer danke ich für sein Interesse, für die gemeinsame Gelände-Kampagne 2002, die Hilfe im Labor und bei der Auswertung der hydrochemischen Daten, für die Einweisung in PHREEQC sowie für die Hilfe bei allen nur denkbaren Sigma-Plot- und Excel-Problemen, für die Sichtung des Manuskriptes und die hilfreichen Kommentare.

Prof. Joachim Reitner, Dr. Gernot Arp und Dr. Andreas Reimer waren so freundlich, die Proben der Geländekampagne 1998 für diese Arbeit zur Verfügung zu stellen.

Für die Unterstützung im Gelände und im Labor möchte ich Dipl.-Geol. Sven Knollmann danken. Frau Ulrike Meliß gilt mein Dank für die Einweisung in die Biomarker-Aufbereitung. Frau Birgit Röhrig danke ich für die Hilfestellungen im Labor. Herrn Wolfgang Dröse und Herrn Alexander Satmari danke ich für die Hilfe bei der histologischen Einbettungen sowie der Herstellung der Dünnschnitte und Dünnschliffe.

JProf. Jörn Peckmann danke ich für die Auswertung der GC-MS-Chromatogramme. Dr. A. Kronz danke ich für seine Hilfe bei den EMS-Messungen. Prof. K.-H. Nitsch danke ich für die Messungen und Auswertungen zur Röntgendiffraktometrie.

Dipl.-Geol. Cathrin Hühne, Dipl.-Geol. Stefan Delecat und Wolfgang Dröse danke ich die physische wie auch psychische Unterstützung. Cathrin Hühne danke ich für den EMS-Tag. Dr. Thomas Bode danke ich für die Hilfe bei Computerproblemen.

Ich danke dem Department of Conservation and Land Management (CALM) in Perth für die Möglichkeit, die Proben an den zum Teil in Nationalparks gelegenen Seen nehmen zu dürfen.

Ich danke der DFG für die 2⿺辶⿸厃㔾丷-jährige Finanzierung in dem Projektes Re 665/18-1 und 18-2, Projektleiter Prof. J. Reitner): Biofilme, Makromoleküle und organische Restsubstanzen als Matrizen bei der Bildung von Organo- und Biomineralen - Geobiologische Faktoren bei der Evolution der Biomineralisation.

Ich danke meinen Eltern und meiner Schwiegermutter ganz herzlich für das Babysitten und für ihre beständige, liebevolle Unterstützung. Unermesslicher Dank gilt meinem Mann Bentick für seine liebevolle und geduldige Unterstützung, das Reduzieren der Schreib- und Kommafehler und sein Verständnis. 
Anhang 


\section{Tafel 1}

\section{Subfossile Stromatolithe, Lake Thetis}

Fig. A: Interlitorale Stromatolithe des S-Ufers. Es zeigt sich eine Tendenz zu seitlich zusammengewachsenen Biohermen. Die Stromatolithe sitzen einem proximal abtauchenden Karbonatsaum auf.

Fig. B: Interlitoraler Stromatolith mit nicht-erodiertem Topbereich, der eine knotige Oberfläche aufweist, welche im Zentrum weiß gefärbt ist (Zone I) und zum Schulterbereich in grün bis dunkelgraue Knötchen übergeht (Zone II). Direkt am Wasserkontakt befindet sich der Übergang zu einem kranzartigen Karbonatsaum um die Biohermbasis (Zone III), der beige orange bis dunkel orange im unteren Teil gefärbt ist. Die Wassertiefe während der Trockenzeit beträgt 10-15 cm. In der Regenzeit ist dieser Stromatolith vollständig wasserbedeckt.

Fig. C: Stromatolith (S-Ufer) an der Wassergrenze mit stark erodiertem Topbereich. In violetter Farbe ist die eingespülte „flocculent mat“ (reich an SPB) zu erkennen Zwischen äußerster Kruste und Zentrum ist der Zwischenbereich erodiert. Der Durchmesser des Stromatolithes beträgt ca. $75 \mathrm{~cm}$, die Höhe $90 \mathrm{~cm}$.

Fig. D: Erodierte Stromatolithe am S-E-Ufer. Aufgrund der fortgeschrittenen Erosion zeigt sich ein konzentrischer Aufbau von ebenmäßigen Karbonatkrusten auf einem Kernbereich mit glatter Oberfläche.

Fig. E: Fossiler Stromatolith (S-Ufer, Erosionsfläche) mit glatten, weißen Oberflächen. Durch vertikale Klüfte wird der Stromatolith in polygonal-begrenzte, zum Top hin abgerundete „Kissen“-Strukturen unterteilt. Der Maßstab ist $30 \mathrm{~cm}$ lang.

Fig. F: Überblick des E-Ufers. Wie auch am N-Ufer besteht der Bereich des Interlitorals aus Karbonat-Sand und -Blöcken, die stark erodiert und eingeebnet sind und nur z.T. einen konzentrischen Aufbau erkennen lassen.

Fig. G: Oberste Stromatolith-Kruste mit deutlicher Laminierung. Beachte den Wechsel von hellen Lagen (Aragonit mit reliefausgleichenden „Organomikrit“-Krusten als Hohlraumfüllungen und Scytonema-Phormidium-Lagen) und dunklen Einschaltungen (Entophysalis-Lagen). Die letzte Phase wir von einem feinlaminierten, hellen „Organomikrit“ bebildet. Probe LTh 98/13 (siehe auch Abb. 4, 9 und 10)

Fig. H: Aufsicht auf die knotige Oberfläche von Fig. G. Probe LTh 98/13. 
Tafel 1
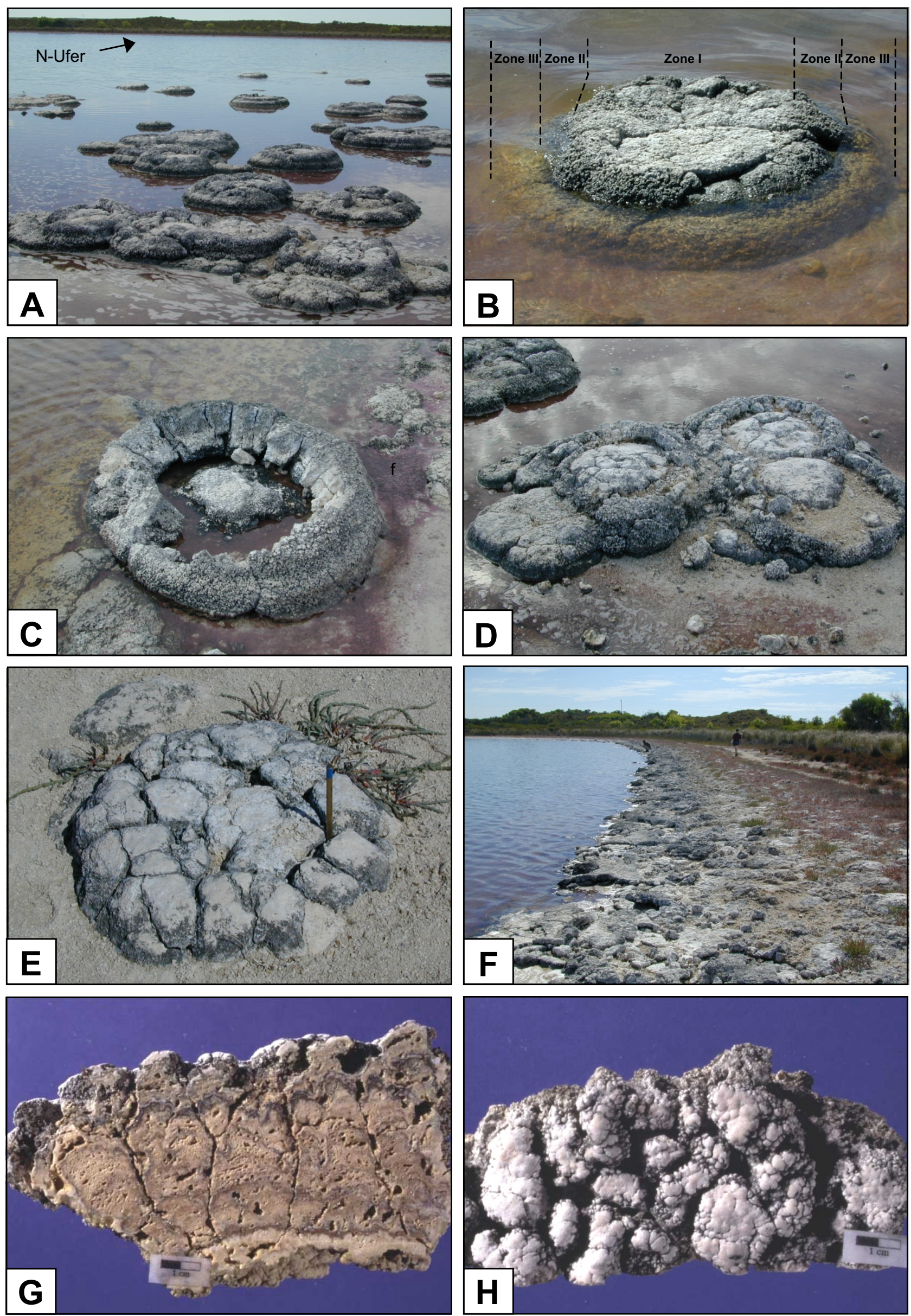


\section{Tafel 2}

\section{Subfossile Stromatolithe, Lake Thetis}

Fig. A: Aragonitische Scytonema-Phormidium-Lage. Die Scytonema-Filamente sind in erekter Position von krümeligem Aragonit umgeben. In der rechten Bildhälfte ist ein büschelartiges Wachstum deutlich. Zum Top folgt eine dunkel-pigmentierte Entophysalis-Lage mit anschließender „Organomikrit“-Kruste.

Dünnschliffaufnahme, Hellfeld, LTh 98/13.

Fig. B: Kolonie-artige Ansammlung kokkaler Cyanobakterien-Relikte unterhalb einer abschließenden „Organomikrit“-Kruste. Hellfeld. Probe LTh 98/14.

Fig. C: Dünnschliffaufnahme, Hellfeld. Probe LTh 98/13. Deutlich zu erkennen sind dunkel gefärbte Entophysalis-Kolonien (E), die unterhalb des „Organomikrit“ (O) des Tops, aber auch in dessen Vertiefungen und Buchten vorkommen. ScytonemaFilamente (S) zeigen eine orange-gelbliche Färbung und erektes Wachstum. Sedimentäre Taschenfüllungen $(\mathrm{T})$ beinhalten Peloide, holozänen Schill und Extraklasten. Dünnschliffaufnahme, Hellfeld. Probe LTh 98/13.

Fig. D: Eine amorphe $\mathrm{Mg}-\mathrm{Si}$-Phase zeigt unter gekreuzten Polarisatoren besonders deutlich, wie die Übergänge zu dem Aragonit diffus verlaufen. Im Zentrum der Phase zeigen sich durch Dehydrierung der ehemals wasserreichen Phase deutliche Schrumpfungsrisse, welche nicht auf den umliegenden Aragonit übergehen. Gekreuzte Polarisatoren. Probe LTh 98/14.

Fig. E-F: Häufig bildet sie amorphe Mg-Si-Phase Bereiche zwischen dem Aragonit und den laminierten „Organomikrit“-Krusten. Auffallend ist die orange-gelbe Färbung. Übergänge zu den Aragoniten verlaufen diffus bis schlierig. Im Kontakt zu den „Organomikrit“-Krusten ist der Kontakt scharf. Zwischen gekreuzten Polarisatoren erscheint die Phase in Auslöschung. Die „Organomikrit“-Krusten zeigen einen radialfibrösen Zement in den Zwischenlagen. Fig. E: Hellfeldaufnahme. Fig. F: gekreuzte Polarisatoren. Probe LTh 98/14.

Fig. G: Die amorphe Mg-Si-Phase innerhalb des Aragonites. Je nach Durchmischung mit dem Aragonit zeigen sich unterschiedlich ausgeprägt Stufen der Auslöschung zwischen gekreuzten Polarisatoren. In wenig durchmischten Bereichen zeigt die Mg-Si-Phase deutliche Schrumpfungsrisse und Auslöschung. Gekreuzte Polarisatoren. Probe LTh 98/14.

Fig. H: Die mikrokristallinen, klumpigen Aragonite werden von „Organomikrit“-Krusten umschlossen. Dünnschliffaufnahme, Hellfeld. Probe LTh 98/14. 


\section{Tafel 2}
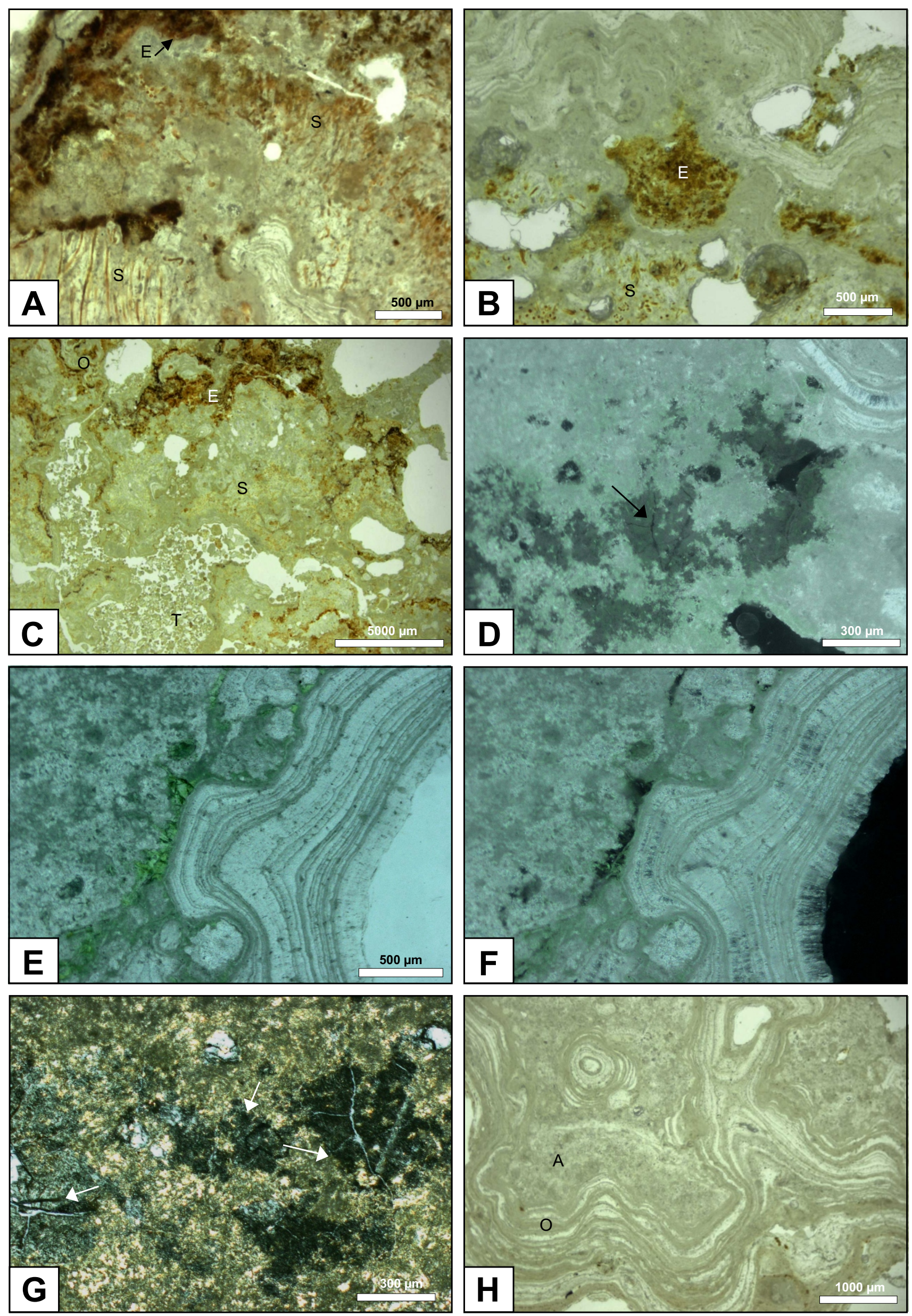


\section{Tafel 3}

\section{Rezente Biofilme, Lake Thetis}

Fig. A-B: Rezenter Biofilm mit Scytonema-Filamenten. Die Aragonitpräzipitate haben keinen lokalen Bezug zu den Cyanobakterien-Filamenten. B: Aufnahme mit gekreuzten Polarisatoren LTh 98/5.

Fig. C: Scytonema-Filament mit scheinbarer Verzweigung und angrenzender GloeocapsaKolonie. Probe LTh 98/5.

Fig. D: Scytonema-Filamente. Die Polysaccharid-Scheiden sind karbonatisch imprägniert. Probe LTh 98/5.

Fig. E-H: Kalzifizierte und unkalzifizierte Scytonema-Lagen. Die basale Lage repräsentiert karbonatisch eingebettete Filamente und Scheiden. Die darauffolgende, äußere Lage der Scytonema-Filamente ist komplett unkalzifiziert und stellt den intakten Biofilm dar. Fig. E: Hellfeldaufnahme. Fig. F: UV-Anregung (Zeiss 01) und polarisiertes Licht. Fig. G: Aufnahme mit DIC. Fig. H: Gekreuzte Polarisatoren. Probe LTh 98/5. 
Tafel 3
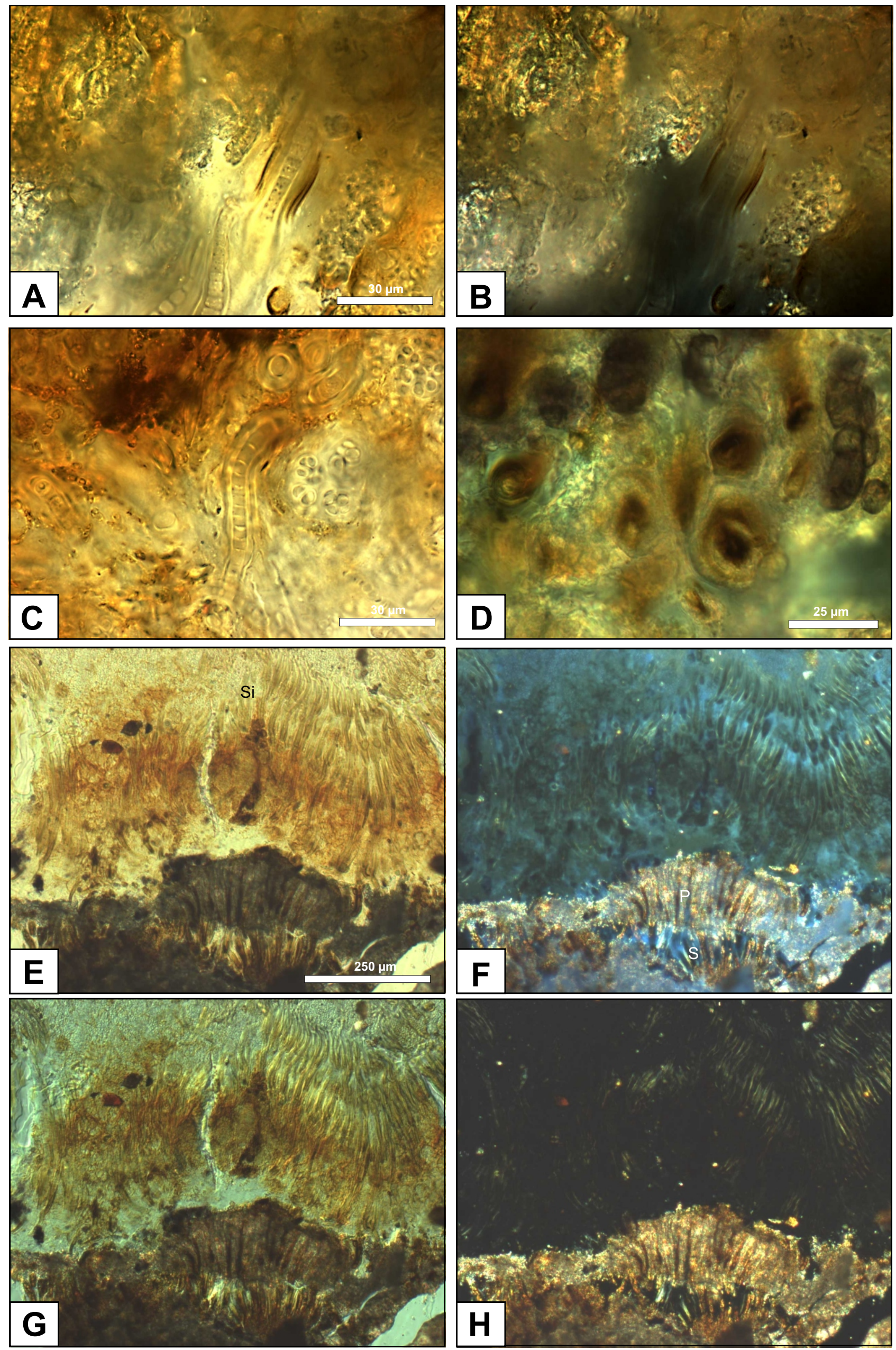


\section{Tafel 4}

\section{Rezente Biofilme, Lake Thetis}

Fig. A: Im äußeren Bereich sind Diatomeen-Schalen häufig mit senkrechter Orientierung zur Karbonat-, bzw. Biofilm-Oberfläche angeordnet. Der mittlere Teil ist von Gloeocapsa-Kolonien charakterisiert. Scytonema-Filamente sind an der Karbonatoberfläche angeheftet, zeigen aber keine Verkalkungsstrukturen innerhalb der Polysaccharid-Scheiden. Probe LTh 98/5.

Fig. B-C: Hellfeldaufnahme eines Calcein- gefärbten Biofilm, der von kokkalen Cyanobakterien $(\mathrm{kC})$ dominiert wird. Die Vorkommen ,frischer“" Aragonitpräzipitate $(\mathrm{P})$ sind regellos verteilt und nicht an den Biofilm gekoppelt. Fig. C: Aufnahme mit gekreuzten Polarisatoren. Probe LTh 02/3.

Fig. D: Mikrokristalliner Aragonit als Basis des (mit Calcein gefärbten) Biofilm, der besonders in Hohlräumen und Vertiefungen vorkommt bildet einen „Mikrostromatolith“ als unregelmäßig strukturierte Kruste. Probe LTh 02/3.

Fig. E: Karbonat-imprägnierte Cyanobakterien-Filamentspuren. Kleiner Bildausschnitt aus Fig. D. Probe LTh 02/3.

Fig. F: Diatomeen-Schalen. Hellfeldaufnahme. Probe LTh 02/0.

Fig. G: Rezenter Gloeocapsa-dominierter Biofilm im direkten Kontakt zur Karbonatoberfläche. Hellfeldaufnahme. Probe LTh 02/0. 


\section{Tafel 4}
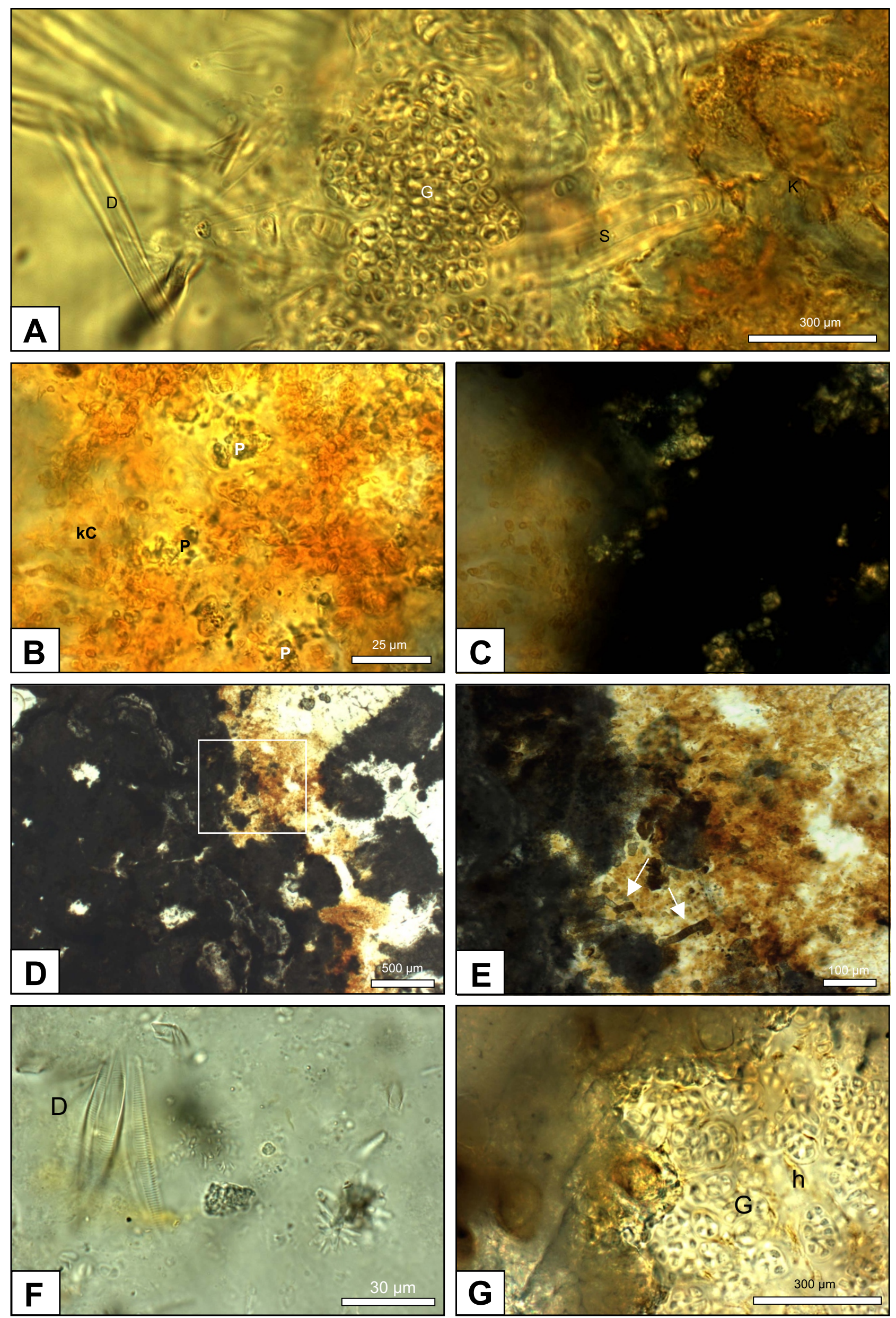


\section{Tafel 5}

\section{Rezente Biofilme, Lake Thetis}

Fig. A: Kokkale Cyanobakterien sind zu fädigen Strukturen angeordnet und repräsentieren vermutlich alterierte Gloeocapsa-Zellen. Hellfeldaufnahme. Probe LTh 02/3.

Fig. B: Gloeocapsa. Hellfeldaufnahme. Probe LTh 02/3.

Fig. C-D: Gloeocapsa-Biofilm an Aragonit-Oberfläche. Durch das polarisierte Licht zeigen sich nicht kalzifizierte Polysaccharid-Scheiden in einem karbonatfreien Biofilm, dessen Umrisse durch frisches Präzipitat nachgezeichnet werden. In Zwickeln der Gloeocapsa-Kolonien sind heterotrophe Bakterien angefärbt. C: Hellfeldaufnahme, Calceinfärbung. D: Gekreuzte Polarisatoren. Probe LTh 02/0.

Fig. E: Karbonatisch eingebettete kokkale Cyanobakterien. Hellfeldaufnahme. Probe LTh $02 / 4$.

Fig. F: Relikte vormals kokkaler Cyanobakterien $(\mathrm{kCR})$ sind in mikrokristalline Karbonatpartikel (K) eingebettet. Hellfeld. Probe LTh 02/3.

Fig. G-H: Biofilm, bestehend aus kokkalen Cyanobakterien (vermutlich abgestorbene Gloeocapsa-Zellen). Fig. G: Hellfeldaufnahme. Fig. H: Karbonatische Fällungsprodukte bestehen nur im Kontaktbereich zu den Cyanobakterien. Polarisiertes Licht. Probe LTh 02/3. 

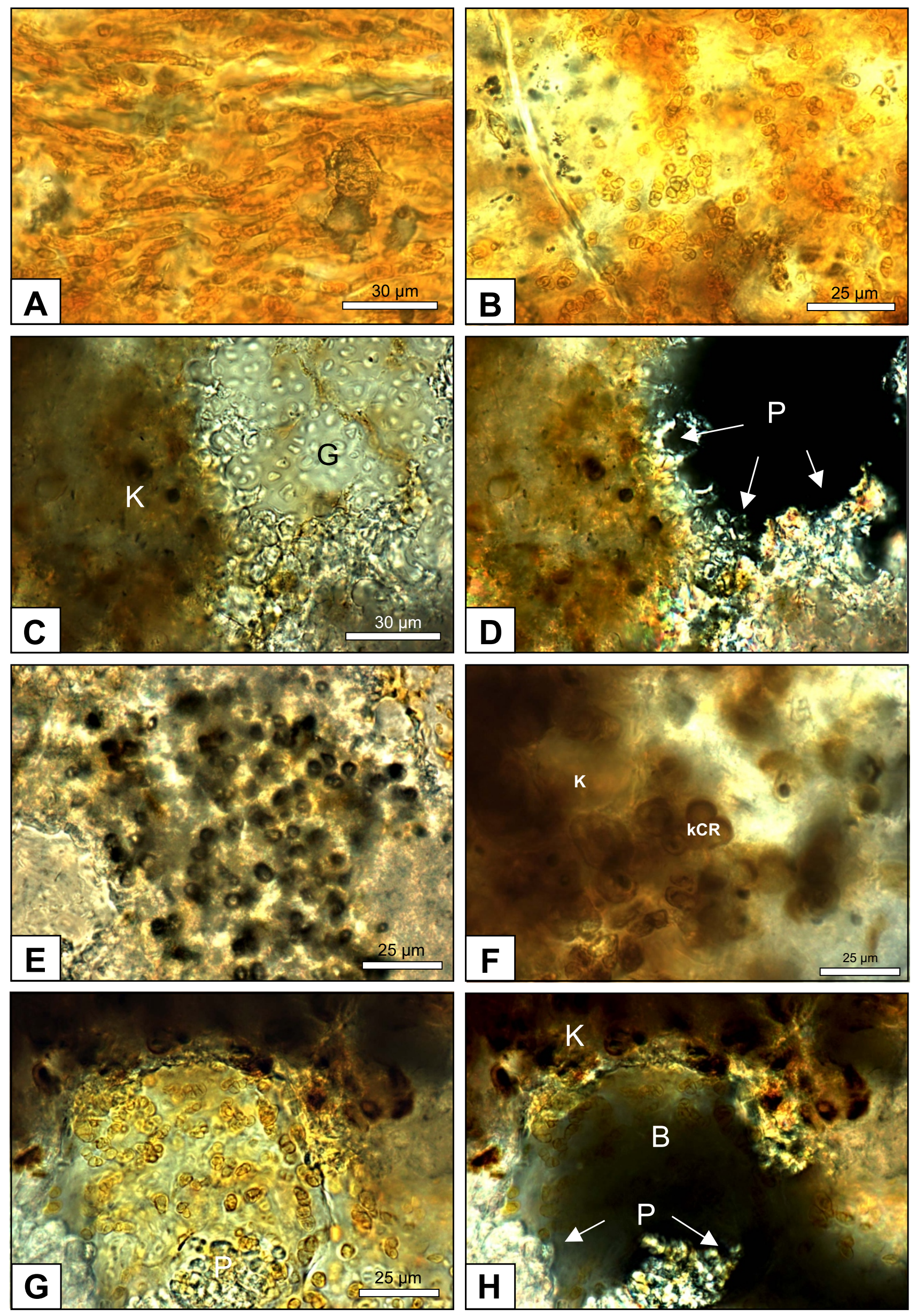


\section{Tafel 6}

\section{Lake Thetis}

Fig. A: Der Biofilm wird von filamentösen Cyanobakterien (Phormidium-Typ) und kokkalen Cyanobakterien aufgebaut. Probe LTh 02/A.

Fig. B: Im Karbonat eingeschlossene Gloeocapsa sp.. Unter UV-Anregung (Zeiss 01) zeigt sich eine deutliche Fluoreszenz, welche sich von der des Karbonates anhebt. Durch das Teilungsmuster können die Kokken als Gloeocapsa sp. interpretiert werden. Probe LTh 02/B.

Fig. C: Diatomeen, Ordnung Centrales (Dc), z.T. im Teilungsstadium. Pennate Diatomeen (Dp) sind aufgrund der Schräglage in der Fokussierebene unscharf. Hellfeldaufnahme. Probe LTh 02/A.

Fig. D: Karbonat-umkrustetes Cyanobakterien-Filament (Phormidium-Morphotyp). Im oberen Teil ist eine Verdickung und unregelmäßige Oberflächenstruktur des Karbonates deutlich zu erkennen. Gekreuzte Polarisatoren. Probe LTh 02/C.

Fig. E-F: Unregelmäßige Aragonit-Klumpen und Gloeocapsa. Fig. E: DIC, Phasenkontrast. Fig. E: unter UV-Anregung (Zeiss 01). Probe Th 02/0.

Bildausschnitt Sowohl die lebenden Zellen, als auch Zwickelbereiche der Kolonie-KontaktStellen weisen eine starke Fluoreszenz auf. Die starke Fluoreszenz geht vermutlich auf heterotrophe Bakterien zurïck. Probe LTh 02/0.

Fig. G: Die ,Entophysalis-Lamelle“ bleibt unter UV-Anregung (Zeiss 01) dunkel, der basale „Organomikrit“ dagegen zeigt besonders innerhalb der Zementzwischenlagen eine starke Fluoreszenz. Letzteres wird als Beleg für enthaltenen Rest-Organik gewertet. Probe LTh 02/B.

Fig. H: Die dislozierte Probe aus dem Sedimentkern besteht aus klumpigen Karbonat- und Organikpartikel in variabler Größenordnung und Bruchstïcken von OstrakodenSchalen. Probe LTh K1 6. 

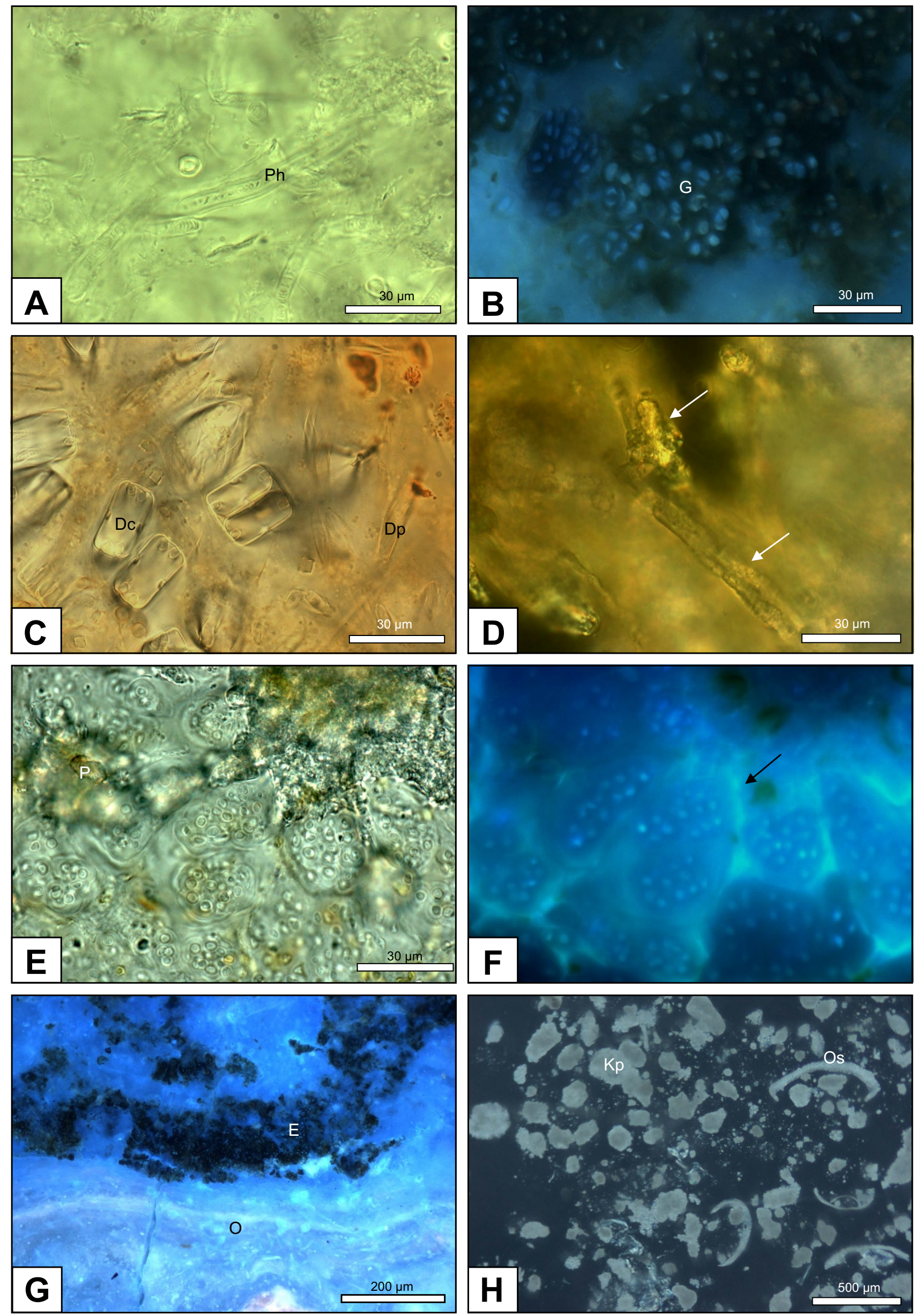


\section{Tafel 7}

\section{Lake Clifton}

Fig. A: Geländeaufnahme des E-Ufers, Blick in Richtung Süden vom Steg aus. Zahlreiche Mikrobialithe säumen den Uferbereich und vereinzeln sich zunehmend in Richtung Seemitte.

Fig. B: Geländeaufnahme, am Steg. Interlitorale Thrombolith-Köpfe liegen dicht beieinander. Zwischen den rundlichen Köpfen des tieferen Interlitorals und der von Seegras begrenzten Überschwemmungebene liegt eine Zone erodierter, stark eingeebneter, fossiler Stromatolithe mit deutlichem konzentrischen Aufbau (Bildausschnitt oben rechts).

Fig. C: Geländeaufnahme, am Steg. Sublitorale Thrombolithe zeichnen sich durch kegelförmige Umrisse und häufig durch ,vent"-artige Öffnungen aus.

Fig. D: Geländeaufnahme, E-Ufer, "mudshore", Überschwemmungsebene mit Karbonatsand- und Schlick auf dessen Oberfläche filigrane, Karbonat-umkrustete Grünalgenbüschel wachsen. Die Wassertiefen der ,ponds“ beträgt nur wenige cm.

Fig. E: Eine weiche Biofilmkruste vom Holzbalken des Steges enthält radialstrahlig aufgebaute Präzipitate, die als inneren Kern braunes, mikrokristallines Material enthalten. Probe LCl 02/12.

Fig. F: Die mikrokristallin umkrustete Grünalgen-Büschel an den "ponds" zeichnen sich durch intern gewachsene Zemente und einer äußeren Umhüllung von DiatomeenSchalen (D) aus. Probe LCL 02/1.

Fig. G: Neben elliptischen Diatomeen sind auch Ansammlungen von Diatomeen der Ordnung Centrales häufig Bestandteil des rezenten Biofilms. Probe LCL 02/10.

Fig. H: Im Kontakt zu der amorphen $\mathrm{Mg}-\mathrm{Si}$-Phase (aSi) und darin eingeschlossenen Filamenten von Cya nobakterien und deren Filamentspuren, zeichnet sich der Biofilm durch unkalzifizierte filamentöse Cyanobakterien (Gattung Scytonema sp. ?) aus. Probe $\mathrm{LCl}$ 02/5. 

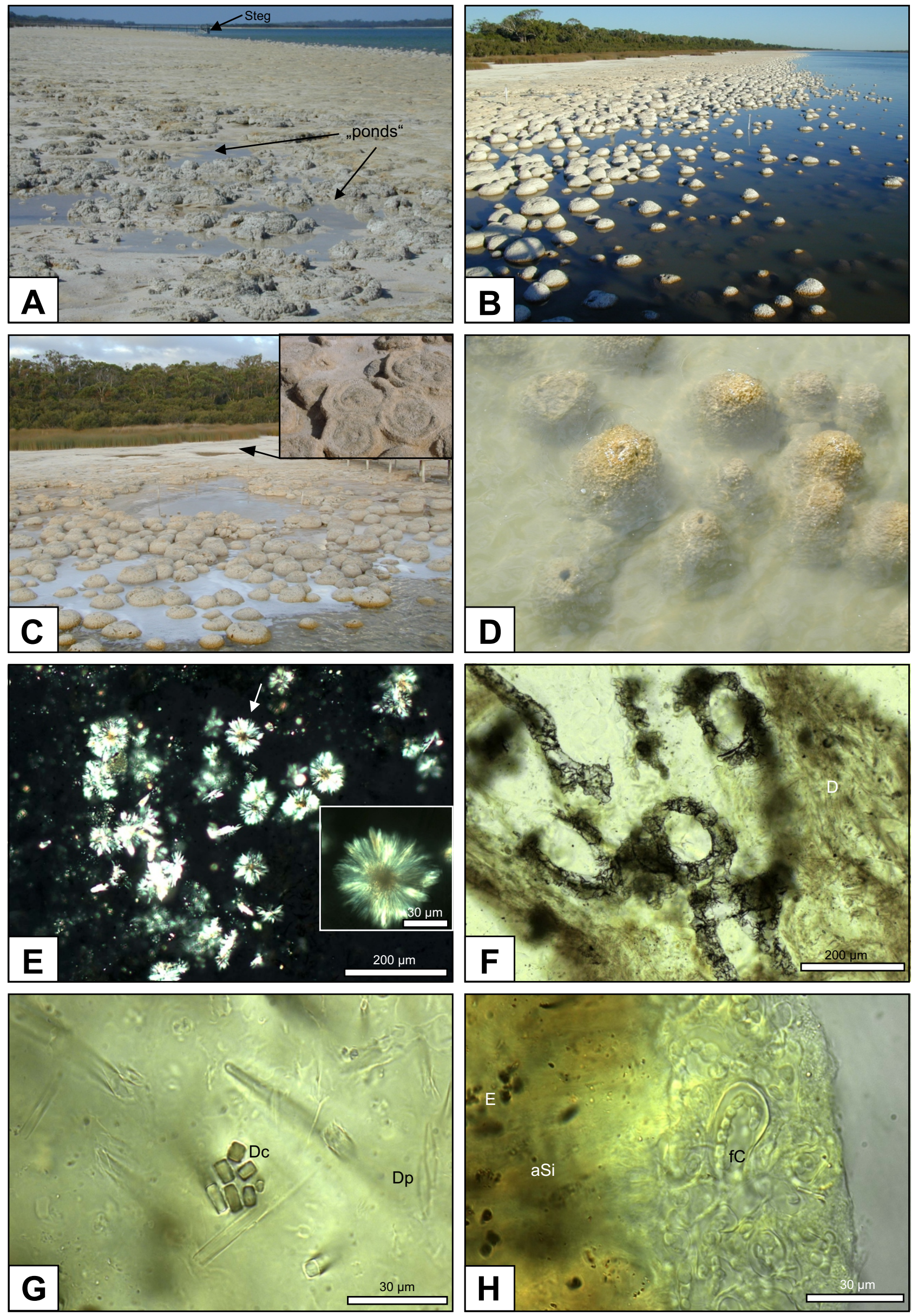


\section{Tafel 8}

\section{Subfossile Thrombolithe und rezente Biofilme, Lake Clifton}

Fig. A: Rezenter Biofilm, bestehend aus Gloeocapsa-Kolonien. Die im Außenbereich dunkel gefärbten Hüllen werden als abgestorbene Zellen interpretiert. Probe LCl $98 / 5$.

Fig. B: Kollabierte sowie intakte Cyanobakterien-Zellen (Gloeocapsa sp.). Links unten im Bild sind undeutlich Diatomeen-Schalen zu erkennen. Probe LCl 98/5.

Fig. C: Massenhafte Ansammlung von Diatomeen-Schalen, welche eine GloeocapsaKolonie umgeben. Im Zentrum der kokkalen Cyanobakterien befindet sich ein „thrombolite clot““. Probe LCl 98/5.

Fig. D: Ein cyanobakterielles Filament. Ob es sich um eine silifizierte oder karbonatische Imprägnierung unklar. Aufgrund der Größenordnung erfolgt eine Zuordnung zu der Gattung Phormidium sp..Probe LCl 98/7.

Fig. E-F: Charakteristisches büschelartiges Gefüge, welches besonders unter UV-Anregung deutlich wird. E: Hellfeldaufnahme. F: UV-Anregung mit Epifluoreszenz-Filter XF 23. Probe LCl 98/7.

Fig. G-H: Die Mg-Si-Phase mit charakteristischer Färbung und Schrumpfungsrissen der stark hydratisierten Phase. Die SiPhase zeigt eine Fluoreszenz. Diese ist Mischbereich zu den Karbonatklumpen verstärkt. Dichte, mikrokristalline Zentren des Karbonates bleiben ebenso wie rot-braun gefärbte Schlieren innerhalb der amorphen Phase (Pfeile) unter UV-Anregung dunkel. G: Hellfeldaufnahme. H: UVAnregung mit Epifluoreszenz-Filter XF 23. Probe LCl 98/7. 

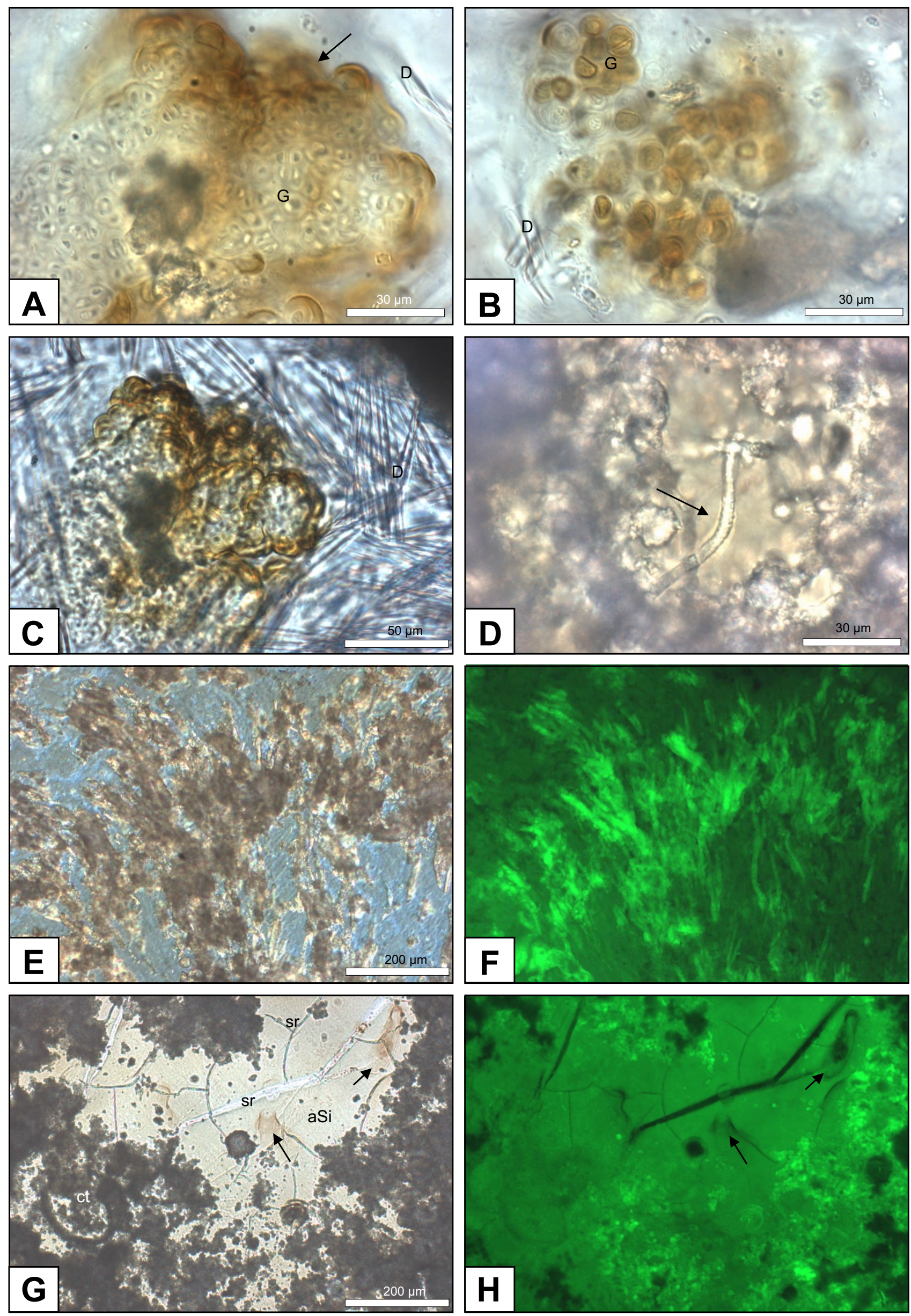


\section{Tafel 9}

\section{Subfossile Thrombolithe, Lake Clifton}

Fig. A: Subrezente Anteile der Bioherme werden von einem unregelmäßig-lappigem Gerüst aus Karbonat-Klumpen (,thrombolite clots“") gebildet. Hohlraumgefüge geben nur grob eine stromatolithische Struktur wider. Probe LCl 98/3.

Fig. B: Der mikrokristalline bis feinfaserige Aragonit enthält radial-büschelförmig angeordnete Scytonema-Filamente. Probe LCl 98/4.

Fig. C: Cyanobakterien-Filamente der Gattung Scytonema sp. im Queranschnitt. Probe LCl $98 / 5$.

Fig. D: In Taschenfüllungen und in lockerem Karbonatsand aus unregelmäßigen Präzipitaten finden sich Gastropoden-Schalen wie Coxella sp. und OstrakodenSchalen (oberer Bildrand). Probe LCl 98/6.

Fig. E: Durch radialstrahlig angeordnete Filamente und gelängte Aragonitpräzipitate erscheint nur grob ein stromatolithisches Gefüge. Die amorphen $\mathrm{Mg}$-Si-Phase ist charakterisiert über die hell-gelbe Färbung und Schrumpfungsrisse. Sie stabilisiert das aragonitische Thrombolith-Gerüst. Probe LCl 98/7.

Fig. F: Diverse cyanobakterielle Filamente sind überliefert, wie kokkale und filamentöse Formen. Nicht einzuordnen ist unter anderem die perlschnurartig aufgereihten Kokken (im Bildausschnitt oben rechts vergrößert). Die Filamentspuren sind durch die Mg-Si-Phase stabilisiert. Hellfeldaufnahme. Probe LCl 98/4.

Fig. G-H: Radialstrahlige und unregelmäßige „thrombolite clots“ Aragonitgerüst, welches von der SiPhase umschlossen wurde. Die amorphe Phase zeigt unter UV-Anregung Fluoreszenz, die dunklen Bereiche markieren Zentren der Karbonatphase und Schrumpfungsrisse. G: Hellfeldaufnahme. H: UV-Anregung, Epifluoreszenz Filter XF 23. Probe LCl 98/7. 
Tafel 9
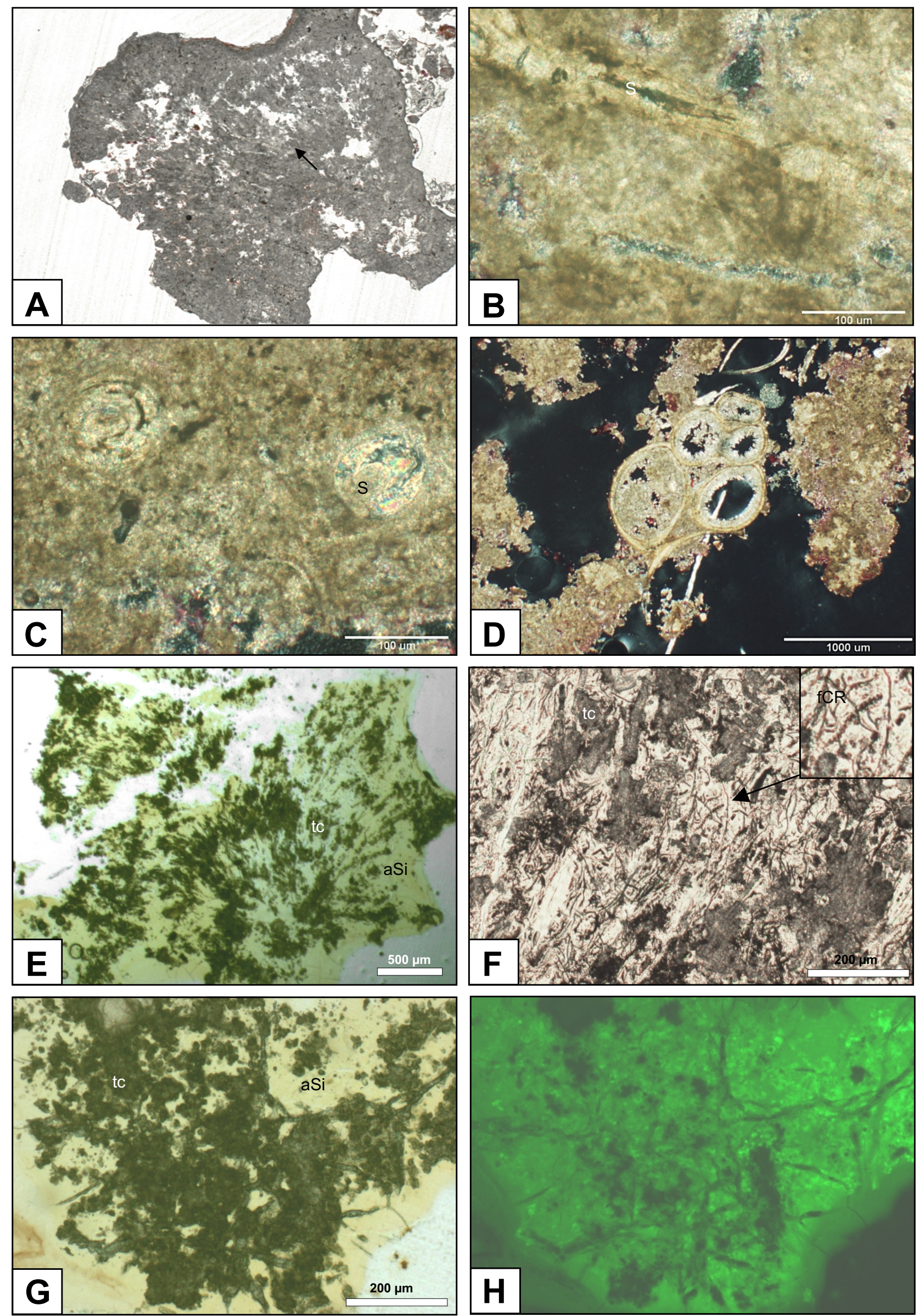


\section{Tafel 10}

\section{Lake Clifton}

Fig. A: Zementierte Cyanobakterien-Filamentspur, Phormidium-Morphotyp. Gekreuzte Polarisatoren. Probe LCl 02/6.

Fig. B: In der amorphen $\mathrm{Mg}-\mathrm{Si}$-Phase sind diverse Filamentspuren, oder wie hier Relikte filamentöser Cyanobakterien eingeschlossen. Hellfeldaufnahme. Probe LCl 02/6.

Fig. C: Ein unkalzifiziertes Cyanobakterien-Trichome mit dicker Scheide, vermutlich Scytonema-Morphotyp. Hellfeldaufnahme. Probe LCL 02/ 6.

Fig. D: Diverse Diatomeen am Rand der Karbonatoberfläche. Im Zentrum sichtbar ist eine zu den Chlorophyta zählende einzellige Grünalge (? Chlamydomonas).

Hellfeldaufnahme. Probe LCl 02/9.

Fig. E-F: Die röntgenamorphe Phase bettet die „thrombolite clots“ ein. Auffallend sind die diffusen Übergänge von der Karbonat- zur SiPhase. Fig. E: Hellfeldaufnahme. Fig. F: gekreuzten Polarisatoren. Probe LCl 02/6.

Fig. G-H: Nicht näher bestimmbare Cyanobakterien und davon unabhängig lokalisierte Karbonatpräzipitate. Fig. G: Hellfeldaufnahme. Fig. H: polarisiertes licht. Probe LCl $02 / 9$. 


\section{Tafel 10}
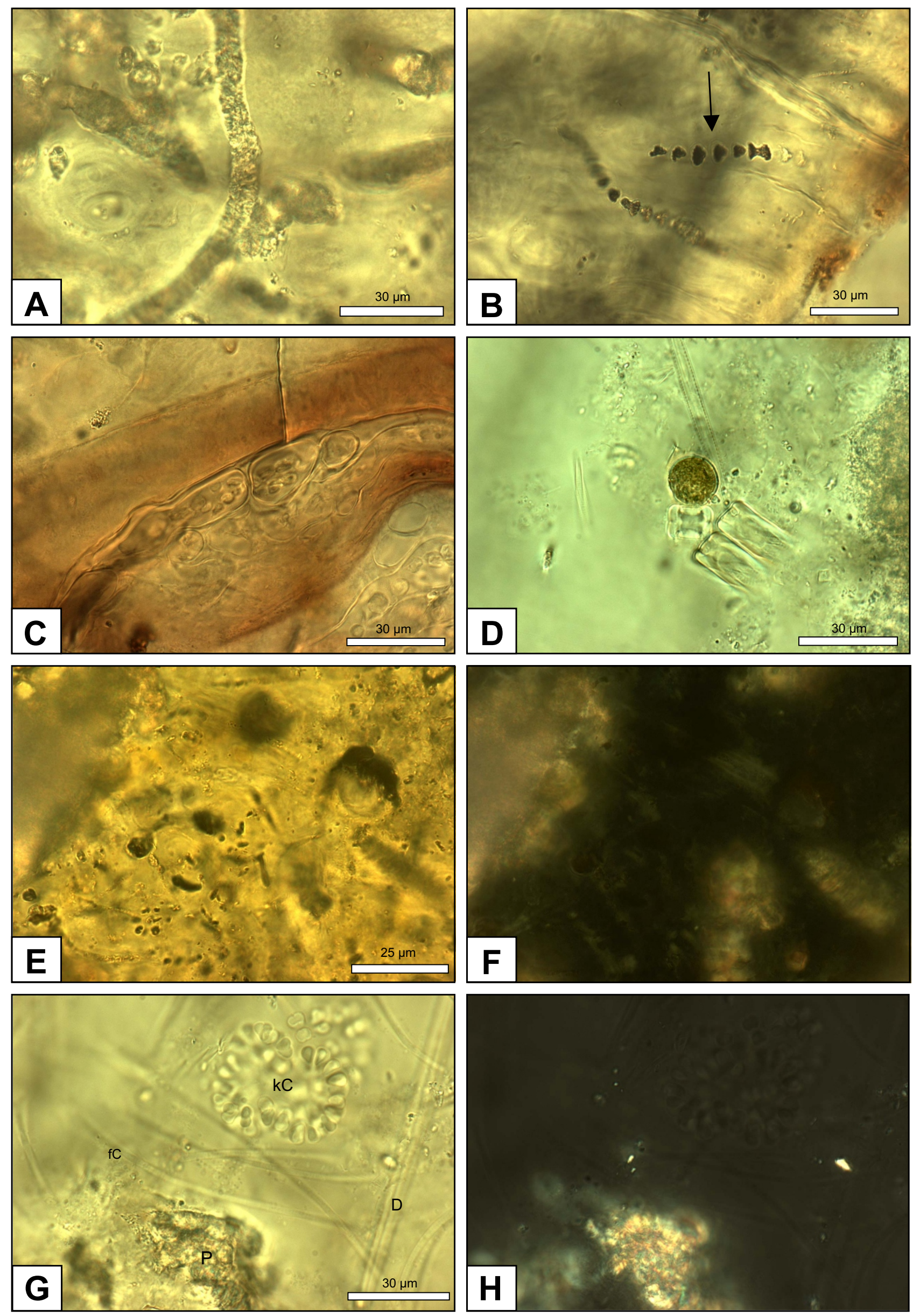


\section{Tafel 11}

\section{Subfossile Mikrobialithe, Lake Clifton und Lake Preston}

Fig. A: Mariner Karbonatsand mit Mollusken-Bruchstücken, Foraminiferen und Rotalgenfragmenten. Hellfeldaufnahme. LPr 98/10.

Fig. B: Mikrobialithische Kruste, die aus feinfaserigen bis mikrokristallinem Aragonit besteht und stellenweise horizontal verlaufende Hohlräume aufweist. Hellfeldaufnahme. LPr 98/10.

Fig. C: Caliche-Kruste, durch einen Wechsel zwischen schlierig laminiertem mikrokristallinem Karbonat und quarzreichen Lagen entsteht makroskopisch ein stromatolithisches Gefüge. Hellfeldaufnahme. LPr 98/10.

Fig. D: Unregelmäßiger Aragonit mit „Organomikrit“-ausgekleideten Hohlräumen (Bildoberkante). Hellfeldaufnahme. LPr 98/10.

Fig. E: Rezent trockengefallener Karbonatschlamm, welcher aus ungleichförmigen mikrokristallinen Arago nit-Klumpen besteht, die in einem organikreichen Mucus eingebettet sind. Hellfeldaufnahme. LPr 02/5.

Fig. F: Erekt bis radial-büschelförmig angeordnete Filamentspuren des Cyanobakteriums Phormidium sp.. Hellfeldaufnahme. LPr 98/10.

Fig. G: Vereinzelt auftretende Characeen-Oogonie in Hellfeldaufnahme mit Taschenfüllung und thrombolithischem Karbonat. Hellfeldaufnahme. Probe LCl $98 / 2$.

Fig. H: Vereinzelte Characeen-Oogonie. Gekreuzte Polarisatoren. Probe LCl 98/1. 

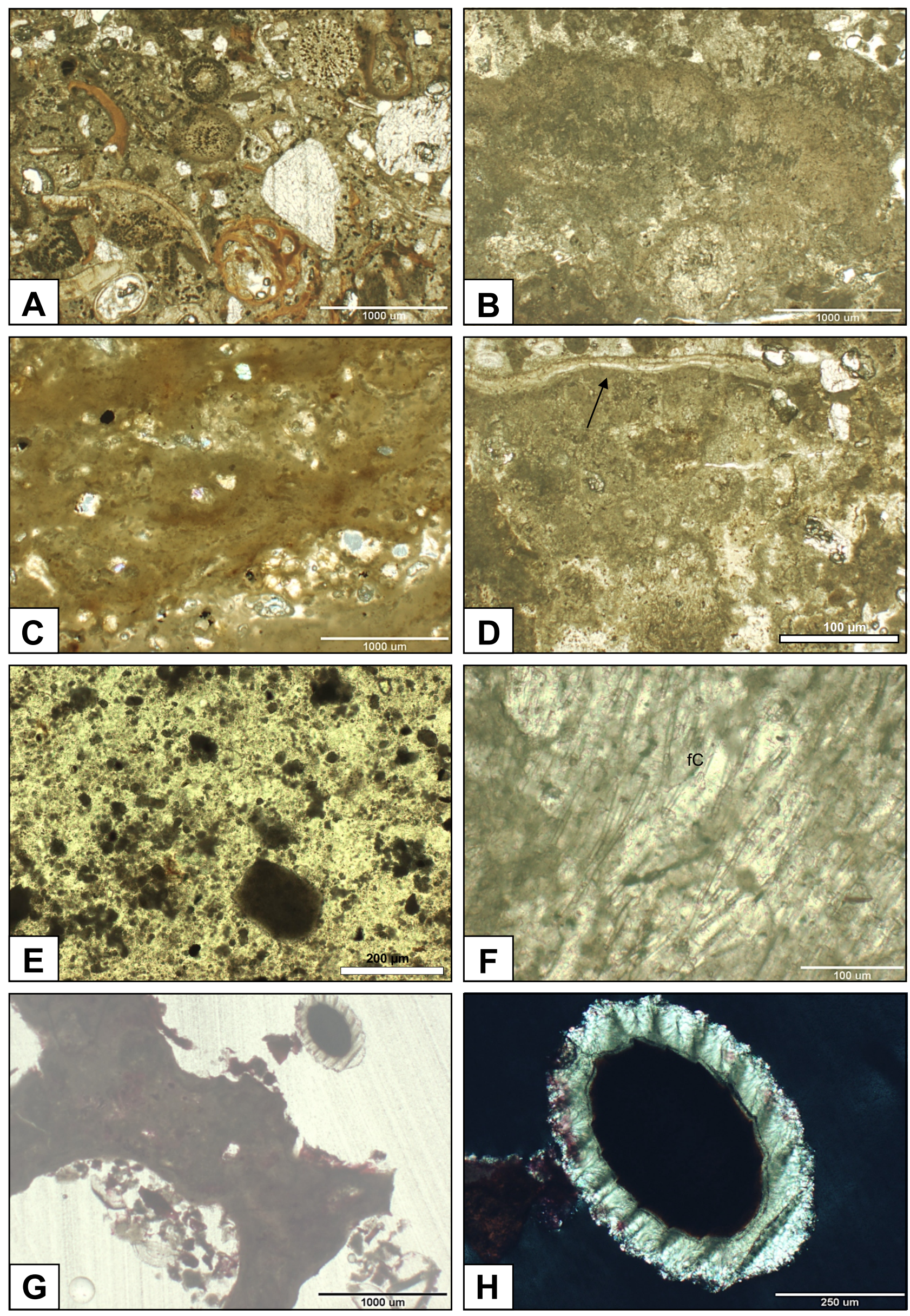


\section{Tafel 12}

\section{Lake Walyungup}

Fig. A: Geländeaufnahme fossiler Mikrobialithe auf der Überschwemmungebene.

Fig. B: Geländeaufnahme, E-Ufer des mittleren Sees, Blick Richtung S. Der Uferbereich ist geprägt von Karbonat-Geröllen und Schottern, vormals thrombolithischer Bioherme und Karbonatkrusten. Knollige Bioherme (fig. A) liegen unregelmäßig, aber gehäuft östlich der Zone der Seegräser.

Fig. C: Rezente Karbonatkrusten mit porösem Gefüge wachsen direkt an der Wasserlinie und werden von Lagen angeschwemmter Algen überdeckt.

Fig. D: Die weißen Karbonatkrusten zeigen schwarze, kristalline Aufwüchse.

Fig. E: Stromatolithische, rot-braune Krusten der fossilen Mikrobialithe. Probe LWa 02/7.

Fig. F: Rezenter Biofilm ist dominiert von filamentösen (fC1 Phormidium sp.; fC2 Scytonema sp. ?) und kokkalen (kC) Cyanobakterien. Hellfeldaufnahme. Probe LWa $02 / 9$.

Fig. G: Radialstrahlig gesproßter Zement (KP) ist unabhängig von dem cyanobakterielldominierten Biofilm bestehend aus filamentösen Cyanobakterien (fC2) lokalisiert. Hellfeldaufnahme. Probe LWa 02/9.

Fig. H: Unkalzifiziertes Filament, Morphotyp Scytonema. Hellfeldaufnahme. Probe LWa $02 / 9$. 

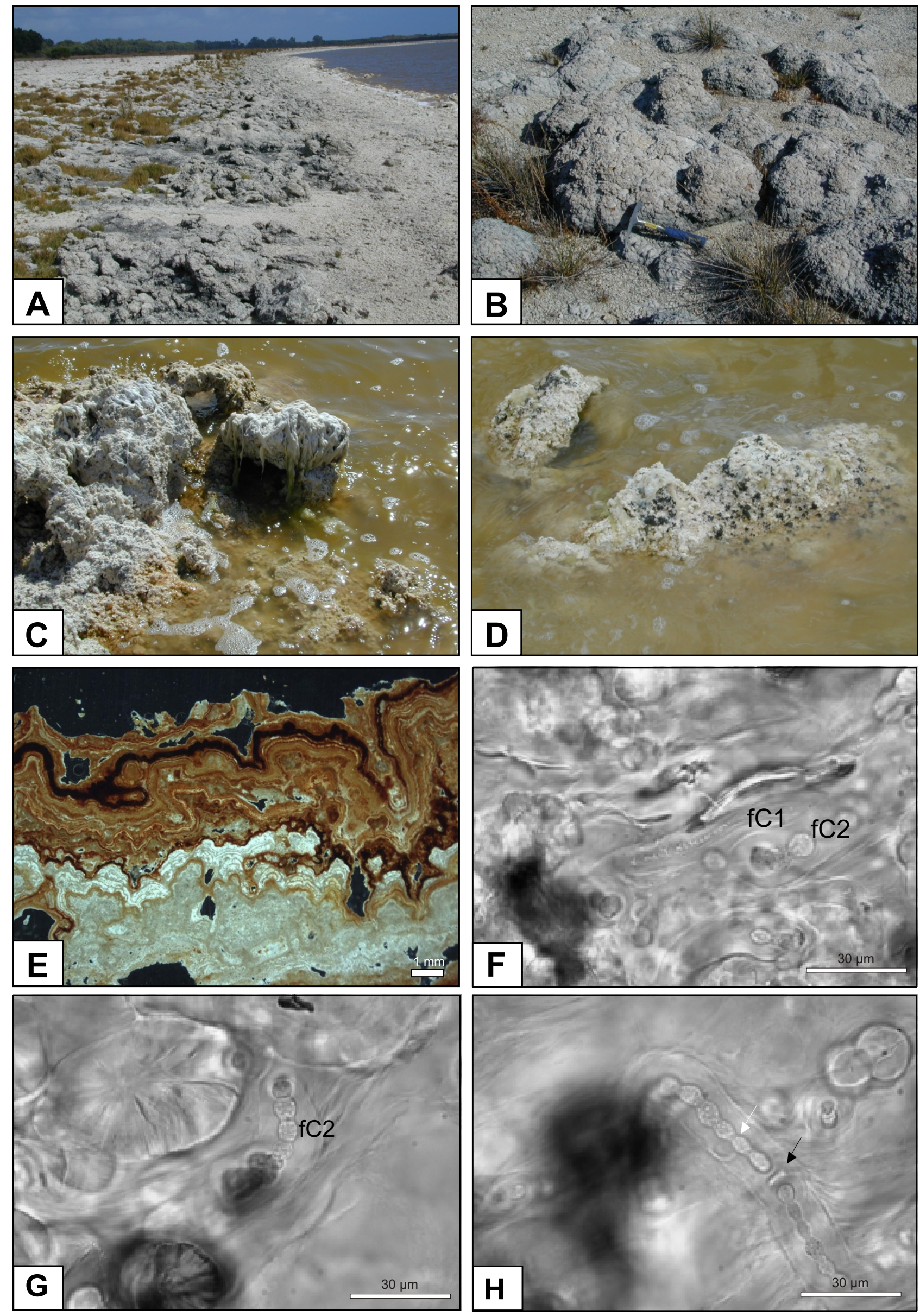


\section{Tafel 13}

\section{Mikrobialithe, Lake Walyungup}

Fig. A-B: Unregelmäßige, mikrokristalline Karbonat-Klumpen bauen ein thrombolithisches Gerüst auf, welches mit Cyanobakterien-Filamenten durchsetzt ist. Die Filamente werden der Gattung Scytonema zu geordnet. Fig. A: Hellfeldaufnahme. Fig. B: Gekreuzte Polarisatoren. LWa 02/9.

Fig. C-F: Hydromagnesit-Gerüst mit erekt bis büschelförmig angeordneten

Cyanobakterien-Filamente. Unter UV-Anregung weisen die Filamente keine Fluoreszenz auf. Nur die Basale Hydromagnesit-Zone weist sowohl unter Filter XF 37 als auch unter Filter Zeiss 01 eine starke Fluoreszenz auf. Fig. C:

Hellfeldaufnahme. Fig. D: Polarisiertes Licht. Fig. E: UV-Anregung, Epifluoreszenz-Filter XF 37. Fig. F: UV-Anregung, Epifluoreszenz-Filter Zeiss 01. Probe LWa 02/9.

Fig. G-H: Ungleichförmiger Mikrit mit aufgewachsenem Pallisaden-Zement.

Ausschließlich die dicht mikritische Zone unterhalb der Zements weist eine starke Fluoreszenz auf. Fig. G: Hellfeldaufnahme. Fig. H: UV-Anregung (Filter Zeiss 01). Probe LWa 02/2. 

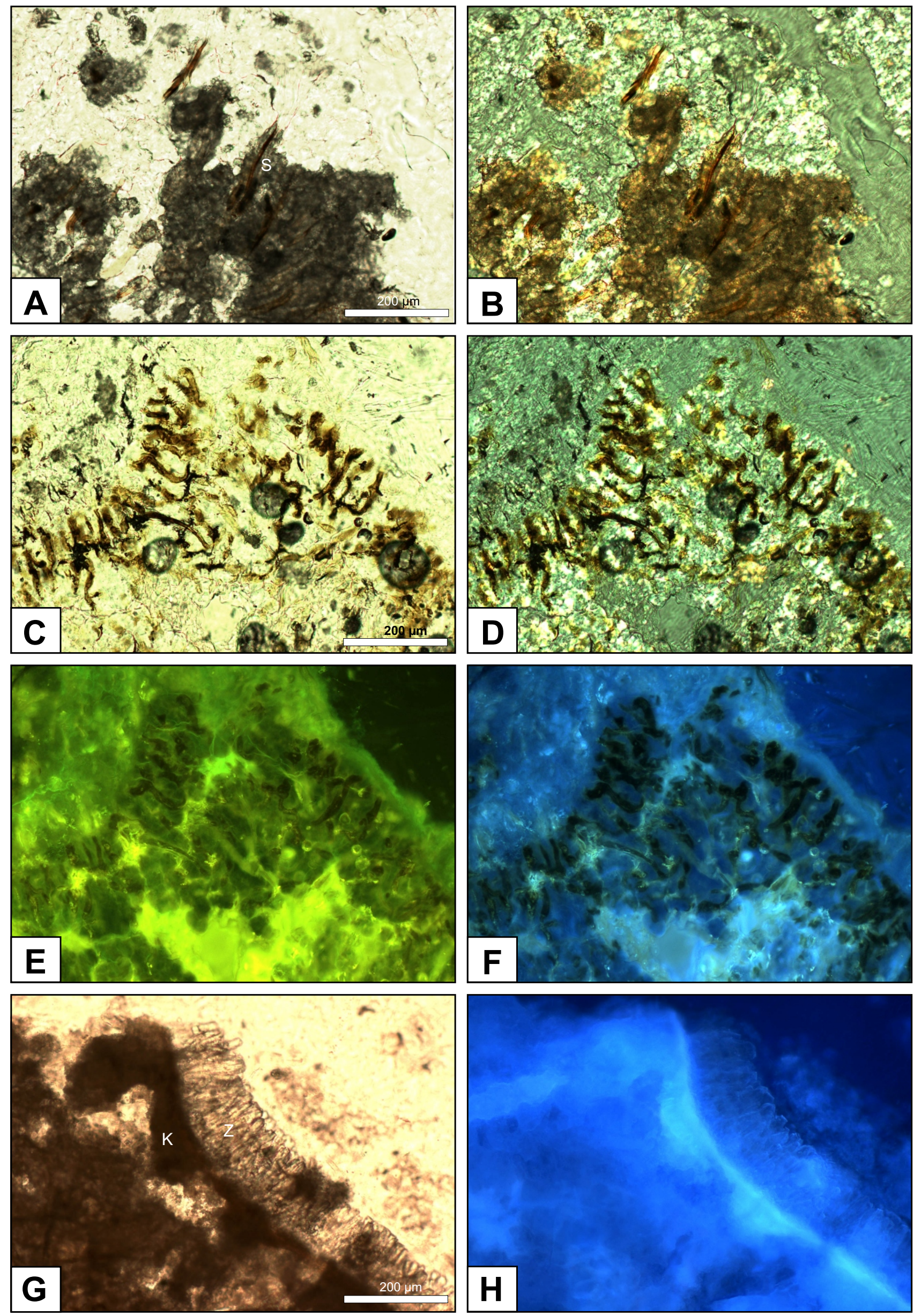


\section{Tafel 14}

\section{Shark Bay}

Fig. A: Geländeaufnahme der fossilen Stromatolithe an Hamelin Pool, Interlitoral.

Fig. B: Die fossilen, agglutinierten Stromatolithe weisen seitliche Anwachsungen grünschwarzer Thrombolithe auf. Diese sind allerdings auf subtidale Bereiche der Stromatolithe begrenzt. Die Wassertiefe beträgt 40-50 cm.

Fig. C: Flachere Stromatolithe sind auch komplett mit thrombolithischen Anwachsungen überzogen. Die Wassertiefe beträgt wenige $\mathrm{cm}$.

Fig. D: Klumpiges Karbonat wird von einem Biofilm umgeben, welcher aus Entophysalis $(\mathrm{kC})$ und filamentösen Cyanobakterien (fC) aufgebaut wird. Hellfeldaufnahme. Probe SB 98/7.

Fig. E-F: Entophysalis und Scytonema-ähnlicher Morphotyp. Die Cyanobakterien sind unkalzifiziert, frisches Präzipitat ist krümelig bis klumpig. Fig. E: DIC. Fig. F: Gekreuzte Polarisatoren. Probe SB 98/7. 
Tafel 14
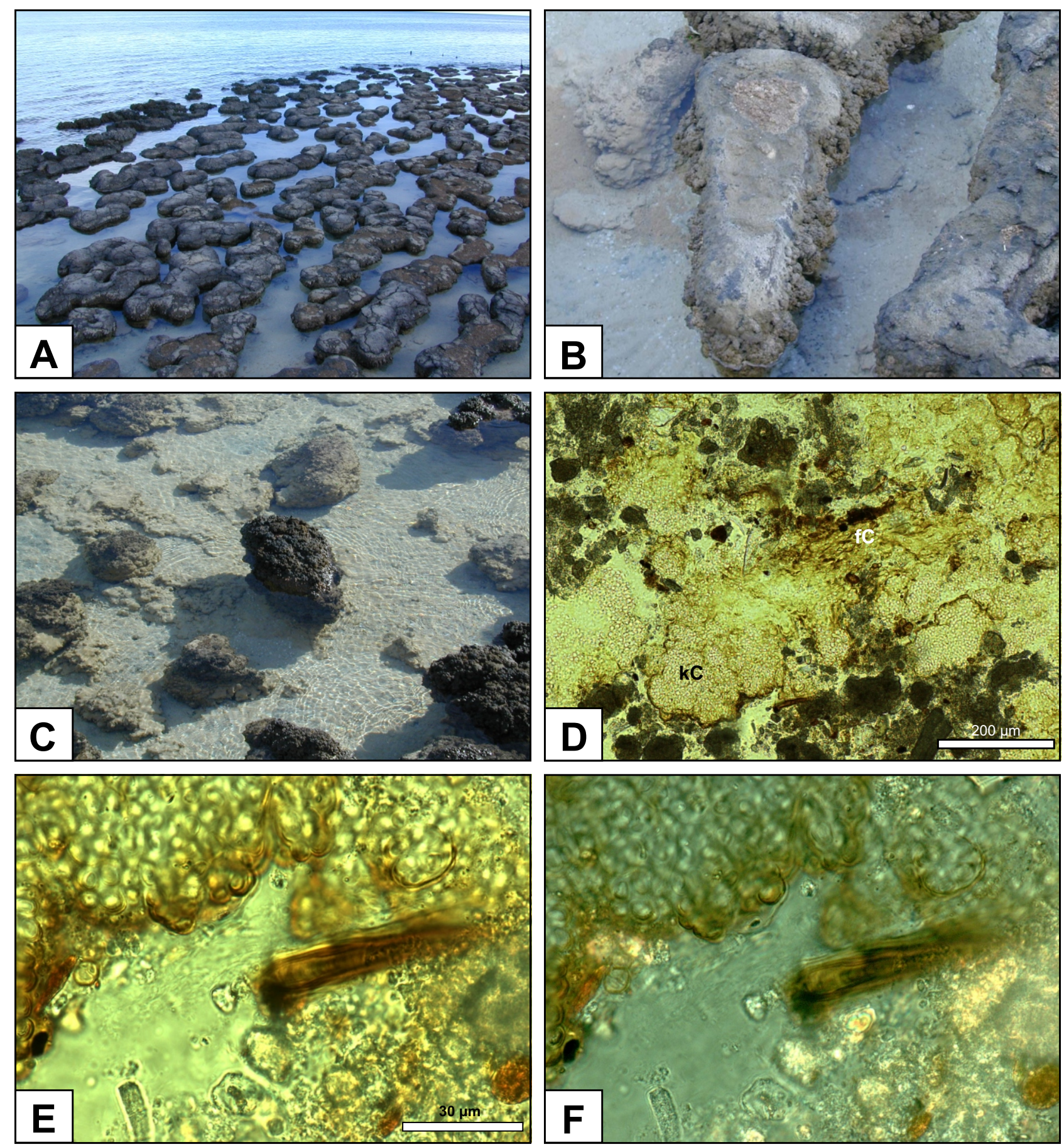


\section{Tafel 15}

\section{Rezente Biofilme, Shark Bay}

Fig. A: Dicker Entophysalis-Biofilm der am äußeren Bereich von eine dünnen, unregelmäßigen Karbonatsaum umgeben ist. Hellfeldaufnahme. Siehe auch Fig. EF. SB 98/7.

Fig. B-D: Entophysalis-Zellen mit angrenzendem Karbonatpräzipitat. Unter UV-Anregung weisen die Zellen im Zentrum wie auch das Karbonat und EPS eine Fluoreszenz auf. Fig. B: Hellfeldaufnahme. Fig. C: DIC + gekreuzte Polaristoren. Fig. D: UVAnregung (Filter XF 23). SB 02/1.

Fig. E-F: Intakte Entophysalis-Kolonien, an Top sich ein Karbonatsaum gebildet hat. Bildausschnitt ist identisch mit dem markierten Bereich in Fig. A. Fig. E: Hellfeldaufnahme. Fig. F: Gekreuzte Polarisatoren. SB 98/7.

Fig. G-H: Rezenter Entophysalis-dominierter Biofilm. Exopolysaccharid-reiche Bereiche sind Karbonatfrei. Unter UV-Anregung zeigen die Entophysalis-Zellen eine Fluoreszenz. Fig. G: DIC. Fig. H: UV-Anregung. SB 98/7. 
Tafel 15
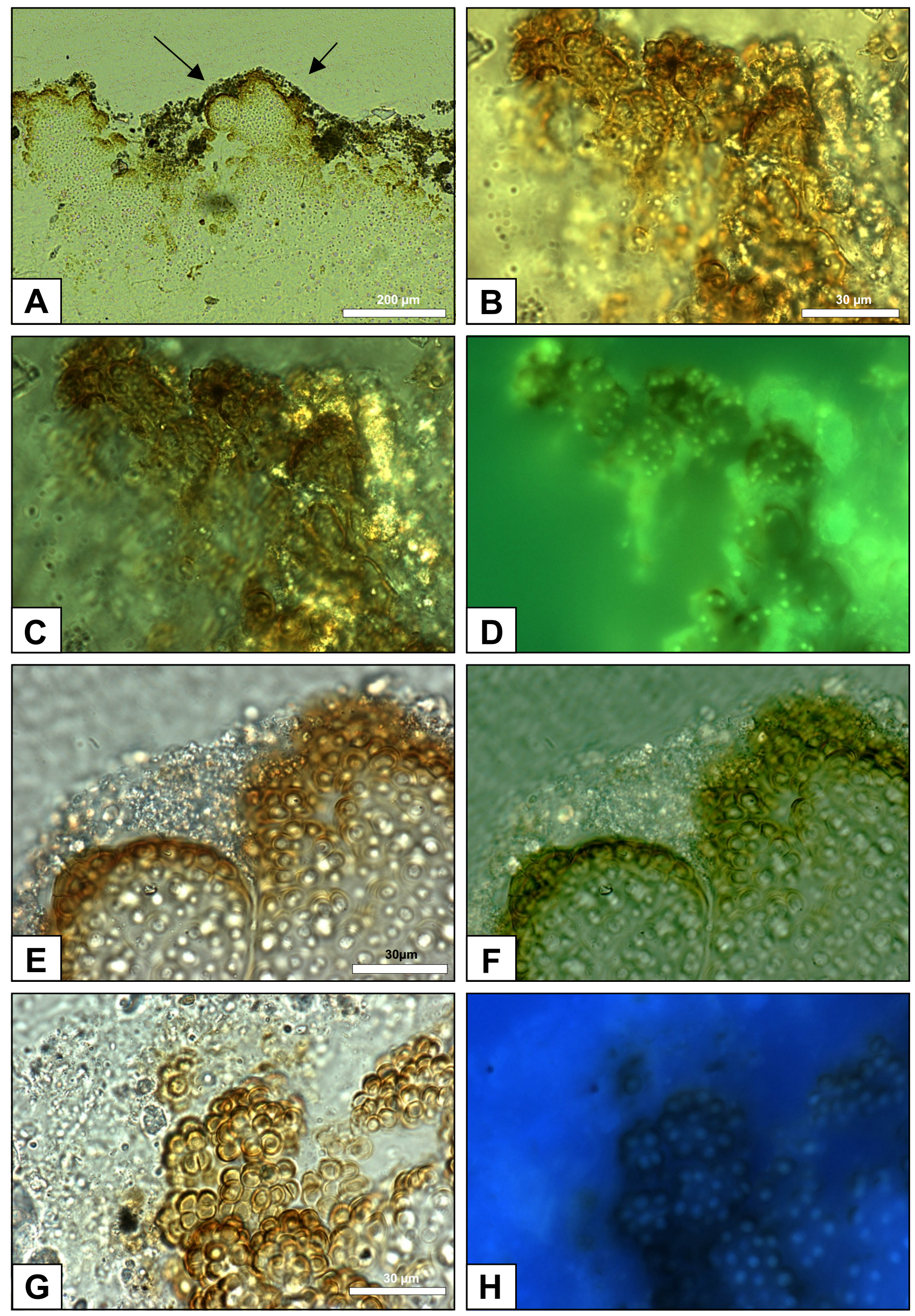


\section{Tafel 16}

\section{Subfossile Stromatolithe, Shark Bay}

Fig. A: Mikrit-Lamelle mit lagenparallelen Hohlräumen. Hellfeldaufnahme. SB 98/18.

Fig. B: Mikrokristallin zementierte Karbonatsandlagen im Wechsel mit wenig zementiertem marinem Karbonatsand. Hellfeldaufnahme. Probe SB 98/1.

Fig. C: Bildausschnitt der mikritisch zementierten Lamelle in Fig. B. Die Komponenten sind mikritisiert und weisen mikrokristalline Karbonatsäume auf. Endolithen sind nicht erkennen. Probe SB 98/2.

Fig. D: Marine Komponenten wie Gastropoden-Schalen, Ooide und Molluskenbruch sind innerhalb des mikrokristallinen, ungleichförmigen Karbonat der knolligen Anwachsungen an den subfossilen Stromatolithen eingebettet. Hellfeldaufnahme.

Fig. E: Die Oberfläche einer knolligen Anwachsung weist unregelmäßige Karbonatklumpen auf, die von einem meteorischen Zement überlagert werden. Hellfeldaufnahme.

Fig. F: Die Knolligen Anwachsungen weisen über fein laminierte Farbwechsel und aneinandergereihte, lagenparallele Hohlraumgefüge ein stromatolithisches Gefüge auf. Hellfeldaufnahme. Probe SB 98/29.

Fig. G: Oberflächen sind großflächig mit Endolithen durchsetzt. Hellfeldaufnahme. Probe SB 98/4.

Fig. H: Fossiler, agglutinierter Stromatolith im Querschnitt mit deutlicher Lamination durch lagenparallele Hohlräume. Probe SB 98/13. 

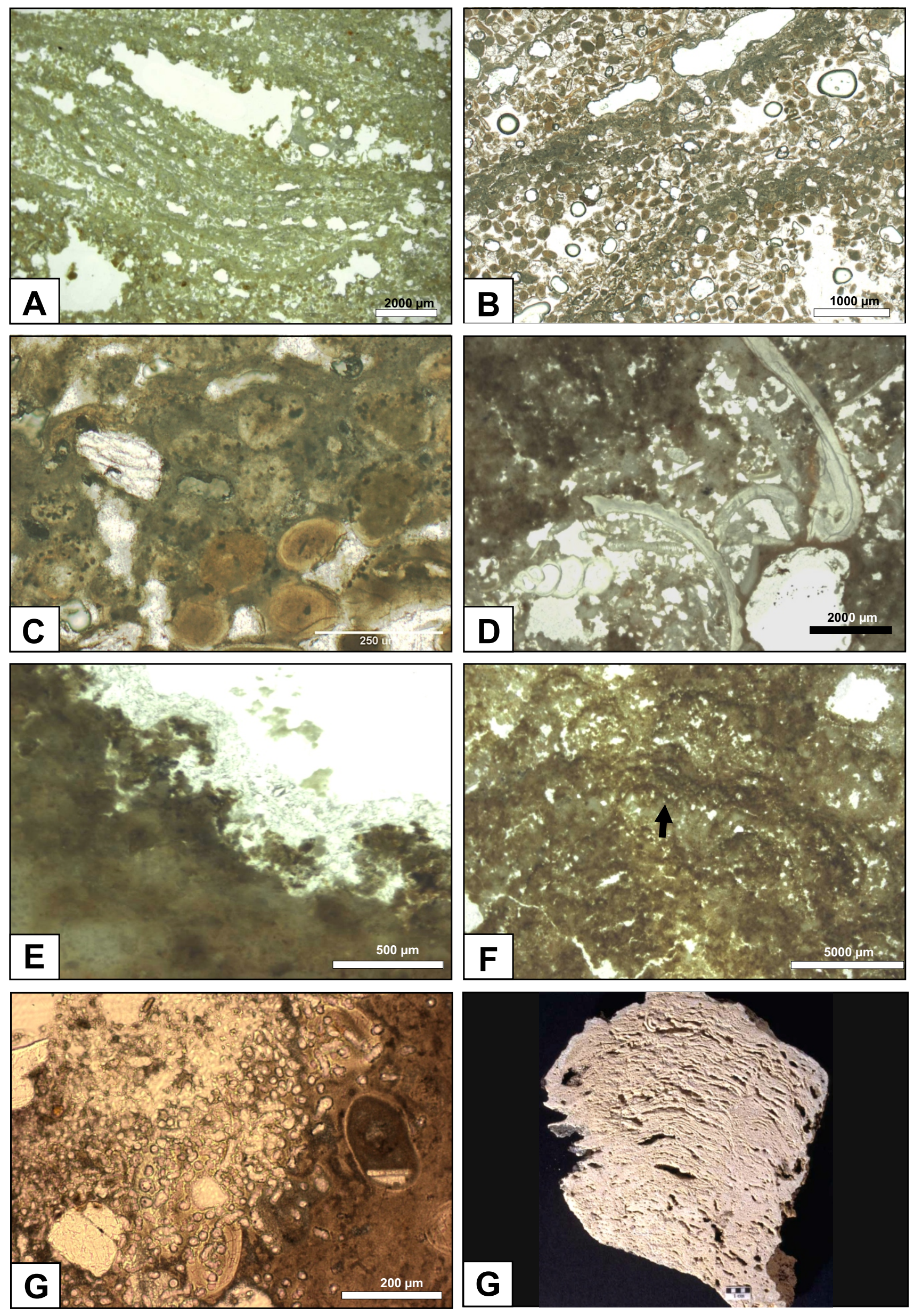


\section{Tafel 17}

\section{Mikrobialithe, Lake Preston und Lake Pollard}

Fig. A: Korngestützte, nicht orientierte Molluskenschalen, schlecht zementierte Quarz- und Karbonatsande und ,thrombolite clots““. Hellfeldaufnahme. Probe LPr 98/9.

Fig. B: Endolithisch überprägte Molluskenschale. Hellfeldaufnahme. Probe LPr 98/9.

Fig. C: Gut bis schlecht gerundete Peloide und Quarzsande sind in lockerer Zementation an Schalenfragment gebunden. Die Molluskenschale zeigt einen mikritischen Saum, der vermutlich auf endolithische Tätigkeit zurück geht. Weitere endolithische Aktivität vollzieht sich von außen in das Schaleninnere hinein. Hellfelsaufnahme. Probe LPr 98/9.

Fig. D: Partikelreicher Aragonit ist mit horizontalen, sandgefüllten Hohlräumen durchzogen, wodurch eine stellenweise ein stromatolithisches Gefüge entsteht. Hellfeldaufnahme. Probe LPr 98/9.

Fig. E: Rotalgen und Foraminiferen sind im Karbonatsand eingebettet. Probe LPr 98/10.

Fig. F: Gleichförmiger Karbonatsand. Probe LPr 98/12.

Fig. G: Der mikrokristalline Aragonit wird von unregelmäßigen Karbonatklumpen aufgebaut. Erkennbar sind darin eingebettete Filamentspuren von Scytonema sp.. Probe LPo 98/4.

Fig. H: Meteorisch alterierter Thrombolith, bestehend aus peloidreichen, schlechtgerundeten Karbonatsanden in Taschenfüllungen und unregelmäßigen „thrombolite clots“(inhomogener, mikrokristalliner Aragonit). Hellfeldaufnahme. Probe LPo 98/4. 

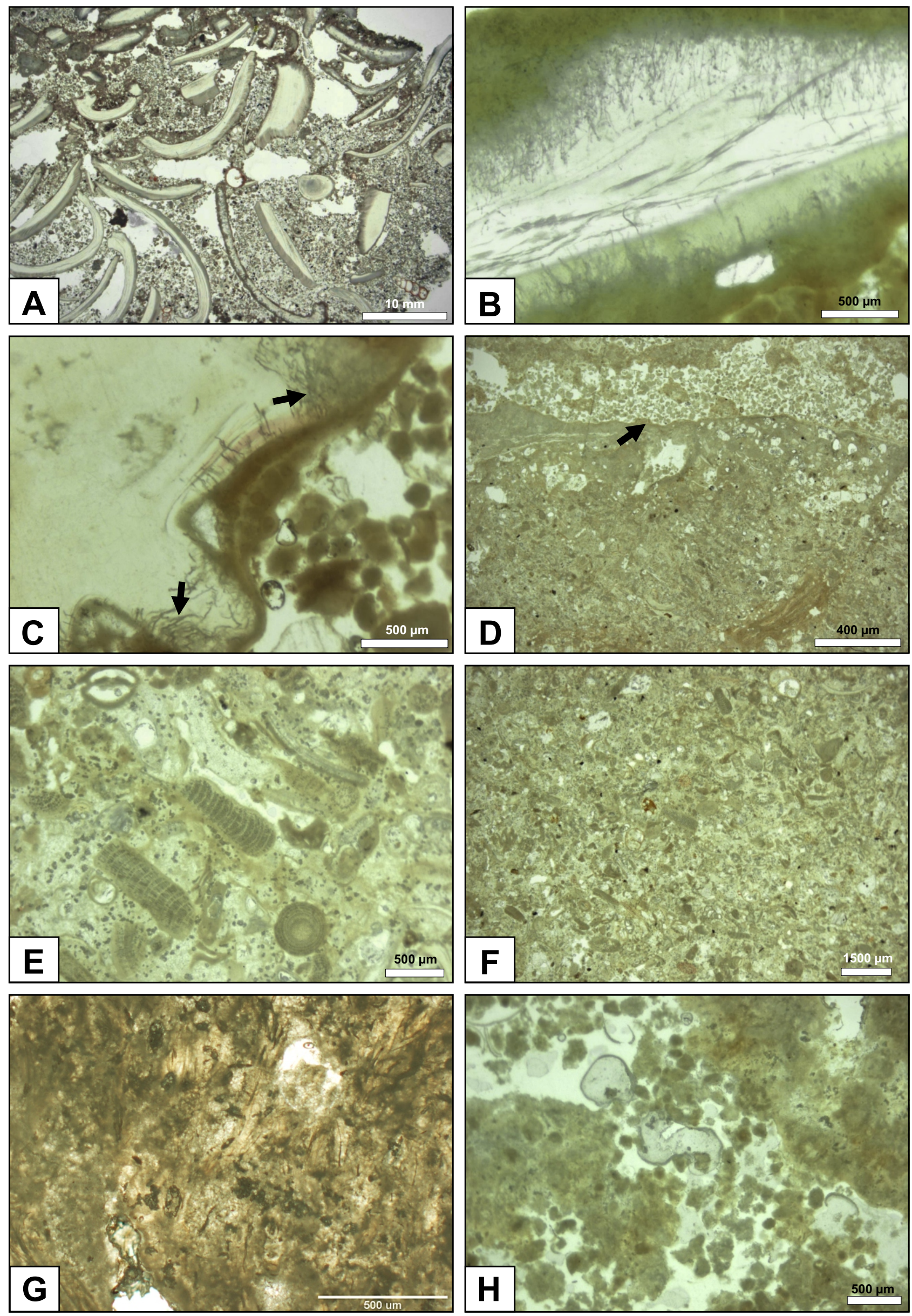


\section{Tafel 18}

\section{Sedimentkerne, Lake Thetis}

Fig. A: Die für die Isotopen-Analysen untersuchten Spherulithe stammen aus Sedimentkernen, die von GREY et al. (1990) vom Seebeckensediment gezogen wurden. Eigene, ca. $50 \mathrm{~cm}$ lange Sedimentkerne beinhalten keine Spherulithe. Die Bezeichnung LT 1bis LT 3 mit zusätzlicher Markierung der Probenentnahme kennzeichnet die Probenbezeichnung der Isotopenproben.

Fig. B: Spherulithe aus dem Sedimentkern, Probe LT 2-4

Fig. C: Spherulith und Karbonataggregat aus dem Sedimentkern, Probe LT 3-1. 
Tafel 18

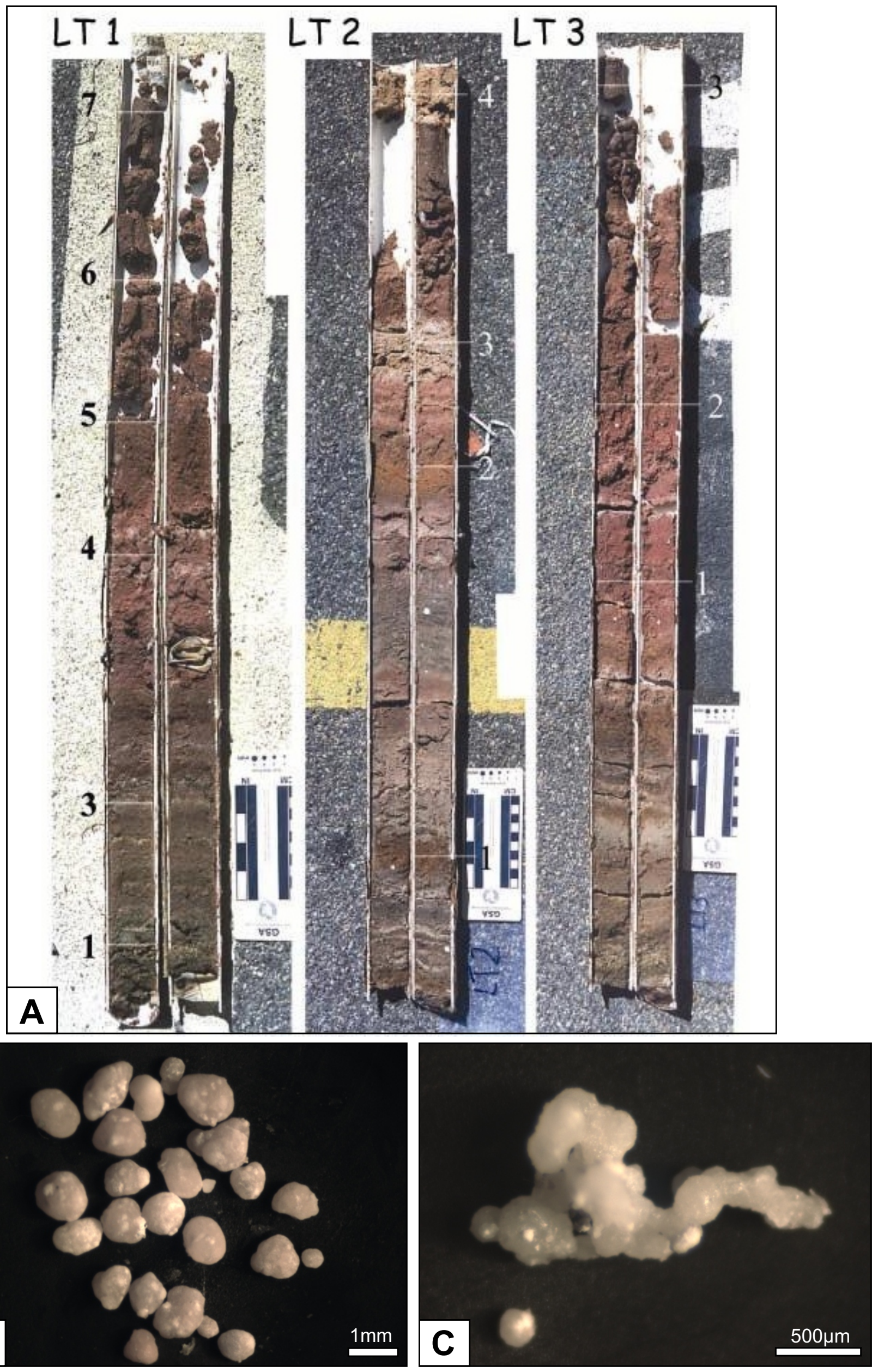


Anhang 1: Probenliste 1998, Regenzeit

\begin{tabular}{|c|c|c|c|c|}
\hline Proben-Nr. & Lokation & $\begin{array}{l}\text { Wasser- } \\
\text { tiefe }\end{array}$ & Beschreibung & Datum \\
\hline LPr98/1 & Lake Preston, NE-Ufer S-Arm & - & $\begin{array}{l}\text { Rötlich, gummiartige organische Matte vom Rand des } \\
\text { Zuflusses des N-Arm; Top leicht mineralisiert }\end{array}$ & $09 / 08 / 98$ \\
\hline LPr98/2 & Lake Preston, NE-Ufer S-Arm & - & Dunkel-grün Matte, feuchte Sandfläche & $09 / 08 / 98$ \\
\hline LPr98/3 & Lake Preston, NE-Ufer S-Arm & - & $\begin{array}{l}\text { Dunkel-grün bis braun pustulare Matte von der } \\
\text { Sandfläche, trocken; leicht ausgeblichen und } \\
\text { mineralisiert }\end{array}$ & $09 / 08 / 98$ \\
\hline LPr98/4 & Lake Preston, NE-Ufer S-Arm & - & $\begin{array}{l}\text { Trockene, grüne gummiartige Matte mit } \\
\text { Schrumpfungsrissen und weißem, plattigem Karbonat }\end{array}$ & $09 / 08 / 98$ \\
\hline LPr98/5 & Lake Preston, NE-Ufer S-Arm & - & Dunkel-pinke Matte, vom Ufer & $09 / 08 / 98$ \\
\hline LPr98/6 & Lake Preston, SE-Ufer N-Arm & - & Schwarz-grüne Matte & $12 / 08 / 98$ \\
\hline LPr98/7 & Lake Preston, SE-Ufer N-Arm & - & Karbonat-Kruste von flacher Ebene & $12 / 08 / 98$ \\
\hline LPr98/8 & Lake Preston, SE-Ufer N-Arm & - & Schnecken-Akkumulat & $12 / 08 / 98$ \\
\hline LC98/1 & $\begin{array}{l}\text { Lake Clifton, E-Ufer (ca. 1km N of } \\
\text { walkway Mt. John Road) }\end{array}$ & - & $\begin{array}{l}\text { "versicolored mat" auf weichem Sediment, } \\
\text { Überschwemmungsebene }\end{array}$ & $11 / 08 / 98$ \\
\hline LC98/2 & $\begin{array}{l}\text { Lake Clifton, E-Ufer (ca. 1km N of } \\
\text { walkway Mt. John Road) }\end{array}$ & $5-10 \mathrm{~cm}$ & $\begin{array}{l}\text { Schwarz-grüner Biofilm, Seite flach konzentrischer } \\
\text { Bioherm (seasonal exponiert) }\end{array}$ & $11 / 08 / 98$ \\
\hline LC98/3 & $\begin{array}{l}\text { Lake Clifton, E-Ufer (ca. 1km N of } \\
\text { walkway Mt. John Road) }\end{array}$ & $30-40 \mathrm{~cm}$ & Gelber Biofilm vom Top (spherical bioherm) & $11 / 08 / 98$ \\
\hline LC98/4 & $\begin{array}{l}\text { Lake Clifton, E-Ufer ( } 25 \mathrm{~m} \mathrm{~N} \text { of } \\
\text { walkway) }\end{array}$ & $50 \mathrm{~cm}$ & $\begin{array}{l}\text { Top von kegelförmigen Bioherm (vent-like opening); } \\
\text { gelber Biofilm, grün darunter, auf hochporösem } \\
\text { thrombolithischem Karbonat }\end{array}$ & $11 / 08 / 98$ \\
\hline LC98/5 & $\begin{array}{l}\text { Lake Clifton, E-Ufer (ca. 1km N of } \\
\text { walkway Mt. John Road) }\end{array}$ & $20-30 \mathrm{~cm}$ & $\begin{array}{l}\text { "versicolored microbial mat" auf weichem Sediment } \\
\text { zwischen den Biohermen }\end{array}$ & $11 / 08 / 98$ \\
\hline LC98/6 & $\begin{array}{l}\text { Lake Clifton, E-Ufer (ca. 1km N of } \\
\text { walkway Mt. John Road) }\end{array}$ & $35-40 \mathrm{~cm}$ & Lockerer Karbonatsand zwischen den Biohermen & $11 / 08 / 98$ \\
\hline LC98/7 & $\begin{array}{l}\text { Lake Clifton, E-Ufer (ca. } 1 \mathrm{~km} \mathrm{~N} \text { of } \\
\text { walkway Mt. John Road) }\end{array}$ & 0 & $\begin{array}{l}\text { Kante eines flachen, konzentrischen Bioherms } \\
\text { (brownish-reddish voids) }\end{array}$ & $11 / 08 / 98$ \\
\hline LTh98/1 & Lake Thetis, S-Ufer & $2 \mathrm{~m}$ & $\begin{array}{l}\text { Violette Bakteriensuspension, Seeboden, }(10 \mathrm{~cm} \\
\text { dicke Lage auf weichem Sediment }\end{array}$ & $21 / 08 / 98$ \\
\hline LTh98/2 & Lake Thetis, S-Ufer & $1.8 \mathrm{~m}$ & $\begin{array}{l}\text { pustularer Biofilm (gelb-braune Oberfläche, grün } \\
\text { darunter) auf Thrombolith }\end{array}$ & $21 / 08 / 98$ \\
\hline LTh98/3 & Lake Thetis, S-Ufer & $1.8 \mathrm{~m}$ & Thrombolite nodule; analog zu LTh98/2 & $21 / 08 / 98$ \\
\hline LTh98/4 & Lake Thetis, S-Ufer & $0.3 \mathrm{~m}$ & Schwarz-grüne Oberfläche vom Bioherm-Rand & $21 / 08 / 98$ \\
\hline LTh98/5 & Lake Thetis, S-Ufer & $0.5 \mathrm{~m}$ & $\begin{array}{l}\text { Schwarz-grüne, knotige Oberfläche von Bioherm- } \\
\text { Seite (partly coarse-layered fabric) }\end{array}$ & $21 / 08 / 98$ \\
\hline LTh98/6 & Lake Thetis, S-Ufer & - & $\begin{array}{l}\text { Schwarz-grüne pustular Matte von exponierter Ebene } \\
2 \mathrm{~m} \text { von der Uferlinie (Regenwassereinfluss). }\end{array}$ & $21 / 08 / 98$ \\
\hline LTh98/7 & Lake Thetis, S-Ufer & $10 \mathrm{~cm}$ & $\begin{array}{l}\text { Top von erodierten, kleinen Bioherm; schwach- } \\
\text { gelbliche Biofilme auf Top und Seite; dunkelgrüne in } \\
\text { Rissen; }\end{array}$ & $21 / 08 / 98$ \\
\hline LTh98/8 & Lake Thetis, S-Ufer & $10 \mathrm{~cm}$ & $\begin{array}{l}\text { massive, weiße Kruste vom Bioherm (flooded, } \\
\text { inactive) }\end{array}$ & $21 / 08 / 98$ \\
\hline LTh98/9 & $\begin{array}{l}\text { Lake Thetis, S-Ufer, } \\
\text { Überflutungsebene }\end{array}$ & - & Karbonat vom Strandrand (Caliche) & $21 / 08 / 98$ \\
\hline LTh98/10 & Lake Thetis, S-Ufer & $2.5 \mathrm{~m}$ & Sedimentsuspension vom Seeboden & $22 / 08 / 98$ \\
\hline LTh98/11 & Lake Thetis, S-Ufer & $30 \mathrm{~cm}$ & $\begin{array}{l}\text { Dunkelgrüner, weiß-gepunkteter Biofilm vom } \\
\text { Karbonatsaum zwischen den Biohermen }\end{array}$ & $22 / 08 / 98$ \\
\hline LTh98/12 & Lake Thetis, N-Ufer & - & exponierter, "dead thrombolite" (erodiert) & $22 / 08 / 98$ \\
\hline LTh98/13 & Lake Thetis, S-Ufer & $10 \mathrm{~cm}$ & äußere, pustulare Kruste von Bioherm & $22 / 08 / 98$ \\
\hline LTh98/14 & Lake Thetis, S-Ufer & $10 \mathrm{~cm}$ & innere Kruste von Bioherm & $22 / 08 / 98$ \\
\hline LTh98/15 & Lake Thetis, S-Ufer & $30 \mathrm{~cm}$ & $\begin{array}{l}\text { Flache Kruste von Karbonatsaum zwischen den } \\
\text { Biohermen }\end{array}$ & $22 / 08 / 98$ \\
\hline SB98/1 & Shark Bay, Carbla Point & $1 \mathrm{~m}(\mathrm{NW})$ & $\begin{array}{l}\text { Gelb-grüne, leicht pinke, weiche Matte vom Top eines } \\
\text { subtidalen Stromatolith }\end{array}$ & $17 / 08 / 98$ \\
\hline SB98/2 & Shark Bay, Carbla Point & $\begin{array}{l}1.2 \mathrm{~m} \\
(\mathrm{NW})\end{array}$ & $\begin{array}{l}\text { Gelber Biofilm (grüne Unterlage, schwarzer Kern) } \\
\text { vom seitlichen Top subtidaler Stromatolith; mit } \\
\text { angehafteten Algen Dascyladacean (Acetabularia) }\end{array}$ & $17 / 08 / 98$ \\
\hline SB98/3 & Shark Bay, Carbla Point & $\begin{array}{l}10-20 \mathrm{~cm} \\
(\mathrm{NW})\end{array}$ & $\begin{array}{l}\text { Hell bis dunkelgrauer, fleckiger Biofilm (olive-grün } \\
\text { cyanobakterille Kissen; grassgrüne Unterlageweißer } \\
\text { Kern) vom Top sub/intertidal Stromatolith }\end{array}$ & $17 / 08 / 98$ \\
\hline SB98/4 & Shark Bay, Carbla Point & $\begin{array}{l}+/-0 m \\
(\mathrm{NW})\end{array}$ & $\begin{array}{l}\text { Dunkel oliv-grüner Biofilm vom Top intertidaler } \\
\text { Stromatolith }\end{array}$ & $17 / 08 / 98$ \\
\hline SB98/5 & Shark Bay, Carbla Point & $\begin{array}{l}+/-0 \mathrm{~m} \\
(\mathrm{NW})\end{array}$ & $\begin{array}{l}\text { tufted-reticulate, dark-green mat auf lockerem weißen } \\
\text { Karbonatsand (zwischen Stromatoliten und mamillate } \\
\text { Entophysalis mat) }\end{array}$ & $17 / 08 / 98$ \\
\hline SB98/6 & $\begin{array}{l}\text { Shark Bay, Telegraph Station, } \\
\text { Hamelin Pool }\end{array}$ & $\begin{array}{l}+/-0 m \\
(\mathrm{NW})\end{array}$ & red cap an der Wasserkante & $18 / 08 / 98$ \\
\hline SB98/7 & $\begin{array}{l}\text { Shark Bay, Telegraph Station, } \\
\text { Hamelin Pool }\end{array}$ & $\begin{array}{l}+/-0 m \\
(N W)\end{array}$ & $\begin{array}{l}\text { mamillate Entophysalis mat zwischen Stromatolith- } \\
\text { Köpfen und Sediment }\end{array}$ & $18 / 08 / 98$ \\
\hline SB98/8 & $\begin{array}{l}\text { Shark Bay, Telegraph Station, } \\
\text { Hamelin Pool }\end{array}$ & $\begin{array}{l}+10 \mathrm{~cm} \\
\text { (NW) }\end{array}$ & $\begin{array}{l}\text { Dunkel grün verfestigte pustules vom glatten Top des } \\
\text { Stromatoliths }\end{array}$ & $18 / 08 / 98$ \\
\hline SB98/9 & $\begin{array}{l}\text { Shark Bay, Telegraph Station, } \\
\text { Hamelin Pool }\end{array}$ & $1 \mathrm{~m}(\mathrm{NW})$ & $\begin{array}{l}\text { cm-großer subtidaler Stromatolith-Kegel (coarse } \\
\text { laminated, pale core) zwischen lockerem Sand; } \\
\text { äußere Oberfläche mit orangenerm dicken matte mit }\end{array}$ & $18 / 08 / 98$ \\
\hline
\end{tabular}




\begin{tabular}{|c|c|c|c|c|}
\hline & & & $\begin{array}{l}\text { green underlayer; core pale-grey to white; laterale } \\
\text { Seiten mit Acetabularia }\end{array}$ & \\
\hline SB98/10 & $\begin{array}{l}\text { Shark Bay, Telegraph Station, } \\
\text { Hamelin Pool }\end{array}$ & $\begin{array}{l}30 \mathrm{~cm} \\
\text { (low to } \\
\text { high tide) }\end{array}$ & $\begin{array}{l}\text { Schwarz-grüner mamillare Entophysalis Matte } \\
\text { (sedimentreich) von Stromatolith-Basis }\end{array}$ & $18 / 08 / 98$ \\
\hline SB98/11 & $\begin{array}{l}\text { Shark Bay, Telegraph Station, } \\
\text { Hamelin Pool }\end{array}$ & $\begin{array}{l}1 \mathrm{~m}(\mathrm{H}- \\
\mathrm{NW})\end{array}$ & $\begin{array}{l}\text { Top und seitliches Top von subtidalem Stromatolith, } \\
\text { orange-braun, glatte Matte (green patches); wenig } \\
\text { Acetabularia }\end{array}$ & $18 / 08 / 98$ \\
\hline SB98/12 & $\begin{array}{l}\text { Shark Bay, Telegraph Station, } \\
\text { Hamelin Pool }\end{array}$ & $\begin{array}{l}1.2 \mathrm{~m}(\mathrm{H}- \\
\mathrm{NW})\end{array}$ & $\begin{array}{l}\text { Flaches Stromatolith-Top zwischen lockerem Sand; } \\
\text { orange Matte (grüne Unterlage) reich an Sediment- } \\
\text { partikeln, Poren mit orange-braunem Film, Kern } \\
\text { hellgrau bis weiß }\end{array}$ & $18 / 08 / 98$ \\
\hline SB98/13 & $\begin{array}{l}\text { Shark Bay, Telegraph Station, } \\
\text { Hamelin Pool }\end{array}$ & $1 \mathrm{~m}(\mathrm{NW})$ & Gelb-grüne, gefleckte Matte vom Stromatolith-Top & $19 / 08 / 98$ \\
\hline SB98/14 & $\begin{array}{l}\text { Shark Bay, Telegraph Station, } \\
\text { Hamelin Pool }\end{array}$ & $1 \mathrm{~m}(\mathrm{NW})$ & $\begin{array}{l}\text { Orange-gelbe, glatte Matte vom Top eines kleinen } \\
\text { konischen Stromatolithen (coarse laminated) }\end{array}$ & $19 / 08 / 98$ \\
\hline SB98/15 & $\begin{array}{l}\text { Shark Bay, Telegraph Station, } \\
\text { Hamelin Pool }\end{array}$ & $\begin{array}{l}+/-0 \\
\text { (extrem } \\
\text { NW) }\end{array}$ & $\begin{array}{l}\text { semi-lithifiziert oliv-grün, fein-mamillar Rans von } \\
\text { expononiertem Stromatolith }\end{array}$ & $20 / 08 / 98$ \\
\hline
\end{tabular}


Anhang 2: Probenliste 2002, Trockenzeit

\begin{tabular}{|c|c|c|c|c|}
\hline Proben-Nr. & Lokation & $\begin{array}{l}\text { Wasser- } \\
\text { tiefe }\end{array}$ & Beschreibung & Datum \\
\hline LPr02/1/1 & Lake Preston, NE-Ufer S-Arm & - & $\begin{array}{l}\text { Handstück, Thrombolith auf Muscheln und } \\
\text { Basement }\end{array}$ & 06/04/02 \\
\hline LPr02/1/2 & Lake Preston, NE-Ufer S-Arm & - & $\begin{array}{l}\text { Handstück, Thrombolith auf Muscheln und } \\
\text { Basement }\end{array}$ & 06/04/02 \\
\hline LPr02/2 & Lake Preston, NE-Ufer S-Arm & - & Basement mit Thrombolith-Top & $06 / 04 / 02$ \\
\hline LPr02/3 & Lake Preston, NE-Ufer S-Arm & - & Karbonatkuste vom Plateau & $06 / 04 / 02$ \\
\hline LPr02/4 & Lake Preston, NE-Ufer S-Arm & - & $\begin{array}{l}\text { Grau, nodular organisches Material (trockenes } \\
\text { Seebecken), 2-3 cm Durchmesser }\end{array}$ & 06/04/02 \\
\hline LPr02/5 & Lake Preston, NE-Ufer S-Arm & - & $\begin{array}{l}\text { organisches Material, grau, nodular (trockenes } \\
\text { Seebecken), 0,5-1 cm Durchmesser }\end{array}$ & $06 / 04 / 02$ \\
\hline LWa02/1 & Lake Walyungup, E-Ufer, S-Becken & $0-2 \mathrm{~cm}$ & Algen, grün ("hanky-like”), Spritzwasserzone & 07/04/02 \\
\hline $\mathrm{LWa02/2}$ & Lake Walyungup, E-Ufer, S-Becken & $0-2 \mathrm{~cm}$ & Weiße Kruste, unterhalb der Wasserlinie & $07 / 04 / 02$ \\
\hline LWa02/3 & Lake Walyungup, E-Ufer, S-Becken & 0 & Handstück, "white crystalline crust" & $07 / 04 / 02$ \\
\hline $\mathrm{LWa02/4}$ & Lake Walyungup, E-Ufer, S-Becken & 0 & Handstück, "white crystalline crust " & $07 / 04 / 02$ \\
\hline LWa02/5 & Lake Walyungup, E-Ufer, S-Becken & - & Fossiler Stromatolith & $07 / 04 / 02$ \\
\hline $\mathrm{LWa02/6}$ & Lake Walyungup, E-Ufer, S-Becken & - & Fossiler Stromatolith, äußerer Teil & $07 / 04 / 02$ \\
\hline $\mathrm{LWa02/7}$ & Lake Walyungup, E-Ufer, S-Becken & - & Fossiler Stromatolith, innerer Teil & $07 / 04 / 02$ \\
\hline LWa02/8 & Lake Walyungup, E-Ufer, S-Becken & - & Fossiler Stromatolith, äußerer Teil & $07 / 04 / 02$ \\
\hline LWa02/9 & Lake Walyungup, E-Ufer, S-Becken & $+/-0 \mathrm{~cm}$ & $\begin{array}{l}\text { Schwarze, kubische "dots and mats", } \\
\text { interlitoral/splashing zone }\end{array}$ & $07 / 04 / 02$ \\
\hline $\mathrm{LCl02/1}$ & $\begin{array}{l}\text { Lake Clifton, E-Ufer (ca. 1km N of } \\
\text { walkway Mt. John Road), pond }\end{array}$ & $+/-0 \mathrm{~cm}$ & $\begin{array}{l}\text { "versicoloured mat" auf weichem Sediment, } \\
\text { ponds, Überschwemmungsebene }\end{array}$ & $9 / 04 / 02$ \\
\hline $\mathrm{LCl02/2}$ & $\begin{array}{l}\text { Lake Clifton, E-Ufer (ca. 1km N of } \\
\text { walkway Mt. John Road), pond }\end{array}$ & $+/-0 \mathrm{~cm}$ & $\begin{array}{l}\text { Fibröser, weißer Biofilm auf } \\
\text { Überschwemmungsebene, exponiert }\end{array}$ & $9 / 04 / 02$ \\
\hline $\mathrm{LCl} 02 / 3$ & $\begin{array}{l}\text { Lake Clifton, E-Ufer (ca. 1km N of } \\
\text { walkway Mt. John Road), pond }\end{array}$ & $+/-0 \mathrm{~cm}$ & $\begin{array}{l}\text { Dunkler Biofilm auf weichem Sediment und } \\
\text { Thrombolithen }\end{array}$ & $9 / 04 / 02$ \\
\hline $\mathrm{LCl02/4}$ & $\begin{array}{l}\text { Lake Clifton, E-Ufer (ca. 1km N of } \\
\text { walkway Mt. John Road), pond }\end{array}$ & - & Weicher Biofilm, violett & $9 / 04 / 02$ \\
\hline $\mathrm{LCl02/5}$ & Lake Clifton, E-Ufer, boardwalk & $30 \mathrm{~cm}$ & $\begin{array}{l}\text { Basis konzentrischer Thrombolith, gelber Biofilm, } \\
\text { darunter grün auf hochporösem trombolithischem } \\
\text { Karbonat }\end{array}$ & $9 / 04 / 02$ \\
\hline $\mathrm{LCl02/6}$ & Lake Clifton, E-Ufer, boardwalk & $0-5 \mathrm{~cm}$ & $\begin{array}{l}\text { Top Bioherm, gelber Biofilm mit grüner Unterlage, } \\
\text { poröses Karbonat }\end{array}$ & $9 / 04 / 02$ \\
\hline $\mathrm{LCl02/7}$ & Lake Clifton, E-Ufer, boardwalk & $10-20 \mathrm{~cm}$ & Crossover, zwischen den Proben 02/6 und 02/7 & $9 / 04 / 02$ \\
\hline $\mathrm{LCl02/8}$ & Lake Clifton, E-Ufer, boardwalk & $5-30 \mathrm{~cm}$ & Rest der Proben LCl02/5-02/7 & $9 / 04 / 02$ \\
\hline $\mathrm{LCl02/9}$ & Lake Clifton, E-Ufer, boardwalk & $30-35 \mathrm{~cm}$ & $\begin{array}{l}\text { Basis kegelformiger Bioherm ("vent-like } \\
\text { opening"); orange gelber Biofilm }\end{array}$ & $9 / 04 / 02$ \\
\hline $\mathrm{LCl02/10}$ & $\begin{array}{l}\text { Lake Clifton, E-Ufer, boardwalk, } \\
\text { picket }\end{array}$ & $-1-5 \mathrm{~cm}$ & Biofilmüberzug am Holzsteg, gelb-orange & $9 / 04 / 02$ \\
\hline $\mathrm{LCl02/11}$ & $\begin{array}{l}\text { Lake Clifton, E-Ufer, boardwalk, } \\
\text { picket }\end{array}$ & $+/-0$ & Biofilmüberzug am Holzsteg, grünlich & $9 / 04 / 02$ \\
\hline $\mathrm{LCl} 02 / 12$ & $\begin{array}{l}\text { Lake Clifton, E-Ufer, boardwalk, } \\
\text { picket }\end{array}$ & - & Biofilmüberzug am Holzsteg, hellgrau, weiß & $9 / 04 / 02$ \\
\hline $\mathrm{LCl} 2 / \mathrm{A}$ & Lake Clifton, E-Ufer, boardwalk & $0-5 \mathrm{~cm}$ & $\begin{array}{l}\text { Lila-pinkfarbene Matte (schwimmt auf dem } \\
\text { Wasser als dm-große Blöcke), enthält Schalen } \\
\text { und Sediment }\end{array}$ & $26 / 04 / 02$ \\
\hline LCl02/B & Lake Clifton, E-Ufer, boardwalk & $+/-0 \mathrm{~cm}$ & Entspricht 02/A, trocken & $6 / 05 / 02$ \\
\hline LTh02/0 & Lake Thetis, S-Ufer & $0-5 \mathrm{~cm}$ & $\begin{array}{l}\text { Grüner Biofilm, nodulare Oberfläche vom } \\
\text { lateralen Saum um Bioherm }\end{array}$ & $12 / 04 / 02$ \\
\hline LTh02/1 & Lake Thetis, S-Ufer & $+-0 \mathrm{~cm}$ & Weißer Biofilm auf Bioerherm & $12 / 04 / 02$ \\
\hline LTh02/2 & Lake Thetis, S-Ufer & $0-5 \mathrm{~cm}$ & $\begin{array}{l}\text { Grüner Biofilm auf knotiger Stromatolith- } \\
\text { Oberfläche }\end{array}$ & $12 / 04 / 02$ \\
\hline LTh02/3 & Lake Thetis, S-Ufer & $5-10 \mathrm{~cm}$ & Gelb-orangener Biofilm Stromatolith-Oberfläche & $12 / 04 / 02$ \\
\hline LTh02/4 & Lake Thetis, S-Ufer & $0-10 \mathrm{~cm}$ & Gelb-orangener Biofilm Stromatolith-Oberfläche & $12 / 04 / 02$ \\
\hline LTh02/5 & Lake Thetis, S-Ufer & $1,8 \mathrm{~m}$ & $\begin{array}{l}\text { Bakterien-Suspension aus dem Seebecken, } \\
\text { violette Lage auf weichem Sediment }\end{array}$ & $12 / 04 / 02$ \\
\hline LTh02/6 & Lake Thetis, S-Ufer & $10 \mathrm{~cm}$ & $\begin{array}{l}\text { Stromatolith, mit orangefarbenem Biofilm, } \\
\text { zwischen den Biohermen }\end{array}$ & $12 / 04 / 02$ \\
\hline LTh02/7 & Lake Thetis, S-Ufer & $5 \mathrm{~cm}$ & $\begin{array}{l}\text { Kleiner Stromatolith, grüne Oberfläche, nahe der } \\
\text { Wasserlinie }\end{array}$ & $12 / 04 / 02$ \\
\hline LTh02/8 & Lake Thetis, S-Ufer & $10 \mathrm{~cm}$ & Äußere weiße knotige Kruste & $12 / 04 / 02$ \\
\hline LTh02/9 & Lake Thetis, S-Ufer & $2 \mathrm{~m}$ & flocculent mat und Sediment vom Seeboden & $13 / 04 / 02$ \\
\hline LTh02/10 & Lake Thetis, S-Ufer & $1,7 \mathrm{~m}$ & flocculent mat und Sediment vom Seeboden & $13 / 04 / 02$ \\
\hline LTh02/11 & Lake Thetis, S-Ufer & $2 \mathrm{~m}$ & flocculent mat und Sediment vom Seeboden & $13 / 04 / 02$ \\
\hline LTh02/12 & Lake Thetis, S-Ufer & $2 \mathrm{~m}$ & flocculent mat und Sediment vom Seeboden & $13 / 04 / 02$ \\
\hline LTh02/13 & Lake Thetis, S-Ufer & - & $\begin{array}{l}\text { Handstück, alte Stromatolith-Generation, } \\
\text { Erosionsfläche }\end{array}$ & $13 / 04 / 02$ \\
\hline LTh02/14 & Lake Thetis, S-Ufer & - & $\begin{array}{l}\text { Handstück, alte Stromatolith-Generation, } \\
\text { Erosionsfläche }\end{array}$ & $13 / 04 / 02$ \\
\hline LTh02/15 & Lake Thetis, S-Ufer & - & Zwischenschicht, Karbonatsand & $13 / 04 / 02$ \\
\hline LTh02/16 & Lake Thetis, S-Ufer & - & $\begin{array}{l}\text { Zwischenschicht, Karbonatsande, alte Stroma.- } \\
\text { Generation, zwischen Top und Basis }\end{array}$ & $13 / 04 / 02$ \\
\hline LTh02/17 & Lake Thetis, S-Ufer & - & Handstück, Stromatolith, oberer Teil & $13 / 04 / 02$ \\
\hline
\end{tabular}




\begin{tabular}{|c|c|c|c|c|}
\hline LTh02/18 & Lake Thetis, N-Ufer & & Handstück, Stromatolith-Top, Uferlinie HW & $14 / 04 / 02$ \\
\hline LTh02/19 & Lake Thetis, N-Ufer & & Kleines Handstück, z.T. lagig (like "Sichelzellen") & $14 / 04 / 02$ \\
\hline LTh02/19a & Lake Thetis, NE-Ufer & & Kleines Handstück, z.T. lagig (like "Sichelzellen") & $14 / 04 / 02$ \\
\hline LTh02/20 & Lake Thetis, S-Ufer & $2 \mathrm{~m}$ & $\begin{array}{l}\text { Flocculent mat, gefroren und gefriergetrocknet für } \\
\text { Biomarker-Analysen }\end{array}$ & $13 / 04 / 02$ \\
\hline LTh02/A & Lake Thetis, S-Ufer & $+/-0 \mathrm{~cm}$ & Weißer Biofilm, Stromatolith-Top & $14 / 04 / 02$ \\
\hline LTh02/B & Lake Thetis, S-Ufer & $0-5 \mathrm{~cm}$ & Grüner Biofilm, knotige Oberfläche, Wasselinie & $14 / 04 / 02$ \\
\hline LTh02/C & Lake Thetis, S-Ufer & $5-10 \mathrm{~cm}$ & Orangener Biofilm, lateraler Saum & $14 / 04 / 02$ \\
\hline SB02/1 & $\begin{array}{l}\text { Shark Bay, Telegraph Station, } \\
\text { Hamelin Pool }\end{array}$ & $\begin{array}{l}5 \mathrm{~cm} \\
(\mathrm{NW})\end{array}$ & $\begin{array}{l}\text { Grüne knubbelige Karbonat-Kruste an } \\
\text { supratidalem Stromatolith, } 20 \text { m von der Küste } \\
\text { entfernt }\end{array}$ & $18 / 04 / 02$ \\
\hline
\end{tabular}




\section{Anhang 3}

Probenpunkte (LTh 98/13-51 bis 63) der Kohlenstoff- und Sauerstoff-Isotope der Wachstumsphasen der äußeren Stromatolith-Kruste vom Lake Thetis.

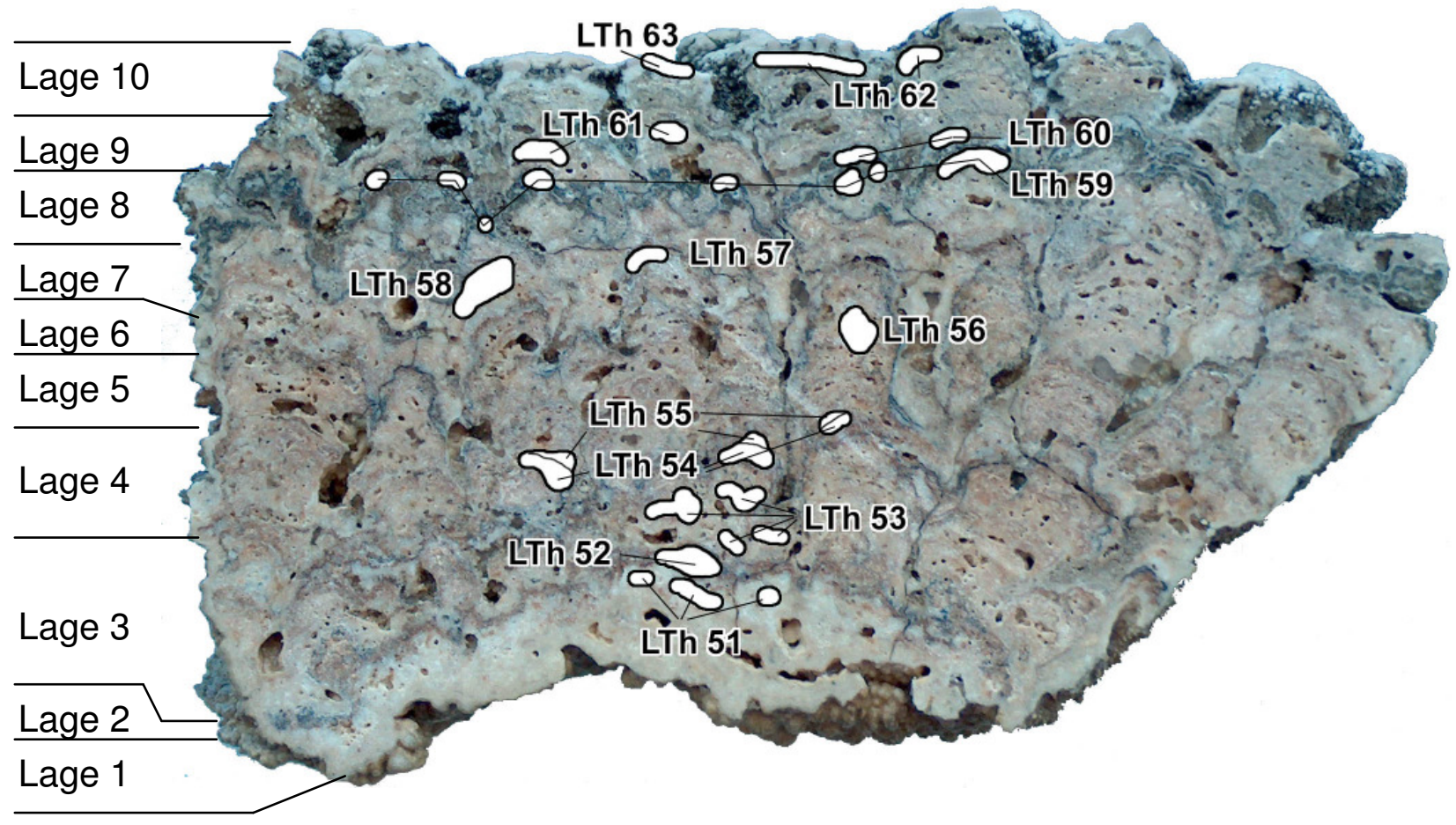

\section{Anhang 4}

Wachstumsphasen der äußeren Stromatolith-Kruste vom Lake Thetis. Die Nummern geben die Probenpunkte der Isotopen-Messungen $\left(\delta^{13} \mathrm{C}, \delta^{18} \mathrm{O}\right.$; LTh $98 / 13-2$ bis 12) an.

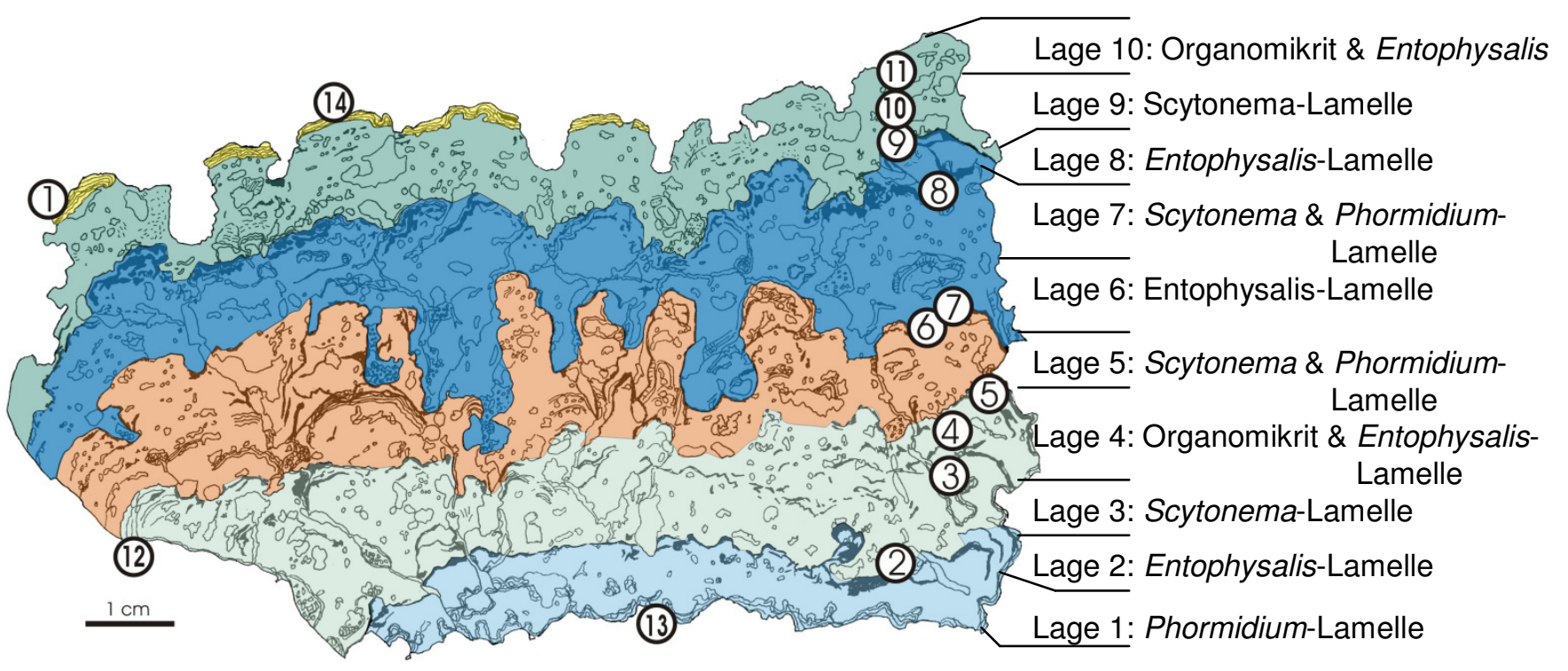


Anhang 5: Ergebnisse der Isotopen-Analysen.

\begin{tabular}{|c|c|c|c|c|c|c|c|}
\hline \multicolumn{2}{|c|}{$\begin{array}{l}\text { Isotopen-Proben-Gelände-Proben- } \\
\text { nummer }\end{array}$} & Material & & $\begin{array}{c}\delta 13 C \\
\text { Mean } \\
\text { permil V-PD }\end{array}$ & $\begin{array}{l}\text { S13C } \\
\text { Std.Abw. } \\
\text { B }\end{array}$ & $\begin{array}{c}\delta 180 \\
\text { Mean } \\
\text { permil V-PD }\end{array}$ & $\begin{array}{l}8180 \\
\text { Std.Abw. } \\
\text { DB }\end{array}$ \\
\hline \multicolumn{8}{|c|}{ Scytonema-Lagen } \\
\hline LTh 3 & LTh $98 / 13$ & $\begin{array}{l}\text { subfossiler Stromatolith, } \\
\text { äußere Kruste }\end{array}$ & Scytonema -Lamelle + faserige Aragonitzemente & 2,17 & 0,01 & 2,23 & 0,01 \\
\hline LTh 4 & LTh 98/13 & $\begin{array}{l}\text { subfossiler Stromatolith, } \\
\text { äußere Kruste }\end{array}$ & Scytonema -Lamelle + faserige Aragonitzemente & 1,89 & 0,01 & 2,69 & 0,01 \\
\hline LTh 6 & LTh $98 / 13$ & $\begin{array}{l}\text { subfossiler Stromatolith, } \\
\text { äußere Kruste }\end{array}$ & Scytonema -Lamelle + faserige Aragonitzemente & 2,04 & 0,01 & 2,23 & 0,01 \\
\hline LTh 8 & LTh $98 / 13$ & $\begin{array}{l}\text { subfossiler Stromatolith, } \\
\text { äußere Kruste }\end{array}$ & Scytonema -Lamelle + faserige Aragonitzemente & 2,18 & 0,01 & 2,12 & 0,01 \\
\hline LTh 10 & LTh $98 / 13$ & $\begin{array}{l}\text { subfossiler Stromatolith, } \\
\text { äußere Kruste }\end{array}$ & Scytonema -Lamelle + faserige Aragonitzemente & 1,50 & 0,01 & 1,84 & 0,02 \\
\hline LTh 11 & LTh 98/13 & $\begin{array}{l}\text { subfossiler Stromatolith, } \\
\text { äußere Kruste }\end{array}$ & Scytonema -Lamelle + allochthoner Detritus und Mikrite von Taschen & 1,78 & 0,01 & 1,66 & 0,01 \\
\hline LTh 5 & LTh $98 / 13$ & $\begin{array}{l}\text { subfossiler Stromatolith, } \\
\text { äußere Kruste }\end{array}$ & $\begin{array}{l}\text { Top einer Scytonema -Phormidium -Lamelle + darauffolgende Organomikrit- } \\
\text { Lage }\end{array}$ & 2,29 & 0,01 & 2,58 & 0,01 \\
\hline LTh 53 & LTh $98-13$ & $\begin{array}{l}\text { subfossiler Stromatolith, } \\
\text { äußere Kruste }\end{array}$ & $\begin{array}{l}\text { Stromatolith (äußere Kruste), dritte Lage: weißgraue, feinfaserige } \\
\text { Aragonitlamelle mit erekten, dicken Filamenten (Scytonema -Lage) }\end{array}$ & 3,46 & 0,01 & 2,54 & 0,03 \\
\hline LTh 56 & LTh 98-13 & $\begin{array}{l}\text { subfossiler Stromatolith, } \\
\text { äußere Kruste }\end{array}$ & $\begin{array}{l}\text { Stromatolith (äußere Kruste), fünfte Lage: weißgraue, feinfaserige } \\
\text { Aragonitlamelle mit erekten, dicken Filamenten (Scytonema -Lage) }\end{array}$ & 2,60 & 0,02 & 3,04 & 0,02 \\
\hline LTh 58 & LTh $98-13$ & $\begin{array}{l}\text { subfossiler Stromatolith, } \\
\text { äußere Kruste }\end{array}$ & $\begin{array}{l}\text { Stromatolith (äußere Kruste), siebte Lage: weißgraue, feinfaserige } \\
\text { Aragonitlamelle mit erekten, dicken Filamenten (Scytonema-Lage) }\end{array}$ & 2,93 & 0,02 & 2,80 & 0,03 \\
\hline LTh 61 & LTh $98-13$ & $\begin{array}{l}\text { subfossiler Stromatolith, } \\
\text { äußere Kruste }\end{array}$ & $\begin{array}{l}\text { Stromatolith (äußere Kruste), neunte Lage: weißgraue, feinfaserige } \\
\text { Aragonitlamelle mit erekten, dicken Filamenten (Scytonema -Lage) }\end{array}$ & 1,89 & 0,02 & 2,15 & 0,01 \\
\hline LTh 27 & LTh $02 / 19$ & $\begin{array}{l}\text { subfossiler Stromatolith, } \\
\text { äußere Kruste (stark } \\
\text { angewittert) }\end{array}$ & abschließender Scytonema -Stromatolith + faserige Aragonitzemente & 0,73 & 0,01 & 2,26 & 0,01 \\
\hline \multicolumn{8}{|c|}{ Entophysalis-Lagen } \\
\hline LTh 2 & LTh 98/13 & $\begin{array}{l}\text { subfossiler Stromatolith, } \\
\text { äußere Kruste }\end{array}$ & Entophysalis -Lamelle (abschließendes dunkles Band von Teilphase A) & 1,80 & 0,01 & 2,27 & 0,02 \\
\hline LTh 7 & LTh $98 / 13$ & $\begin{array}{l}\text { subfossiler Stromatolith, } \\
\text { äußere Kruste }\end{array}$ & Entophysalis -Lamelle + angrenzende Phormidium-Scytonema-Partien & 2,53 & 0,01 & 2,51 & 0,02 \\
\hline LTh 9 & LTh $98 / 13$ & $\begin{array}{l}\text { subfossiler Stromatolith, } \\
\text { äußere Kruste }\end{array}$ & Entophysalis -Lamelle + punktuell Organomikritkrusten & 2,31 & 0,02 & 2,04 & 0,02 \\
\hline LTh 52 & LTh $98-13$ & $\begin{array}{l}\text { subfossiler Stromatolith, } \\
\text { äußere Kruste }\end{array}$ & $\begin{array}{l}\text { Stromatolith (äußere Kruste), zweite Lage: braune bis grünlich-schwarze, } \\
\text { feinkristalline Aragonitlamelle mit Relikten kokkaler Mikroorganismen } \\
\text { (Entophysalis -Lage) }\end{array}$ & 2,31 & 0,03 & 2,37 & 0,02 \\
\hline LTh 54 & LTh $98-13$ & $\begin{array}{l}\text { subfossiler Stromatolith, } \\
\text { äußere Kruste }\end{array}$ & $\begin{array}{l}\text { Stromatolith (äußere Kruste), vierte Lage: braune bis grünlich-schwarze } \\
\text { feinkristalline Aragonitlamelle mit Relikten kokkaler Mikroorganismen } \\
\text { (Entophysalis -Lage) }\end{array}$ & 2,55 & 0,02 & 2,85 & 0,02 \\
\hline LTh 57 & LTh $98-13$ & $\begin{array}{l}\text { subfossiler Stromatolith, } \\
\text { äußere Kruste }\end{array}$ & $\begin{array}{l}\text { Stromatolith (äußere Kruste), sechste Lage: dünne, braune bis grünlich- } \\
\text { schwarze, feinkristalline Aragonitlamelle mit Relikten kokkaler } \\
\text { Mikroorganismen (Entophysalis -Lage+abschließende Faseraragonitlage) }\end{array}$ & 2,63 & 0,02 & 3,02 & 0,03 \\
\hline LTh 59 & LTh $98-13$ & $\begin{array}{l}\text { subfossiler Stromatolith, } \\
\text { äußere Kruste }\end{array}$ & $\begin{array}{l}\text { Stromatolith (äußere Kruste), achte Lage: braune bis grünlich-schwarze } \\
\text { feinkristalline Aragonitlamelle mit Relikten kokkaler Mikroorganismen } \\
\text { (Entophysalis -Lage) }\end{array}$ & 2,47 & 0,01 & 2,32 & 0,02 \\
\hline LTh 62 & LTh 98-13 & $\begin{array}{l}\text { subfossiler Stromatolith, } \\
\text { äußere Kruste }\end{array}$ & $\begin{array}{l}\text { Stromatolith (äußere Kruste), zehnte Lage: dünne braune bis grünlich- } \\
\text { schwarze feinkristalline Aragonitlamelle mit Relikten kokkaler } \\
\text { Mikroorganismen (Entophysalis -Lage) }\end{array}$ & 2,99 & 0,02 & 2,11 & 0,03 \\
\hline \multicolumn{8}{|c|}{ Phormidium -Lagen } \\
\hline LTh 51 & LTh $98-13$ & $\begin{array}{l}\text { subfossiler Stromatolith, } \\
\text { äußere Kruste }\end{array}$ & $\begin{array}{l}\text { Stromatolith (äußere Kruste), erste Lage: weißgraue, einschlußreiche } \\
\text { feinfaserige Aragonitlamelle mit wenigen dünnen erekten Filamentspuren } \\
\text { (Phormidium -Lage) }\end{array}$ & 1,71 & 0,02 & 2,29 & 0,03 \\
\hline LTh 21 & LTh $98 / 12$ & $\begin{array}{l}\text { subfossiler Stromatolith vom } \\
\text { Uferbereich, äußere Kruste }\end{array}$ & Phormidium -Stromatolith (faseriger einschlußreicher Aragonit) & 2,76 & 0,01 & 2,39 & 0,02 \\
\hline LTh 17 & LTh $98 / 14$ & $\begin{array}{l}\text { subfossiler Stromatolith, } \\
\text { mittlere Kruste }\end{array}$ & $\begin{array}{l}\text { tiefer Teil Gesamtgestein (Phormidium -Aragonit, allochthone Bioklasten und } \\
\text { Pellets, Organomikritkrusten) }\end{array}$ & 1,88 & 0,01 & 2,39 & 0,01 \\
\hline LTh 18 & LTh $98 / 14$ & $\begin{array}{l}\text { subfossiler Stromatolith, } \\
\text { mittlere Kruste }\end{array}$ & $\begin{array}{l}\text { mittlerer Teil Gesamtgestein (Phormidium -Aragonit, allochthone Bioklasten } \\
\text { und Pellets, Organomikritkrusten) }\end{array}$ & 2,41 & 0,01 & 2,08 & 0,01 \\
\hline \multicolumn{8}{|c|}{ Phormidium -Scytonema } \\
\hline LTh 23 & LTh 98/15 & $\begin{array}{l}\text { subfossiler Stromatolith, } \\
\text { subaquatische Ebene } \\
\text { zwischen Stromatolithstotzen, } \\
\text { äußere Kruste }\end{array}$ & unterer Teil aus Phormidium -Scytonema-Kruste & 2,44 & 0,01 & 1,96 & 0,01 \\
\hline LTh 19 & LTh $98 / 14$ & $\begin{array}{l}\text { subfossiler Stromatolith, } \\
\text { mittlere Kruste }\end{array}$ & $\begin{array}{l}\text { oberer Teil Gesamtgestein (Phormidium -Scytonema -Aragonit, allochthone } \\
\text { Bioklasten und Pellets, Organomikritkrusten) }\end{array}$ & 2,49 & 0,01 & 2,21 & 0,01 \\
\hline LTh 20 & LTh $98 / 12$ & $\begin{array}{l}\text { subfossiler Stromatolith vom } \\
\text { Uferbereich, äußere Kruste }\end{array}$ & $\begin{array}{l}\text { mikritische Pellets in mikritischer bis mikropeloidaler Matrix aus } \\
\text { Taschenfüllung; makroskopisch grau und bröselig }\end{array}$ & 0,79 & 0,01 & 2,55 & 0,01 \\
\hline \multicolumn{8}{|c|}{ Phormidium-Entophysalis } \\
\hline LTh 24 & LTh 98/15 & $\begin{array}{l}\text { subfossiler Stromatolith, } \\
\text { subaquatische Ebene } \\
\text { zwischen Stromatolithstotzen, } \\
\text { äußere Kruste }\end{array}$ & $\begin{array}{l}\text { oberer Teil aus Phormidium-Entophysalis-Kruste, allochthonen Ooiden und } \\
\text { Pellets (Taschen) und abschließender Organomikritkruste (von Endolithen } \\
\text { zerbohrt) }\end{array}$ & 2,34 & 0,01 & 2,01 & 0,02 \\
\hline \multicolumn{8}{|c|}{ Organomikritkruste } \\
\hline LTh 1 & LTh $98 / 13$ & $\begin{array}{l}\text { subfossiler Stromatolith, } \\
\text { äußere Kruste }\end{array}$ & $\begin{array}{l}\text { abschließende Organomikritkruste (faseriger und mikrokristalliner Aragonit } \\
\text { im Wechsel) }\end{array}$ & 1,37 & 0,01 & 2,26 & 0,01 \\
\hline LTh 25 & LTh $02 / 14$ & $\begin{array}{l}\text { subfossiler Stromatolith, } \\
\text { äußere Kruste }\end{array}$ & $\begin{array}{l}\text { abschließende Organomikritkruste (faseriger und mikrokristalliner Aragonit } \\
\text { im Wechsel) + angrenzende unterlagernde Stromatolith-Lamelle } \\
\text { (?Phormidium -Scytonema mit allochthonen Detritus) }\end{array}$ & 2,54 & 0,01 & 1,85 & 0,01 \\
\hline LTh 16 & LTh $98 / 14$ & $\begin{array}{l}\text { subfossiler Stromatolith, } \\
\text { mittlere Kruste }\end{array}$ & $\begin{array}{l}\text { äußere Organomikritkruste (faseriger und mikrokristalliner Aragonit im } \\
\text { Wechsel) }\end{array}$ & 2,14 & 0,01 & 2,41 & 0,02 \\
\hline LTh 12 & LTh $98 / 13$ & $\begin{array}{l}\text { subfossiler Stromatolith, } \\
\text { äußere Kruste }\end{array}$ & $\begin{array}{l}\text { abschließende Organomikritkruste (faseriger und mikrokristalliner Aragonit } \\
\text { im Wechsel)) }\end{array}$ & 2,28 & 0,01 & 1,22 & 0,02 \\
\hline LTh 13 & LTh $98 / 13$ & $\begin{array}{l}\text { subfossiler Stromatolith, } \\
\text { äußere Kruste }\end{array}$ & $\begin{array}{l}\text { abschließende Organomikritkruste (faseriger und mikrokristalliner Aragonit } \\
\text { im Wechsel)) }\end{array}$ & 1,81 & 0,01 & 1,99 & 0,01 \\
\hline LTh 14 & LTh $98 / 13$ & $\begin{array}{l}\text { subfossiler Stromatolith, } \\
\text { äußere Kruste }\end{array}$ & $\begin{array}{l}\text { abschließende Organomikritkruste (faseriger und mikrokristalliner Aragonit } \\
\text { im Wechsel)) }\end{array}$ & 2,46 & 0,01 & 1,93 & 0,02 \\
\hline LTh 55 & LTh $98-13$ & $\begin{array}{l}\text { subfossiler Stromatolith, } \\
\text { äußere Kruste }\end{array}$ & $\begin{array}{l}\text { Stromatolith (äußere Kruste), vierte Lage: abschließende weiße, faserige } \\
\text { Aragonitlamelle ("Organomikrit") }\end{array}$ & 1,98 & 0,03 & 2,01 & 0,02 \\
\hline LTh 60 & LTh $98-13$ & $\begin{array}{l}\text { subfossiler Stromatolith, } \\
\text { äußere Kruste }\end{array}$ & $\begin{array}{l}\text { Stromatolith (äußere Kruste), achte Lage: abschließende weiße, faserige } \\
\text { Aragonitlamelle ("Organomikrit") }\end{array}$ & 2,80 & 0,02 & 2,25 & 0,02 \\
\hline LTh 63 & LTh $98-13$ & $\begin{array}{l}\text { subfossiler Stromatolith, } \\
\text { äußere Kruste }\end{array}$ & $\begin{array}{l}\text { Stromatolith (äußere Kruste), zehnte Lage: abschließende weiße, faserig- } \\
\text { mikrokristalline Aragonitlamelle ("Organomikrit") }\end{array}$ & 1,72 & 0,04 & 1,56 & 0,04 \\
\hline
\end{tabular}


Fortsetzung Anhang 5

\begin{tabular}{|c|c|c|c|c|c|c|c|}
\hline $\begin{array}{c}\text { Isotopen } \\
\text { Probennummer }\end{array}$ & $\begin{array}{c}\text { Geländeproben- } \\
\text { Nummer }\end{array}$ & Material & & $\begin{array}{c}\delta 13 \mathrm{C} \\
\text { Mean } \$ \\
\text { permil V-PDB }\end{array}$ & 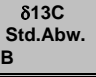 & $\begin{array}{c}\delta 180 \\
\text { Mean } S \\
\text { permil V-PDB }\end{array}$ & $\begin{array}{l}\text { S180 } \\
\text { Std.Abw. } \\
\text { B }\end{array}$ \\
\hline \multicolumn{8}{|c|}{ Ostrakoden Kern JR } \\
\hline LTh 33 & LT 1-3 & $\begin{array}{l}\text { Ostrakoden (9 Klappen einer } \\
\text { Art) }\end{array}$ & & $-6,95$ & 0,01 & 1,36 & 0,03 \\
\hline LTh 34 & LT 1-4 & $\begin{array}{l}\text { Ostrakoden (10 Klappen einer } \\
\text { Art) }\end{array}$ & & $-4,60$ & 0,01 & 1,49 & 0,01 \\
\hline LTh 36 & LT 1-5 & $\begin{array}{l}\text { Ostrakoden (8 Klappen einer } \\
\text { Art) }\end{array}$ & & $-5,45$ & 0,03 & 1,55 & 0,03 \\
\hline LTh 38 & LT 1-6 & $\begin{array}{l}\text { Ostrakoden (8 Klappen einer } \\
\text { Art) }\end{array}$ & & $-4,75$ & 0,03 & 1,34 & 0,01 \\
\hline LTh 43 & LT 2-3 & $\begin{array}{l}\text { Ostrakoden (7 Klappen einer } \\
\text { Art) }\end{array}$ & & $-4,88$ & 0,03 & 1,80 & 0,04 \\
\hline LTh 45 & LT 2-4 & $\begin{array}{l}\text { Ostrakoden (8 Klappen einer } \\
\text { Art) }\end{array}$ & & $-3,60$ & 0,01 & 2,58 & 0,02 \\
\hline LTh 47 & LT 3-1 & $\begin{array}{l}\text { Ostrakoden (6 Klappen einer } \\
\text { Art) }\end{array}$ & & $-4,92$ & 0,02 & 0,55 & 0,01 \\
\hline LTh 49 & LT 3-2 & $\begin{array}{l}\text { Ostrakoden (7 Klappen einer } \\
\text { Art) }\end{array}$ & & $-4,39$ & 0,02 & 1,25 & 0,02 \\
\hline \multicolumn{8}{|c|}{ Spherulite Sedimentkern JR } \\
\hline LTh 39 & LT 1-7 & $\begin{array}{l}\text { Sedimentkern Lake Thetis: } \\
\text { kleine weiße } \\
\text { Karbonataggregate }\end{array}$ & & $-2,61$ & 0,02 & 2,25 & 0,03 \\
\hline LTh 40 & LT 2-1 & $\begin{array}{l}\text { Sedimentkern Lake Thetis: } \\
\text { weiße Karbonataggregate }\end{array}$ & & 1,56 & 0,02 & 2,22 & 0,03 \\
\hline LTh 41 & LT 2-2 & $\begin{array}{l}\text { Sedimentkern Lake Thetis: } \\
\text { weiße Karbonataggregate }\end{array}$ & & $-3,63$ & 0,02 & 2,98 & 0,02 \\
\hline LTh 42 & LT 2-3 & $\begin{array}{l}\text { Sedimentkern Lake Thetis: } \\
\text { weiße Sphärolithaggregate }\end{array}$ & & $-0,38$ & 0,03 & 2,16 & 0,02 \\
\hline LTh 44 & LT 2-4 & $\begin{array}{l}\text { Sedimentkern Lake Thetis: } \\
\text { orangebraune-weiße, gut } \\
\text { ausgebildete Sphärolithe }\end{array}$ & & 0,49 & 0,02 & 2,82 & 0,01 \\
\hline LTh 37 & LT 1-6 & $\begin{array}{l}\text { Sedimentkern Lake Thetis: } \\
\text { kleine weiße } \\
\text { Karbonataggregate }\end{array}$ & & 1,12 & 0,02 & 2,25 & 0,01 \\
\hline LTh 48 & LT 3-2 & $\begin{array}{l}\text { Sedimentkern Lake Thetis: } \\
\text { kleine weiße } \\
\text { Karbonataggregate }\end{array}$ & & 0,16 & 0,02 & 2,37 & 0,01 \\
\hline LTh 50 & LT 3-3 & $\begin{array}{l}\text { Sedimentkern Lake Thetis: } \\
\text { kleine weiße } \\
\text { Karbonataggregate }\end{array}$ & & $-2,39$ & 0,01 & 1,86 & 0,02 \\
\hline LTh 46 & LT 3-1 & $\begin{array}{l}\text { Sedimentkern Lake Thetis: } \\
\text { weiße Sphärolithaggregate }\end{array}$ & & 3,12 & 0,00 & 2,65 & 0,02 \\
\hline LTh 35 & LT 1-5 & $\begin{array}{l}\text { Sedimentkern Lake Thetis: } \\
\text { Sphärolithe (klein, } \\
\text { orangebraun) }\end{array}$ & & $-2,29$ & 0,01 & 3,01 & 0,02 \\
\hline LTh 31 & LT 1-1 & $\begin{array}{l}\text { Sedimentkern Lake Thetis: } \\
\text { Sphärolithe (weiß) }\end{array}$ & & $-4,84$ & 0,02 & 2,68 & 0,03 \\
\hline LTh 32 & LT 1-3 & $\begin{array}{l}\text { Sedimentkern Lake Thetis: } \\
\text { Sphärolithe (klein, } \\
\text { orangebraun) }\end{array}$ & & $-6,76$ & 0,03 & 2,58 & 0,02 \\
\hline \multicolumn{8}{|c|}{ Sedimentkern 2002} \\
\hline LTh 28 & LTh K2 1 & $\begin{array}{l}\text { Sedimentkern Seebecken, } 15 \\
\text { m vom Ufer entfernt }\end{array}$ & 0-2 cm: pinkfarbenes organisches flockiges Sediment & $-1,68$ & 0,01 & 2,19 & 0,02 \\
\hline LTh 29 & LTh K2 5 & $\begin{array}{l}\text { Sedimentkern Seebecken, } 15 \\
\text { m vom Ufer entfernt }\end{array}$ & 9-10 cm braungraues organisches flockiges Sediment & $-0,23$ & 0,01 & 2,45 & 0,01 \\
\hline LTh 30 & LTh K2 9 & $\begin{array}{l}\text { Sedimentkern Seebecken, } 15 \\
\text { m vom Ufer entfernt }\end{array}$ & $\begin{array}{l}17-18 \mathrm{~cm} \text { rotbraues organisches, gelatineartiges Sediment mit hellrotbrauner } \\
\text { Lage }\end{array}$ & $-0,95$ & 0,01 & 2,54 & 0,01 \\
\hline \multicolumn{8}{|c|}{ Gesamt ohne äußere Kruste } \\
\hline LTh 15 & LTh 98/14 & $\begin{array}{l}\text { subfossiler Stromatolith, } \\
\text { mittlere Kruste }\end{array}$ & Gesamtgestein ohne äußere Organomikritkruste & 3,19 & 0,01 & 2,53 & 0,01 \\
\hline LTh 26 & LTh $02 / 18$ & $\begin{array}{l}\text { subfossiler Stromatolith, } \\
\text { äußere Kruste }\end{array}$ & Gesamtgestein ohne äußere Organomikritkruste & 1,61 & 0,01 & 1,62 & 0,01 \\
\hline \multicolumn{8}{|c|}{ Holozäner Karbonatsand } \\
\hline LTh 22 & LTh $98 / 9$ & & $\begin{array}{l}\text { holozäner mariner Karbonatsand mit vadosen Mikritbrücken zwischen den } \\
\text { Komponenten (Rotalgen, Echinodermen, Foraminiferen, Molluskenbruch) }\end{array}$ & $-0,70$ & 0,01 & 0,59 & 0,02 \\
\hline \multicolumn{8}{|l|}{ Lake Clifton etc } \\
\hline 06/06/03 & GoePa 01-31 & $\mathrm{LCl} 02 / 2$ & fibröse Matte auf Sediment, pond & 5,00 & 0,02 & 2,25 & 0,02 \\
\hline 06/06/03 & GoePa 01-32 & $\mathrm{LCl} 02 / 3$ & schwarzer Biofilm auf thrombolith & 8,07 & 0,01 & 3,02 & 0,01 \\
\hline 06/06/03 & GoePa 01-33 & $\mathrm{LCl} 02 / 4$ & violette Biomatte & 8,00 & 0,01 & 2,86 & 0,01 \\
\hline 06/06/03 & GoePa 01-34 & $\mathrm{LCl} 02 / 5$ & Basis konzentrische Bioherme, Steg & 11,21 & 0,01 & 3,34 & 0,02 \\
\hline 06/06/03 & GoePa 01-35 & $\mathrm{LCl} 02 / 6$ & Top konzentrische Bioherme, Steg & 6,47 & 0,02 & 4,09 & 0,02 \\
\hline 06/06/03 & GoePa 01-36 & $\mathrm{LCl} 02 / 7$ & konzentrische Bioherme, Steg & 10,30 & 0,01 & 2,91 & 0,02 \\
\hline 06/06/03 & GoePa 01-37 & LCl 02/9 & Basis konzentrische Bioherme, Steg & 10,38 & 0,01 & 3,60 & 0,02 \\
\hline 06/06/03 & GoePa 01-38 & $\mathrm{LCl} 02 / \mathrm{A}$ & violette Biomatte & 6,29 & 0,03 & $-3,60$ & 0,07 \\
\hline 06/06/03 & GoePa 01-39 & LPr 98/10 & fossiler Stromatolith, unterer Teil & $-2,98$ & 0,01 & $-1,63$ & 0,01 \\
\hline 06/06/03 & GoePa 01-40 & LPr 98/10 & fossiler Stromatolith, oberer Teil & 1,93 & 0,02 & 2,43 & 0,02 \\
\hline 06/06/03 & GoePa 01-41 & LPr 98/11 & fossiler Stromatolith, mittlerer Teil & $-8,49$ & 0,01 & $-3,64$ & 0,01 \\
\hline 06/06/03 & GoePa 01-42 & LPr $98 / 12$ & fossiler Stromatolith, klastischer Teil & $-4,77$ & 0,01 & $-2,89$ & 0,02 \\
\hline 06/06/03 & GoePa 01-43 & LPr $98 / 12$ & fossiler Stromatolith, laminierter Teil & $-7,93$ & 0,01 & $-4,02$ & 0,01 \\
\hline 06/06/03 & GoePa 01-44 & LPr $02 / 03$ & Stromatolith, Bulk & 1,98 & 0,01 & 2,67 & 0,01 \\
\hline 06/06/03 & GoePa 01-45 & LPo 98/4 & Stromatolith, Bulk & 3,68 & 0,01 & 3,24 & 0,02 \\
\hline 06/06/03 & GoePa 01-46 & SB 98/18 & fossiler Stromatolith & 4,77 & 0,01 & 3,14 & 0,01 \\
\hline $06 / 06 / 03$ & GoePa 01-47 & SB 98/18 & fossiler Stromatolith & 4,62 & 0,01 & 2,61 & 0,01 \\
\hline 06/07/03 & GoePa 01-48 & SB $98 / 29$ & grüne Aufwachsungen an fossilen Stromatolith & 4,91 & 0,01 & 3,10 & 0,01 \\
\hline 06/07/03 & GoePa 01-49 & SB 98/29 & grüne Aufwachsungen (innerer Bereich)an fossilen Stromatolith & 4,87 & 0,01 & 2,99 & 0,01 \\
\hline 06/07/03 & GoePa 01-50 & Lwa 02/3 & weiße Karbonatkruste, Top, rezent & 6,66 & 0,01 & 5,65 & 0,01 \\
\hline 06/07/03 & GoePa 01-51 & Lwa 02/5 & weiße Kruste, außen, fossil & 6,70 & 0,02 & 4,29 & 0,02 \\
\hline $06 / 07 / 03$ & GoePa 01-52 & Lwa 02/6 & fosiler Stromatolith & 3,40 & 0,01 & 1,45 & 0,01 \\
\hline
\end{tabular}


Anhang 6a: Ergebnisse der RDA-Untersuchungen, Lake Thetis $3,6^{\circ} / \mathrm{min}, 0,2 \mathrm{k} \mathrm{Imp} / \mathrm{sec}, \mathrm{RTC} 2$
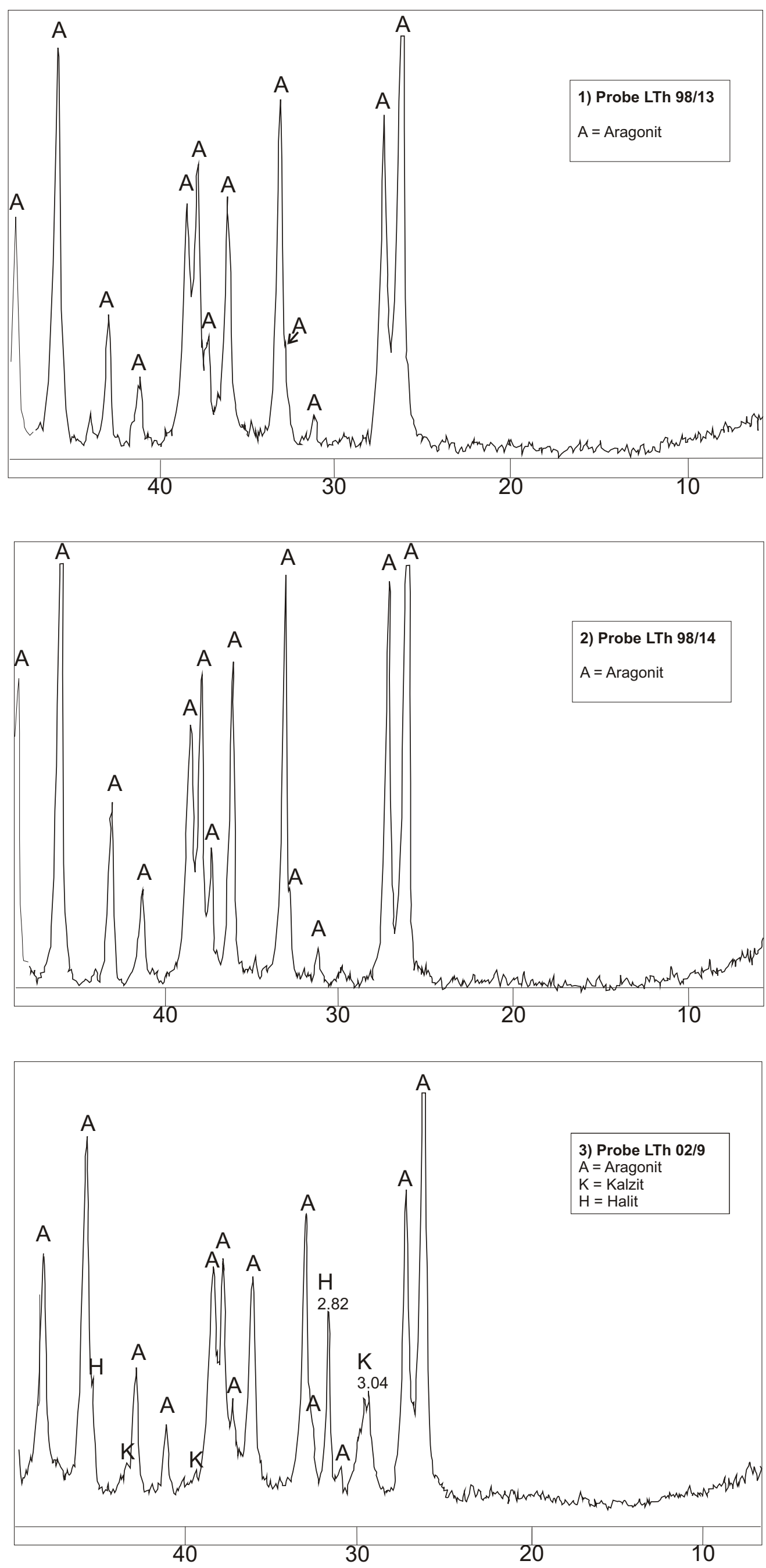
Anhang 6b: Ergebnisse der RDA-Untersuchungen, Lake Clifton und Lake Preston $3,6^{\circ} / \mathrm{min}, 0,2 \mathrm{k} \mathrm{Imp} / \mathrm{sec}$, RTC 2
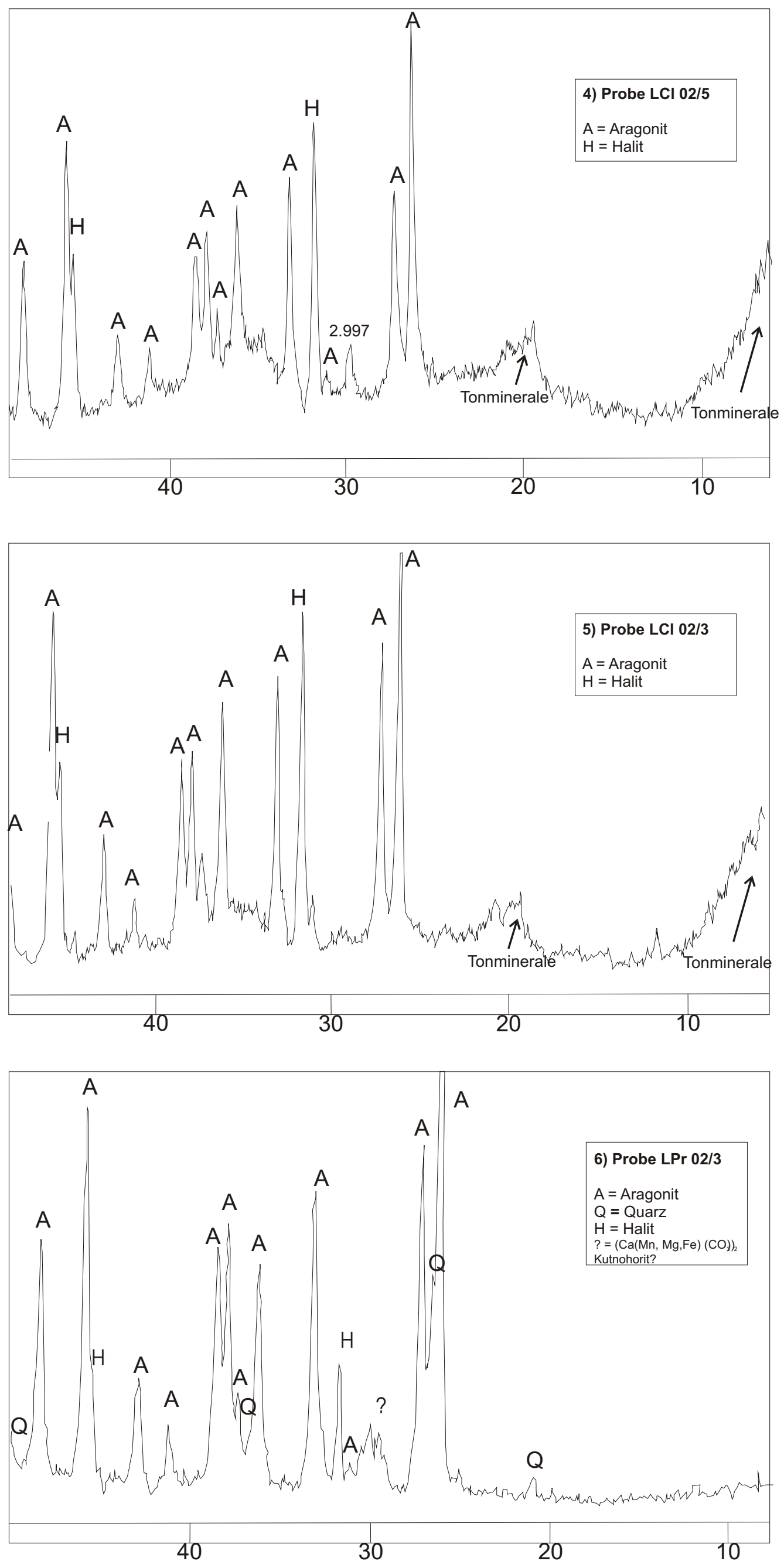
Anhang 6c: Ergebnisse der RDA-Untersuchungen, Lake Pollard und Lake Walyungup $3,6^{\circ} / \mathrm{min}, 0,2 \mathrm{k} \mathrm{Imp} / \mathrm{sec}$, RTC 2
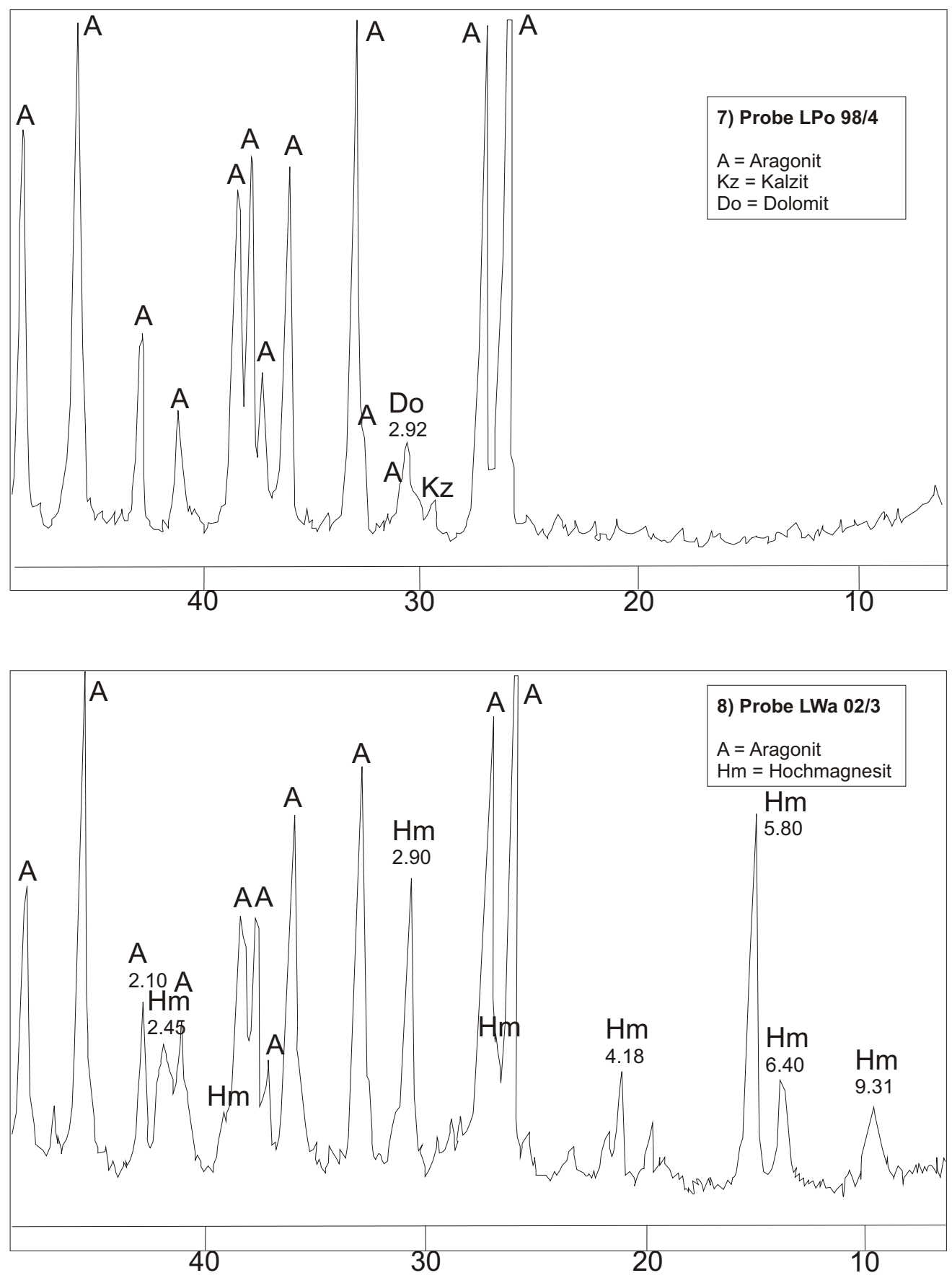
Anhang 6d: Ergebnisse der RDA-Untersuchungen, Lake Walyungup und Shark Bay $3,6^{\circ} / \mathrm{min}, 0,2 \mathrm{k} \mathrm{Imp} / \mathrm{sec}$, RTC 2
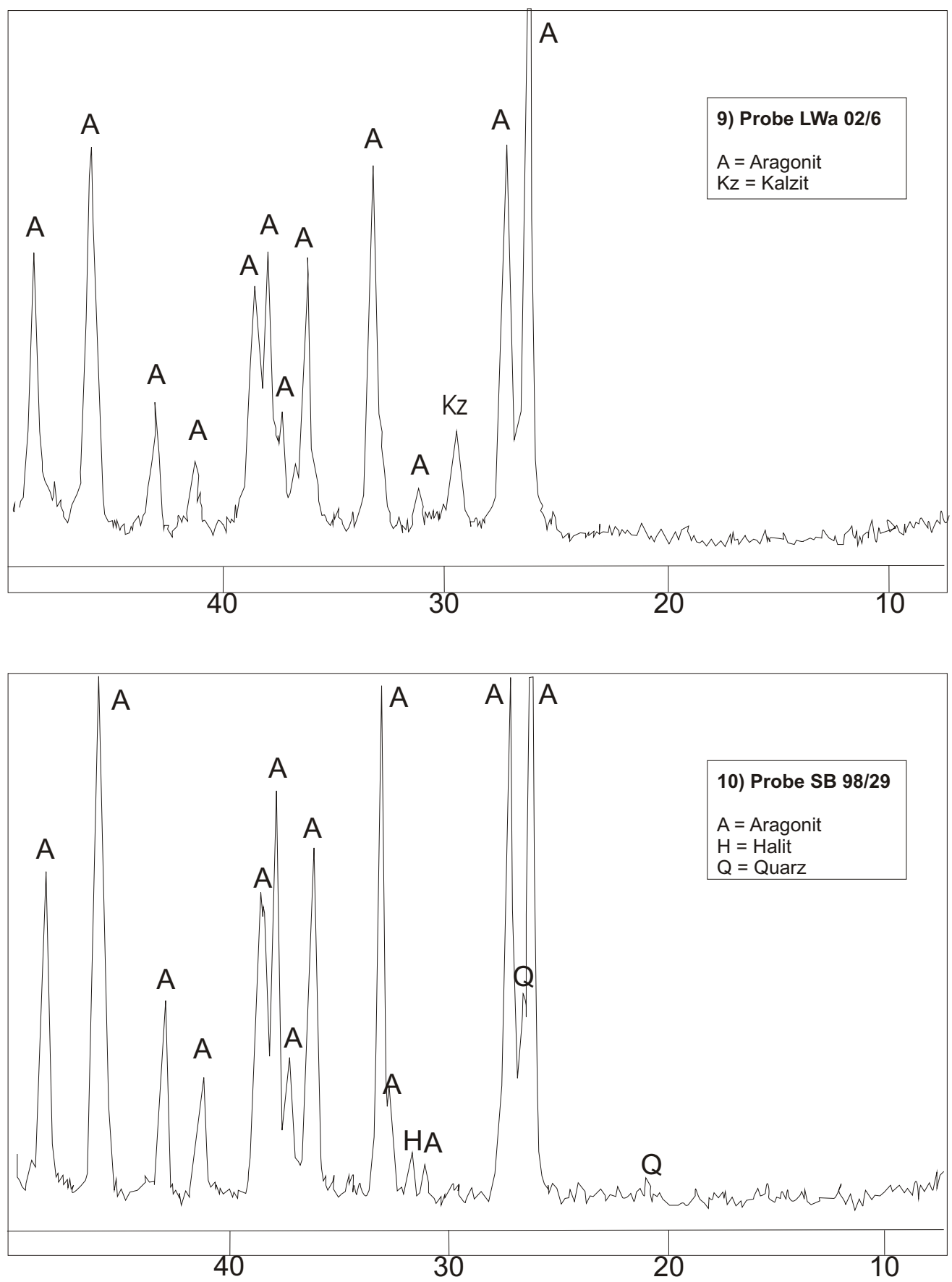


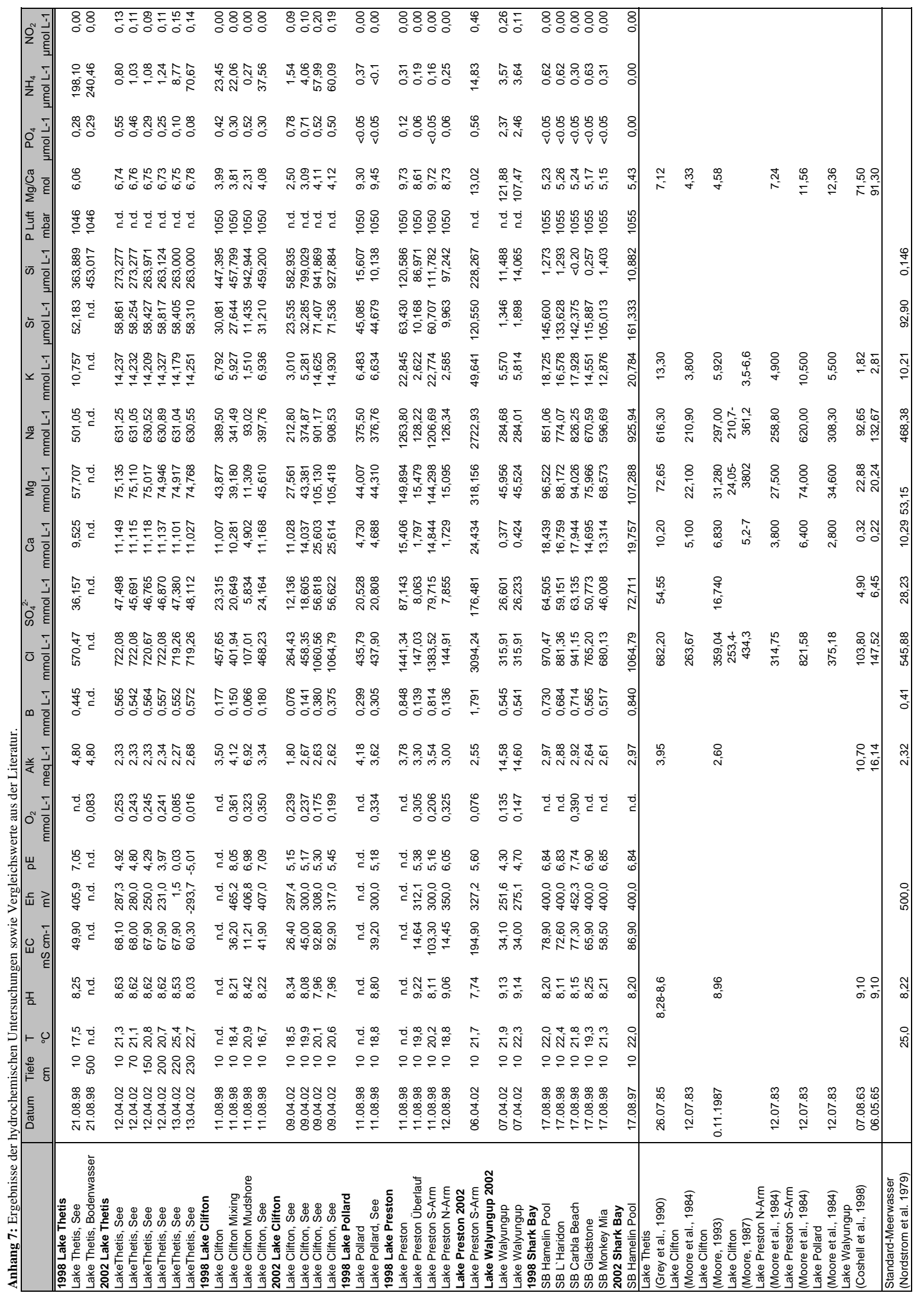


Anhang 8: Prozentualer Anteil der Haupt- und Spurenelemente in den Mikrobialithen.
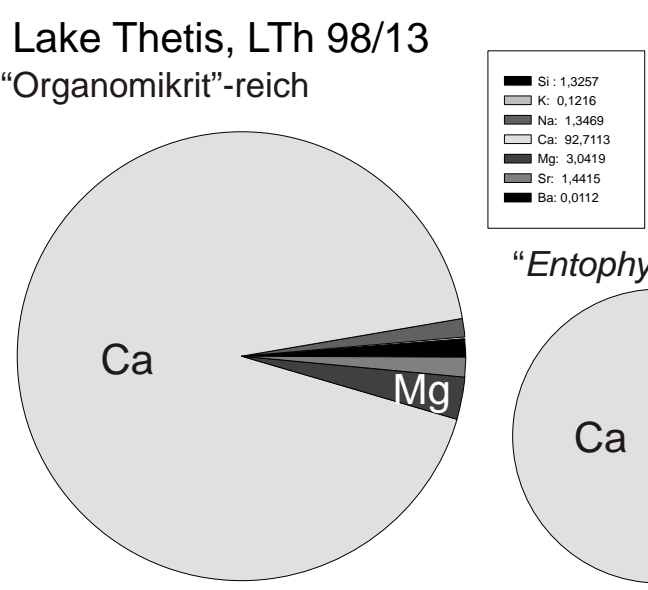

"Entophysalis-Lamelle"

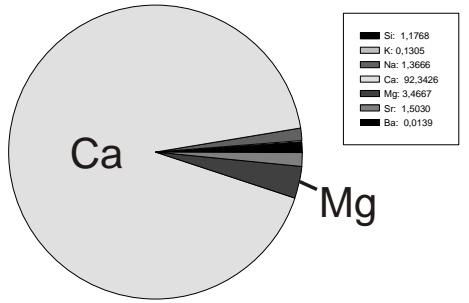

\section{LTh 98/12}

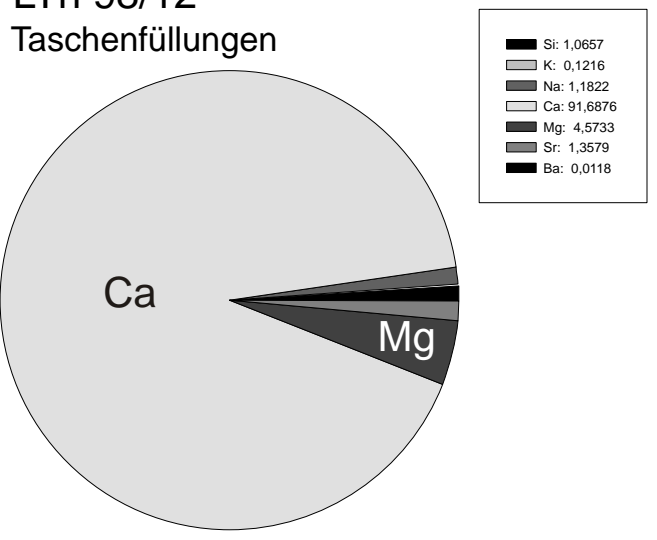

Lake Clifton, LCI 02/5

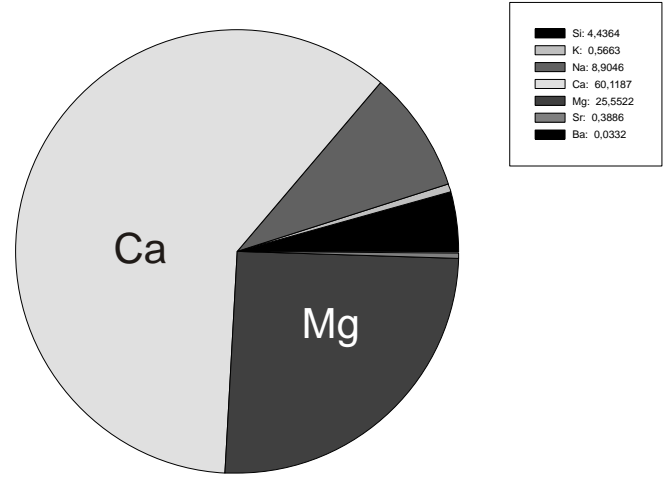

Lake Walyungup, LWa 02/5

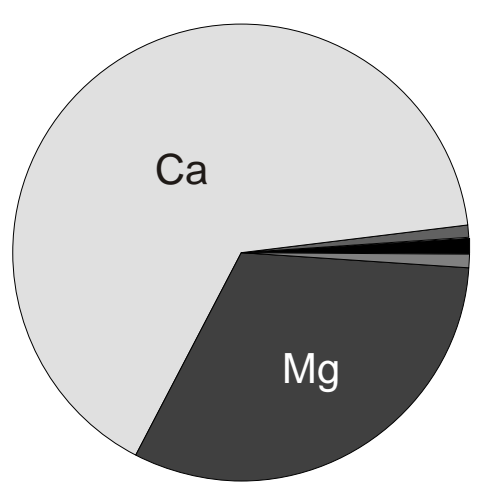

LTh 98/14

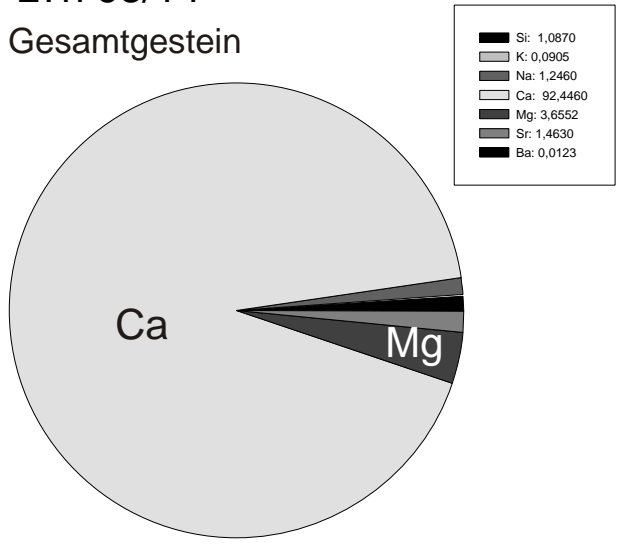

LTh $98 / 12$

"Organomikrit"-reich

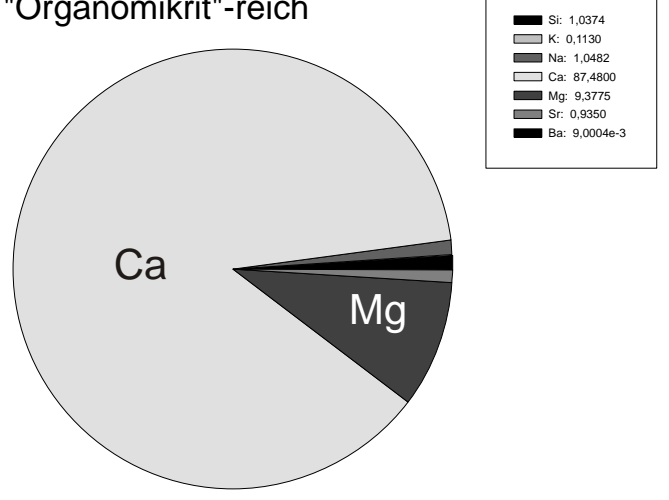

Shark Bay, SB 98/29

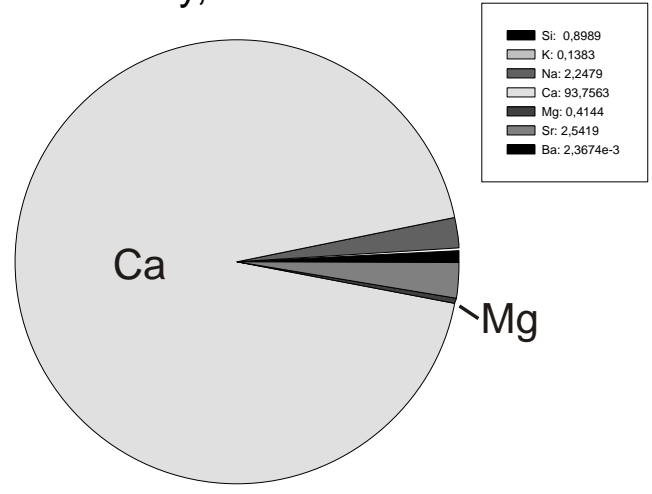

LWa 02/3

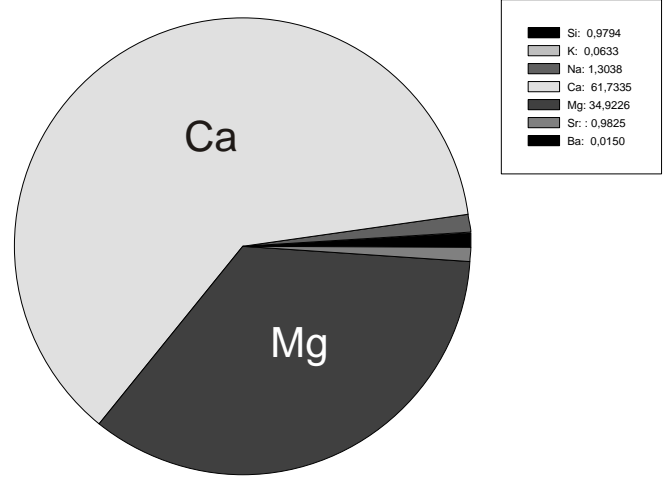




\section{Lebenslauf}

\section{Meike Caselmann}

geboren am 21.11.1974 in Braunschweig

Staatsangehörigkeit: deutsch

\section{Hochschulausbildung und wissenschaftlicher Werdegang}

$\begin{array}{ll}\text { 06/1994 } & \text { Abitur } \\ \text { 10/1994-03/1997 } & \begin{array}{l}\text { Studium der Geologie / Paläontologie an der Technischen Universität } \\ \text { Carolo-Wilhelmina zu Braunschweig }\end{array} \\ & \text { Vordiplom } \\ 01 / 1997 & \begin{array}{l}\text { Studium der Geologie-Paläontologie an der Georg-August-Universität } \\ \text { zu Göttingen }\end{array}\end{array}$

$08 / 2000$

Geologie-Diplom

10/2000-03/2003 Promotion am Geowissenschaftlichen Zentrum der Universität Göttingen, im Rahmen des von der DFG geförderten Forschungsprojektes

Re 665/18-1 und 18-2 (Projektleiter Prof. J. Reitner):

Biofilme, Makromoleküle und organische Restsubstanzen als Matrizen bei der Bildung von Organo- und Biomineralen - Geobiologische Faktoren bei der Evolution der Biomineralisation. 